\title{
Neuroinflammation as a cause of chronic pain
}

\author{
Citation for published version (APA):
}

Andrade, P. (2012). Neuroinflammation as a cause of chronic pain. [Doctoral Thesis, Maastricht University]. Datawyse / Universitaire Pers Maastricht. https://doi.org/10.26481/dis.20120920pa

Document status and date:

Published: 01/01/2012

DOI:

10.26481/dis.20120920pa

Document Version:

Publisher's PDF, also known as Version of record

\section{Please check the document version of this publication:}

- A submitted manuscript is the version of the article upon submission and before peer-review. There can be important differences between the submitted version and the official published version of record.

People interested in the research are advised to contact the author for the final version of the publication, or visit the DOI to the publisher's website.

- The final author version and the galley proof are versions of the publication after peer review.

- The final published version features the final layout of the paper including the volume, issue and page numbers.

Link to publication

\footnotetext{
General rights rights.

- You may freely distribute the URL identifying the publication in the public portal. please follow below link for the End User Agreement:

www.umlib.nl/taverne-license

Take down policy

If you believe that this document breaches copyright please contact us at:

repository@maastrichtuniversity.nl

providing details and we will investigate your claim.
}

Copyright and moral rights for the publications made accessible in the public portal are retained by the authors and/or other copyright owners and it is a condition of accessing publications that users recognise and abide by the legal requirements associated with these

- Users may download and print one copy of any publication from the public portal for the purpose of private study or research.

- You may not further distribute the material or use it for any profit-making activity or commercial gain

If the publication is distributed under the terms of Article $25 \mathrm{fa}$ of the Dutch Copyright Act, indicated by the "Taverne" license above, 


\title{
Neuroinflammation as a cause of chronic pain
}

\author{
Pablo Andrade
}


Copyright @ Pablo Andrade, Maastricht, 2012

Neuroinflammation as a cause of chronic pain

All rights reserved. No part of this book may be reproduced or transmitted in any form or by any means, without permission in writing, from the copyright holder.

Cover design: Rodrigo de los Santos. Image copyrights; Front image Sebastian Kaulitzki and Shuttersthock, back image Eliks and Shutterstock (downloaded with permission and reproduced with license).

Typesetting and layout: Pablo Andrade

ISBN 9789461591715 


\title{
Neuroinflammation as a
}

\section{cause of chronic pain}

\author{
DISSERTATION
}

\author{
To obtain the degree of \\ Doctor at Maastricht University, \\ on the authority of the Rector Magnificus, \\ Prof. dr. L.L.G. Soete \\ in accordance with the decision of the Board of Deans, \\ to be defended in public \\ on Thursday $20^{\text {th }}$ of September 2012 at 10.00 hrs.
}

by

Pablo Andrade

Born the $10^{\text {th }}$ of April of 1983 in Mexico City

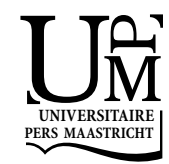




\section{Supervisors:}

Prof. Dr. H.W.M. Steinbusch

Prof. Dr. V. Visser-Vandewalle

\section{Co-supervisors:}

Dr. G. Hoogland

Dr. M.A. Daemen

\section{Assessment Committee:}

Prof. Dr. J.J. van Overbeeke (Chairman)

Prof. Dr. M.H. de Baets

Prof. Dr. L.W. van Rhijn

Prof. Dr. C. Sommer, University of Würzburg

Prof. Dr. W.C. Peul, University of Leiden 
A mis padres,

A mis hermanos,

À toi... 



\section{Contents}

\section{Chapter 1}

General Introduction

\section{Chapter 2}

Role of TNF-alpha during central sensitization in preclinical studies

\section{Chapter 3}

Inflammation and chronic pain: correlation between inflammatory profiles and pain evaluation in humans

\section{Chapter 4}

Tumor necrosis factor- $\alpha$ levels correlate with postoperative pain severity in lumbar disc hernia patients: opposite clinical effects between tumor necrosis factor receptor 1 and 2

\section{Chapter 5}

Elevated IL-1beta and IL-6 levels in lumbar herniated discs in patients with sciatic pain

\section{Chapter 6}

Elevated levels of TNF- $\alpha$ and TNFR1 in recurrent herniated lumbar discs correlate with chronicity of postoperative sciatic pain 
The thalidomide analgesic effect is associated with differential TNF- $\alpha$ receptor expression in the dorsal horn of the spinal cord as studied in a rat model of neuropathic pain

Chapter 8

TNF- $\alpha$ inhibitors alleviate experimentally-induced neuropathic pain by downregulation of TNF receptors

Chapter 9

Summary and conclusion

Acknowledgements

Curriculum Vitae

List of Publications 


\title{
Chapter 1
}

General introduction

\author{
Pablo Andrade
}




\section{Chapter 1}

\section{General introduction}

Neuropathic pain consists of a wide range of heterogeneous chronic conditions that are characterized by hyperalgesia (increased pain from a stimulus that normally provokes pain) and allodynia (pain due to a stimulus that does not normally provoke pain). According to the most recent classification by the International Association for the Study of Pain (IASP), it is defined as "pain caused by a lesion or disease of the somatosensory nervous system" (Merskey and Bogduk, 1994). This syndrome can originate from an injury or dysfunction in one or various segments of the peripheral and/or central nervous system, and its symptoms may vary depending on the extension, chronicity and nature of the insult (Baron, 2006). Physiologically, pain sensation results from the activation of nociceptive afferents indicating a warning for the damaged neural tissue. These concepts help to distinguish neuropathic from nociceptic types of pain, where the latter encodes noxious stimuli with a normally functioning somatosensory nervous system. In this respect, it is important to note that neuropathic pain is a clinical description that does not specify the lesion or disease, and that neuropathic pain is a condition composed from a variety of disorders (Jensen et al, 2011; Baron et al, 2010). Given these diverse conditions that give raise to neuropathic pain it is difficult to establish a single mechanism accountable for all clinical manifestations. Central research questions are therefore (1) how can nerve damage result in such a diversity of neuropathic pain intensity, and (2) which factors contribute to the chronicity of pain sensation.

One of the most common causes of chronic pain in adults is low back pain. About two out of three adults suffer from low back pain at some time during their life (Andersson, 1999). It represents the second most common cause for visits to a physician and for loss of working days, only after upper respiratory tract problems (Deyo and Weinstein, 2001). In this thesis we studied a subgroup of these patients, namely those that report an irradiation from pain into the buttock and leg. This presentation of radicular pain is also known as sciatica, a condition that normally arises from a constricted nerve root causing pain and numbness at the compression site and along the trajectory of the sciatic nerve. Most commonly, this condition is secondary to a lumbar disc herniation (LDH) that compresses the root at its exit from the spinal canal, and in fewer cases the spinal cord directly. In the majority of cases, sciatica can resolve spontaneously within a few weeks or with the use of conventional therapies, including physiotherapy, psychological support and pharmacotherapy. However, at one year follow-up, about one-third of the 
patients still experience high levels of pain and disability (Weber et al, 1993). For this group, surgery is considered an effective treatment to alleviate pain where symptoms persist for more than six to eight weeks (Valat et al, 2010). Yet, recent studies have shown that decompressive discectomy initially relieves leg pain but has unsatisfactory results at the long-term (Peul et al, 2007; Mariconda et al, 2006). So far, the underlying mechanisms of chronic sciatica following LDH surgery are still unclear. The two pain components observed in sciatica caused by LDH are neuropathic and nociceptive pain. Therefore, it has been mentioned as a mixed pain syndrome with a strong neuropathic pain component (Baron and Binder, 2004). This description is based on the following factors; (1) the local neuropathic damage originated by lesions of nociceptive sprouts inside the disc, (2) the mechanical neuropathic root pain caused by disc compression, and (3) the inflammatory neuropathic root pain caused by the degenerative disc. Biopsies collected in the course of a decompressive discectomy offer a unique source for studying potentially involved molecules.

For decades, it was believed that the mechanical compression created by the LDH was the sole cause of sciatica (Genevay et al, 2010). Recent preclinical studies further indicated the critical role of inflammation in the generation and maintenance of pain (George et al, 1999; Covery et al, 2002; Mulleman et al, 2006; Bianchi et al, 2007). Nowadays, the interaction between the immune and nervous systems are thought to be essential in the pathogenesis of chronic sciatica. The inflammatory process responsible for these changes is associated with neural hyperexcitability, vascular permeability, myelin changes and attraction of leukocytes (Kayama et al, 1998; Youn et al, 2008). The well documented proinflammatory, immunoregulatory and nociceptive properties of tumor necrosis factor- $\alpha$ (TNF- $\alpha$ ) make this key cytokine an attractive target to modulate pain (DeLeo et al, 2000; Xu et al, 2006; Sakuma et al, 2007). In this respect, animal models for neuropathic pain offer a unique opportunity to correlate pain behavior with spatially and temporally expressed cytokine pain. In this thesis we used the chronic constriction injury $(\mathrm{CCl})$ model in which the sciatic nerve is chronically injured by ligatures tied around the nerve. This model was chosen because it is characterized by a consistent and robust inflammatory process in the nervous system on the one hand, and by pain behavior as seen in a nerve root injury lesion model on the other hand.

The aim of this thesis was to assess the contribution of TNF- $\alpha$ and its receptors (TNFRs) to the severity of neuropathic pain. To address this question in a translational manner, we performed studies on both disc biopsies from LDH patients and spinal cord samples from the $\mathrm{CCl}$ model. First we investigated if 
sciatica in LDH patients correlated to spinal TNF- $\alpha$ and TNFR levels. To investigate if these inflammatory markers also contribute to the development of chronic pain, we compared levels from LDH patients that underwent a discectomy for the first time to those from patients that had had one or more discectomies. In the animal studies we focused on mechanisms of pain inhibition by inflammation blockage. Using the $\mathrm{CCl}$ rat model, we investigated the effect of anti-inflammatory drug treatment on the development of pain behavior and spinal TNF- $\alpha$ and TNFR expression.

Chapter 2 reviews the current understanding of the functional and molecular changes after peripheral nerve injury. Here, we focus on the role of TNF- $\alpha$ as crucial mediator in the development of neuropathic pain in animal models. This chapter also discusses possible implications of an unbalanced TNFR expression for pain behavior.

Clinical evidence for a correlation between inflammation and chronic pain is reviewed in chapter 3. Here, we outline the involvement of diverse inflammatory markers as underlying mechanism of pain signaling with regard to the clinical outcome and pain evaluation.

Chapter 4 describes our observations regarding the association between spinal TNF- $\alpha$ and TNFRs levels of LDH patients with the severity of leg pain after discectomy. Furthermore, we analyzed the connection between the differential expression of TNFR and the opposite clinical effects in pain sensation in these patients.

Using a similar study design, chapter 5 focuses on the expression pattern of spinal IL-1 $\beta$ and IL-6 levels in LDH patients related to preoperative pain and to the outcome of discectomy.

To analyze if TNF- $\alpha$ or TNFRs could play a role in the development of chronic pain in sciatica, we investigated their expression levels in spinal samples from $\mathrm{LDH}$ patients that underwent more than one decompressive discectomies. The predictive value of these data for a successful LDH surgery is discussed in chapter 6.

In the $\mathrm{CCl}$ model, we investigated if the analgesic effect induced by antiinflammatory drugs is accompanied by changes in spinal cord TNF- $\alpha$ and TNFR levels. As described in chapter 7 , we initially compared the effects of the selective TNF- $\alpha$ production inhibitor thalidomide with various anti-nociceptive drugs, including ZVAD (apoptosis inhibitor), IGF-1 (proliferation inductor) and NO-711 (GABA reuptake inhibitor). 
These experiments were further extended in chapter 8 by including TNF- $\alpha$ inhibitors etanercept and infliximab. In this chapter, we evaluated if TNFR expression in peripheral and central nervous system are associated with pain recovery. Because etanercept and infliximab have a different affinity for the two forms of TNF- $\alpha$, soluble TNF- $\alpha$ and membrane TNF- $\alpha$, we investigated how blockage of already circulating TNF- $\alpha$ affected the development of chronic neuropathic pain.

Finally, chapter 9 summarizes the findings from preceding chapters. These observations are briefly discussed and an overall conclusion of this thesis is made.

\section{References}

1. Andersson GBJ. Epidemiologic features of chronic low-back pain. Lancet 1999;354:581-5.

2. Baron R. Mechanisms of disease: neuropathic pain--a clinical perspective. Nat Clin Pract Neurol 2006;2:95-106.

3. Baron R, Binder A. [How neuropathic is sciatica? The mixed pain concept]. Orthopade 2004;33:568-75.

4. Baron R, et al. Neuropathic pain: diagnosis, pathophysiological mechanisms, and treatment. Lancet Neurol 2010;9:807-19.

5. Bianchi $M$, et al. Increased tumor necrosis factor-alpha and prostaglandin E2 concentrations in the cerebrospinal fluid of rats with inflammatory hyperalgesia: the effects of analgesic drugs. Anesth Analg 2007; 104:949-54.

6. Covey WC, et al. Expression of neuron-associated tumor necrosis factor alpha in the brain is increased during persistent pain. Reg Anesth Pain Med 2002;27:357-66.

7. DeLeo JA, et al. Transgenic expression of TNF by astrocytes increases mechanical allodynia in a mouse neuropathy model. Neuroreport 2000;11:599-602.

8. Deyo RA, Weinstein JN. Low Back Pain. N Engl J Med 2001;344:363370.

9. Genevay $S$, et al. Adalimumab in severe and acute sciatica: a multicenter, randomized, double-blind, placebo-controlled trial. Arthritis Rheum 2010;62:2339-46.

10. George A, et al. Serial determination of tumor necrosis factor-alpha content in rat sciatic nerve after chronic constriction injury. Exp Neurol 1999;160:124-32. 
11. Jensen TS, et al. A new definition of neuropathic pain. Pain 2011;152:2204-5.

12. Kayama $S$, et al. Cultured, autologous nucleus pulposus cells induce functional changes in spinal nerve roots. Spine 1998;23:2155-8.

13. Mariconda $M$, et al. Minimum 25-year outcome and functional assessment of lumbar discectomy. Spine 2006;31:2593-9.

14. Merskey B, Bogduk N. Classification of Chronic Pain Descriptions of Chronic Pain Syndromes and Definitions of Pain Terms. Second Edition. Seattle, 1994: IASP Press.

15. Mulleman D, et al. Pathophysiology of disk-related sciatica. I.--Evidence supporting a chemical component. Joint Bone Spine 2006;73:151-8.

16. Peul WC, et al. Surgery versus Prolonged Conservative Treatment for Sciatica. N Engl J Med 2007; 356:2245-2256.

17. Sakuma $Y$, et al. Up-regulation of p55 TNF alpha-receptor in dorsal root ganglia neurons following lumbar facet joint injury in rats. Eur Spine $\mathrm{J}$ 2007;16:1273-8.

18. Valat JP, et al. Sciatica. Best Pract Res Clin Rheumatol 2010;24:24152.

19. Weber $\mathrm{H}$, et al. The natural course of acute sciatica with nerve root symptoms in a double-blind placebo-controlled trial evaluating the effect of piroxicam. Spine 1993;18:1433-8.

20. Xu JT, et al. The role of tumor necrosis factor-alpha in the neuropathic pain induced by Lumbar 5 ventral root transection in rat. Pain 2006;123:306-21.

21. Youn $\mathrm{DH}$, et al. Exogenous tumor necrosis factor-alpha rapidly alters synaptic and sensory transmission in the adult rat spinal cord dorsal horn. J Neurosci Res 2008;86:2867-75. 


\section{Chapter}

Role of TNF-alpha during central sensitization in preclinical studies

Pablo Andrade, Veerle Visser-Vandewalle, Carolin Hoffmann, Harry W.M. Steinbusch, Marc A. Daemen, Govert Hoogland

Neurological Sciences (2011) 


\title{
Chapter 2
}

\begin{abstract}
Tumor necrosis factor-alpha (TNF- $\alpha$ ) is a principal mediator in pro-inflammatory processes that involve necrosis, apoptosis and proliferation. Experimental and clinical evidence demonstrate that peripheral nerve injury results in activation and morphological changes of microglial cells in the spinal cord. These adjustments occur in order to initiate an inflammatory cascade in response to the damage. Between the agents involved in this reaction, TNF- $\alpha$ is recognized as a key player in this process as it not only modulates lesion formation, but also because it is suggested to induce nociceptive signals. Nowadays, even though the function of TNF- $\alpha$ in inflammation and pain production seems to be generally accepted, diverse sources of literature point to different pathways and outcomes. In this review, we systematically searched and reviewed original articles from the past ten years on animal models of peripheral nervous injury describing TNF- $\alpha$ expression in neural tissue and pain behavior.
\end{abstract}




\section{Introduction}

Neuropathic pain (NP) is a chronic and extensive condition secondary to dysfunction or nerve damage, usually originated in the periphery. If the injurious stimulus perpetuates, dorsal horn neurons in the spinal cord become sensitized, enduring pain perception; process known as central sensitization (CS) [1-3]. Because inflammation is essential for NP development and that apoptosis is involved in CS, tumor necrosis factor-alpha (TNF- $\alpha$ ) becomes an interesting study target $[4,5]$. Several studies have investigated the relation between TNF- $\alpha$ expression in different nervous tissues and pain behavior; hitherto these studies have not been conclusive. Though in general, TNF- $\alpha$ is associated with cellular harm and pain behavior, it has also been shown that these effects are bound by temporal and spatial resolutions.

For decades, animal models have reproduced cardinal aspects of human pain, offering essential features for the preclinical study of NP. The main classification for NP models divides them in central pain, peripheral nerve injury, and secondary disease neuropathy models. In this review, we will focus on the peripheral nerve injury models in order to study the effect of these lesions on the process of central sensitization. The primary anatomical structures involved in these injury procedures are the sciatic nerve and dorsal root ganglion (DRG). These methods comprise partial ligature, complete ligature, root or nerve pinching, crushing, transection, and local inflammatory stimulus. The most representative examples include the chronic constriction injury model (Bennett model), partial sciatic nerve ligation model (Seltzer model), spinal nerve ligation, plexus avulsion, compression of the DRG/root, laminectomy, and disc injury [6-12].

TNF- $\alpha$ is considered to be one of the principal mediators in pro-inflammatory processes that involve necrosis, apoptosis and proliferation [5,13-15]. It is produced essentially by macrophages and T-lymphocytes in response to stressed or damaged tissue, and it may therefore serve as a systemic marker for tissue injury. For instance, complications found in inflammatory and autoimmune diseases are often accompanied by increased serum levels. In case of increased levels, a systemic inflammatory reaction leading to septic shock might occur. However, if TNF- $\alpha$ is present in low quantities it would generate a process responsible for local inflammatory symptoms, depending on the damaged structure [16-18]. In the nervous system, when a local peripheral injury is produced, it generates activation and morphological changes in dorsal horn microglia [19-23]. This process includes P2X4 receptors activation, 
followed by $\mathrm{Ca}^{2+}$ influx and p38 MAPK activation. As a consequence, microglia releases diffusible factors like TNF- $\alpha$, modifying neuronal excitability and thereby pain perception [24-26]. TNF- $\alpha$ has two distinct receptors, TNFreceptor-1 (TNFR1 or p55) and TNF-receptor-2 (TNFR2 or p75). TNFR1 main effect is related to apoptosis and is present in practically every cell; whereas TNFR2 has a limited constitutive expression and is basically associated with cell survival $[27,28]$. This is particularly important in pain attributable to different effects depending on specific receptor activation [29-31].

In this review, we present the functional and biochemical changes after peripheral nerve injury in animal models, focused on the role of TNF- $\alpha$ in the development of NP.

\section{Search Methods}

Articles were collected via PubMed from 1999 until July 2010; in order to analyze the understanding progress in the last decade, a period with increased standardized methods since the initial works associating TNF- $\alpha$ and neuropathic pain were performed. The following key-words and their combinations were used: chronic neuropathic pain, tumor necrosis factor, dorsal horn apoptosis, central sensitization, peripheral nerve injury, and neuropathic pain model. The obtained citations were examined by two independent reviewers in order to assure the application of the inclusion and exclusion criteria.

The inclusion criteria were: original full-text articles, preclinical reports, written in English, quantitative data on inflammatory response and pain production, mechanical compression or irritation to neural tissue, involvement of peripheral nervous system injury, and detailed measurement instrument with objective examination protocol. The exclusion criteria comprised: omission of TNF- $\alpha$ levels measurements, absence of standardized behavioral evaluations, presence of co-morbidity, use of TNF- $\alpha$ as inductor of lesion or inflammatory procedure, trauma related or spinal cord injury models, and involvement of other cytokines. 


\section{Results}

From the primary search a total of 2896 publications were identified. Based on titles and abstracts, 2814 articles were discarded due to irrelevance to the topic or duplication between different combinations. From the 82 prospective studies, 73 were selected according to the inclusion criteria. After applying the exclusion criteria we obtained 33 articles that were finally chosen for this review (Figure 1) [32-64].

From these 33 studies, 25 used rats of different strains and 8 applied genetically modified mice. The sample size of the experiments ranged from 7 to 360 animals, with a mean of 77 rodents per study. The most reproduced model was the ligation of the sciatic nerve denominated chronic constriction injury $(n=14)$, followed by nerve crushing $(n=8)$ and nerve transection $(n=4)$. The most common location to induce the inflammatory process was the sciatic nerve $(n=18)$ followed by the spinal nerves $(n=5)$.

Tissue samples were obtained from diverse structures, mostly the DRG $(n=16)$, sciatic nerve $(n=13)$ and spinal cord $(n=10)$ (Table 1; last section of the thesis). Laboratory techniques used for TNF- $\alpha$ determination in these biopsies included primarily immunohistochemistry $(n=22)$, and secondary ELISA $(n=8)$, qPCR $(n=7)$, and immunoblotting $(n=5)$. Follow-up of pain behavior monitoring ranged from 5 days to 12 weeks, being 14 days the most repeated period $(n=11)$. Not all of the included studies in this review applied behavioral testing related to allodynia and hyperalgesia $(n=11)$; the rest assessed mechanical sensitive thresholds primarily with von Frey filaments $(n=18)$, and thermal responsiveness with the Hargreaves test $(n=10)$. Some other conduct assessment techniques comprised the hot-plate $(n=2)$ and Randal-Selitto tests $(n=2)$.

Mechanical allodynia was assessed in 20 studies through application of pressure in affected and non-affected limbs in order to evaluate painful responses. Each one of these studies showed a significant decrease in all cases in nociceptive thresholds as a consequence of the injury procedure when compared to sham groups. Initiation of pain typically started 24 hours after the lesion intervention, reached maximum levels at 1 to 2 weeks, and returned close to presurgical control levels after 4 weeks. In two reports bilateral allodynia was present, in one case only for 7 days and referred in the second one as significant and long-lasting [42,64]. Nine articles evaluated pharmacotherapy and found the highest effectiveness when the drugs were 
Chapter 2

applied within 7 days after injury. In a specific case of cannabinoid administration, an antinociceptive effect was achieved after applying high doses from day 7 to 14 after injury $(20 \mathrm{mg} / \mathrm{kg}$ ) [36]. Opioid administration also reverted successfully the acute nociceptive effects, but only at increased doses and tolerance development (10 mg/kg b.i.d. for 5 days) [55].

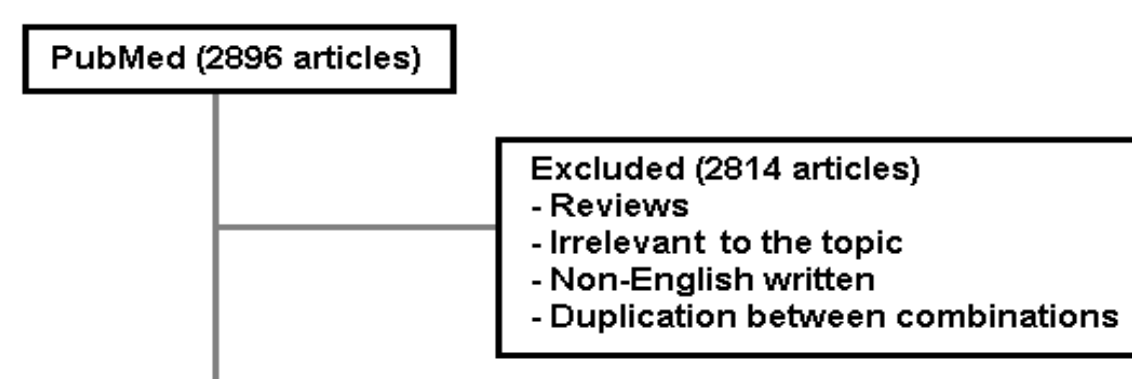

82 potentially relevant articles after selection from titles and abstracts

Excluded (9 articles)

- After inclusion criteria selection

Excluded (40 articles)

- After exclusion criteria application

- Non-related to TNF-alpha

- No molecular or behavioral tests

- Co-morbidity or parallel procedures

33 articles included for this review

References:

32-64

Figure1. Flow diagram representative of search strategy, identification, inclusion and exclusion of articles. 
Thermal hyperalgesia followed a similar pattern as mechanical allodynia, showing lower thresholds at short-term measurements, starting to decrease and even reaching baseline levels after 14 days. In one article TNFR1 deficient rodents did not exhibit significant painful behavior after heating stimulation at any time point after injury [63] (Table 2; last section of the thesis).

All models show increased TNF- $\alpha$ levels after injury. Pharmacological treatments that failed to decrease these levels included monotherapy of paracetamol, tramadol, cannabidiol, morphine, soluble TNF receptor, and IL-1 receptor antagonist. On the other hand, successful interventions that lower TNF- $\alpha$ expression included NMDA receptor antagonist MK-801, thalidomide, combined therapy of soluble TNF receptor with IL-1 receptor antagonist, chronic administration of morphine (dose-dependant with aggravation after interruption), a2-macrogobulin, nimesulide, a combination of paracetamol with tramadol, recombinant human erythropoietin and triamcinolone [32,34$36,43,45,55,61,62,64]$.

Chronologically, TNF- $\alpha$ expression was characterized by an immediate increase in affected structures starting as soon as one hour after injury $[45,56,59]$. Peak levels are detected around 24 hours, sustain for about three days, and in many cases then decreased, occasionally to baseline levels $[40,41,45,47]$. Some publications describe a lowering of TNF- $\alpha$ expression after this point, but the mainstream pointed out the maintenance of a plateau phase up to 1 to 2 weeks, followed by a periodical decline. Measurements performed at 3 and 4 weeks reveal weak signaling of the inflammatory process [37,39$41,45,47,54,56,59,60]$. Only one study documented prolonged increased TNF- $\alpha$ levels lasting at least 6 and 12 weeks, not only in nervous tissue but also in connective tissue, after applying the high force reaching task model [39].

The degree to which an altered TNFR1 and TNFR2 ratio contributes to pain behavior is still not clear. One study observed an increased TNFR1 expression several days after dorsal root lesion, while TNFR2 levels remained unchanged [44]. In contrast, a similar follow-up after sciatic nerve injury resulted in a 2-fold increased TNFR1 level and a 7-fold elevated TNFR2 level [41]. In yet another report showed that immediate measurement only after 6 hours post spinal nerve ligation, revealed that both receptors showed similar increased expression in the dorsal root ganglia and remained at comparable levels up to the maximum follow-up [59]. 


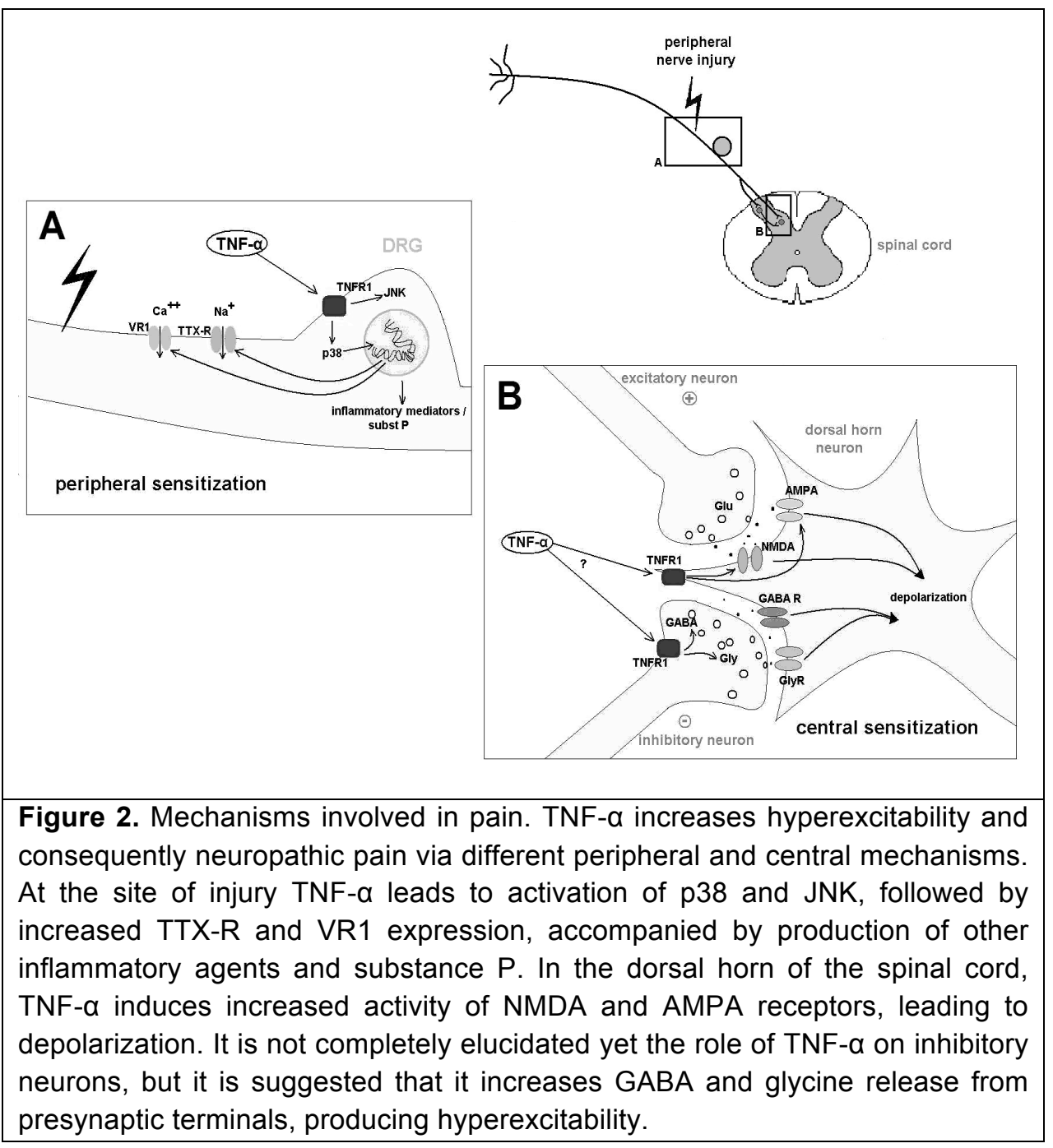

\section{Discussion}

In this review we have summarized the reports that appeared over the past 10 years and described peripheral injury-induced TNF- $\alpha$ expression in the nervous tissue and its role in the production of CS. To these days, there is a considerable amount of evidence to support the hypothesis that inflammatory process following tissue damage can lower pain thresholds. In this respect, many pathways and cytokines have been described to contribute in the 
development of chronic nociceptive signaling, including the three proinflammatory cytokines that induce activation of p38 MAPK: TNF- $\alpha$, IL-1 $\beta$ and IL-6 [25]. The reason to focus on TNF- $\alpha$ is because it is regarded as the archetypical proinflammatory agent, attributable to its function as an inductor of diverse processes that initiate and perpetuate the inflammatory response. At the same time, its increased expression in nervous tissues upon a pain stimulus, suggest that this cytokine is essential for the development of pain sensation [65-69].

Several authors published similar results in regard of TNF- $\alpha$ presence in peripheral and central nervous tissue after neural injury (Figure 2). However, part of the main divergences remain in the involvement and interactions of TNFR1 and TNFR2. Thus far, it seems that both receptor types play a different role in the development of allodynia and hyperalgesia depending on the site of the lesion and the moment of the cascade activation. For example, TNFR1 has shown constitutive expression in the uninjured spinal cord in modulatory centers like the dorsal horn, whereas these areas hardly express TNFR2 [44,70]. Nevertheless, this does not imply that after peripheral nerve injury and TNF- $\alpha$ release, TNFR2 could not be involved in nociceptive regulation [47]. This could also be dependant on the proximity of these lesions from the spinal cord, illustrated by earlier manifestation of pain in spinal nerve lesions (hours) than in sciatic nerve lesions (days) [45,59]. In judgment to the literature reported to our days, it is not still clear if the final nociceptive outcome is more dependant on the amount of cytokine or on the activation/inhibition of specific receptors (Figure 3).

The increase of TNF- $\alpha$ immunoreactive cells or mRNA levels showed a similar temporal resolution, regardless of the studied tissue or injury model. As previously described, TNF- $\alpha$ production typically starts to reach maximal levels within 24 hours, which are maintained for over 2 weeks and then usually drop $[37,39-41,45,47,54,56,59,60]$. This paradigm is important for understanding the development of painful symptomatology and for the therapeutic timing approach. Behavioral data show that the clinical presentation follows the presence of increased amounts of TNF- $\alpha$ in neural tissue [71-73]. Fluctuation in TNF-a levels tended to match with increased or decreased states of mechanical allodynia and thermal hyperalgesia. Progressive nociception in injured animals was established after the first days and maintained until its highest point around the second week, followed by a graduate drop up to baseline thresholds $[37,54,56]$. This transient pain behavior may be a consequence of the continuous adaptation of pain perception that familiarizes the animal with the 
applied stimuli, yet in each study the cytokine levels correlated closely to the pain sensation $[41,53]$. In this respect, TNF- $\alpha$ involvement in pain could also be inferred in studies where wild-type mice showed greater levels of pain when compared to genetically TNF knock-out animals $[74,75]$.

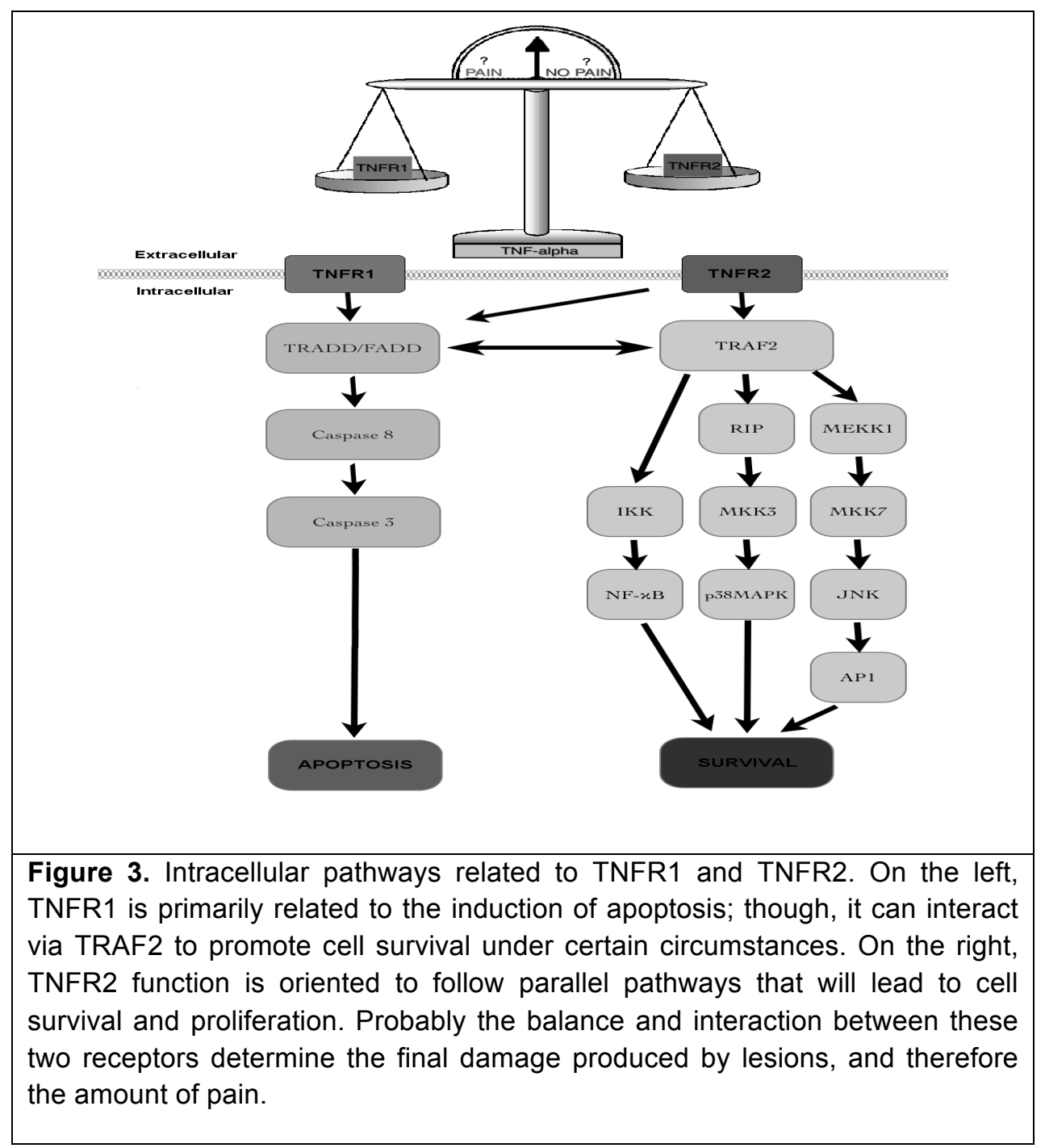

The pharmacological therapies proved to be successful when applied during the first 7 days post lesion, which is consistent with the temporal resolution of TNF- 
a expression, probably avoiding the initial inflammatory response [66,76]. This is also an essential matter in the apparent dual intervention of TNF- $\alpha$ in neurons as toxic or protective agent. Originally, the function of this cytokine has been described as harmful, but previous evidence and data in this review suggest that protection and regeneration could be involved in a receptor-dependant mode. It has been described that TNFR2 has a fundamental participation in remyelination of neurons after injury, probably linked to its late participation in the inflammatory cascade $[44,70,77,78,79]$. This suggestion also comes from delayed expression of TNFR2, which coincides with behavioral recovery in the late phases of pain sensation. Thus, it is of vital importance to understand the temporal expression of TNF- $\alpha$ and its receptors in order to develop a successful therapeutic strategy. To these days, we can observe that the same cytokine at different moments, acting on different receptors, could bring opposite histological and clinical outcomes. These observations bring the query of which factors are more determinant for the production of pain, the concentration of TNF- $\alpha$ or the TNFR1 - TNFR2 expression ratio. In this respect, a key question that remains is why patients with chronic NP do not benefit from this self-limiting protective mechanism. We believe that a balance alteration between the TNF- $\alpha$ receptors could determine the severity of pain, not only in a temporal expression (acute or chronic) but also on the extension of the produced damage. Animal models have proven to develop and reproduce pain in a comparable manner to human observations. In summary, there is evidence from physical injury models, transgenic models, and pharmacological interference studies that TNF- $\alpha$ expression in the spinal cord modulates pain sensation. However, future perspectives for treatment still require better understanding of its receptors and intracellular pathways interactions as pain modulators. Therefore, further translational studies may be performed to address this challenging topic and design more effective therapies by regulating inflammatory-induced pain sensation.

\section{References}

1. Gracely $\mathrm{RH}$, et al. Painful neuropathy: altered central processing maintained dynamically by peripheral input. Pain 1992;51:175-194.

2. Woolf CJ, King AE. Dynamic alterations in the cutaneous mechanoreceptive fields of dorsal horn neurons in the rat spinal cord. $\mathrm{J}$ Neurosci 1990;10:2717-2726. 
3. Woolf CJ, Wall PD. The relative effectiveness of $C$ primary afferent fibres of different origins in evoking a prolonged facilitation of the flexor reflex in the rat. J Neurosci 1986;6:1433-1443.

4. Ma QP, Woolf CJ. Progressive tactile hypersensitivity: An inflammationinduced incremental increase in the excitability of the spinal cord. Pain 1996;67:97-106.

5. Morgan MJ, et al. TNFalpha and reactive oxygen species in necrotic cell death. Cell Res 2008;18:343-9.

6. Bennett GJ, Xie YK. A peripheral mononeuropathy in rat that produces disorders of pain sensation like those seen in man. Pain 1988;33:87107.

7. Kim SH, Chung JM. An experimental model for peripheral neuropathy produced by segmental spinal nerve ligation in the rat. Pain 1992;50:355-63.

8. Massie JB, et al. A preclinical post laminectomy rat model mimics the human post laminectomy syndrome. J Neurosci Methods 2004;137:283-9.

9. Olmarker K, et al. Pathogenesis of sciatic pain: a study of spontaneous behavior in rats exposed to experimental disc herniation. Spine 2002;27:1312-7.

10. Rodrigues-Filho $R$, et al. Pharmacological characterisation of the rat brachial plexus avulsion model of neuropathic pain. Brain Res 2004;1018:159-70.

11. Seltzer Z, et al. A novel behavioral model of neuropathic pain disorders produced in rats by partial sciatic nerve injury. Pain 1990;43:205-18.

12. Song $X J$, et al. Mechanical and thermal hyperalgesia and ectopic neuronal discharge after chronic compression of dorsal root ganglia. $J$ Neurophysiol 1999;82:3347-58.

13. Cope A, et al. The role of TNF-alpha and related cytokines in the development and function of the autoreactive T-cell repertoire. Res Immunol 1997;148:307-312.

14. Kim YS, et al. TNF-induced activation of the Nox1 NADPH oxidase and its role in the induction of necrotic cell death. Mol Cell 2007;26:675-87.

15. Sakon S, et al. NF-kappaB inhibits TNF-induced accumulation of ROS that mediate prolonged MAPK activation and necrotic cell death. EMBO J 2003;22:3898-3909.

16. Lee SC, et al. Cytokine production by human fetal microglia and astrocytes. Differential induction by lipopolysaccharide and IL-1 beta. J Immunol 1993;150:2659-2667. 
17. McCoy MK, Tansey MG. TNF signaling inhibition in the CNS: implications for normal brain function and neurodegenerative disease. $\mathrm{J}$ Neuroinflammation 2008;5:45.

18. Sawada M, et al. Production of tumor necrosis factor-alpha by microglia and astrocytes in culture. Brain Res 1989;491:394-397.

19. Inoue K. The function of microglia through purinergic receptors: neuropathic pain and cytokine release. Pharmacol Ther 2006;109:21026.

20. Kreutzberg GW. Microglia: a sensor for pathological events in the CNS. Trends Neurosci 1996;19:312-318.

21. Liu B, Hong JS. Role of microglia in inflammation-mediated neurodegenerative diseases: mechanisms and strategies for therapeutic intervention. J Pharmacol Exp Ther 2003; 304:1-7.

22. Si Q, et al. 15-deoxy-Delta12,14-prostaglandin J2 inhibits IFN-inducible protein $10 / C X C$ chemokine ligand 10 expression in human microglia: mechanisms and implications. J Immunol 2004;173:3504-13.

23. Stoll G, Jander $S$. The role of microglia and macrophages in the pathophysiology of the CNS. Prog Neurobiol 1999;58:233-247.

24. Hide $I$, et al. Extracellular ATP triggers tumor necrosis factor-alpha release from rat microglia. J Neurochem 2000;75: 965- 972.

25. Inoue K, Tsuda M. Microglia and neuropathic pain. Glia 2009;57:146979.

26. Suzuki T, et al. Production and release of neuroprotective tumor necrosis factor by P2X7 receptor-activated microglia. J Neurosci 2004;24:1-7.

27. Carpentier I, et al. Function and regulation of tumor necrosis factor type 2. Curr Med Chem 2004;11:2205-12.

28. Faustman D, Davis M. TNF receptor 2 pathway: drug target for autoimmune diseases. Nat Rev Drug Discov 2010;9:482-93.

29. Chen G, Goeddel DV. TNF-R1 signaling: a beautiful pathway. Science 2002;296:1634-1635.

30. Lee KM, et al. Tumor necrosis factor receptor 1 induces interleukin-6 upregulation through NF-kappaB in a rat neuropathic pain model. Eur $\mathrm{J}$ Pain 2009;13:794-806.

31. MacEwan DJ. TNF receptor subtype signalling: differences and cellular consequences. Cell Signal 2002;14:477-92.

32. Arandjelovic $S$, et al. A derivative of the plasma protease inhibitor alpha(2)-macroglobulin regulates the response to peripheral nerve injury. J Neurochem 2007;103:694-705. 
33. Bianchi $M$, et al. Increased substance $P$ and tumor necrosis factoralpha level in the paws following formalin injection in rat tail. Brain Res 2004;1019:255-8.

34. Bianchi $M$, et al. Increased tumor necrosis factor-alpha and prostaglandin E2 concentrations in the cerebrospinal fluid of rats with inflammatory hyperalgesia: the effects of analgesic drugs. Anesth Analg 2007;104:949-54.

35. Campana WM, et al. Erythropoietin reduces Schwann cell TNF-alpha, Wallerian degeneration and pain-related behaviors after peripheral nerve injury. Eur J Neurosci 2006;23:617-26.

36. Costa B, et al. The non-psychoactive cannabis constituent cannabidiol is an orally effective therapeutic agent in rat chronic inflammatory and neuropathic pain. Eur J Pharmacol 2007;556:75-83.

37. Covey WC, et al. Expression of neuron-associated tumor necrosis factor alpha in the brain is increased during persistent pain. Reg Anesth Pain Med 2002;27:357-66.

38. Dubový $P$, et al. Intra- and extraneuronal changes of immunofluorescence staining for TNF-alpha and TNFR1 in the dorsal root ganglia of rat peripheral neuropathic pain models. Cell Mol Neurobiol 2006;26:1205-17.

39. Elliott $M B$, et al. High force reaching task induces widespread inflammation, increased spinal cord neurochemicals and neuropathic pain. Neuroscience 2009;158:922-31.

40. George A, et al. Serial determination of tumor necrosis factor-alpha content in rat sciatic nerve after chronic constriction injury. Exp Neurol 1999;160:124-32.

41. George A, et al. Tumor necrosis factor receptor 1 and 2 proteins are differentially regulated during Wallerian degeneration of mouse sciatic nerve. Exp Neurol 2005;192:163-6.

42. Hatashita $\mathrm{S}$, et al. Contralateral neuropathic pain and neuropathology in dorsal root ganglion and spinal cord following hemilateral nerve injury in rats. Spine 2008;33:1344-51.

43. Hayashi R, et al. Systemic glucocorticoid therapy reduces pain and the number of endoneurial tumor necrosis factor-alpha (TNFalpha)-positive mast cells in rats with a painful peripheral neuropathy. J Pharmacol Sci 2008;106:559-65.

44. Holmes GM, et al. Immunocytochemical localization of TNF type 1 and type 2 receptors in the rat spinal cord. Brain Res 2004;1025:210-9. 
45. Kato $\mathrm{K}$, et al. Distribution and tumor necrosis factor-alpha isoform binding specificity of locally administered etanercept into injured and uninjured rat sciatic nerve. Neuroscience 2009;160:492-500.

46. Kleinschnitz $C$, et al. Contralateral cytokine gene induction after peripheral nerve lesions: dependence on the mode of injury and NMDA receptor signaling. Mol Brain Res 2005;136:23-8.

47. Lee HL, et al. Temporal expression of cytokines and their receptors mRNAs in a neuropathic pain model. Neuroreport 2004;15:2807-11.

48. Li X, et al. Tumor necrosis factor-alpha of Red nucleus involved in the development of neuropathic allodynia. Brain Res Bull 2008; 77:233236.

49. Murata $Y$, et al. Distribution and appearance of tumor necrosis factoralpha in the dorsal root ganglion exposed to experimental disc herniation in rats. Spine 2004;29:2235-41.

50. Murata $Y$, et al. Selective inhibition of tumor necrosis factor-alpha prevents nucleus pulposus-induced histologic changes in the dorsal root ganglion. Spine 2004;29:2477-84.

51. Murata $Y$, et al. Nucleus pulposus-induced apoptosis in dorsal root ganglion following experimental disc herniation in rats. Spine 2006;31:382-90.

52. Ohtori S, et al. TNF-alpha and TNF-alpha receptor type 1 upregulation in glia and neurons after peripheral nerve injury: studies in murine DRG and spinal cord. Spine 2004;29:1082-8.

53. Okamoto $\mathrm{K}$, et al. Pro- and anti-inflammatory cytokine gene expression in rat sciatic nerve chronic constriction injury model of neuropathic pain. Exp Neurol 2001;169:386-91.

54. Otoshi K, et al. The reactions of glial cells and endoneurial macrophages in the dorsal root ganglion and their contribution to painrelated behavior after application of nucleus pulposus onto the nerve root in rats. Spine 2010;35:264-71.

55. Raghavendra $V$, et al. The role of spinal neuroimmune activation in morphine tolerance/hyperalgesia in neuropathic and sham-operated rats. J Neurosci 2002;22:9980-9.

56. Sacerdote $P$, et al. Transient early expression of TNF-alpha in sciatic nerve and dorsal root ganglia in a mouse model of painful peripheral neuropathy. Neurosci Lett 2008;436:210-3.

57. Sakuma Y, et al. Up-regulation of p55 TNF alpha-receptor in dorsal root ganglia neurons following lumbar facet joint injury in rats. Eur Spine $\mathrm{J}$ 2007;16:1273-8. 
58. Schäfers $M$, et al. Combined epineurial therapy with neutralizing antibodies to tumor necrosis factor-alpha and interleukin-1 receptor has an additive effect in reducing neuropathic pain in mice. Neurosci Lett 2001;310:113-6.

59. Schäfers $M$, et al. Spinal nerve ligation induces transient upregulation of tumor necrosis factor receptors 1 and 2 in injured and adjacent uninjured dorsal root ganglia in the rat. Neurosci Lett 2003;347:179-82.

60. Sekiguchi $M$, et al. Comparison of neuropathic pain and neuronal apoptosis following nerve root or spinal nerve compression. Eur Spine J 2009;18:1978-85.

61. Sweitzer S, et al. Intrathecal interleukin-1 receptor antagonist in combination with soluble tumor necrosis factor receptor exhibits an antiallodynic action in a rat model of neuropathic pain. Neuroscience 2001;103:529-39.

62. Uçeyler N, et al. Early cytokine gene expression in mouse CNS after peripheral nerve lesion. Neurosci Lett 2008;436:259-64.

63. Vogel $\mathrm{C}$, et al. Altered pain behavior and regeneration after nerve injury in TNF receptor deficient mice. J Peripher Nerv Syst 2006;11:294-303.

64. Xu JT, et al. The role of tumor necrosis factor-alpha in the neuropathic pain induced by Lumbar 5 ventral root transection in rat. Pain 2006;123:306-21.

65. Homma $Y$, et al. A comparison of chronic pain behavior following local application of tumor necrosis factor alpha to the normal and mechanically compressed lumbar ganglia in the rat. Pain 2002;95:23946.

66. Marchand $F$, et al. Effects of Etanercept and Minocycline in a rat model of spinal cord injury. Eur J Pain 2009;13:673-81.

67. Murata $\mathrm{Y}$, et al. Changes in pain behavior and histologic changes caused by application of tumor necrosis factor-alpha to the dorsal root ganglion in rats. Spine 2006;31:530-5.

68. Ozaktay AC, et al. Effects of interleukin-1 beta, interleukin-6, and tumor necrosis factor on sensitivity of dorsal root ganglion and peripheral receptive fields in rats. Eur Spine J 2006;15:1529-37.

69. Peng $X M$, et al. Tumor necrosis factor-alpha contributes to below-level neuropathic pain after spinal cord injury. Ann Neurol 2006;59:843-51.

70. Hermann GE, et al. TNF alpha-p55 receptors: medullary brainstem immunocytochemical localization in normal and vagus nerve-transected rats. Brain Res 2004;1004:156-66. 
71. Kawasaki $\mathrm{Y}$, et al. Cytokine mechanisms of central sensitization: distinct and overlapping role of interleukin-1beta, interleukin-6, and tumor necrosis factor-alpha in regulating synaptic and neuronal activity in the superficial spinal cord. J Neurosci 2008;28:5189-94.

72. Schäfers $M$, et al. Selective stimulation of either tumor necrosis factor receptor differentially induces pain behavior in vivo and ectopic activity in sensory neurons in vitro. Neuroscience 2008;157:414-23.

73. Youn $\mathrm{DH}$, et al. Exogenous tumor necrosis factor-alpha rapidly alters synaptic and sensory transmission in the adult rat spinal cord dorsal horn. J Neurosci Res 2008;86:2867-75.

74. DeLeo JA, et al. Transgenic expression of TNF by astrocytes increases mechanical allodynia in a mouse neuropathy model. Neuroreport 2000;11:599-602.

75. Yamashita $M$, et al. Tumor necrosis factor-alpha in the nucleus pulposus mediates radicular pain, but not increase of inflammatory peptide, associated with nerve damage in mice. Spine 2008;33:183642.

76. Zanella JM, et al. Effect of etanercept, a tumor necrosis factor-alpha inhibitor, on neuropathic pain in the rat chronic constriction injury model. Spine 2008;33:227-34.

77. Arnett HA, et al. TNF alpha promotes proliferation of oligodendrocyte progenitors and remyelination. Nat Neurosci 2001;4:1116-22.

78. Fontaine $\mathrm{V}$, et al. Neurodegenerative and neuroprotective effects of tumor Necrosis factor (TNF) in retinal ischemia: opposite roles of TNF receptor 1 and TNF receptor 2. J Neurosci 2002;22:RC216.

79. Aggarwal BB. Signalling pathways of the TNF superfamily: a doubleedged sword. Nat Rev Immunol 2003;3:745-56. 



\section{Chapter}

\section{Inflammation and chronic pain: correlation between inflammatory profiles and pain evaluation in humans}

Pablo Andrade, Veerle Visser-Vandewalle, Mathias Leinders, Ronald Deumens, Marc A. Daemen, Harry W.M. Steinbusch, Wim A. Buurman, Govert Hoogland 


\section{Chapter 3}

\section{Abstract}

Chronic pain (CP) is a widespread disabling condition that affects almost one fifth of the world population, posing a great economic burden on society in terms of working days lost and treatment costs. To date, the underlying mechanisms of CP are not yet fully understood. Several lines of evidence have suggested that inflammation and its associated damage may play a critical role in the initiation and maintenance of pain. However, most of this extensive knowledge comes from preclinical studies, where the translational application of these insights may not always be possible on the human scenario. The first reports examining the direct association of inflammatory cytokines and painrelated symptomatology in humans appeared only in recent years. We aim to provide a complete overview of the role of inflammatory cytokines on CP in humans, and the correlation between these agents and its consequences for pain assessment methods used in clinical practice. The most representative preclinical findings on inflammation and nociception relevant to the human condition are discussed in this review. We also examine the most novel human imaging studies used to locate inflammatory processes responsible for $\mathrm{CP}$ and the drugs employed to modulate these responses. It is clear that further translational research is needed in order to elucidate the pathophysiology of pain in humans. Ultimately, these insights could lead us to perform earlier diagnoses and develop more effective medications. 


\section{Introduction}

Establishing a complete and concrete definition for chronic or persistent pain is a complicated task. According to the International Association for the Study of Pain (IASP), it is described as "that pain that persists even when the healing process has occurred" (IASP, 1986), being often considered, for practical purposes, as a period of three months or more with pain (IASP, 1986; Smith et al., 1999). However, in reality, many of the disorders responsible for persistent pain escape from this temporal classification. According to their pathophysiology, chronic pain disorders can be divided in peripheral or central mechanisms, caused by hyperexcitability of nociceptors or malfunction or injury of the nervous system. In both cases, inflammation (and its associated damage) is considered as one of the underlying mechanisms for persistent pain (Baron, 2006; Basbaum et al., 2009). The proalgesic effects produced by inflammatory cytokines on nociceptors have been well documented in several animal and in vitro studies (Sweitzer et al., 2001; Vogel et al., 2006; Wagner and Myers, 1996; Watkins et al., 1995). The cascade of events unleashed by these cytokines may result in hyperalgesic effects from other mediators like neuropeptides, bradykinin, prostaglandins and nerve growth factor (for review see Üçeyler et al., 2009). It was not until the last decade that the first studies in humans correlating the intensity of pain with inflammatory cytokines were performed. In general, these attempts to translate conclusions obtained from preclinical studies into the human condition have corroborated the previous findings. However, the diversity of pain disorders and human tissue samples analyzed might complicate the understanding of the effects that inflammatory cytokines exert on the human body with regard to pain.

We reviewed all articles in which a correlation was described between inflammatory cytokines and the clinical evaluation of pain in humans. The reports were found via Medline search (PubMed) up to January 2012 using the following keywords and their combinations: cytokines, neuropathy, pain, neuropathic pain, patient, human, inflammatory cytokines, biopsy, blood, cerebrospinal fluid (CSF), painful neuropathy, symptomatology, visual analogue scale, VAS, and chronic pain. In total, 253 articles were found in the initial search. Ultimately, after citation tracking and exclusion of articles due to overlap or lack of clinical data, 35 reports were selected for cytokine determination in human tissue biopsies in this review. Subsequently, pertinent references obtained from preclinical studies and imaging techniques were added to this report in order to describe the contemporary understanding about inflammation and pain. Since these preclinical and imaging reports were not part of the main 


\section{Chapter 3}

objective of this study, their inclusion into the review was not systematically performed.

This review aims at providing an overview of inflammation as an underlying mechanism in pain signaling with regard to the clinical outcome and pain evaluation in humans; likewise, we suggest future directions for research and for better health care for patients.

\section{Inflammation and pain in preclinical studies}

To date, the majority of knowledge that we have on the pathophysiology of pain comes from preclinical studies. However, the correlation of these findings to the human condition is limited mainly due to the difficulty to evaluate spontaneous pain behavior in animals, and because of the differences between the time of initiation and maintenance of pain in animals and humans. Although inflammatory cytokines maintain a similar temporal curve among species, with respect to persistent pain, animal models still differ from the clinical situation because of their different chronic outcome (Baron et al., 2010).

\subsection{Animal models}

During decades numerous models have been employed to reproduce pain behavior in animals, mainly in rodents. Multiple anatomical segments from the peripheral nervous system have been used to modulate the pain-generating network. Essentially, the targets involved include the peripheral nerves (common peroneal, tibial and sural nerves), sciatic nerve, dorsal root ganglion (DRG), spinal nerves, and dorsal roots (for review see Sorkin and Yaksh, 2009). These structures have been manipulated in order to exert mechanical allodynia and thermal hyperalgesia, mainly via intraplantar injections of inflammatory agents, spared nerve injury, chronic constriction injury $(\mathrm{CCl})$ (sciatic nerve loose ligation), spinal nerve ligation, DRG compression, spinal nerve ligation, and dorsal root crush or compression (Bennett and Xie, 1988; George et al., 2005; Kim and Chung, 1992; Larson et al., 1986; Schäfers et al., 2003b; Seltzer et al., 1990; Song et al., 1999). The findings from these models show increased expression of markers such as TNF- $\alpha$, IL-1 $\beta$, IL-6, IL-10, Iba1, OX-42, GFAP, among others, in association with reduced pain thresholds (Andrade et al., 2011a; Berger et al., 2011; Lee et al., 2004; Sakuma et al., 
2007; Üçeyler and Sommer, 2008; Winkelstein and DeLeo, 2002). These observations indicate that after a peripheral neuronal injury, inflammation is responsible for the instatement and continuation of pain behavior in animals. Furthermore, in vivo studies show mitigation of pain after anti-inflammatory drug administration or knocking-out neuroimmune-related receptors (Verri et al., 2006; Vogel et al., 2006; Zhang et al., 2011). Finally, solid evidence about this correlation comes from studies where animals developed robust pain behavior after proinflammatory cytokines were administered (Oka et al., 1993; Ozaktay et al., 2006; Schäfers et al., 2003a; Schäfers et al., 2003c; Zhang et al., 2011). It is important to remark that the effects on pain generated by these cytokines have proven to be primarily related to the induction of proalgesic agents and the increase of the inflammatory process, and secondarily to the direct action on neuronal receptors.

\subsection{In vitro studies}

Long-term potentiation (LTP) has been largely described as a mechanism responsible for increased excitability in the dorsal horn of the spinal cord, principally documented in unmyelinated C-fibers afferents (for review see ( $\mathrm{Ji}$ et al., 2003)). Indeed, these LTP C-fiber evoked potentials can be caused by inflammatory mediators after nerve injury by altering the excitability at the terminal-membrane (Gold et al., 1998; Ji et al., 2002; Liu et al., 2007). Under normal basal conditions inhibitory interneurons reduce nociceptive output, thereby diminishing pain sensation. However, after peripheral nerve damage, invading immune cells, activated microglia, neurons and astrocytes are known to be involved in the production of proinflammatory mediators, such as PGE2, cytokines, chemoattractants, NO and neurotrophic factors (BDNF, NGF). PGE2 for example is known to increase intracellular concentrations of CAMP that activates PKA, which in turn can phosphorylate and thereby functionally inhibit glycine receptors that contain the alpha3 subunit. In addition, normally silent NMDA glutamate receptors in the postsynaptic neuron facilitate signaling and as a consequence of this transmission, the increased excitability that exits the output neuron will eventually alter pain processing. Moreover, BDNF is known to downregulate $\mathrm{KCC} 2$ transporters, which ultimately leads to diminished inhibitory neurotransmission (Zeilhofer et al., 2012) (see Figure 1).

Electrophysiological studies show that cytokines can modulate neuronal excitability through several receptors and ion channels (for review see 


\section{Chapter 3}

(Schäfers and Sorkin, 2008)). Earlier studies describe cytokines' direct action on sensory neurons after a proinflammatory agent is injected. Nociceptors increase their susceptibility for noxious heat and to stimulation with cold, heat and touch as a response to the cytokine administration (Fukuoka et al., 1994; Obreja et al., 2002). Recent studies have shown that direct injection of cytokines to the spinal cord and the nerve root increases the frequency of spontaneous discharges of wide dynamic range neurons, but not amplitude, and also enhances the response of these neurons to noxious stimuli (Onda et al., 2002; Zhang et al., 2011).

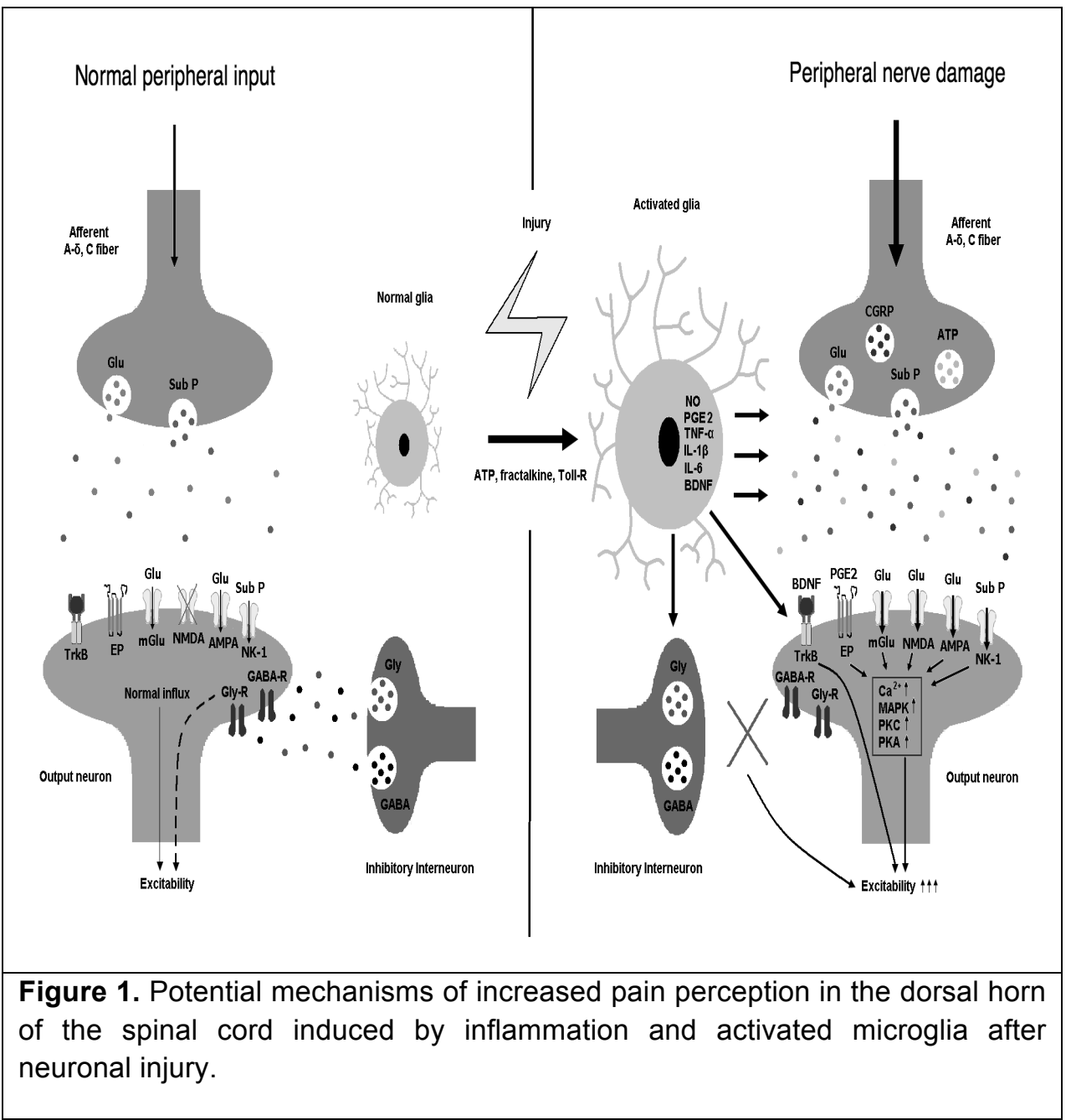




\section{Effects of inflammatory mediators on pain}

\subsection{Proinflammatory cytokines}

\subsubsection{Tumor necrosis factor- $\alpha$}

TNF- $\alpha$ is involved in the innate immunity and it is described as one of the primary agents of the acute inflammatory response against infections and tumors. TNF- $\alpha$ is a proinflammatory cytokine secreted mainly by macrophages, although mast cells, microglia and $T$ cells are also known to produce this protein. This cytokine can be expressed as a membrane bound precursor (mTNF- $\alpha ; 26 \mathrm{kD}$ ), capable to exert its effects in a cell-to-cell approach (Zhou et al., 2010). In order to be secreted as the mature TNF- $\alpha$ soluble form (sTNF- $\alpha$ ), the membrane molecule needs to be cleaved by the metalloproteinase called TNF- $\alpha$ converting enzyme (TACE). Once the sTNF- $\alpha$ is secreted, the protein can be found as a $17 \mathrm{kD}$ polypeptide or in a polymerized form of $51 \mathrm{kD}$. TNF- $\alpha$ receptors are part of a large family of receptors that contain rich cysteine domains outside the cell. TNF- $\alpha$ exerts its functions via two distinct receptors, the constitutive TNF- $\alpha$ receptor 1 (TNFR1) or the inducible TNF- $\alpha$ receptor 2 (TNFR2). The final effect and function of the former is associated to apoptosis, whereas the latter is related to proliferation and cell survival (Chen and Goeddel, 2002; Faustman and Davis, 2010).

The participation of TNF- $\alpha$ in chronic pain is mediated via different peripheral and central mechanisms. The main actions of TNF- $\alpha$ in the periphery come as a response to nerve injury or direct stimulation of neuronal tissue. Spinal nerve crush and loose ligation of the sciatic nerve in rats and mice have been shown to produce a decrease in the mechanical and thermal withdrawal thresholds, related in both cases to the early upregulation of TNF- $\alpha$ (George et al., 2005; Hatashita et al., 2008; Sacerdote et al., 2008). Similar behavioral findings were obtained after direct intraneural injection of recombinant TNF- $\alpha$ in rats (Sorkin and Doom, 2000). Furthermore, the amount of TNF- $\alpha$ injected responsible for the pain behavior was similar to the quantity of cytokine measured in injured animals (Zelenka et al., 2005). Similarly, exogenous application of TNF- $\alpha$ after nerve ligation induces increased sensitivity on neurons of injured and contiguous naïve nerves (Schäfers et al., 2003a). The central mechanisms of TNF- $\alpha$ are primarily mediated through dorsal horn neurons and glial cells. In a similar manner than peripheral nerves, exogenous administration of TNF- $\alpha$ in the spinal cord (intrathecal injection) elicits pain (allodynia/hyperalgesia) and results in altered synaptic transmission with increased frequency rates (Youn et 


\section{Chapter 3}

al., 2008; Zhang et al., 2011). Moreover, if the receptors are selectively activated or knocked-out, TNFR1 has been shown to play a crucial role on neuronal excitation and pain behavior induction; TNFR2 on the contrary has been shown to have a limited contribution in these areas, and are apparently dependent on TNFR1 action or tissue injury (Schäfers et al., 2008; Zhang et al., 2011). Several lines of evidence also suggest that TNF- $\alpha$ modifies the activity of a variety of ion channels such as calcium, potassium and sodium channels (for review see Czeschik et al., 2008). Besides the neuronal tissue, spinal cord glia has been largely implicated as well in chronic pain states. Increased activity of microglia and astrocytes has been associated with exaggerated pain responses (Watkins et al., 2001a; b). In studies where an envelope glycoprotein (gp120) was injected intrathecally (Milligan et al., 2001) or in the sciatic nerve (Zheng et al., 2011) in rats, the immune stimulation elicited mechanical allodynia was elicited and with a manifest colocalization of TNF- $\alpha$ in the spinal cord with markers for microglia (Iba1) and astrocytes (GFAP), suggesting that the cytokine is released from the activated glia. In respect of its interaction with other mediators, TNF- $\alpha$ can synergize and activate other cytokines such as IL$1 \beta$, IL-6 and IL-8 (Leung and Cahill, 2010); in addition, it has been shown that the hyperalgesic effects of other cytokines (e.g. IL-17) are dependent on TNFmediated neutrophil infiltration (McNamee et al., 2011).

\subsubsection{Interleukin-1 $\beta$}

$\mathrm{IL}-1 \beta$ is a prototypic proinflammatory cytokine that belongs to the IL-1 family, which is also composed by the interleukin-1 receptor antagonist (IL-1ra) and IL$1 \alpha$. Many biological effects of IL-1 $\beta$ are similar to and shared with those of TNF$\alpha$. IL-1 $\beta$ is also a cytokine mainly produced by activated macrophages, and taking part in the innate immune response in the acute phase of inflammation. However, many other different sources can be responsible for the production of this cytokine, for instance neutrophils, endothelial and epithelial cells. The principal functions of IL-1 $\beta$ as a mediator of the immune response include apoptosis, cell differentiation and proliferation. Caspase-1 (called IL-1 converting enzyme) proteolytically cleaves pro-IL-1 $\beta(33 \mathrm{kD})$ so as to obtain the mature form of IL-1 $\beta(17 \mathrm{kD})$. IL-1 $\beta$, as other IL-1 proteins, binds to the IL-1 receptor type I (IL-1RI). Both, IL-1 $\alpha$ and IL-1 $\beta$ bind to the IL-1 receptor type II (IL-1RII), but no signal transduction is initiated because it does not posses an intracellular domain (Boraschi and Tagliabue, 2006). 
The involvement of IL-1 $\beta$ during chronic pain is primarily related to its interaction with glia, upregulation of other proalgesic agents, and by altering neuronal transmission of nociceptors. Early studies performed in rats demonstrate the hyperalgesic effects of IL-1 $\beta$ after peripheral (Fukuoka et al., 1994) or central (Oka et al., 1993; 1994) administration. In both cases, enhanced responses to mechanical and/or thermal noxious stimuli were recorded following IL-1 $\beta$ injection either into the lateral cerebral ventricle or the hindpaw, evidencing the potent hyperalgesic effect of this cytokine. In a different study, direct application of IL-1 $\beta$ to the lumbar dorsal roots resulted in diminished sensory neural activity, suggesting that this and other cytokines may have a possible neurotoxic effect after chronic exposition (Ozaktay et al., 2002). The same result has for example been obtained with TNF- $\alpha$, but not with IL-6. Further differences between cytokines were illustrated in a study where IL-1 $\beta$ was able to enhance both frequency and amplitude of spontaneous excitatory postsynaptic currents; whereas TNF- $\alpha$ only enhanced the frequency, and IL-6 reduced it (Kawasaki et al., 2008). Electrophysiological recordings of harvested dorsal root ganglia from rats show that IL- $1 \beta$ induces sensitization of sensory neurons to heat, mediated by activation of protein kinases and not by $G$ proteinlinked receptors (Obreja et al., 2002). In the last decade, the interest for the role of microglia in pain has grown. Several reports have studied the relation between glial activation and IL-1 $\beta$ during exaggerated pain behavior. Marked glial activation has been shown after more extensive nerve root injury in rats, demonstrating a positive correlation between the degree of damage and the level of microglial expression (Winkelstein and DeLeo, 2002). However, in a separate study that used the same model, no direct association was established between the severity of pain behavior in animals and the level of microglial activation in the spinal cord (Hashizume et al., 2000).

\subsubsection{Interleukin-6}

IL-6 is a pleiotropic cytokine involved in a wide range of functions on the inflammatory response under pathological circumstances, but almost absent in physiological conditions. It is secreted mainly by macrophages, endothelial and T cells. Primarily, this cytokine exerts its effects on the liver, B cells, muscle cells, neurons and glia, by stimulating the immune response and contributing with proliferation of antibody-producing cells. IL-6 (26kD) is a protein that has been described to possess both proinflammatory and anti-inflammatory characteristics (Wong et al., 2003). This cytokine binds to its receptor which 
consists of a cytokine-binding protein (in a complex with the soluble form of the receptor, sIL-6R) with the so-called gp130 (a subunit for signal transduction), the latter is involved on the signaling transduction for numerous cytokines.

The role of IL- 6 on hyperalgesia is widely associated with muscle pain under circumstances of inflammation (Dina et al., 2008). In a recent study, mechanical hyperalgesia and acute inflammation were induced after intramuscular IL-6 injection into the mice gastrocnemius. Further analyses revealed an elevation of neutrophils in the muscle, and the presence of the cytokine in the DRG. Moreover, intracellular signaling inhibitors were shown to reduce the mechanical nociceptive response. These findings suggest that the initiation and maintenance of muscle-related pain is dependent on resident cell activation, infiltration of polymorphonuclears and activation of intracellular pathways (especially mitogen-activated protein kinases, MAPK) (Manjavachi et al., 2010). In a different study, a IL-6 knock-out mice model exhibited lower nociceptive thresholds to mechanical and thermal stimuli (Xu et al., 1997). Interestingly, in a similar report, mice lacking gp130 also showed reduced inflammatory and pain behavior levels, demonstrating the critical role of this signal transducer on pathological pain (Andratsch et al., 2009). These findings are in line with electrophysiological recordings that demonstrated how IL-6 in conjunction with sIL-6R enhances sensitization in heat-activated sensory neurons (Obreja et al., 2005).

\subsection{Anti-inflammatory cytokines}

\subsubsection{Interleukin-4}

IL-4 is a critical protein (18-20kD) for the adaptive and humoral immunity regulation. It is primarily known by its role on producing IgE antibodies, differentiation of naive $T$ cells to $T_{H} 2$ cells, and inhibition of differentiation of $T_{H} 1$ cells. Its principal source are $T_{H} 2$ cells and mast cells, one of the reasons why it is mainly associated with allergic reactions. Its receptor (IL-4R) is a member of the type I receptor family. It can also bind to IL-13, which biological actions are similar to those above mentioned for IL-4. The role of IL-4 during pain is related to its anti-inflammatory and inhibitory effects on algesic-related cytokines. In an experimental study in rodents, pretreatment with IL-4 reduced the inflammatoryrelated pain behavior induced by intense inflammatory agents' injection (acetic acid and zymosan). In addition, after zymosan injection, TNF- $\alpha$ and IL-1 $\beta$ levels obtained from peritoneal cells of IL-4-treated animals were significantly 
decreased compared to those obtained from the nontreated group (Vale et al., 2003). IL-4 limits hyperalgesia differently on the early and late phases of the inflammatory response, on the former by inhibiting the release of proinflammatory cytokines (TNF- $\alpha$, IL-1 $\beta$ and IL- 8 ) and on the latter by inhibiting the secretion of prostaglandins (Cunha et al., 1999; Liou et al., 2011). Furthermore, in LPS-activated human monocytes, IL-4 not only inhibited the production of IL-1 $\beta$ (and IL-6), but it also upregulated the synthesis of IL-1ra (Fenton et al., 1992; Vannier et al., 1992).

\subsubsection{Interleukin-10}

The principal role of IL-10 is to inhibit immune responses, predominantly those originated from macrophages and dendritic cells. This pleiotropic protein (18kD) has been largely documented as an analgesic cytokine, primarily due to its ability to suppress proinflammatory cytokine production. It is mainly produced by macrophages, $T$ cells and mast cells. The IL-10 receptor belongs to the family of the type II cytokine receptors. Some of its particularities are its downregulating effect on IL-12 (which indirectly inhibits IFN- $\gamma$ ) and its capacity to inhibit the display of foreign antigen complexes through antigen-presenting cells. The analgesic effects of IL-10 were clearly demonstrated in a series of studies where the intrathecal administration of a viral vector, which encoded for $\mathrm{IL}-10$, reversed the effects of induced neuropathic pain in rats after $\mathrm{CCl}$ (Milligan et al., 2005a; Milligan et al., 2005b; Milligan et al., 2006a; Milligan et al., 2006b). Moreover, another study showed that IL-10 intrathecal administration inhibited the expression of TNF- $\alpha$ and IL-1 $\beta$ in lumbar DRGs (Ledeboer et al., 2007). In another study, IL-10 inhibited the hyperalgesic effects induced by the intraplantar injection of an endotoxin in mice. These actions were attributed to the fact that IL-10 administration significantly downregulated the expression of TNF- $\alpha, \mathrm{IL}-1 \beta$ and nerve growth factor in the injected hindpaw (Kanaan et al., 1998). Similar findings were reported on the hyperalgesic responses elicited by carrageenin and bradykinin, attributed by the authors to an inhibition of proinflammatory cytokines and resulting prostaglandins (Poole et al., 1995). 


\subsection{The inflammasome}

The inflammasome is a high-molecular weight complex that works as one of the first lines of defense from the immune response. It forms part of a highly sophisticated system of the innate immunity known as the pattern recognition receptors (PRRs). This group of receptors is composed by the Toll-like receptors, Nod-like receptors (NLRs) and RIG-like helicase receptors. It has been shown that PRRs recognize certain molecular patterns that are expressed by pathogens in order to activate an inflammatory cascade (Stutz et al., 2009). So far, probably one of the most interesting subsets of inflammasomes to investigate in the field of pain are the NLRs. This is mostly due to the fact that this subgroup of receptors is involved in the production of active proinflammatory cytokines such as IL-1 $\beta$ and IL-18 (Martinon et al., 2002, 2006). In particular, the NALP3 inflammasome has been described as a crucial step in the production and release of IL-1 $\beta$ (Stutz et al., 2009). The algesic effects of the inflammasome are primarily related to acute and chronic episodes of pain after activation of the NALP3 by uric acid crystals in humans (Martinon et al., 2006). In a rat fracture model, the data suggest that the activation of the NALP1 inflammasome contributes to nociception and the development of complex regional pain syndrome (CRPS) (Li et al., 2009). Although the studies correlating pain and the inflammasome are scarce to our days, these multiprotein complexes seem as promising therapeutic targets due to their capacity to sense early damage and act accordingly.

We summarize the effects of the most representative inflammatory agents on diverse acute and chronic diseases in humans in Table 1; last section of the thesis.

\section{Inflammation and pain in human studies}

\subsection{Cytokine determination in human tissue biopsies}

To date, several lines of evidence support the direct involvement of cytokines in pain. However, the initial reports performed in humans that measured inflammatory mediators and correlated them with clinical pain scales appeared only at the very end of the 20th century. Similarly to preclinical studies, the results obtained from human data suggest that proinflammatory cytokines play an algesic role in diverse neurological diseases, whereas anti-inflammatory cytokines participate as analgesic agents. Proinflammatory cytokines, amongst 
others, TNF- $\alpha, \mathrm{IL}-1 \beta, \mathrm{IL}-6$ and IL-8, are detectable in patients with painful neurological disorders (e.g. fibromyalgia, complex regional pain syndrome, small fiber neuropathy) and present significant elevated levels compared to healthy subjects (Maes et al., 1999; Üçeyler et al., 2007a; Üçeyler et al., 2010; Wang et al., 2008a). Furthermore, when patients from the same group of neurological disorders are divided into those with and those without painful symptomatology, we can observe a significant upregulation of the proinflammatory cytokines in the patients with pain complaints (Empl et al., 2001; Ludwig et al., 2008; Üçeyler et al., 2007). On the contrary, expression levels of anti-inflammatory cytokines, such as IL-4 and IL-10, were either low in biopsies from patients with painful symptomatology, or increased in biopsies from patients with low pain scores or painless conditions (Alexander et al., 2007; Üçeyler et al., 2007; Üçeyler et al., 2006).

Several studies have reported a correlation between proinflammatory cytokine levels (TNF- $\alpha, \mathrm{IL}-6$ and IL- 8 ) in the herniated intervertebral discs or in the surrounding tissue of the nerve roots and the severity of leg pain (Burke et al., 2002; Brisby et al., 2002; Genevay et al., 2008; Andrade et al., 2011b). In addition, this correlation between presurgical inflammatory levels and pain severity has proven to remain after months of clinical evaluation, even after 1 year postoperatively (Andrade et al., 2011b). This suggests that not only the mechanical pressure applied by the herniated discs is the single responsible for the pain. These findings, combined with the fact that biopsies from painless control patients did not show inflammation, indicate the biochemical effects exerted by the cytokines. In addition to high cytokine levels, other players have been implicated in the pathogenesis of leg pain from herniated discs. Differential expression of TNF- $\alpha$ receptors, TNFR1 and TNFR2, has been shown to induce opposite effects on pain in humans (Andrade et al., 2011b), which is in accordance with studies performed in animals (Schäfers et al., 2008; Zhang et al., 2011).

With respect to CRPS, a large body of evidence has shown the role of inflammatory cytokines in the pathogenesis of the syndrome in humans (Schinkel et al., 2006; Alexander et al., 2005; Maihöfner et al., 2005; Üçeyler et al., 2007a). However, conflicting data still exist about the site of action of these cytokines, so that the question remains whether their effects are produced systemically or locally (Huygen et al., 2002; Krämer et al., 2011). Higher concentrations of the proinflammatory cytokines TNF- $\alpha$, IL-1 $\beta$, IL-2, IL- 6 and IL8 have been found in biopsies from patients with CRPS when they were compared to controls with and without painful conditions (Alexander et al., 
2005, 2007; Krämer et al., 2011; Üçeyler et al., 2007a; Schinkel et al., 2006). In line with these observations, lower concentrations of anti-inflammatory cytokines, such as IL-4 and IL-10, have been found in biopsies from patients with CRPS in a similar experimental design (Alexander et al., 2007; Üçeyler et al., 2007a). Only one study has reported no differences in the production of inflammatory cytokines in CRPS patients when they were compared to healthy controls (van de Beek et al., 2001), suggesting that the pathophysiology of the disease is more likely to come from neurogenic-induced inflammation than from the role played by the immune system. Interestingly, in a different report where the CRPS patients included in the study had dystonia, no differences were found in the CSF levels of various inflammatory mediators between patients and controls (Munts et al., 2008). In this case, the authors did not rule out the possibility that the effects of the inflammatory markers may have taken place in the early phase and that they were not detected at the moment of the study, since their patient population with dystonia had a long duration of the disease. With respect to the spatial distribution of cytokines in CRPS patients, the data obtained from several studies is not consistent in the sense that either a local or a systemic increase of cytokines has been reported (Alexander et al., 2005; Krämer et al., 2011). In one of the first reports addressing this question, blood obtained from suction blisters placed on the affected extremity showed a significant increase of TNF- $\alpha$ and IL-6 levels when it was compared with blood coming from the unaffected extremity (Huygen et al., 2002). This cytokine increase selectively on the affected limb evidenced the local involvement of inflammation in CRPS. In a different study, skin biopsies were compared with blood samples from CRPS patients, osteoarthritis controls scheduled for surgery and bone fracture patients without CRPS (Krämer et al., 2011). Here, the differences between skin and serum TNF- $\alpha$ levels evidenced the local effects of inflammation. In skin biopsies, a higher concentration of TNF- $\alpha$ levels was found in the CRPS patient group when they were compared to the other groups, whereas in serum the concentrations of TNF- $\alpha$ were equivalent between all the groups. It may be important to remark that, in these two reports (Huygen et al., 2002; Krämer et al., 2011), the patients included in the studies were diagnosed as CRPS type 1 only, which by definition does not contain a major nerve lesion. On the other hand, different and more numerous reports point towards a systemic involvement of inflammation in CRPS patients. In these studies, high concentrations of proinflammatory cytokine levels have been found in CSF and blood samples from CRPS patients (Alexander et al., 2005, 2007; Schinkel et al., 2006; Üçeyler et al., 2007a). In one of these studies (Schinkel et al., 2006) a similar experimental study from that published by 
Huygen et al. was performed, where the local expression of cytokines was responsible for the pain. However, in this study, Schinkel et al. reported elevated inflammatory mediators (IL-8, TNFR1 and TNFR2) in blood samples that came from both arms in CRPS patients. These findings support the theory of elevated proinflammatory markers in CRPS, but not the role of a localized inflammatory process. Interestingly, in the majority of the reports where a systemic distribution of cytokines was found, the CPRS patients enrolled in these studies were type 1 and 2 (Alexander et al., 2005; Maihöfner et al., 2005; Üçeyler et al., 2007a). This may relate to the observation that more patients in these studies had larger nerve damage.

The expression of inflammatory cytokines in patients with painful neuropathies follows a similar pattern than the one from CRPS patients. Several authors have suggested that inflammation plays an important role in the pathogenesis of painful neuropathies. However, divergent data suggest that the site of action of these cytokines is systemic or local (Üçeyler et al., 2007b; Üçeyler et al., 2010). It is probable that in the same way that it occurs in other conditions the temporal expression of cytokines can be affected and therefore the detection of these markers may be affected as well. So far, we cannot infer with these contrasting data what is the real distribution of inflammation in patients with painful neuropathies. Nonetheless, we cannot rule out the evident effects created by this process. In terms of inflammation research, one of the biggest advantages when we study neuropathies is that they can be divided into painful and non-painful variants. This facilitates the analysis because all patients have the same condition, in this case the neuropathy, but one variable, which is the symptom of pain. Proinflammatory cytokines such as TNF- $\alpha$, IL-1 $1 \beta$, IL-2, IL-6, TGF- $\beta$, IL- 4 and IL-10 have been recognized to be important factors in the mediation of painful neuropathies. High levels of proinflammatory cytokines, for example, have been identified in blood samples and skin biopsies of patients with painful neuropathies when they were compared to non-painful neuropathies and healthy controls (Üçeyler et al., 2007b; Doupis et al., 2009; Üçeyler et al., 2010). Interestingly, when CSF and blood samples were investigated in these patients, high levels of TNF- $\alpha$ and IL- 6 were found in serum, but not in the CSF samples of patients with severe neuropathies (Ludwig et al., 2008). Moreover, TNF- $\alpha$ levels in blood were increased in patients with mechanical allodynia compared to those who did not. These findings are in accordance with a study where the biopsies were obtained directly from the nerve tissue (Empl et al., 2001), although this study showed no differences between serum TNFR1 levels in patients with painful neuropathies and those samples from patients with non-painful neuropathies. Nevertheless, 
TNFR1 levels were significantly elevated in patients with mechanical allodynia, suggesting that the expression of the receptor might influence central mechanisms of pain. With respect to anti-inflammatory cytokines, fewer discrepancies are reported, showing a systemic increase of IL-4 and IL-10 in blood samples from patients with non-painful neuropathies (Üçeyler et al., $2007 \mathrm{~b}$ ). These findings are in line with the proposed analgesic role of antiinflammatory cytokines (Kraus et al., 2001; Milligan et al., 2005b).

Inflammation and cytokines have been long associated with fibromyalgia, however the correlation with pain has not been that consistent. Although there is a large tendency that fibromyalgia is linked with high levels of proinflammatory cytokines (Wallace et al., 2001; Salemi et al., 2003; Wang et al., 2009; Gür et al., 2002), certain studies have reported no changes between fibromyalgia patients and healthy controls blood samples (Maes et al., 1999; Amel Kashipaz et al., 2003; Wang et al., 2008). Only the proinflammatory cytokines IL-1 $\beta$, IL- 6 and IL-8 have been shown to be increased in skin biopsies and in serum samples from fibromyalgic patients (Salemi et al., 2003; Wang et al., 2009). Similarly, another study reports a reduction in the level of antiinflammatory cytokines (IL-4 and IL-10) in blood samples in patients with fibromyalgia within a population of chronic widespread pain patients (Üçeyler et al., 2006). So far, the discrepancies between reports make the clinical relevance of inflammation in correlation with fibromyalgia related pain uncertain (Üçeyler et al., 2011).

A complete overview of the effects of pro- and anti-inflammatory cytokines on chronic pain disorders in humans is provided in Table 2; last sections of the thesis.

\subsection{Human imaging studies}

Throughout many decades, nuclear medicine techniques have demonstrated sufficient efficacy and sensitivity in the estimation of the inflammatory activity level in several rheumatic diseases. These techniques are primarily based on the systemic application of radiopharmaceuticals and the subsequent imaging of the uptake produced by these agents in specific anatomical areas of interest (for review see (van der Laken et al., 1998)). After years of use and experimentation, various radiopharmaceuticals have been developed to provide information about the location and physiological changes of inflamed tissue. For instance, some of the most recognized agents are radiolabeled leukocytes, 
small proteins and peptides (e.g. IL-1, IL-1ra, IL-2, IL-8, octreotide, substance $\mathrm{P})$, monoclonal antibodies, ${ }^{99 \mathrm{~mm}}$ Tc-labelled nanocolloids, and anti-TNF- $\alpha$ antibodies (Chianelli et al., 2006; van der Laken et al., 1998). The utilization of these techniques as a diagnostic tool in the field of pain is a contemporary story. In a recent report, where an imaging study was performed to detect TNF$\alpha$ in complex regional pain syndrome type 1 patients, the authors demonstrated the capacity of the ${ }^{99 m m}$ Tc-labelled anti-TNF- $\alpha$ antibody (infliximab) to localize affected areas in the early stage of the disease (Bernateck et al., 2010). This data do not only confirm previous molecular findings obtained from tissue biopsies, but also allow us to obtain a direct visualization of the inflammatory process in painful affected structures. Similarly, the application of other radiolabeled anti-TNF- $\alpha$ compounds (infliximab and adalimumab) have been shown to be effective in visualizing affected painful areas in rheumatic patients (Barrera et al., 2003; Conti et al., 2005). In the case of infliximab, 4 months after treatment (also with infliximab, intraarticular administration) the VAS score changed from 52 to 0 points, and the follow-up scan images after therapy showed no uptake of the agent accordingly (Conti et al., 2005). These data also suggest the association between the clinical symptoms at the moment of evaluation and the presence of the inflammatory cytokine on the injured tissue, showing an important temporal connection between inflammation and pain. However, not all the agents have proven to be successful in the detection of inflammation. In an early study where radiolabeled IL-1ra was administered, the binding to IL-1 in painful regions did not show specific targeting (Barrera et al., 2000). Succinctly, the application of radiolabeled agents for the imaging of inflammation in association with pain is still a novel field and is considered in many cases as an experimental procedure. The results obtained by these techniques are promising until now, however the number of patients tested is still modest so more studies will be required in order to include them as conventional diagnostic tools.

\section{Analgesic therapies based on cytokine modulation}

Many studies have reported the use of cytokine modulators to treat inflammatory and non-inflammatory-related pain disorders in both humans and animals (Sommer et al., 2001; Fleischman et al., 2003; Karppinen et al., 2003; Hao et al., 2006). However, a significant number of these clinical reports are not controlled trials or simply do not have a correlation with pain scales. This makes it difficult to establish if a specific therapy offers a real benefit to treat a precise 
pain disorder. Undoubtedly, the most investigated group among these therapies is the TNF- $\alpha$ inhibitors integrated by etanercept, infliximab and adalimumab (Moreland et al., 1997; Korhonen et al., 2004). Recently, in a controlled trial, the authors reported that subcutaneous injections of adalimumab at 7-day intervals significantly reduced the severity of leg pain in patients with lumbar disc herniation (Genevay et al., 2010). Furthermore, in a 3-year follow-up of this study, the data confirmed that a short administration of this TNF- $\alpha$ inhibitor in the early stage of the disease reduces the necessity of surgery in the majority of the patients (Genevay et al., 2011). Other medications like anakinra and tocilizumab have been used to suppress the activity of interleukins in the treatment of rheumatoid arthritis in humans (Fleischman et al., 2003; Maini et al., 2006). These reports suggest that the reduction of IL-1 and IL-6 levels in rheumatic patients lead to remission of the symptoms and therefore decrease of the pain. Many preclinical studies still continue to report promising data on cytokine modulation, but their translation into human therapies is nonetheless far. Especially interesting are gene therapies mediated via the introduction of viruses that promotes the release of anti-inflammatory cytokines (Milligan et al., 2005; Hao et al., 2006). An overview of these modulatory strategies is provided in Table 3; last section of the thesis.

\section{Conclusion and future directions}

There appears to be enough scientific evidence from experimental and clinical studies to support the role of inflammation as a mechanism involved in pain. Recent reports, as described in the present review, suggest that cytokines have a crucial role in the development and maintenance of pain. However, the direct correlation between the expression of these markers and the severity of pain in humans still remains unclear. Therefore we require more controlled trials that apply standardized pain scales to a well defined patient population or specific disorder. So far, it seems as this correlation may exist in certain disorders and that it is present in specific moments during the development of the disease. Nonetheless, we must not forget that most evidence point that an unbalance toward proinflammatory cytokines is necessary to have pain, and on the contrary, a decrease of anti-inflammatory cytokines expression may lead to pain reduction or the abscense of it. These findings however should be taken with caution at the moment of using old therapies and designing new ones, because it should be kept in mind that the pro-inflammatory processes after nerve damage are not exclusively damaging. It is well known that pro-inflammatory 
cytokines are one of the primary sources of regeneration. Therefore, our efforts to prevent chronic pain states should be focused on the modulation of a balanced inflammatory enviroment. We believe that this can only be achieved if we can fulfill the following premises, (1) once we can identify individually those patients in whom their inflammatory profile is associated with pain (either using molecular or imaging tests), and (2) by administering several complimentary modulatory therapies to assure neuroprotection and regeneration.

\section{References}

1. Alexander GM, et al. Changes in immune and glial markers in the CSF of patients with Complex Regional Pain Syndrome. Brain Behav Immun 2007;21:668-76.

2. Alexander GM, et al. Changes in cerebrospinal fluid levels of proinflammatory cytokines in CRPS. Pain 2005;116:213-9.

3. Amel Kashipaz MR, et al. Normal production of inflammatory cytokines in chronic fatigue and fibromyalgia syndromes determined by intracellular cytokine staining in short-term cultured blood mononuclear cells. Clin Exp Immunol 2003;132:360-5.

4. Andrade $P$, et al. Role of TNF-alpha during central sensitization in preclinical studies. Neurol Sci 2011a;32:757-771.

5. Andrade $P$, et al. TNF-alpha levels correlate with postoperative pain severity in lumbar disc hernia patients: Opposite clinical effects between TNF receptor 1 and 2. Pain 2001b;152:2645-2652.

6. Andratsch $\mathrm{M}$, et al. A key role for gp130 expressed on peripheral sensory nerves in pathological pain. J Neurosci 2009;29:13473-83.

7. Arruda JL, et al. Intrathecal anti-IL-6 antibody and IgG attenuates peripheral nerve injury-induced mechanical allodynia in the rat: possible immune modulation in neuropathic pain. Brain Res 2000;879:216-225.

8. Backonja MM, et al. Altered cytokine levels in the blood and cerebrospinal fluid of chronic pain patients. $J$ Neuroimmunol 2008;195:157-63.

9. Baron R. Mechanisms of disease: neuropathic pain--a clinical perspective. Nat Clin Pract Neurol 2006;2:95-106.

10. Baron R, et al. Neuropathic pain: diagnosis, pathophysiological mechanisms, and treatment. Lancet Neurol 2010;9:807-19.

11. Barrera $P$, et al. Scintigraphic detection of tumour necrosis factor in patients with rheumatoid arthritis. Ann Rheum Dis 2003;62:825-8. 
12. Barrera $P$, et al. Radiolabelled interleukin-1 receptor antagonist for detection of synovitis in patients with rheumatoid arthritis. Rheumatology (Oxford) 2000;39:870-4.

13. Basbaum Al, et al. Cellular and molecular mechanisms of pain. Cell 2009;139:267-84.

14. Bennett GJ, Xie YK. A peripheral mononeuropathy in rat that produces disorders of pain sensation like those seen in man. Pain 1988;33:87107.

15. Berger JV, et al. Enhanced neuroinflammation and pain hypersensitivity after peripheral nerve injury in rats expressing mutated superoxide dismutase 1. J Neuroinflammation 2011;8:33.

16. Bernateck $M$, et al. The first scintigraphic detection of tumor necrosis factor-alpha in patients with complex regional pain syndrome type 1. Anesth Analg 2010;110:211-5.

17. Boraschi $D$, Tagliabue A. The interleukin-1 receptor family. Vitam Horm 2006;74:229-54.

18. Brisby $\mathrm{H}$, et al. Proinflammatory cytokines in cerebrospinal fluid and serum in patients with disc herniation and sciatica. Eur Spine $J$ 2002;11:62-6.

19. Burke JG, et al. Intervertebral discs which cause low back pain secrete high levels of proinflammatory mediators. J Bone Joint Surg $\mathrm{Br}$ 2002;84:196-201.

20. Chen G, Goeddel DV. TNF-R1 signaling: a beautiful pathway. Science 2002;296:1634-1635.

21. Chianelli $M$, et al. New radiopharmaceuticals for imaging rheumatoid arthritis. Q J Nucl Med Mol Imaging 2006;50:217-25.

22. Conti $F$, et al. Successful treatment with intraarticular infliximab for resistant knee monarthritis in a patient with spondylarthropathy: a role for scintigraphy with 99mTc-infliximab. Arthritis Rheum 2005;52:1224-6.

23. Cunha $F Q$, et al. Cytokine-mediated inflammatory hyperalgesia limited by interleukin-4. Br J Pharmacol 1999;126:45-50.

24. Czeschik JC, et al. TNF-alpha differentially modulates ion channels of nociceptive neurons. Neurosci Lett 2008;434:293-8.

25. Dina OA, et al. Role of interleukin- 6 in chronic muscle hyperalgesic priming. Neuroscience 2008;152:521-5.

26. Doupis $\mathrm{J}$, et al. Microvascular reactivity and inflammatory cytokines in painful and painless peripheral diabetic neuropathy. J Clin Endocrinol Metab 2009;94:2157-63. 
27. Echeverry $S$, et al. Transforming growth factor-beta 1 impairs neuropathic pain through pleiotropic effects. Mol Pain 2009;5:16.

28. Empl M, et al. TNF-alpha expression in painful and nonpainful neuropathies. Neurology 2001;56:1371-7.

29. Faustman D, Davis M. TNF receptor 2 pathway: drug target for autoimmune diseases. Nat Rev Drug Discov 2010;9:482-93.

30. Fenton $\mathrm{MJ}$, et al. IL-4 reciprocally regulates IL-1 and IL-1 receptor antagonist expression in human monocytes. J Immunol 1992;149:12838.

31. Fleischmann RM, et al. Anakinra, a recombinant human interleukin-1 receptor antagonist (r-metHulL-1ra), in patients with rheumatoid arthritis: A large, international, multicenter, placebo-controlled trial. Arthritis Rheum 2003;48:927-34.

32. Fukuoka $\mathrm{H}$, et al. Cutaneous hyperalgesia induced by peripheral injection of interleukin-1 beta in the rat. Brain Res 1994;657:133-40.

33. Genevay $S$, et al. Elevated levels of tumor necrosis factor-alpha in periradicular fat tissue in patients with radiculopathy from herniated disc. Spine 2008;33:2041-6.

34. George A, et al. Tumor necrosis factor receptor 1 and 2 proteins are differentially regulated during Wallerian degeneration of mouse sciatic nerve. Exp Neurol 2005;192:163-6.

35. Gold MS, et al. Modulation of TTX-R INa by PKC and PKA and their role in PGE2-induced sensitization of rat sensory neurons in vitro. $J$ Neurosci 1998;18:10345-55.

36. Gür $A$, et al. Cytokines and depression in cases with fibromyalgia. J Rheumatol 2002;29:358-61.

37. Hao S, et al. HSV-mediated expression of interleukin-4 in dorsal root ganglion neurons reduces neuropathic pain. Mol Pain 2006;2:6.

38. Hashizume $\mathrm{H}$, et al. Spinal glial activation and cytokine expression after lumbar root injury in the rat. Spine 2000;25:1206-17.

39. Hatashita $S$, et al. Contralateral neuropathic pain and neuropathology in dorsal root ganglion and spinal cord following hemilateral nerve injury in rats. Spine 2008;33:1344-51.

40. Heffner $\mathrm{KL}$, et al. Chronic low back pain, sleep disturbance, and interleukin-6. Clin J Pain 2011;27:35-41.

41. Huygen FJ, et al. Evidence for local inflammation in complex regional pain syndrome type 1. Mediators Inflamm. 2002;11:47-51.

42. IASP. Classification of chronic pain. Descriptions of chronic pain syndromes and definitions of pain terms. Prepared by the International 
Association for the Study of Pain, Subcommittee on Taxonomy. Pain 1986;24:S1-226.

43. Igarashi $\mathrm{A}$, et al. Correlation between inflammatory cytokines released from the lumbar facet joint tissue and symptoms in degenerative lumbar spinal disorders. J Orthop Sci 2007;12:154-60.

44. Ji RR, et al. Central sensitization and LTP: do pain and memory share similar mechanisms? Trends Neurosci 2003;26:696-705.

45. Ji RR, et al. p38 MAPK activation by NGF in primary sensory neurons after inflammation increases TRPV1 levels and maintains heat hyperalgesia. Neuron 2002;36:57-68.

46. Kanaan SA, et al. Interleukin-10 reduces the endotoxin-induced hyperalgesia in mice. J Neuroimmunol 1998;86:142-50.

47. Karppinen J, et al. Tumor necrosis factor-alpha monoclonal antibody, infliximab, used to manage severe sciatica. Spine 2003;28:750-3.

48. Kawasaki $\mathrm{Y}$, et al. Cytokine mechanisms of central sensitization: distinct and overlapping role of interleukin-1beta, interleukin-6, and tumor necrosis factor-alpha in regulating synaptic and neuronal activity in the superficial spinal cord. J Neurosci 2008;28:5189-94.

49. Kim SH, Chung JM. An experimental model for peripheral neuropathy produced by segmental spinal nerve ligation in the rat. Pain 1992;50:355-63.

50. Koch A, et al. Nitric oxide and pro-inflammatory cytokines correlate with pain intensity in chronic pain patients. Inflamm Res 2007;56:32-7.

51. Koike $\mathrm{H}$, et al. Neuropathic pain correlates with myelinated fibre loss and cytokine profile in POEMS syndrome. J Neurol Neurosurg Psychiatry 2008;79:1171-9.

52. Korhonen $\mathrm{T}$, et al. Efficacy of infliximab for disc herniation-induced sciatica: one-year follow-up. Spine 2004;29:2115-9.

53. Krämer HH, et al. TNF-alpha in CRPS and 'normal' trauma - Significant differences between tissue and serum. Pain 2011;152:285-90.

54. Kraus $\mathrm{J}$, et al. Regulation of mu-opioid receptor gene transcription by interleukin-4 and influence of an allelic variation within a STAT6 transcription factor binding site. J Biol Chem 2001;276:43901-8.

55. Kraychete DC, et al. Proinflammatory cytokines in patients with neuropathic pain treated with Tramadol. Rev Bras Anestesiol 2009;59:297-303.

56. Kraychete DC, et al. Serum cytokine levels in patients with chronic low back pain due to herniated disc: analytical cross-sectional study. Sao Paulo Med J 2011;128:259-62. 
57. Kwon BK, et al. Cerebrospinal fluid inflammatory cytokines and biomarkers of injury severity in acute human spinal cord injury. J Neurotrauma 2010;27:669-82.

58. Larson $\mathrm{AA}$, et al. Pain threshold changes in adjuvant-induced inflammation: a possible model of chronic pain in the mouse. Pharmacol Biochem Behav 1986;24:49-53.

59. Ledeboer $A$, et al. Intrathecal interleukin-10 gene therapy attenuates paclitaxel-induced mechanical allodynia and proinflammatory cytokine expression in dorsal root ganglia in rats. Brain Behav Immun 2007;21:686-98.

60. Lee HL, et al. Temporal expression of cytokines and their receptors mRNAs in a neuropathic pain model. Neuroreport 2004;15:2807-11.

61. Leung L, Cahill CM. TNF-alpha and neuropathic pain--a review. J Neuroinflammation 2010;7:27.

62. Lindenlaub T, Sommer C. Cytokines in sural nerve biopsies from inflammatory and non-inflammatory neuropathies. Acta Neuropathol 2003;105:593-602.

63. Li WW, et al. The NALP1 inflammasome controls cytokine production and nociception in a rat fracture model of complex regional pain syndrome. Pain 2009;147:277-86.

64. Liou JT, et al. Inflammation confers dual effects on nociceptive processing in chronic neuropathic pain model. Anesthesiology 2011;114:660-72.

65. Liu $\mathrm{YL}$, et al. Tumor necrosis factor-alpha induces long-term potentiation of $\mathrm{C}$-fiber evoked field potentials in spinal dorsal horn in rats with nerve injury: the role of NF-kappa B, JNK and p38 MAPK. Neuropharmacology 2007;52:708-15.

66. Ludwig J, et al. Cytokine expression in serum and cerebrospinal fluid in non-inflammatory polyneuropathies. J Neurol Neurosurg Psychiatry 2008;79:1268-73.

67. Maes $M$, et al. The immune-inflammatory pathophysiology of fibromyalgia: increased serum soluble gp130, the common signal transducer protein of various neurotrophic cytokines. Psychoneuroendocrinology 1999;24:371-83.

68. Maihofner $\mathrm{C}$, et al. Mechanical hyperalgesia in complex regional pain syndrome: a role for TNF-alpha? Neurology 2005;65:311-3.

69. Maini RN, et al. Double-blind randomized controlled clinical trial of the interleukin-6 receptor antagonist, tocilizumab, in European patients with 
rheumatoid arthritis who had an incomplete response to methotrexate. Arthritis Rheum 2006;54:2817-2829.

70. Manjavachi MN, et al. Mechanisms involved in IL-6-induced muscular mechanical hyperalgesia in mice. Pain 2010;151:345-55.

71. Martinon F, et al. Gout-associated uric acid crystals activate the NALP3 inflammasome. Nature 2006;440:237-41.

72. Martinon $\mathrm{F}$, et al. The inflammasome: a molecular platform triggering activation of inflammatory caspases and processing of prolL-beta. Mol Cell 2002;10:417-26.

73. McNamee KE, et al. IL-17 induces hyperalgesia via TNF-dependent neutrophil infiltration. Pain 2011;152:1838-45.

74. Milligan ED, et al. Controlling pathological pain by adenovirally driven spinal production of the anti-inflammatory cytokine, interleukin-10. Eur $\mathrm{J}$ Neurosci 2005a;21:2136-48.

75. Milligan ED, et al. Intrathecal HIV-1 envelope glycoprotein gp120 induces enhanced pain states mediated by spinal cord proinflammatory cytokines. J Neurosci 2001;21:2808-19.

76. Milligan ED, et al. Controlling neuropathic pain by adeno-associated virus driven production of the anti-inflammatory cytokine, interleukin-10. Mol Pain 2005b;1:9.

77. Milligan ED, et al. Repeated intrathecal injections of plasmid DNA encoding interleukin-10 produce prolonged reversal of neuropathic pain. Pain 2006a;126:294-308.

78. Milligan ED, et al. Intrathecal polymer-based interleukin-10 gene delivery for neuropathic pain. Neuron Glia Biol 2006b;2:293-308.

79. Moreland LW, et al. Treatment of rheumatoid arthritis with a recombinant human tumor necrosis factor receptor (p75)-Fc fusion protein. N Engl J Med 1997;337:141-7.

80. Munts AG, et al. Analysis of cerebrospinal fluid inflammatory mediators in chronic complex regional pain syndrome related dystonia. Clin J Pain 2008;24:30-4.

81. Obreja $\mathrm{O}$, et al. Fast modulation of heat-activated ionic current by proinflammatory interleukin 6 in rat sensory neurons. Brain 2005;128:1634-41.

82. Obreja $\mathrm{O}$, et al. IL-1 beta potentiates heat-activated currents in rat sensory neurons: involvement of IL-1RI, tyrosine kinase, and protein kinase C. FASEB J 2002;16:1497-503.

83. Ohtori $S$, et al. Proinflammatory cytokines in the cerebrospinal fluid of patients with lumbar radiculopathy. Eur Spine J 2011;20:942-6. 
84. Oka $\mathrm{T}$, et al. Intracerebroventricular injection of interleukin-1 beta induces hyperalgesia in rats. Brain Res 1993;624:61-8.

85. Oka $\mathrm{T}$, et al. Intracerebroventricular injection of interleukin-1 beta enhances nociceptive neuronal responses of the trigeminal nucleus caudalis in rats. Brain Res 1994;656:236-44.

86. Onda A, et al. Exogenous tumor necrosis factor-alpha induces abnormal discharges in rat dorsal horn neurons. Spine 2002;27:161824.

87. Ozaktay AC, et al. Dorsal root sensitivity to interleukin-1 beta, interleukin-6 and tumor necrosis factor in rats. Eur Spine $\mathrm{J}$ 2002;11:467-75.

88. Ozaktay AC, et al. Effects of interleukin-1 beta, interleukin-6, and tumor necrosis factor on sensitivity of dorsal root ganglion and peripheral receptive fields in rats. Eur Spine J 2006;15:1529-37.

89. Poole $\mathrm{S}$, et al. Cytokine-mediated inflammatory hyperalgesia limited by interleukin-10. Br J Pharmacol 1995;115:684-8.

90. Sacerdote $P$, et al. Transient early expression of TNF-alpha in sciatic nerve and dorsal root ganglia in a mouse model of painful peripheral neuropathy. Neurosci Lett 2008;436:210-3.

91. Sakuma $Y$, et al. Up-regulation of p55 TNF alpha-receptor in dorsal root ganglia neurons following lumbar facet joint injury in rats. Eur Spine $\mathrm{J}$ 2007;16:1273-8.

92. Salemi S, et al. Detection of interleukin 1beta (IL-1beta), IL-6, and tumor necrosis factor-alpha in skin of patients with fibromyalgia. J Rheumatol 2003;30:146-50.

93. Schäfers $M$, et al. Increased sensitivity of injured and adjacent uninjured rat primary sensory neurons to exogenous tumor necrosis factor-alpha after spinal nerve ligation. J Neurosci 2003a;23:3028-38.

94. Schäfers $M$, et al. Selective stimulation of either tumor necrosis factor receptor differentially induces pain behavior in vivo and ectopic activity in sensory neurons in vitro. Neuroscience 2008;157:414-23.

95. Schäfers M, Sorkin L. Effect of cytokines on neuronal excitability. Neurosci Lett 2008;437:188-93.

96. Schäfers $M$, et al. Spinal nerve ligation induces transient upregulation of tumor necrosis factor receptors 1 and 2 in injured and adjacent uninjured dorsal root ganglia in the rat. Neurosci Lett 2003b;347:17982.

97. Schäfers $M$, et al. Intramuscular injection of tumor necrosis factor-alpha induces muscle hyperalgesia in rats. Pain 2003c;104:579-88. 
98. Schinkel C, et al. Inflammatory mediators are altered in the acute phase of posttraumatic complex regional pain syndrome. Clin $\mathrm{J}$ Pain 2006;22:235-9.

99. Scuderi GJ, et al. Cytokine assay of the epidural space lavage in patients with lumbar intervertebral disk herniation and radiculopathy. $J$ Spinal Disord Tech 2006;19:266-9.

100. Seltzer Z, et al. A novel behavioral model of neuropathic pain disorders produced in rats by partial sciatic nerve injury. Pain 1990;43:205-18.

101. Smith $\mathrm{BH}$, et al. Chronic pain in primary care. Fam Pract 1999;16:475-82.

102. Singh JA, et al. Tocilizumab for rheumatoid arthritis: a Cochrane systematic review. J Rheumatol 2011;38:10-20.

103. Sommer C, et al. Etanercept reduces hyperalgesia in experimental painful neuropathy. J Peripher Nerv Syst 2001;6:67-72.

104. Song XJ, et al. Mechanical and thermal hyperalgesia and ectopic neuronal discharge after chronic compression of dorsal root ganglia. J Neurophysiol 1999;82:3347-58.

105. Sorkin LS, Doom CM. Epineurial application of TNF elicits an acute mechanical hyperalgesia in the awake rat. J Peripher Nerv Syst 2000;5:96-100.

106. Sorkin LS, Yaksh TL. Behavioral models of pain states evoked by physical injury to the peripheral nerve. Neurotherapeutics 2009;6:609-19.

107. Stutz A, et al. Inflammasomes: too big to miss. J Clin Invest 2009;119:3502-11.

108. Sweitzer S, et al. Intrathecal interleukin-1 receptor antagonist in combination with soluble tumor necrosis factor receptor exhibits an antiallodynic action in a rat model of neuropathic pain. Neuroscience 2001;103:529-39.

109. Üçeyler N, et al. Differential expression patterns of cytokines in complex regional pain syndrome. Pain 2007a;132:195-205.

110. Üçeyler $\mathrm{N}$, et al. Systematic review with meta-analysis: cytokines in fibromyalgia syndrome. BMC Musculoskelet Disord 2011;12:245.

111. Üçeyler $\mathrm{N}$, et al. Elevated proinflammatory cytokine expression in affected skin in small fiber neuropathy. Neurology 2010;74:1806-13.

112. Üçeyler $\mathrm{N}$, et al. Differential expression of cytokines in painful and painless neuropathies. Neurology 2007b;69:42-9. 
113. Üçeyler $\mathrm{N}$, et al. Mode of action of cytokines on nociceptive neurons. Exp Brain Res 2009;196:67-78.

114. Üçeyler N, Sommer C. Cytokine regulation in animal models of neuropathic pain and in human diseases. Neurosci Lett 2008;437:1948.

115. Üçeyler N, et al. Reduced levels of antiinflammatory cytokines in patients with chronic widespread pain. Arthritis Rheum 2006;54:2656-64.

116. Vale ML, et al. Antinociceptive effects of interleukin-4, -10 , and -13 on the writhing response in mice and zymosan-induced knee joint incapacitation in rats. J Pharmacol Exp Ther 2003;304:102-8.

117. van de Beek WJ, et al. Innate cytokine profile in patients with complex regional pain syndrome is normal. Pain 2001;91:259-61.

118. van der Laken CJ, et al. Scintigraphic detection of infection and inflammation: new developments with special emphasis on receptor interaction. Eur J Nucl Med 1998;25:535-46.

119. Vannier E, et al. Coordinated antiinflammatory effects of interleukin 4: interleukin 4 suppresses interleukin 1 production but upregulates gene expression and synthesis of interleukin 1 receptor antagonist. Proc Natl Acad Sci USA 1992;89:4076-80.

120. Verri WA Jr., et al. Hypernociceptive role of cytokines and chemokines: targets for analgesic drug development? Pharmacol Ther 2006;112:116-38.

121. Vogel $C$, et al. Altered pain behavior and regeneration after nerve injury in TNF receptor deficient mice. J Peripher Nerv Syst 2006;11:294-303.

122. Wagner R, Myers RR. Endoneurial injection of TNF-alpha produces neuropathic pain behaviors. Neuroreport 1996;7:2897-901.

123. Wallace DJ, et al. Cytokines play an aetiopathogenetic role in fibromyalgia: a hypothesis and pilot study. Rheumatology (Oxford) 2001;40:743-9.

124. Wang $\mathrm{H}$, et al. The role of IL-8 in patients with fibromyalgia: a prospective longitudinal study of 6 months. Clin J Pain 2009;25:1-4.

125. Wang $\mathrm{H}$, et al. Circulating cytokine levels compared to pain in patients with fibromyalgia -- a prospective longitudinal study over 6 months. J Rheumatol 2008a;35:1366-70.

126. Wang $\mathrm{H}$, et al. The role of TNF-alpha in patients with chronic low back pain-a prospective comparative longitudinal study. Clin J Pain 2008b;24:273-8. 
127. Watkins LR, et al. Immune activation: the role of proinflammatory cytokines in inflammation, illness responses and pathological pain states. Pain 1995;63:289-302.

128. Watkins LR, et al. Glial activation: a driving force for pathological pain. Trends Neurosci. 2001a;24:450-5.

129. Watkins LR, et al. Spinal cord glia: new players in pain. Pain 2001b;93:201-5.

130. Winkelstein BA, DeLeo JA. Nerve root injury severity differentially modulates spinal glial activation in a rat lumbar radiculopathy model: considerations for persistent pain. Brain Res 2002;956:294-301.

131. Wong PK, et al. The role of the interleukin-6 family of cytokines in inflammatory arthritis and bone turnover. Arthritis Rheum 2003;48:1177-89.

132. $\mathrm{Xu} \mathrm{XJ}$, et al. Nociceptive responses in interleukin-6-deficient mice to peripheral inflammation and peripheral nerve section. Cytokine 1997;9:1028-33.

133. Youn $\mathrm{DH}$, et al. Exogenous tumor necrosis factor-alpha rapidly alters synaptic and sensory transmission in the adult rat spinal cord dorsal horn. J Neurosci Res 2008;86:2867-75.

134. Zeilhofer $\mathrm{HU}$, et al. Chronic pain states: pharmacological strategies to restore diminished inhibitory spinal pain control. Annu Rev Pharmacol Toxicol 2012;52:111-33.

135. Zelenka $\mathrm{M}$, et al. Intraneural injection of interleukin-1beta and tumor necrosis factor-alpha into rat sciatic nerve at physiological doses induces signs of neuropathic pain. Pain 2005;116:257-63.

136. Zhang L, et al. TNF-alpha contributes to spinal cord synaptic plasticity and inflammatory pain: Distinct role of TNF receptor subtypes 1 and 2. Pain 2011;152:419-27.

137. Zheng W, et al. Glial TNFa in the spinal cord regulates neuropathic pain induced by HIV gp120 application in rats. Mol Pain 2011;7:40.

138. Zhou $Z$, et al. A novel cell-cell signaling by microglial transmembrane TNFa with implications for neuropathic pain. Pain 2010;151:296-306. 


\section{Chapter 4}

Tumor necrosis factor- $\alpha$ levels correlate with postoperative pain severity in lumbar disc hernia patients: opposite clinical effects between TNF receptor 1 and 2

Pablo Andrade, Veerle Visser-Vandewalle, Marjan Philippens, Marc A. Daemen, Harry W.M. Steinbusch, Wim A. Buurman, Govert Hoogland 


\section{Chapter 4}

\section{Abstract}

Lumbar disc hernia (LDH) is a leading cause for chronic pain in adults. The underlying pathology of chronic pain following discectomy remains unclear. Chronic local inflammation is considered to underlie painful symptomatology. In this context we investigated TNF- $\alpha$, TNFR1 and TNFR2 expression at the moment of surgery in LDH patients and correlate it with the severity of postoperative pain. We analyzed protein and mRNA levels from muscle, ligamentum flavum (LF), annulus fibrosus (AF) and nucleus pulposus (NP) in LDH patients and scoliosis patients (SP), which served as controls. Pain assessment with the visual analogue scale (VAS) was performed 1 day before surgery, and 6 weeks and 12 months postoperatively. TNF- $\alpha$ protein levels were detected in AF, LF and NP in all LDH patients, but not in SP. TNF-a mRNA was significantly higher in LDH patients than in SP; i.e. 5-fold in AF, 3fold in NP and 2-fold in LF. For the NP, TNF- $\alpha$ protein levels correlated with VAS scores ( $r=0.54$ at 6 weeks and $r=0.65$ at 12 months follow-up). Also, TNFR1 protein levels in NP positively correlated with VAS scores $(r=0.75$ at 6 weeks and $r=0.80$ at 12 months follow-up). Whereas, TNFR2 protein levels in AF negatively correlated with VAS scores $(r=-0.67$ at 6 weeks and $r=-0.67$ at 12 months follow-up). These data indicate that TNF-a levels could determine the clinical outcome in LDH patients following discectomy. Moreover, the opposite correlation of TNF receptors with pain sensation suggests that an unbalanced expression plays a role in the generation of pain. 
TNF-alpha levels correlate with postoperative pain in LDH patients

\section{Introduction}

Low back pain is one of the most common causes of chronic pain in adults. It represents an enormous social and economic burden with a prevalence of up to $46 \%$ of the population, of which $28 \%$ consist of severe cases [10]. Lumbar disc herniation (LDH) is one of the leading causes of low back pain, whereby compressed nerve roots at their exit from the spinal column form a lesion responsible for the symptomatology at the constriction site and along the trajectory of the affected nerve (e.g. sciatica). Conventional therapy comprises analgesics, anti-inflammatory drugs and physiotherapy. In the majority of these cases chronic pain can be treated without surgery. However, many patients still experience pain and disability one year after consultation with their physician [6]. For LDH patients with persistent pain, decompressive surgery (discectomy) is often considered a good option to relieve pain. Nevertheless, around 10 to $30 \%$ of patients that undergo elective discectomy report unsatisfactory results after months and years of evaluation [3, 4, 29]. The underlying mechanisms of chronic sciatic pain following discectomy are still unclear.

The interaction between the nervous and immune systems is considered an essential element for the development and perpetuation of pain. Various cytokines have been associated to contribute in the sensation of pain; among these, tumor necrosis factor-alpha (TNF- $\alpha$ ) is considered to act as a regulatory player in induction of pain in the peripheral and central nervous system. TNF- $\alpha$ has been demonstrated to be a powerful inducer of an increase sensitivity to pain (hyperalgesia) when exogenously applied [36, 42]. TNF- $\alpha$ is well known for its role in orchestrating immune responses in reaction to tissue injury including cellular death, survival, proliferation, differentiation and controlling the release of other cytokines [43]. The broad role of TNF- $\alpha$ action in the regulation of the immune response is not yet fully understood. One feasible explanation is the capability of TNF- $\alpha$ to bind to two different receptors which can activate different signaling pathways [1,11]. These receptors are located at the cell membrane and consist of the constitutive TNF receptor 1 (TNFR1 or p55) and the restricted inducible TNF receptor 2 (TNFR2 or p75) [20, 21, 41]. Both receptors display diverse mechanisms of crosstalk and independent activation, ultimately resulting in apoptosis or cell survival [11,26]. How these pathways affect pain sensation hitherto is not understood. More specifically, in the case of LDH surgery, the question remains why some patients suffer from persistent postoperative pain and others not, after a relatively simple standard operation, even when performed in exactly the same circumstances, by the same surgeon. 


\section{Chapter 4}

The aim of this study was to assess the correlation between TNF- $\alpha$ and its receptors and the severity of leg pain after LDH surgery.

\section{Methods}

\subsection{Patients and tissue source}

A total of 15 patients who underwent elective spinal surgery were enrolled in this study. Two different groups were included; group 1 consisted of ten patients who underwent surgery for LDH, and group 2 consisted of five patients who underwent surgery for idiopathic scoliosis, without a history of pain. Scoliosis patients were selected for this study since no degenerative histological changes were observed in the tissue involved, and because this condition offers a control group of painless subjects without inflammation. From each patient nucleus pulposus (NP), annulus fibrosus (AF), ligamentum flavum (LF) and paravertebral muscle biopsies were collected during surgery. NP and AF were macroscopically dissected and excluded the transition zone between both tissues. Patients were suspended anti-inflammatory medication for 7 days before surgery. All samples were immediately frozen in liquid nitrogen and then stored at $-80^{\circ} \mathrm{C}$. All experiments in this study were approved by the Maastricht University Medical Center Ethics Committee.

\subsection{Pain assessment}

Patients diagnosed with LDH were evaluated using the Visual Analogue Scale (VAS) to assess their level of discomfort in the leg at different time points. The scale was applied one day before the operation, and 6 weeks and 12 months after the surgical procedure. In all patients, pain symptoms were considered to be caused by a single-disc disorder. Cauda equina syndrome cases were excluded from this study. In every case, the spinal cord level responsible of the symptomatology was determined through clinical symptomatology, physical examination and imaging findings obtained from magnetic resonance imaging (MRI) of the lumbar spine. 
TNF-alpha levels correlate with postoperative pain in LDH patients

\subsection{Immunoblotting}

All tissue biopsies were cut in two parts, one for quantitative polymerase chain reaction analysis (qPCR, see below) and one for immunoblotting. Immunoblotting samples were homogenized in lysis buffer (phosphate buffered saline (PBS) containing 1\% Igepal, 0.1\% Triton X-100, $1 \mathrm{mM}$ EDTA, $1 \mathrm{mM}$ EGTA, and complete protease inhibitor cocktail (Roche Diagnostics, IN)). Proteins were separated by SDS-10\% polyacrylamide gel electrophoresis for 1.5 hours at $100 \mathrm{~V}$. After this, proteins were transferred to a pure nitrocellulose membrane (Bio-Rad Laboratories, CA) for 1 hour at 100V. Next, membranes were rinsed with PBS, blocked in 1:2 blocking buffer (Odyssey blocking buffer. LI-COR Biosciences, NE): PBS for 1 hour and overnight incubated with primary antibody diluted in $1: 1$ blocking buffer: PBS at $4^{\circ} \mathrm{C}$. Primary antibodies used included: monoclonal mouse-anti human TNF- $\alpha$ (1:100; clone 52B83, Dr. W. Buurman, MUMC, the Netherlands), polyclonal rabbit-anti human TNF-R1 (1:500; H-271, sc-7895, Santa Cruz Biotechnology, CA), polyclonal rabbit-anti human TNF-R2 (1:500; H-202, sc-7862, Santa Cruz Biotechnology), and monoclonal mouse-anti human $\beta$-Actin (1:500; ACTBD11B7, sc-81178, Santa Cruz Biotechnology). Subsequently, membranes were incubated for 1 hour at room temperature with 1:10.000 Alexa Fluor 680 donkey anti-mouse IgG or 1:10.000 Alexa Fluor 800 goat anti-rabbit IgG (Molecular Probes, OR), rinsed with PBS, and developed for enhanced chemiluminescence detection (Odyssey Infrared Imaging System. LI-COR Biosciences). Immunoreactive bands were quantified by calculating the optical density ratio of the band of interest over that of $\beta$-Actin, using a digital imaging analysis system (ImageJ 1.42q National Institute of Health, MD).

\subsection{Quantitative polymerase chain reaction (qPCR)}

Total RNA was isolated from the biopsies using TRIzol (Invitrogen, CA). Next, cDNA was generated from $1 \mu \mathrm{g}$ mRNA using a First Strand cDNA Synthesis Kit (Fermentas International Inc., Ontario, Canada). TNF- $\alpha$, TNFR1 and TNFR2 mRNA expression were measured by qPCR in a LightCycler 480 Real-Time PCR System using SYBR Green LightCycler 480 SYBR Green I Master (Roche Diagnostics) for 50 cycles in a fixed sequence $94^{\circ} \mathrm{C}$ for $30 \mathrm{~s}, 63^{\circ} \mathrm{C}$ for $15 \mathrm{~s}$, and $72^{\circ} \mathrm{C}$ for $15 \mathrm{~s}$. Amplicons were generated using the following primer sets: TNF- $\alpha$-forward (5'-TTC CTG ATC GTG GCA GGC-3') and reverse (5'- GCT GAT TAG AGA GAG GTC CCT G-3'), TNFR1-forward (5'-ACC AAG TGC CAC 
AAA GGA AC-3') and reverse (5'-CTG CAA TTG AAG CAC TGG AA-3'), and TNFR2-forward (5'-TTC GCT CTT CCA GTT GGA CT-3') and reverse (5'-CAC CAG GGG AAG AAT CTG AG-3'). Glyceraldehyde 3-phosphate dehydrogenase (GAPDH) was used as the housekeeping gene to normalize the copy number of the different primers; forward (5'-ACA GTC AGC CGC ATC TTC-3') and reverse (5'-CCA ATA CGA CCA AAT CCG TTG-3'). Melting curve analysis and $2 \%$ agarose gel were performed to verify product specificity. Quantification was performed with the delta CT method using the mentioned reference gene.

\subsection{Statistical analysis}

Data obtained from molecular techniques were expressed as mean \pm SEM and statistically tested using SPSS 17 with a $p$-value $<0.05$ considered as significant. Group differences in protein expression, i.e. $\beta$-Actin normalized optical density values, were evaluated by a Student's $t$-test. Linear regression analysis was used to estimate the correlation between VAS scores and the expression of TNF- $\alpha$ and TNF- $\alpha$ receptors. Differences in mRNA expression were analyzed by a Kruskal-Wallis test.

\section{Results}

\subsection{Patients and pain assessment}

Demographic and clinical data from all LDH patients are presented in Table 1 (last section of the thesis). In all cases the side of the hernia corresponded with the side of the sciatic pain. After imaging and clinical examinations, the distribution of hernias was left-sided on $30 \%$ of the cases, and $70 \%$ right-sided. The affected disc was localized at L3-L4 in 3 cases, L4-L5 in 6 cases and L5-S1 in 1 case. A sequestered hernia was found in $40 \%$ of the patients, while the other $60 \%$ presented a bulging hernia, with black disc disease in $40 \%$ of the cases. Pain intensity baseline according the VAS one day before surgery ranged from 4.5 to 8.4 points (median of 6.7). Delta improvement in the VAS 6 weeks after discectomy ranged from 0.1 to 6.4 points (median of 3 ), expressed in percentage from 1 to $95 \%$ (mean of $55 \%$ ). After 12 months follow-up the delta versus baseline measurements ranged from -1.3 to 6.4 points (median of 3.5 ), expressed in percentage from -19 to $100 \%$ (mean of $43 \%$ ). If the group is 
divided with a delta below or above 4 VAS points, at 6 weeks follow-up 5 patients improved and after 12 months follow-up 4 patients recovered. At 6 weeks and 12 months, there was no correlation between the type of hernia or the absence/ presence of black disc disease and the VAS score.

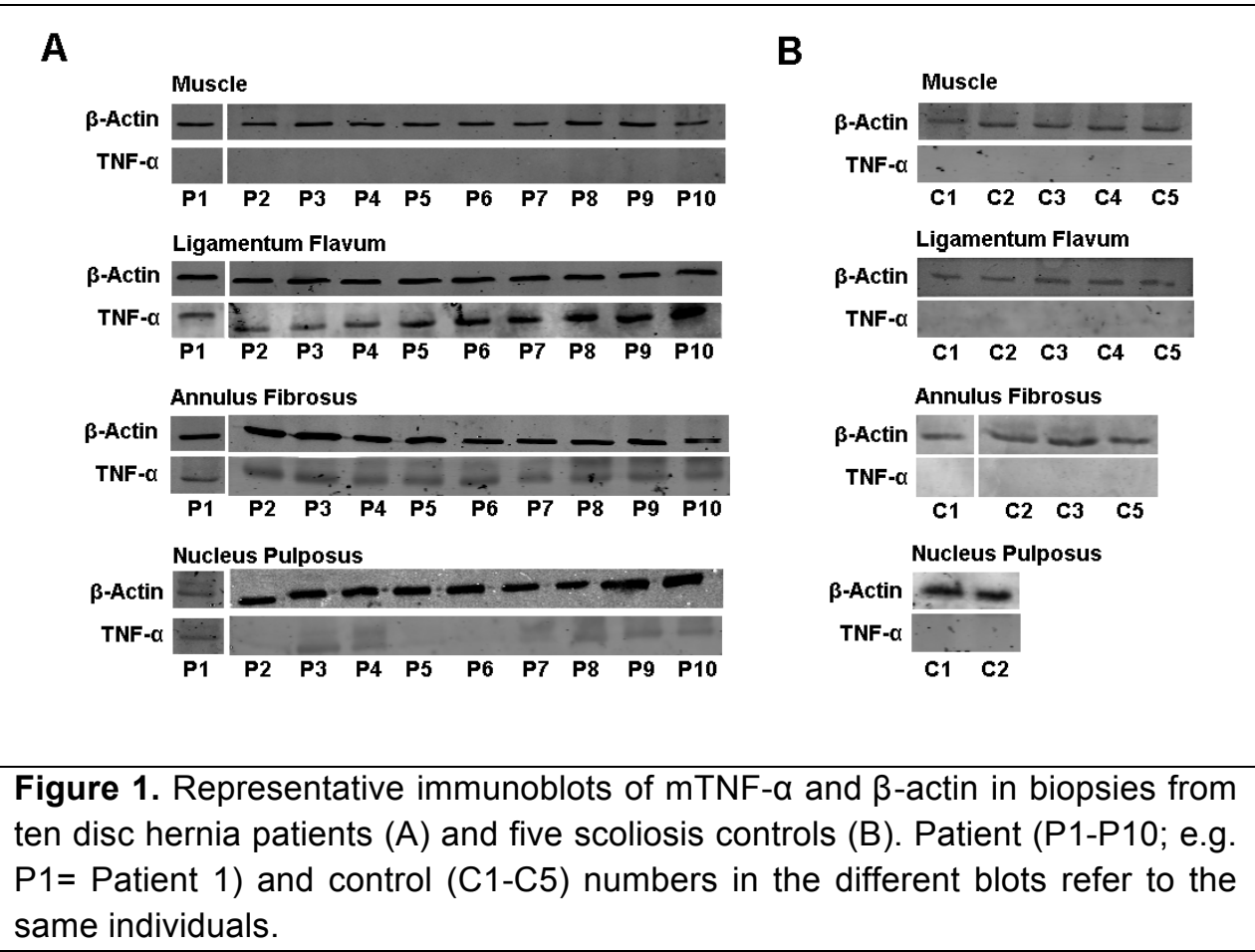

\subsection{TNF- $\alpha$ protein and mRNA levels in tissue biopsies}

Soluble TNF- $\alpha$ (sTNF- $\alpha$ ) results from proteolysis of membranous TNF- $\alpha$ (mTNF- $\alpha$ ) by TNF- $\alpha$ converting enzyme. Both, sTNF- $\alpha$ and mTNF- $\alpha$ can be detected by immunoblotting at 17 and $26 \mathrm{kDa}$, respectively. sTNF- $\alpha$ was not detected in controls and patients. mTNF- $\alpha$ was not detected in any tissue type from controls or in muscle biopsies from patients, but was observed in LF, AF, and NP of LDH patients (Figure 1). Differential cytokine expression was observed between patients throughout the diverse positive samples, especially in the NP. Optical density quantifications showed that the AF expressed the highest amount of TNF- $\alpha$ (Figure 2A). To investigate if pain sensation was related to a local inflammation in the disc, we analyzed the correlation between 
presurgical VAS scores and TNF- $\alpha$ expression in the three positive tissue types. Neither in LF, nor in AF or NP did TNF- $\alpha$ levels correlate with the presurgical VAS score. To investigate if a local inflammation in the disc would have a predictive value for the surgical outcome in terms of pain sensation, we analyzed the correlation between TNF- $\alpha$ expression in the three positive tissue types and VAS scores at 6 weeks and 12 months follow-up.

A

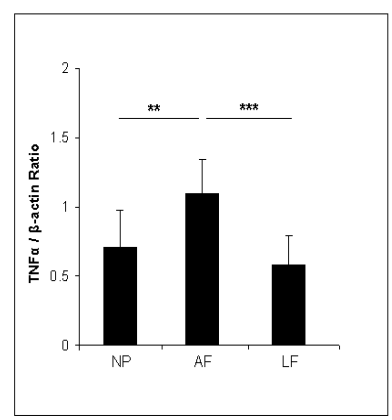

B
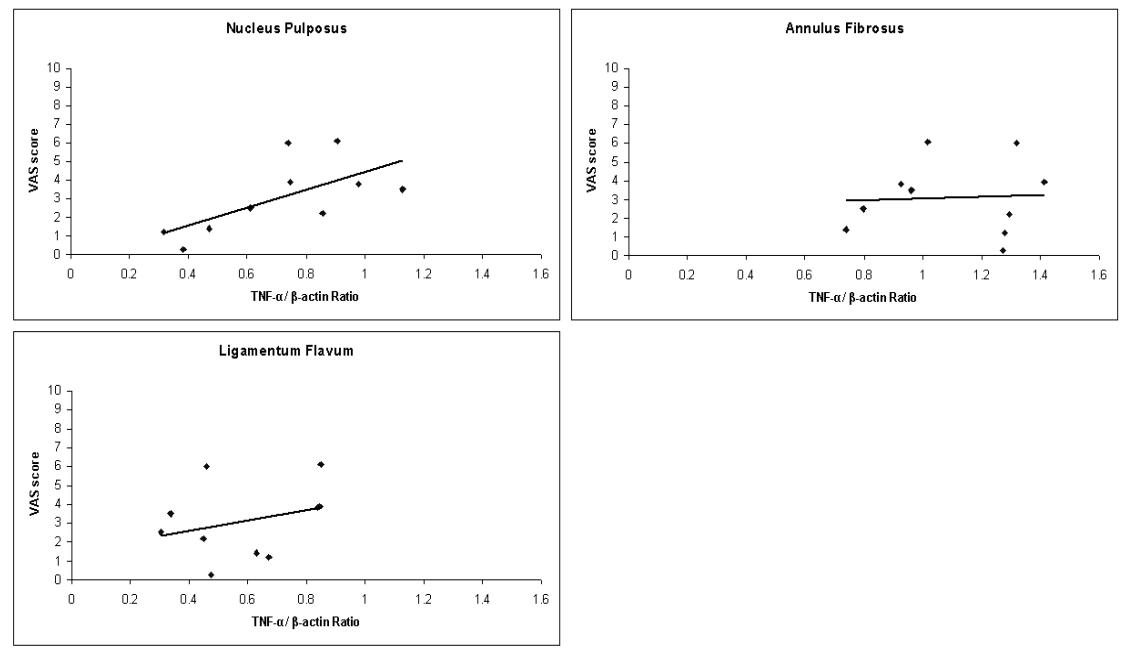

Figure 2. A: Optical density values of immunoblotted TNF- $\alpha / \beta$-actin. TNF- $\alpha$ was not detected in any tissue sample from controls or muscle biopsy from LDH patients, but was expressed in NP, AF and LF biopsies from LDH patients. Of these tissue types, the AF expressed the highest level of TNF- $\alpha$. Data are expressed as mean \pm SEM; ${ }^{* *} p<0.01 ;{ }^{* * *} p<0.001$. B: Correlation between VAS scores at 12 months follow-up and TNF- $\alpha$ expression in the NP, AF or LF. A positive correlation was found only for the NP $(r=0.65)$, and not for the AF 
$(\mathrm{r}=0.06)$ and $\mathrm{LF}(\mathrm{r}=0.29) . \mathrm{NP}=$ nucleus pulposus; $\mathrm{AF}=$ annulus fibrosus; $\mathrm{LF}=$ ligamentum flavum.

This analysis learned that TNF- $\alpha$ levels of the NP moderately correlated with VAS scores at 6 weeks $(r=0.54)$ and at 12 months $(r=0.65$; Figure 2B). No correlation was found between VAS scores at 6 weeks or 12 months and TNF- $\alpha$ expression in $L F(r=0.21$ and $r=0.29$, respectively) or $A F(r=0.05$ and $r=0.06$, respectively).

In order to corroborate the findings obtained through immunoblotting, qPCR was performed on the same samples. After normalizing samples to GAPDH expression, TNF- $\alpha$ mRNA was 2.5-4.5 fold increased in biopsies from LDH patients compared to those of controls (Figure 3). Similar to immunoblot data, TNF- $\alpha$ mRNA levels were highest in AF.

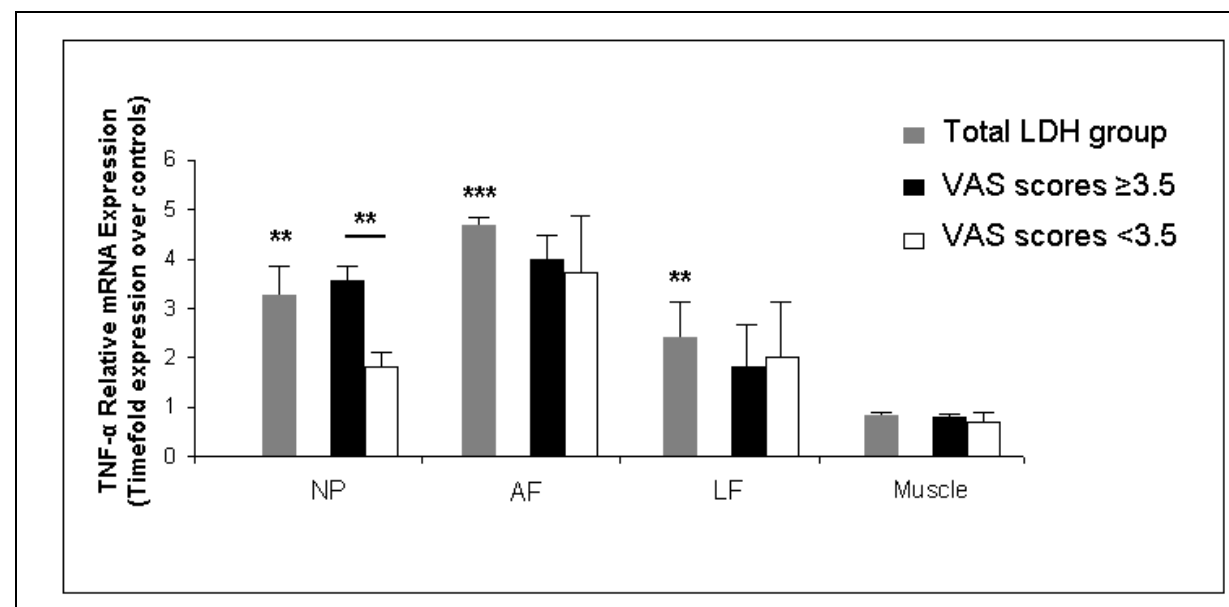

Figure 3. TNF- $\alpha$ mRNA expression in the total LDH patient group (grey bars), LDH patients with VAS scores $\geq 3.5$ (black bars), and LDH patients with VAS scores $<3.5$ (white bars) at 12 months follow-up. In the total LDH patient group, mRNA levels were significantly increased in the NP, AF, and LF, compared to the respective tissue types in controls. In the NP, patients with a VAS score $\geq 3.5$ expressed significantly more TNF- $\alpha$ mRNA than those with scores $<3.5$. $\mathrm{NP}=$ nucleus pulposus; $\mathrm{AF}=$ annulus fibrosus; $\mathrm{LF}=$ ligamentum flavum. Data are expressed as mean \pm SEM; ${ }^{* *} p<0.01,{ }^{* * *} p<0.001$. 
When patients were divided according to presurgical VAS scores at 6 weeks and 12 months into $a \geq 3.5$ and $a<3.5$ group, there was no difference in TNF- $\alpha$ mRNA expression for either tissue type. Also, in line with immunoblot data patients with VAS scores $\geq 3.5$ expressed significantly more TNF- $\alpha$ mRNA in the $\mathrm{NP}$, than those with scores $<3.5$. Again, this difference was found only for the NP and existed for VAS scores at 6 weeks and 12 months follow-up.

A

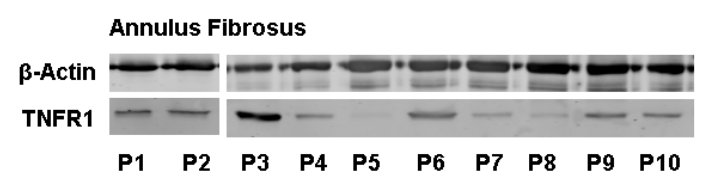

Nucleus Pulposus

$\beta$-Actin

TNFR1

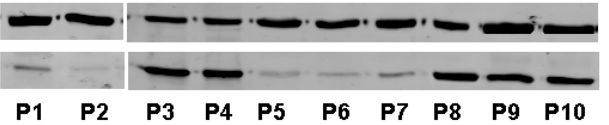

C

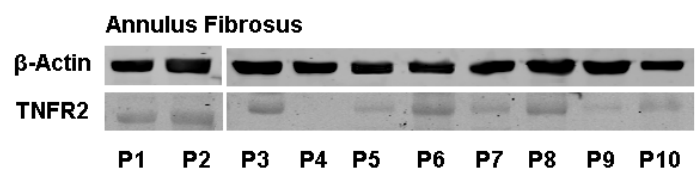

Nucleus Pulposus

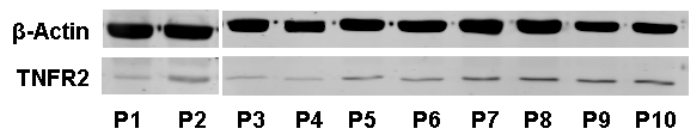

B

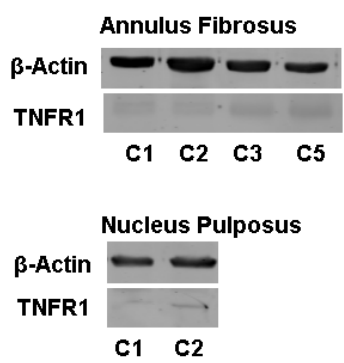

D
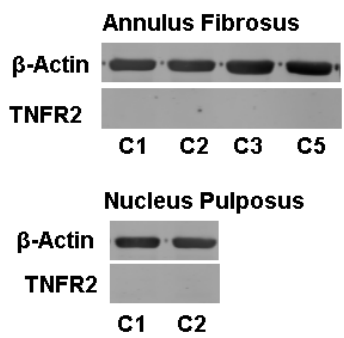

Figure 4. Representative immunoblots of TNFR1 from ten disc hernia patients $(A)$ and four scoliosis controls (B). Blots were made from AF and NP biopsies and included $\beta$-actin. The same tissue homogenates were blotted for TNFR2, i.e., patients $(C)$ and controls (D). Patient (P1-10) and control (C1-5) numbers in the different blots refer to the same individuals.

\subsection{TNFR1 and TNFR2 protein and mRNA levels in tissue biopsies}

By immunoblotting, TNFR1 was clearly detectable in AF and NP samples from LDH patients and hardly in samples from controls (Figure 4). Evident expression differences were present between patients. Quantification of 
immunoblots learned that TNFR1 was significantly more expressed in NP samples from patients than from controls, whereas in AF there was no difference between patients and controls (Figure 5). Whereas TNFR2 protein was not detectable in AF and NP biopsies from controls, it was observed in samples from the LDH patients group (Figures 4 and 5). To investigate if pain sensation was related to inflammation in the disc, we analyzed the correlation between VAS scores and TNFR expression. While no correlation was found between the VAS score at 1 day before surgery and the TNFR1 level, a higher TNFR1 protein expression in the NP correlated with more pain at 6 weeks $(r=0.75)$ and at 12 months ( $r=0.80$; Figure 5$)$. No correlation was found between TNFR1 expression in the AF and VAS scores at 6 weeks or 12 months followup ( $r=0.05$ and $r=0.02$, respectively). Also TNFR2 expression did not correlate with the VAS score at 1 day before surgery. However, a lower TNFR2 protein expression in the AF correlated with more pain at 6 weeks $(r=-0.67)$ and 12 months ( $r=-0.60$; Figure 5). No correlation was found between TNFR2 expression in the NP and VAS scores at 6 weeks or 12 months follow-up ( $r=-$ 0.18 and $r=-0.14$, respectively).

By qPCR, NP and AF samples from patients showed around 4-fold higher expression of TNFR1 and 1.5-fold increased levels of TNFR2 compared to that in samples from controls (Figure 6). When patients were grouped based on their VAS score at 6 weeks and 12 months follow-up, TNFR1 was increased and TNFR2 was decreased in NP samples from patients with a VAS score $\geq 3.5$ $(p<0.01)$. TNFR2 was also decreased in AF samples from patients with a VAS score $\geq 3.5(p<0.05)$.

\section{Discussion}

The association between proinflammatory cytokines and pain has been extensively reported in experimental models $[2,5,7,8,15,18,23,33,34,40$, 45]. In line with these observations, we were unable to detect TNF- $\alpha$ and its receptor in disc biopsies from scoliosis patients without pain, while biopsies from LDH patients with pain did show high levels of TNF- $\alpha$ and TNF- $\alpha$ receptors. 
Chapter 4

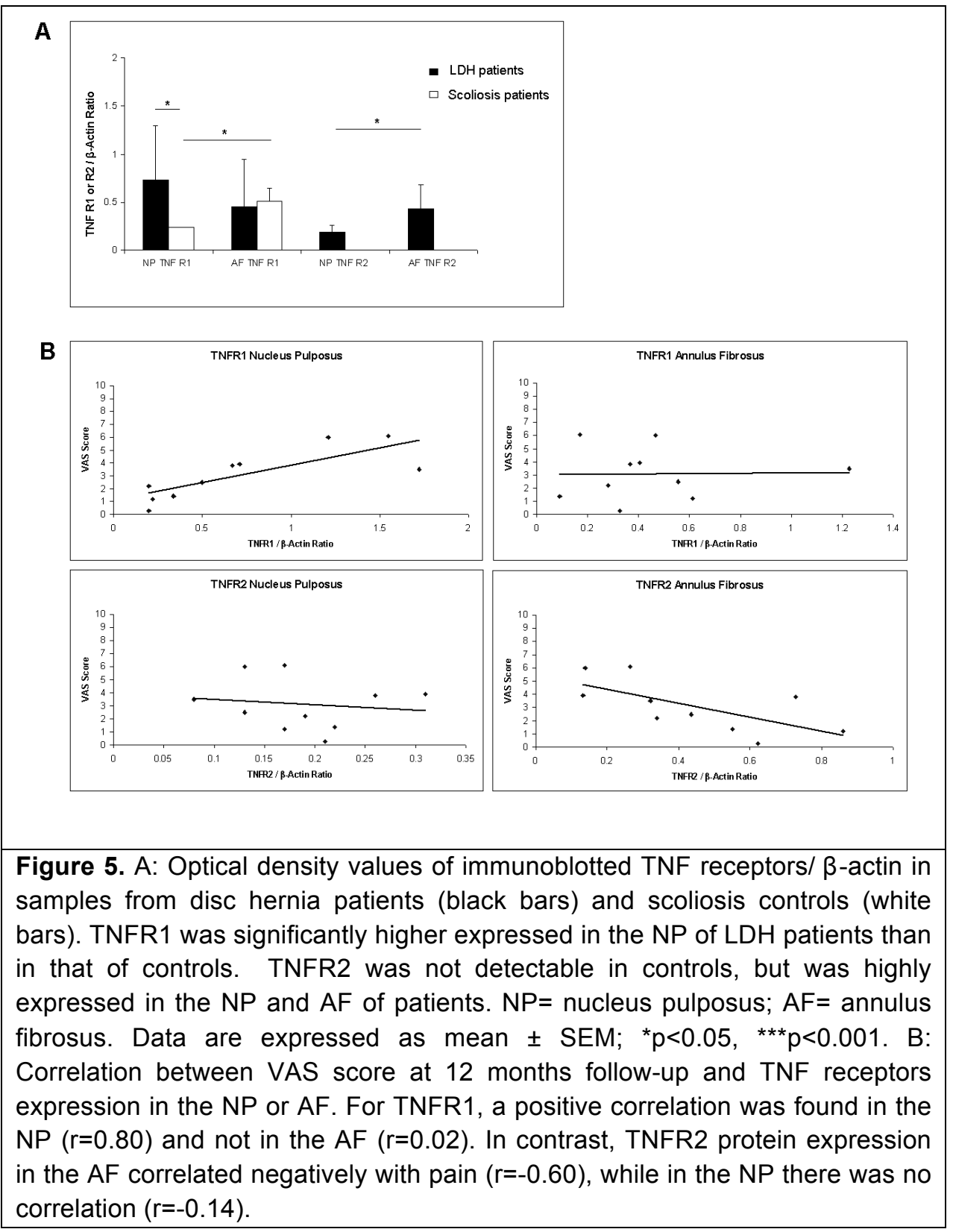

Remarkably, these levels did not correlate with presurgical pain sensation but did correlate with postoperative pain. This correlation was tissue specific, i.e., it 
existed for NP but not for AF or LF biopsies. Interestingly, an increased postoperative pain sensation was associated with increased TNFR1 and decreased TNFR2 levels.

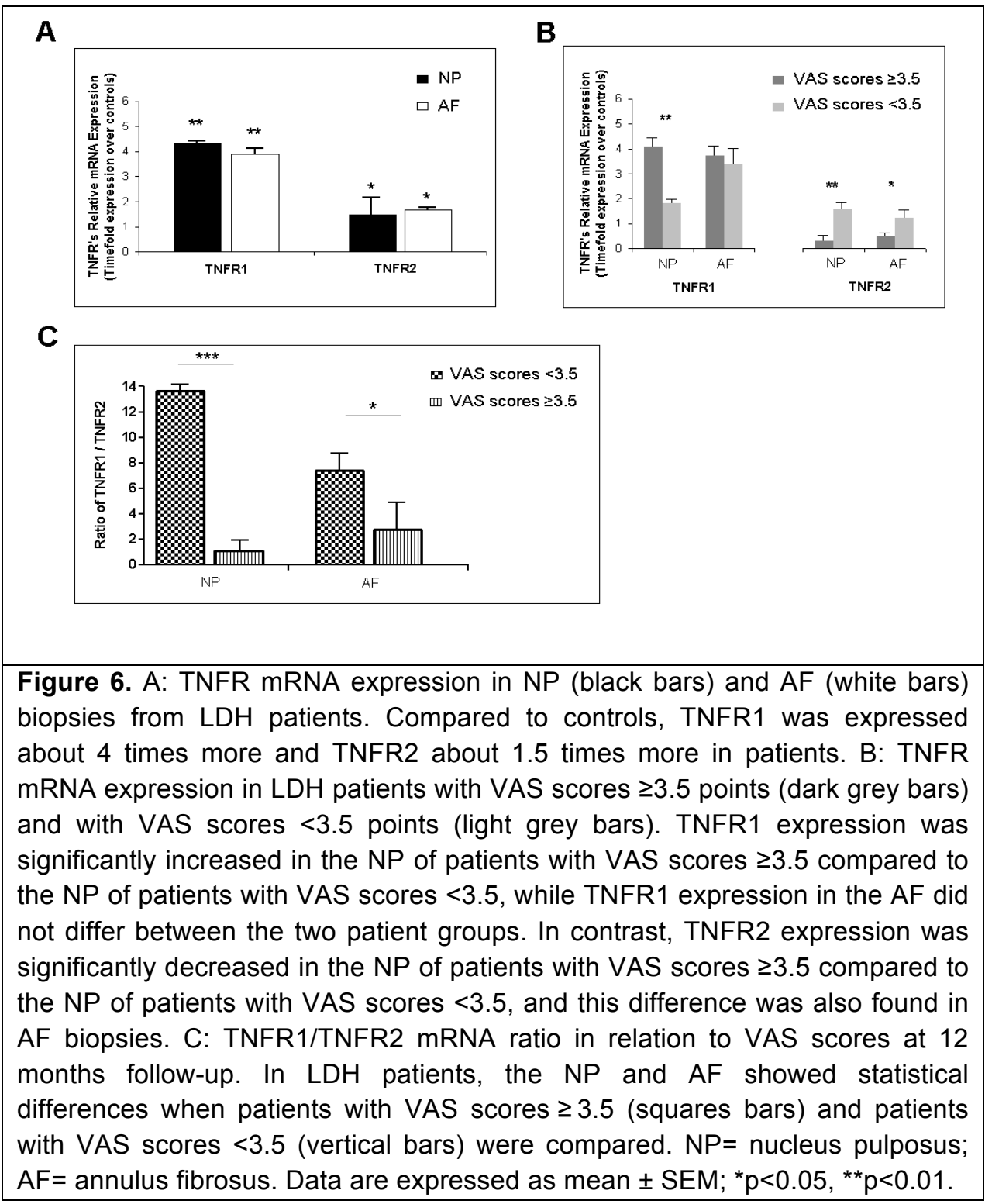


These findings are in line with the increased TNF- $\alpha$ serum levels reported in patients with various forms of chronic pain compared to painless patients and/or healthy controls. Populations associated with painful conditions showed significant peripheral presence of TNF- $\alpha$ and other cytokines in a diverse amount of neurological pathologies [24, 39]. However, the current data suggests that in LDH the inflammatory process responsible for pain is locally restricted to the NP and AF. In the abovementioned studies, which included heterogeneous neurological conditions, peripheral markers in blood were sufficient to correlate an inflammatory process with the intensity of pain. On the contrary, in our study we were unable to detect TNF- $\alpha$ in the most peripheral biopsies (paravertebral muscle). The observation that the NP is a potential source of TNF is in accordance with preclinical data [31, 32, 37]. These findings are in line with a previous study where peripheral blood samples were obtained systematically from a population of chronic low back pain patients and compared to healthy subjects, in order to determine the clinical relevance of TNF- $\alpha$ levels. This study reported no significant final correlation between the peripheral samples and pain [44]. Our findings corroborate previous findings from a study of complex regional pain syndrome patients where both, tissue biopsies and peripheral blood samples were collected, showing local but not systemic increase of TNF- $\alpha$ levels [25]. We showed that at the moment of surgery an inflammatory process is present in the LF, AF and NP. At this time point pain could be considered to be due to compression and thus inflammation surrounding the nerves. During the LDH surgery the cause of compression and a large part of the inflamed tissues were removed. Interestingly, postoperative pain correlated to increased TNF- $\alpha$ and TNFR1 and decreased TNFR2 levels in intraoperatively collected disc tissue. This may indicate that either an inflammatory process originated from nerve endings or surrounding tissue which is not removed during the surgery is perpetuated or that different molecules take the lead of the pain sensation. In this respect, a recent preclinical study showed that the inflammatory response was resolved soon after the insult, and that it was followed by expression of inflammatory neuropeptides in the dorsal root ganglion in a permanent manner [30]. In addition, a study performed in rats showed that the application of a TNF- $\alpha$ inhibitor (etanercept) following disc puncture down regulated the expression of calcitonin gene-related peptide in the dorsal root ganglions responsible for the intervertebral discs innervations, demonstrating that neuropeptides action could be diminished if the early inflammatory process is properly contained [23]. Apparently, we could speculate that an early vicious circle created by TNF- $\alpha$ producing edema, apoptosis or necrosis becomes eventually perpetuated by 
different players. In our data, TNF- $\alpha$ expression did not correlate with VAS scores at 1 day before surgery. This may relate to the observation that all patients experienced high levels of pain and as a consequence displayed similar VAS scores 1 day before surgery. Therefore it was not possible to divide patients in two clear opposite groups. In contrast, at 6 weeks and 12 months follow-up the symptomatology divided the patients into two distinct groups. These observations do not necessarily imply the perpetuation of the inflammatory process orchestrated by TNF- $\alpha$ and its receptors; because presumably a larger portion of inflammation would be resolved once the herniation is removed. However, as it has been demonstrated in preclinical studies, the permanent damage produced by the inflammatory process could be responsible for the chronic symptomatology [30]. This suggests that an initial elevated inflammatory response is directly correlated with the tissue damage and therefore with the long-term outcome.

With respect to TNF- $\alpha$ receptors, our data suggest that the intensity of pain is regulated in an opposite manner between TNFR1 and TNFR2; the former being related to elevated pain scores and the latter to a lower intensity or absence of pain. These data could correspond to the primary effect associated to each receptor where up-regulation of TNFR1 leads to apoptosis and the upregulation of TNFR2 results in cell survival and proliferation $[1,11,12,14,28$, 38]. The cellular damage produced during the inflammatory process could dictate the degree of sensitization that later on will determine the permanent clinical outcome. Why the presence of TNF- $\alpha$ up-regulates more TNFR1 or TNFR2 differently in patients is still to be clarified. It would also be important to establish temporal expression differences of these receptors and the association of the amount of damage produced when comparing acute and chronic conditions. Probably, receptor expression will be affected according to the quantity of apoptosis produced as a consequence of inflammation throughout days and weeks. So far, we could infer that these receptors work as a balance, where depending on the level of expression it could generate two opposite clinical outcomes. However, intracellular crosstalks between the two receptors complicate the prediction in case of abnormal cell signaling. Here, we show that the ratio signature of TNFR1/TNFR2 mRNA is increased with an increasing VAS score at 12 months. These data confirmed that patients with higher pain scoring express elevated levels of TNFR1 over TNFR2; and on the contrary, that in those patients with low pain scores, this ratio is significantly diminished in the final outcome. In this respect, a recent in vivo and in vitro study concluded that TNFR1 is responsible for moderate neuronal excitation and pain behavior in uninjured neural tissue, whereas TNFR2 can only act 
together with activated TNFR1 [33]. Intrathecal injection of TNFR1 and particularly the combination of TNFR1 and TNFR2 elicited pain responses in rats, but injection of TNFR2 did not produced pain responses. Moreover, naïve dorsal root ganglion single-fiber recordings showed that only TNFR1 stimulation could induce spontaneous activity. Consequently, their findings imply that TNFR2 excitatory actions can only occur once a lesion is established in the nerve. Similarly, in our study TNFR1 was present in patients and controls, whereas TNFR2 was only present in LDH patients. Also, in accordance with other studies describing TNFR1 as constitutively expressed in various tissue types and that TNFR2 requires to be induced by an inflammatory response [20, $21,41]$. As recently shown in mice with an inflammatory pain model, another potential difference between receptors could be related to temporal expression and mediation of pain. In this study, the authors propose that early inflammation is regulated by TNFR2 [46].

It is important to remark that in our study only mTNF- $\alpha$ was found and that in any case sTNF- $\alpha$ was detected. This is significant due to the fact that mTNF- $\alpha$ is able to interact with both receptors, in comparison to sTNF- $\alpha$, which has been described to interact primarily with TNFR1 [16, 17, 19]. These findings may have therapeutic implications because certain inhibitors are designed only to block sTNF- $\alpha$. In addition, recent findings suggest that microglial communication may be performed throughout its membrane-bound TNF- $\alpha$ component, because this molecule works as a ligand and a receptor facilitating messaging between cells $[9,22,47]$. On the same way, knowing if a patient has developed higher expression levels for either one receptor or the other could help us predict the pain related outcome after discectomy. In the future, these insights may add to the selection criteria for surgery. The cellular mechanisms behind this shift in TNFR1/TNFR2 are not fully elucidated, but may prove to be a potential target for therapy. A valuable contribution in this area may come from in vitro studies on intervertebral disc biopsies. In a recent in vitro study, the application of a soluble TNFR2 antagonized the inflammatory action produced by TNF- $\alpha$ in human discs cells [35], demonstrating a possible application of this type of therapy.

Our findings altogether provide evidence that inflammatory profiles could predict the outcome after surgery in LDH patients. In that case, local anti-inflammatory treatments focusing on mTNF- $\alpha$ and its receptors could help modulate the amount of pain, such as adalimumab, etanercept or infliximab [11, 13, 27, 35]. Even though the mechanisms responsible for chronic pain are poorly understood, this study might contribute to prediction of the clinical outcome after LDH surgery and to design novel therapeutic approaches. 
TNF-alpha levels correlate with postoperative pain in LDH patients

\section{References}

1. Aggarwal BB. Signalling pathways of the TNF superfamily: a doubleedged sword. Nat Rev Immunol 2003;3:745-756.

2. Bianchi $M$, et al. Increased tumor necrosis factor-alpha and prostaglandin E2 concentrations in the cerebrospinal fluid of rats with inflammatory hyperalgesia: the effects of analgesic drugs. Anesth Analg 2007;104:949-954.

3. Carragee EJ, et al. Activity restrictions after posterior lumbar discectomy. A prospective study of outcomes in 152 cases with no postoperative restrictions. Spine 1999;24:2346-2351.

4. Carragee EJ, et al. A prospective controlled study of limited versus subtotal posterior discectomy: short-term outcomes in patients with herniated lumbar intervertebral discs and large posterior anular defect. Spine 2006;31:653-657.

5. Covey WC, et al. Expression of neuron-associated tumor necrosis factor alpha in the brain is increased during persistent pain. Reg Anesth Pain Med 2002;27:357-366.

6. Croft PR, et al. Outcome of low back pain in general practice: a prospective study. BMJ 1998;316:1356-1359.

7. DeLeo JA, et al. Cytokine and growth factor immunohistochemical spinal profiles in two animal models of mononeuropathy. Brain Res 1997;759:50-57.

8. DeLeo JA, et al. Transgenic expression of TNF by astrocytes increases mechanical allodynia in a mouse neuropathy model. Neuroreport 2000;11:599-602.

9. Eissner $\mathrm{G}$, et al. Ligands working as receptors: reverse signaling by members of the TNF superfamily enhance the plasticity of the immune system. Cytokine Growth Factor Rev 2004;15:353-366.

10. Elliott $A M$, et al. The epidemiology of chronic pain in the community. Lancet 1999;354:1248-1252.

11. Faustman D, Davis M. TNF receptor 2 pathway: drug target for autoimmune diseases. Nat Rev Drug Discov 2010;9:482-493.

12. Fontaine $\mathrm{V}$, et al. Neurodegenerative and neuroprotective effects of tumor Necrosis factor (TNF) in retinal ischemia: opposite roles of TNF receptor 1 and TNF receptor 2. J Neurosci 2002;22:RC216.

13. Genevay $S$, et al. Adalimumab in severe and acute sciatica: a multicenter, randomized, double-blind, placebo-controlled trial. Arthritis Rheum 2010;62:2339-46. 
14. George A, et al. Tumor necrosis factor receptor 1 and 2 proteins are differentially regulated during Wallerian degeneration of mouse sciatic nerve. Exp Neurol 2005;192:163-166.

15. George A, et al. Serial determination of tumor necrosis factor-alpha content in rat sciatic nerve after chronic constriction injury. Exp Neurol 1999;160:124-132.

16. Grell $\mathrm{M}$, et al. The transmembrane form of tumor necrosis factor is the prime activating ligand of the $80 \mathrm{kDa}$ tumor necrosis factor receptor. Cell 1995;83:793-802.

17. Grell M, et al. Induction of cell death by tumour necrosis factor (TNF) receptor 2, CD40 and CD30: a role for TNF-R1 activation by endogenous membrane-anchored TNF. EMBO J 1999;18:3034-3043.

18. Hatashita $S$, et al. Contralateral neuropathic pain and neuropathology in dorsal root ganglion and spinal cord following hemilateral nerve injury in rats. Spine 2008;33:1344-1351.

19. Herrera PL, et al. A mouse CD8 T cell-mediated acute autoimmune diabetes independent of the perforin and Fas cytotoxic pathways: possible role of membrane TNF. Proc Natl Acad Sci U S A 2000;97:279-284.

20. Holmes GM, et al. Immunocytochemical localization of TNF type 1 and type 2 receptors in the rat spinal cord. Brain Res 2004;1025:210-219.

21. Holtmann $\mathrm{MH}$, et al. The emerging distinct role of TNF-receptor 2 (p80) signaling in chronic inflammatory disorders. Arch Immunol Ther Exp (Warsz) 2002;50:279-288.

22. Horiuchi T, et al. Transmembrane TNF-alpha: structure, function and interaction with anti-TNF agents. Rheumatology 2010;49:1215-1228.

23. Horii $M$, et al. Direct application of the tumor necrosis factor- $\alpha$ inhibitor, etanercept, into a punctured intervertebral disc decreases calcitonin gene-related peptide expression in rat dorsal root ganglion neurons. Spine 2011;36:E80-5.

24. Koch A, et al. Nitric oxide and pro-inflammatory cytokines correlate with pain intensity in chronic pain patients. Inflamm Res 2007;56:32-37.

25. Krämer HH, et al. TNF-alpha in CRPS and 'normal' trauma - Significant differences between tissue and serum. Pain 2010; 152:285-90.

26. Locksley RM, et al. The TNF and TNF receptor superfamilies: integrating mammalian biology. Cell 2001;104:487-501.

27. Marchand $F$, et al. Effects of Etanercept and Minocycline in a rat model of spinal cord injury. Eur J Pain 2009;13:673-81. 
28. Marchetti L, et al. Tumor necrosis factor (TNF)-mediated neuroprotection against glutamate-induced excitotoxicity is enhanced by $\mathrm{N}$-methyl-D-aspartate receptor activation. Essential role of a TNF receptor 2-mediated phosphatidylinositol 3-kinase-dependent NF-kappa B pathway. J Biol Chem 2004;279:32869-32881.

29. Mariconda M, et al. Minimum 25-year outcome and functional assessment of lumbar discectomy. Spine 2006;31:2593-2599.

30. Miyagi $M$, et al. Disk Injury in Rats Produces Persistent Increases in Pain-Related Neuropeptides in Dorsal Root Ganglia and Spinal Cord Glia But Only Transient Increases in Inflammatory Mediators: Pathomechanism of Chronic Diskogenic Low Back Pain. Spine 2011;36:2260-6.

31. Murata $Y$, et al. The role of tumor necrosis factor-alpha in apoptosis of dorsal root ganglion cells induced by herniated nucleus pulposus in rats. Spine 2008;33:155-162.

32. Murata $Y$, et al. Selective inhibition of tumor necrosis factor-alpha prevents nucleus pulposus-induced histologic changes in the dorsal root ganglion. Spine 2004;29:2477-2484.

33. Schäfers $M$, et al. Selective stimulation of either tumor necrosis factor receptor differentially induces pain behavior in vivo and ectopic activity in sensory neurons in vitro. Neuroscience 2008;157:414-423.

34. Schäfers $M$, et al. Intramuscular injection of tumor necrosis factor-alpha induces muscle hyperalgesia in rats. Pain 2003;104:579-588.

35. Sinclair SM, et al. Attenuation of Inflammatory Events in Human Intervertebral Disc Cells with a Tumor Necrosis Factor Antagonist. Spine 2011;36:1190-6.

36. Sorkin LS, et al. Tumour necrosis factor-alpha induces ectopic activity in nociceptive primary afferent fibres. Neuroscience 1997;81:255-262.

37. Studer R, et al. Human Nucleus Pulposus Cells React to IL-6: independent actions and amplification of response to IL-1 and TNF- $\alpha$. Spine 2010;36:593-9.

38. Tartaglia LA, et al. A novel domain within the $55 \mathrm{kd}$ TNF receptor signals cell death. Cell 1993;74:845-853.

39. Üçeyler $\mathrm{N}$, et al. Differential expression of cytokines in painful and painless neuropathies. Neurology 2007;69:42-49.

40. Üçeyler N, et al. Early cytokine gene expression in mouse CNS after peripheral nerve lesion. Neurosci Lett 2008;436:259-264.

41. Vandenabeele $P$, et al. Two tumour necrosis factor receptors: structure and function. Trends Cell Biol 1995;5:392-399. 
Chapter 4

42. Wagner R, Myers RR. Endoneurial injection of TNF-alpha produces neuropathic pain behaviors. Neuroreport 1996;7:2897-2901.

43. Wajant $\mathrm{H}$, et al. Tumor necrosis factor signaling. Cell Death Differ 2003;10:45-65.

44. Wang $\mathrm{H}$, et al. The role of TNF-alpha in patients with chronic low back pain-a prospective comparative longitudinal study. Clin J Pain 2008;24:273-278.

45. Xu JT, et al. The role of tumor necrosis factor-alpha in the neuropathic pain induced by Lumbar 5 ventral root transection in rat. Pain 2006;123:306-321.

46. Zhang L, et al. TNF-alpha contributes to spinal cord synaptic plasticity and inflammatory pain: Distinct role of TNF receptor subtypes 1 and 2 . Pain 2011;152:419-27.

47. Zhou Z, et al. A novel cell-cell signaling by microglial transmembrane TNFa with implications for neuropathic pain. Pain 2010;151:296-306. 


\section{Elevated IL-1beta and IL-6 levels in lumbar herniated discs in patients with sciatic pain}

Pablo Andrade, Govert Hoogland,

Miguel A. Garcia, Harry W.M. Steinbusch, Marc A. Daemen, Veerle Visser-Vandewalle

Revision submitted to European Spine Journal 


\section{Chapter 5}

\section{Abstract}

Previous experimental models have shown that proinflammatory cytokines modulate peripheral and central nociception. However, the direct correlation between inflammation and pain in patients remains unclear. Our aim is to correlate the level of inflammation in the spine with pre- and postoperative pain scores after discectomy. Paravertebral muscle, annulus fibrosus (AF) and nucleus pulposus (NP) biopsies were intraoperatively collected from ten lumbar disc hernia (LDH) patients suffering from chronic sciatic pain and, as painless controls, five scoliosis patients. IL-1 $\beta$ and IL- 6 expression in these biopsies was assessed by qPCR and Western blot. The amount of pain, indicated on a 0 10point visual analogue scale (VAS), was assessed 1 day before surgery and 6 weeks and 1 year after surgery. For analysis purposes, LDH patients were grouped in painful (VAS $\geq 3.5$ ) and nonpainful (VAS<3.5). LDH painful patients group showed a 1-fold increased mRNA expression of IL-1 $\beta$ in the NP, and IL-6 in the AF and NP ( $p<0.05$ vs. controls). By Western blot analysis, both cytokines were clearly visible in all LDH biopsies, but not in controls. However, cytokine expression of the painful patients group did not differ from those of the nonpainful patients group. In addition, there was no correlation between VAS scores and either marker. These findings support the idea that LDH is accompanied by a local inflammatory process. Yet, the lack of correlation between IL-1 $\beta$ or IL- 6 expression and the severity pain suggests that these cytokines may not play a leading role in maintaining a pain generating network. 


\section{Introduction}

The primary functions of the intervertebral disc include shock absorption, stabilization, movement and flexibility of the spine. These tasks are possible due to the proper composition and interaction of its two main structures, the hard external ring named annulus fibrosus (AF) and the soft core of the disc named nucleus pulposus (NP). When one of these structures, or both, bulges or migrates out of the normal intervertebral space delimitation, a herniated disc occurs. If a herniated disc develops, the protrusion from the disc could compress one of the nerve roots or the spinal cord itself. The compression of neural tissue produced by herniated disc material may generate diverse clinical symptoms, among which pain is one of the most common presentations. The symptomatology that results from lumbar disc hernia (LDH), which produces a painful sensation radiating into the leg, is known as sciatica $[1,2]$. The modalities to treat sciatic pain may include non-surgical treatment (physiotherapy and medication) and surgery. The general consensus is that conservative therapy should be followed during the first 6 to 8 weeks from the initiation of symptoms, unless absolute surgical indications are present (increasing muscle weakness, alterations of the urinary bladder function or opioid-resistant pain) [3, 4]. This non-surgical treatment results in recovery in about $80 \%$ of the cases [4]. For the remaining patients the option of disc surgery is considered to be an acceptable treatment alternative, though the outcome after 1 year is comparable to that of non-surgical therapy [1]. This suggests that mechanisms other than pure mechanical compression may underlie the pathophysiology of sciatic pain in LDH.

It is generally recognized that neuroinflammation plays a crucial role in the generation of chronic pain. Numerous in vitro and animal models of pain have demonstrated the contribution of proinflammatory cytokines to peripheral and central nociception [5-10]. Also in human, local and systemically expressed proinflammatory cytokines have been implicated as mediators of pain [11-13]. Among these, TNF- $\alpha, \mathrm{IL}-1 \beta$ and IL- 6 are particularly interesting because of their hyperalgesic effects after nerve damage. However, while the contribution of TNF- $\alpha$ to chronic pain has been shown repetitively, data on the roles of IL-1 $\beta$ and IL-6 in human sciatic pain are scarce. This study assessed if local IL-1 $\beta$ and IL-6 levels in LDH patients related to presurgical pain and to the outcome of discectomy. 


\section{Chapter 5}

\section{Methods}

\subsection{Patients and pain evaluation}

This study was approved by the Ethics Committee at Maastricht University Medical Center. Fifteen patients were prospectively enrolled at the departments of neurosurgery and orthopedics, and included ten LDH patients suffering from leg pain for more than 1 year and five scoliosis patients without painful symptomatology or degenerative disc disease. The LDH patient group had a mean age of 41 years and consisted of 5 males and 5 females. The scoliosis patient group had a mean age of 21 years and consisted of 3 males and 2 females. LDH patients assessed the intensity of sciatic pain on a 0-10 ( $0=$ no pain; 10=worst pain) visual analogue scale (VAS) at 1 day before discectomy, and at 6 weeks and 1 year after surgery. The duration of symptoms before the surgical procedure in LDH patients included in this study were greater than three months and less than six months in all cases. General clinical data were recorded prospectively and preoperative MRI was performed in all cases to corroborate the clinical examination findings. All patients in the LDH group $(n=10 / 10)$ were treated with non-steroidal anti-inflammatory drugs during the period previous to the surgery. Patients were suspended anti-inflammatory medication for 7 days before surgery. All patients underwent elective spinal surgery for the first time. Intraoperatively collected biopsies, obtained during discectomy in LDH patients and during corrective orthopedic surgery in scoliosis patients, were collected from the paravertebral muscle $(n=15)$, the AF $(n=14)$ and the NP $(n=12)$. Immediately upon collection, they were divided in two equal parts (one for qPCR and one for Western blot) frozen in liquid nitrogen, and stored at $-80^{\circ} \mathrm{C}$. LDH patients were divided in two groups according to their postoperative pain scores.

\section{2. Quantitative polymerase chain reaction}

Total RNA extraction was performed using TRIzol (Invitrogen, CA), according to the manufacturer's protocol. RNA purity and concentration were measured by optical density, using a NanoDrop ND-1000 spectrophotometer (Thermo Fisher Scientific, Waltham, USA). Next, $1 \mu \mathrm{g}$ RNA was first incubated for $10 \mathrm{~min}$ at $70^{\circ} \mathrm{C}$ in order to prevent secondary structures and then reverse transcribed using a First Strand cDNA Synthesis Kit (Fermentas International Inc., Canada). Gene expression analysis was performed in optical 96-well plates using a LightCycler 480 Real-Time PCR System. PCR conditions consisted of a $20 \mu \mathrm{l}$ 
reaction volume, containing 10 $\mu$ S SYBR Green I Master (Roche Diagnostics, IN), $0.2 \mu \mathrm{l}$ forward and reverse primer, $4.8 \mu \mathrm{l}$ water and 250ng cDNA in a volume of $5 \mu \mathrm{l}$. Amplicons were generated using the following primer sets: IL-1 $1 \beta$-sense (5'-TTG ACG GAC CCC AAA AGA TG-3') and antisense (5'-AGA AGG TGC TCA TGT CCT CA-3'), and IL-6-sense (5'-GTT CTC TGG GAA ATC GTG GA$\left.3^{\prime}\right)$ and antisense (5'-TGT ACT CCA GGT AGC TAT GG-3'). Glyceraldehyde 3phosphate dehydrogenase (GAPDH) was used as the housekeeping gene to normalize the samples; sense (5'-ACA GTC AGC CGC ATC TTC-3') and antisense (5'-CCA ATA CGA CCA AAT CCG TTG-3'). The conditions for the reaction included 50 cycles in a fixed sequence $94^{\circ} \mathrm{C}$ for $30 \mathrm{~s}, 63^{\circ} \mathrm{C}$ for $15 \mathrm{~s}$, and $72^{\circ} \mathrm{C}$ for $15 \mathrm{~s}$. At the end of the PCR, a general dissociation curve was made and amplicons were resolved on a $2 \%$ agarose gel to assess product specificity.

\section{3. Western Blot analysis}

Biopsies were homogenized in lysis buffer containing $0.01 \mathrm{M}$ phosphate buffered saline (PBS), 1\% Igepal, 1mM EDTA, 1mM EGTA, 0.1\% Triton and COMPLETE protease inhibitor cocktail (Roche Diagnostics; $1 \mathrm{~g}$ weight tissue in

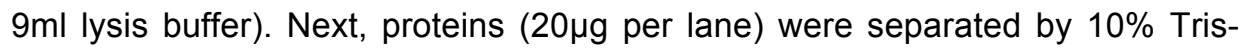
$\mathrm{HCl}$ SDS-polyacrylamide gel electrophoresis and then transferred to a pure nitrocellulose membrane for a period of 60 minutes at $100 \mathrm{~V}$ using a Bio-Rad Laboratories system. After transfer, the membranes were rinsed 1 time with PBS and then blocked for 60 minutes in 1:2 blocking buffer: PBS (Odyssey blocking buffer. LI-COR Biosciences, NE). Next, membranes were incubated overnight at $4^{\circ} \mathrm{C}$ with either 1:500 diluted monoclonal mouse anti-human IL-1 $\beta$ (clone 5C10, Santa Cruz Biotechnology, CA) and 1:500 diluted monoclonal mouse anti-human $\beta$-Actin (clone ACTBD11B7, Santa Cruz Biotechnology) or with 1:500 diluted polyclonal rabbit anti-human IL-6 (clone C-20, Santa Cruz Biotechnology) and 1:500 diluted monoclonal mouse anti-human $\beta$-Actin (clone ACTBD11B7, Santa Cruz Biotechnology). Following primary antibody incubation, membranes were rinsed during 3 cycles with PBS/PBS+Tween/PBS and then incubated 60 minutes at room temperature with their corresponding secondary antibody, i.e., Alexa Fluor 680 donkey anti-mouse lgG or Alexa Fluor 800 goat anti-rabbit IgG (1:10.000; Molecular Probes, OR). All antibodies were diluted in 1:2 blocking buffer:PBS. Finally, immunoreactive protein bands were visualized by enhanced chemiluminescence detection using a Odyssey Infrared Imaging System (LI-COR Biosciences), and quantified by calculating the optical 
Chapter 5

density ratio of the $17 \mathrm{kDa} \mathrm{IL}-1 \beta$ or $80 \mathrm{kDa} \mathrm{IL}-6$ band and the $43 \mathrm{kDa} \beta$-Actin band using a digital imaging analysis system (Image J 1.42q NIH, USA).

\section{4. Statistical analysis}

A Kruskal-Wallis test was applied to statistically test differences in protein expression between painful and non-painful LDH patient groups and the scoliosis control group, followed by Dunn post hoc tests. The $p$-value was considered significant with an alpha $<0.05$. Linear regression analysis was used to measure the correlation between VAS score and IL-1 $\beta$ or IL-6 protein expression.
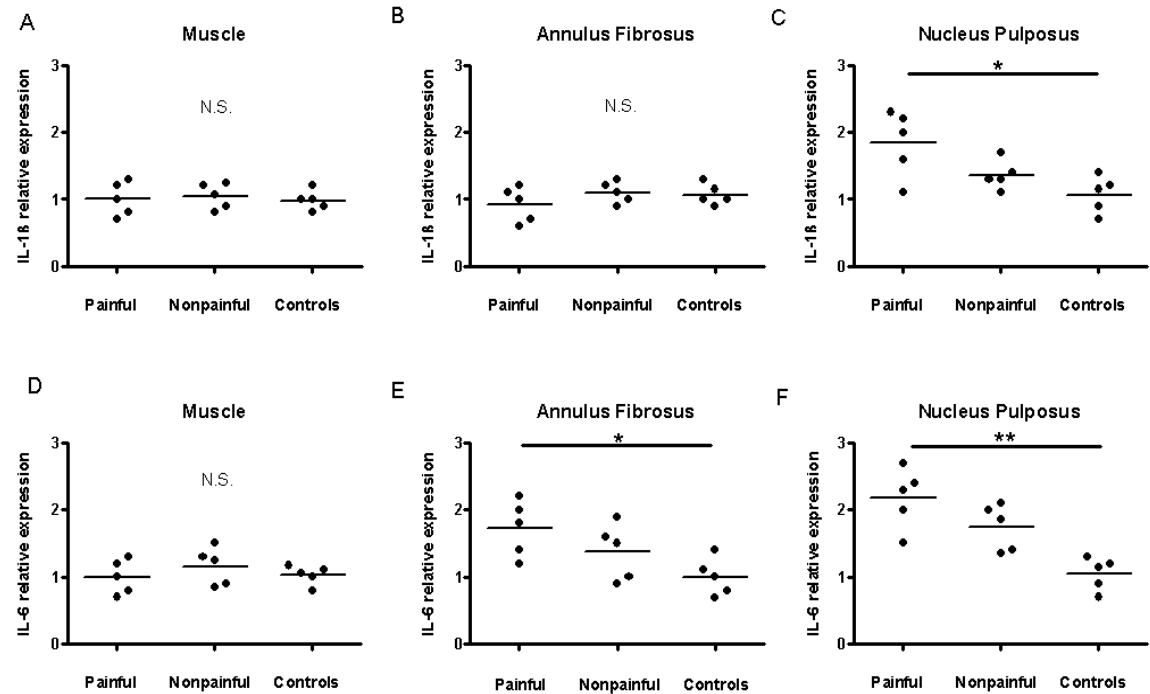

Figure 1. Distribution of individual IL-1 $\beta(A-C)$ and IL-6 (D-F) mRNA levels in muscle $(A, D)$, annulus fibrosus $(B, E)$ and nucleus pulposus $(C, F)$ samples from LDH patients and scoliosis controls $(n=5)$. Based on 1 year follow-up VAS scores, the LDH patient group was divided in a painful (VAS $\geq 3.5 ; n=5)$ and a non-painful (VAS<3.5; $n=5$ ) outcome group. Expression levels were normalized to GAPDH as reference gene and are represented as time-fold increase over controls. N.S. $=$ Non-significant. ${ }^{*} p<0.05$ and ${ }^{* *} p<0.01$. 


\section{Results}

\subsection{VAS scores}

Average \pm SD VAS scores were $6.8 \pm 1.2$ ( 1 day before surgery), $3.0 \pm 1.9$ (6 weeks after surgery), and $3.9 \pm 2.4$ (1 year after surgery). Average $\pm S D$ delta VAS scores compared to presurgical evaluation were $3.8 \pm 1.9$ (6 weeks after surgery), and 3.0 \pm 2.5 (1 year after surgery). Based on the 1-year follow-up data, the LDH patient group was divided in a painful (VAS $\geq 3.5 ; n=5 ; 2$ females and 3 males) and a non-painful (VAS $<3.5 ; n=5 ; 3$ females and 2 males) outcome group. This division was performed since a score of 4 points is described as a disruption for the quality of life.

3. 2. LDH patients show a localized increase in IL-1 $1 \beta$ and IL-6 mRNA expression

IL-1 $\beta$ and IL-6 gene expression in paravertebral muscle samples from LDH patients was comparable to that from scoliosis controls (Figures 1A and 1D). IL$1 \beta$ gene expression in AF samples from LDH patients was also comparable to that from scoliosis controls (Figure 1B). Yet, the IL- 6 gene was about 0.8 times more expressed in AF samples from painful LDH patients than in that from controls (Figure 1E). Also, NP samples of LDH patients with a painful outcome showed a 1 time higher IL-1 $\beta$ and IL- 6 mRNA expression than controls (Figures $1 \mathrm{C}$ and $1 \mathrm{~F})$. In neither tissue sample did IL-1 $\beta$ or IL-6 expression levels of the non-painful patient group differ from that of the painful patient group or from controls 1 day before surgery, or 6 weeks and 1 year after surgery.

\section{3. IL-1 $1 \beta$ and IL-6 protein is expressed in LDH samples but not in controls}

Immunoblotting showed no detectable IL-1 $\beta$ or IL- 6 in control samples, whereas both proteins were clearly visible in all LDH samples. However, IL-1 $\beta$ and IL-6 protein expression varied between the different tissue types, with the lowest expression in muscle and the highest in NP samples (Figure 2). There was no difference in IL-1 $\beta$ or IL- 6 protein level between the painful and the non-painful group in either tissue type 1 day before surgery, or 6 weeks and 1 year after surgery. On the contrary, this same technique showed the presence of both cytokines in all tissue samples from every disc hernia patient. However, certain 
differences were found between IL-1 $\beta$ and IL- 6 and among tissues. Quantifications of IL-1 $\beta$ and IL-6 showed no significant differences between painful and nonpainful groups of patients in muscle, AF and NP $(p>0.05)$ (Figure 2). Nevertheless, the protein expression was evidently enhanced in $A F$ for IL-6 and in NP for both cytokines (Figure 2C, 2E and 2F). For IL-6, maximal band intensities of almost two-fold over the reference protein were found in $A F$ and NP (Figure 2E and 2F).

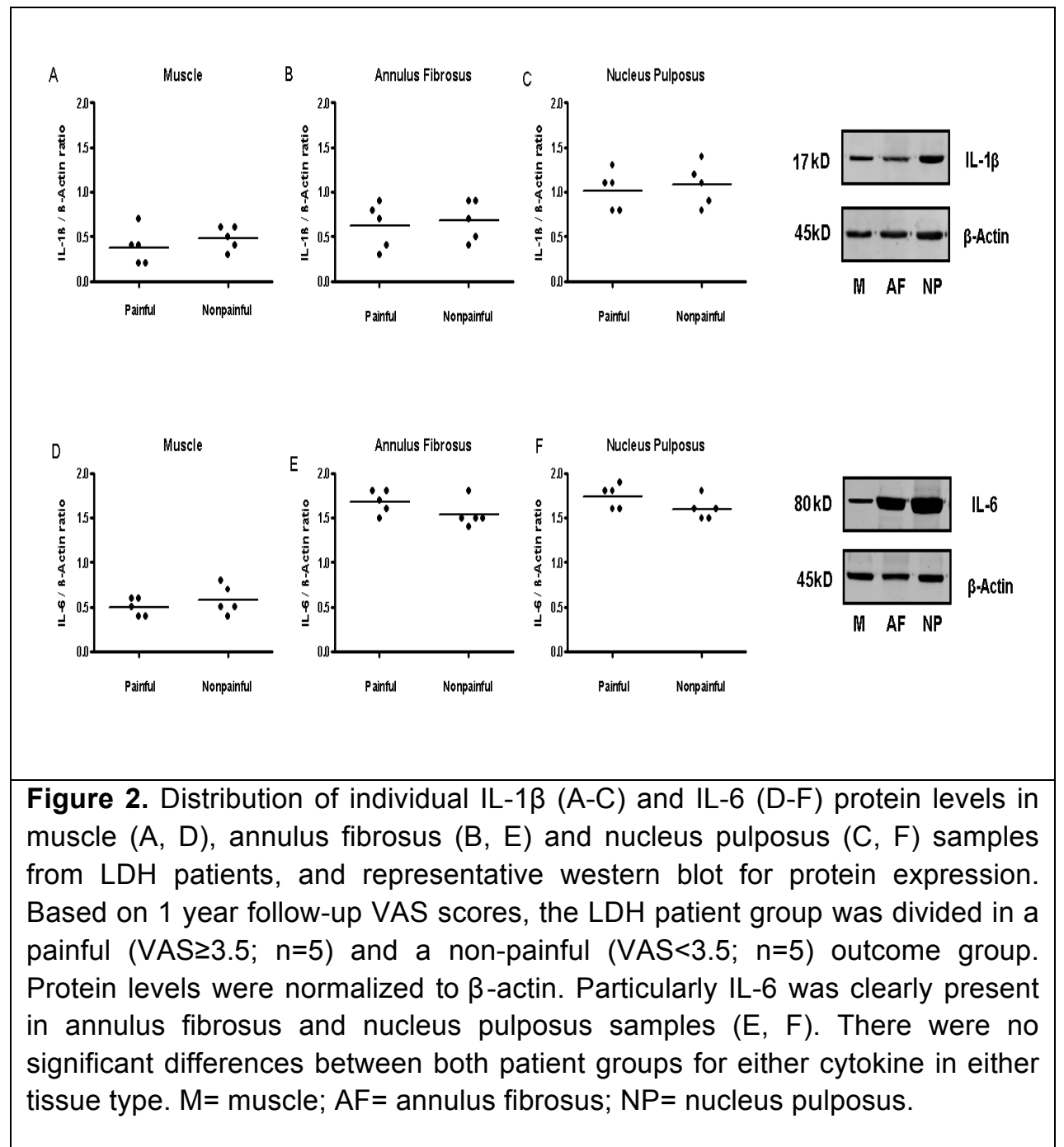




\section{4. Correlation between proinflammatory cytokine levels and pain intensity}

No correlations were performed for paravertebral muscle samples since no significant differences were found in protein and mRNA levels. Correlation between VAS scores at 1 day before surgery, or 6 weeks and 1 year after surgery, and the proinflammatory cytokines mRNA relative expression levels showed no significant correlations among these parameters in painful and nonpainful patients together (Figure 3). As well, IL-1 $\beta$ and IL-6 protein levels showed no correlation with the intensity of pain at 1 day before surgery, or 6 weeks and 1 year postoperative assessment (Figure 4).

A

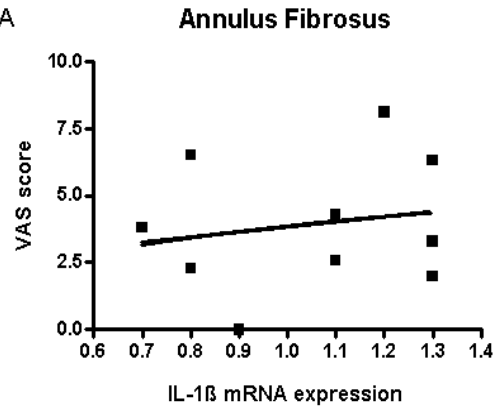

C

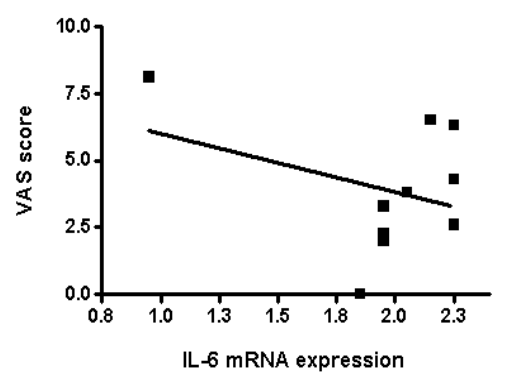

B

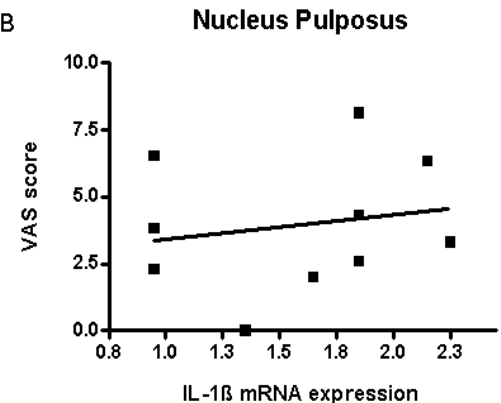

D

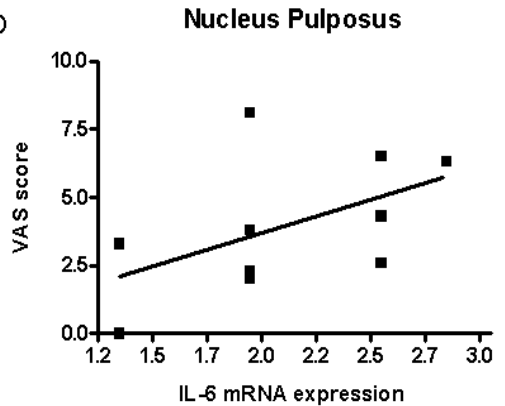

Figure 3. Within the LDH patient group, VAS scores at 1 year follow-up did not correlate with IL-1 $\beta$ mRNA levels in annulus fibrosus $(A, r=0.03), I L-1 \beta$ mRNA levels in nucleus pulposus ( $B, r=0.05), I L-6$ mRNA levels in annulus fibrosus ( $C$, $r=-0.11$ ) or with IL-6 mRNA levels in nucleus pulposus ( $D, r=0.23)$. 


\section{Chapter 5}

\section{Discussion}

This study primarily demonstrates the presence of IL-1 $\beta$ and IL-6 in paravertebral muscle, AF and NP biopsies in LDH patients, but not in control subjects. By detecting proinflammatory cytokines in intervertebral disc biopsies from LDH patients, this study supports the idea that LDH is a condition that is accompanied by a chronic, local inflammation. Yet, the lack of a correlation between IL- $1 \beta$ or IL- 6 levels and the severity of sciatic pain suggest that these cytokines do not play a role in maintaining a pain generating network. In addition, LDH patients in the painful outcome group reported higher VAS scores after 1 year in comparison to the 6 weeks follow-up. This observation could suggest that the acute clinical improvement could be interpreted as a relief of the mechanical compression created by the $\mathrm{LDH}$, and that the persistent progressive pain could be the consequence of a non-resolved neuroinflammatory process. Nevertheless, according to our findings the inflammatory process related to IL-1 $\beta$ and IL- 6 is apparently unspecific and homogenous between all LDH patients, since no significant differences were found between LDH patients with painful and nonpainful outcome.

In recent clinical studies, elevated levels of proinflammatory cytokines (IFN- $\gamma$, IL-1 $\beta$, IL-2, IL-6 and TNF- $\alpha$ ) correlated with elevated pain scores in various types of neurological and rheumatologic disorders [11-20]. In addition, we found that intervertebral TNF- $\alpha$ levels correlate with preoperative pain in LDH patients [21]. In these studies, analysis of inflammatory markers in human samples from peripheral blood, cerebrospinal fluid, periradicular fat tissue, skin punch biopsies, nerve biopsies and lumbar facet joints have demonstrated that both, pro- and anti-inflammatory cytokines play a role in pain control. However, it is difficult to recognize a shared pathophysiological mechanism because these studies investigated a wide variety of different painful conditions. Another unresolved issue about the role of neuroinflammation in pain disorders is the extent to which local and/or systemic expressed cytokines contribute to the clinical symptoms, or if it is disease-specific. For instance, studies of complex regional pain syndrome, painful neuropathies and intervertebral disc degeneration indicate that a local elevation of proinflammatory cytokines is directly related to the amount of pain $[11,13,22]$. Yet studies in patients with neuropathic, nociceptive and mixed types of chronic pain show that elevated peripheral markers related to pain sensation $[12,14,15,19]$. We are equally in the dark regarding the temporal resolution of neuroinflammatory markers during the transition from an initial precipitating event to chronic pain. There is not enough knowledge to establish clear correlations between the duration of the 
inflammatory process and the severity that this temporal exposition would have on pain

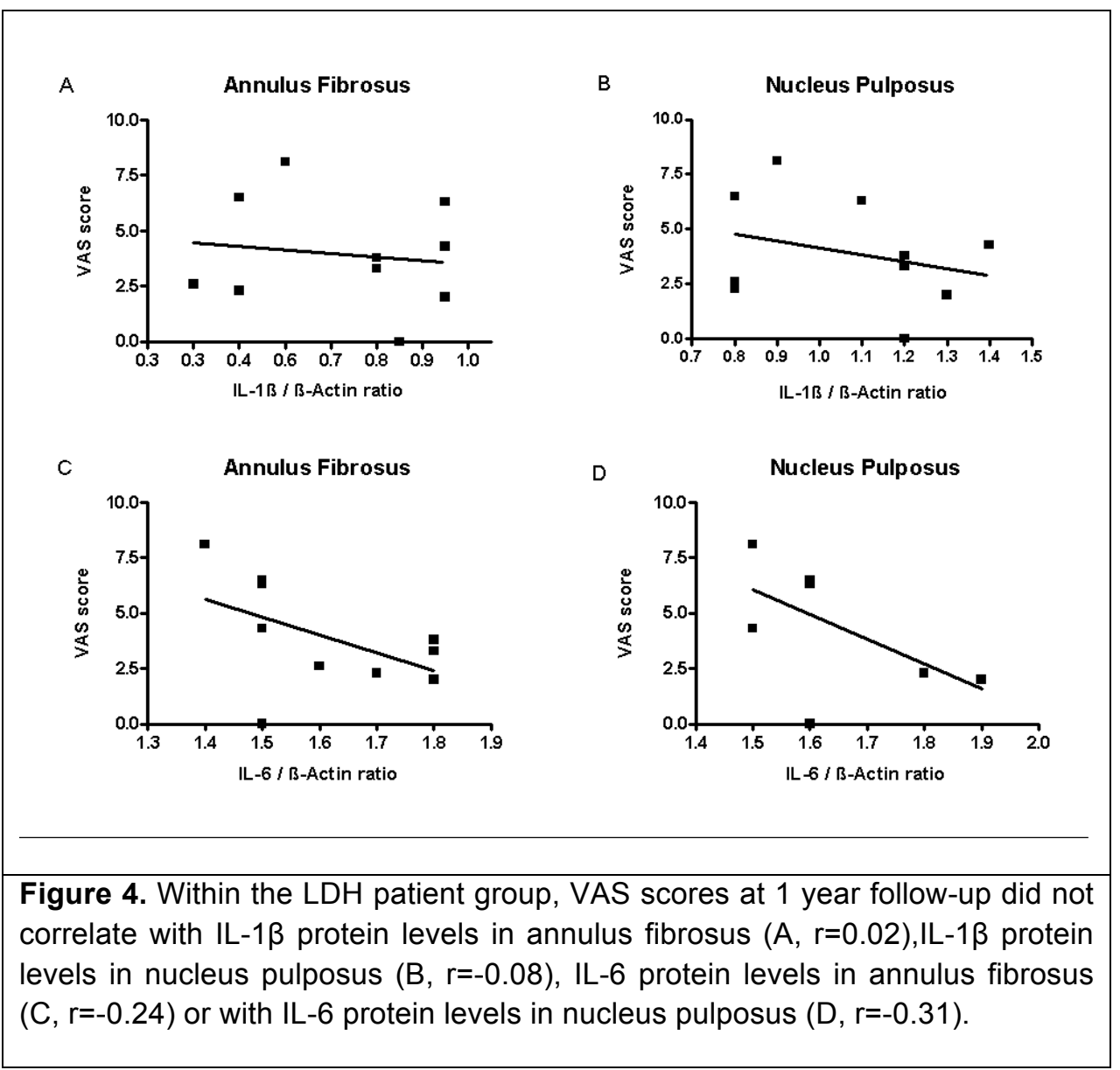

The clinical relevance from previous findings and our data still remains to be determined. The lack of a correlation between locally enhanced IL-1 $1 / / \mathrm{L}-6$ levels and pain sensation is in line with the observation that some antiinflammatory drugs perform poorly as treatment for pain and suggests that it may be more efficient to target the pain-specific inflammatory profile. In this study, protein and mRNA levels of both IL-1 $\beta$ and IL- 6 were clearly elevated. This finding was most pronounced for IL-6, which is in line with the observation that this cytokine is expressed persistently in inflamed tissue [23]. Apparently, 


\section{Chapter 5}

this long-term exposure does not affect the severity of pain, possibly explained by the capacity of IL- 6 to function as an anti-inflammatory cytokine at the same time [24, 25]. Succinctly, IL-6 dual role in both action and time expression could complicate the analysis and therefore the importance of this cytokine in association with pain symptoms. On the other hand, the role of IL-1 $\beta$ is far more complicated since its contribution to pain has been described as a direct mechanical and thermal hyperalgesic agent on nociceptors, and with the induction of other molecules that are involved in pain sensation, including IL-6 [24].

\section{Conclusion}

Intervertebral discs biopsies from LDH patients express proinflammatory cytokines IL-1 $\beta$ and IL-6. The amount of cytokine is however not correlated with the amount of leg pain that is experienced preoperatively or after decompressing surgery. Furthermore, our findings suggest that the acute pain relief obtained by patients could be related to the mechanical decompression, and that the chronic increasing pain could be a consequence of a non-resolved neuroinflammatory process.

\section{References}

1. Valat JP, et al. Sciatica. Best Pract Res Clin Rheumatol 2010;24:241-52.

2. Koes BW, et al. Diagnosis and treatment of sciatica. BMJ 2007;334:1313-7.

3. Vroomen PC, et al. Conservative treatment of sciatica: a systematic review. J Spinal Disord 2000;13:463-9.

4. Legrand E, et al. Sciatica from disk herniation: Medical treatment or surgery? Joint Bone Spine 2007;74:530-5.

5. Üçeyler $\mathrm{N}$, et al. Early cytokine gene expression in mouse CNS after peripheral nerve lesion. Neurosci Lett 2008;436:259-64.

6. Ozaktay AC, et al. Effects of interleukin-1 beta, interleukin-6, and tumor necrosis factor on sensitivity of dorsal root ganglion and peripheral receptive fields in rats. Eur Spine J 2006;15:1529-37.

7. Zhou Z, et al. A novel cell-cell signaling by microglial transmembrane TNFa with implications for neuropathic pain. Pain 2010;151:296-306.

8. DeLeo JA, et al. Cytokine and growth factor immunohistochemical spinal profiles in two animal models of mononeuropathy. Brain Res 1997;759:50-7. 
9. Schäfers $M$, et al. Combined epineurial therapy with neutralizing antibodies to tumor necrosis factor-alpha and interleukin-1 receptor has an additive effect in reducing neuropathic pain in mice. Neurosci Lett 2001;310:113-6.

10. Andrade $\mathrm{P}$, et al. Role of TNF-alpha during central sensitization in preclinical studies. Neurol Sci 2011;32:757-71.

11. Üçeyler N, et al. Differential expression of cytokines in painful and painless neuropathies. Neurology 2007;69:42-9.

12. Koch $A$, et al. Nitric oxide and pro-inflammatory cytokines correlate with pain intensity in chronic pain patients. Inflamm Res 2007;56:32-7.

13. Üçeyler N, et al. Elevated proinflammatory cytokine expression in affected skin in small fiber neuropathy. Neurology 2010;74:1806-13.

14. Wang $\mathrm{H}$, et al. Influence of depression symptoms on serum tumor necrosis factor- $\alpha$ of patients with chronic low back pain. Arthritis Res Ther 2010;12:R186.

15. Ludwig J, et al. Cytokine expression in serum and cerebrospinal fluid in non-inflammatory polyneuropathies. J Neurol Neurosurg Psychiatry 2008;79:1268-73.

16. Empl M, et al. TNF-alpha expression in painful and nonpainful neuropathies. Neurology 2001;56:1371-7.

17. Igarashi A, et al. Correlation between inflammatory cytokines released from the lumbar facet joint tissue and symptoms in degenerative lumbar spinal disorders. J Orthop Sci 2007;12:154-60.

18. Genevay $S$, et al. Elevated levels of tumor necrosis factor-alpha in periradicular fat tissue in patients with radiculopathy from herniated disc. Spine 2008;33:2041-6.

19. Wang $\mathrm{H}$, et al. The role of TNF-alpha in patients with chronic low back paina prospective comparative longitudinal study. Clin J Pain 2008;24:273-8.

20. Ohtori $S$, et al. Proinflammatory cytokines in the cerebrospinal fluid of patients with lumbar radiculopathy. Eur Spine J 2011;20:942-6.

21. Andrade $P$, et al. TNF-alpha levels correlate with postoperative pain severity in lumbar disc hernia patients: Opposite clinical effects between TNF receptor 1 and 2. Pain 2011;152:2645-52.

22. Krämer HH, et al. TNF-alpha in CRPS and 'normal' trauma - Significant differences between tissue and serum. Pain 2010;152:285-90.

23. Studer R, et al. Human Nucleus Pulposus Cells React to IL-6: independent actions and amplification of response to IL-1 and TNF- $\alpha$. Spine 2011;36:593-9.

24. Watkins LR, et al. Immune activation: the role of pro-inflammatory cytokines in inflammation, illness responses and pathological pain states. Pain 1995;63:289-302. 
Chapter 5

25. Xing Z, et al. IL-6 is an antiinflammatory cytokine required for controlling local or systemic acute inflammatory responses. J Clin Invest 1998;101:311-20. 


\section{Chapter}

\section{Elevated levels of TNF- $\alpha$ and TNFR1 in recurrent herniated lumbar discs correlate with chronicity of postoperative sciatic pain}

Pablo Andrade, Govert Hoogland, Onno P. Teernstra, Jasper van Aalst, Emiel van Maren, Marc A. Daemen, Harry W.M. Steinbusch, Veerle Visser-Vandewalle 


\section{Chapter 6}

\section{Abstract}

Sciatica is a condition characterized by radicular pain along the trajectory of the sciatic nerve, which can be secondary to a lumbar disc herniation (LDH). In more than $10 \%$ of patients who underwent discectomy, persistent sciatic pain occurs. The underlying mechanisms of sciatica after discectomy remain unclear. There is evidence demonstrating that inflammation plays a role in the pathophysiology of sciatica. This study evaluated the correlation between the level of inflammation in the intervertebral disc (IVD) with pre- and postoperative pain scores after discectomy in LDH patients operated for the first time and recurrent cases. We analyzed mRNA and protein levels of TNF- $\alpha$, TNFR1 and TNFR2 in IVD biopsies, and correlated these levels with visual analogue scale (VAS) scores from 1 day before surgery and 6 weeks and 6 months postoperatively. This analysis showed that TNF- $\alpha$ and TNFR1 mRNA levels were significantly greater in recurrent LDH patients than in first-time operated patients; 2 -fold increase for TNF- $\alpha$ and $50 \%$ increase for TNFR1. Similarly, protein levels in IVD samples positively correlated with postoperative VAS scores, whereas TNFR2 protein levels negatively correlated with postoperative VAS scores. These findings indicate that recurrent LDH patients do not only present higher postoperative VAS scores, compared with patients who underwent LHD for the first time, but also that these scores are correlated with increased levels of inflammation and may be responsible for the chronicity of pain. 
TNF-alpha levels in recurrent LDH patients correlate with chronic pain

\section{Introduction}

Sciatica is a relatively frequent condition characterized by radicular pain along the trajectory of the sciatic nerve; normally, it is only one lumbar nerve root that is affected. The symptoms responsible for this condition arise as a consequence of a single or multiple disc herniations that compress the nerve roots. Normally, the painful sensation is felt in the low back, leg and the foot, it can also be accompanied by dysesthesias, paresthesias and weakness. This condition represents one of the leading causes of chronic pain in adults with a lifetime incidence of up to $40 \%$, representing a major socio-economic burden (Frymoyer, 1988). Epidemiological studies have demonstrated that the majority of sciatic pain episodes resolve spontaneously or with the help of conventional analgesics and physiotherapy (Stafford et al., 2007; Peul et al., 2007). However, in cases where the symptoms persist for more than 6-8 weeks different approaches are considered for treatment (eg, surgery) (Valat et al., 2010). In cases where the pain is attributed to a lumbar disc herniation (LDH), decompressive discectomy is usually considered one of the best options to relieve the constriction and alleviate the pain. However, the sole mechanical compression of a prolapsed intervertebral disc cannot explain in all cases the pathogenesis of sciatic pain. More than one in ten patients report chronic and intractable pain after undergoing single or multiple spine surgery to remove the affected disc (Carragee et al., 1999). These unsuccessful cases suggest that other mechanisms may play an important part in the pathogenesis of chronic sciatic pain.

Inflammation has largely been considered as an important factor in the modulation of pain. This concept is based on a large body of preclinical and clinical evidence demonstrating the nociceptive action of proinflammatory cytokines on nervous tissue (De Leo et al., 1997; Schäfers et al., 2003a and 2003b Koch et al., 2007; George et al., 1999; Üçeyler et al., 2007). Among these agents, TNF- $\alpha$ has been shown to be a key player in the development and maintenance of pain (DeLeo et al., 2000; Youn et al., 2008; Sacerdote et al., 2008). In addition, in recent years the participation of both TNF- $\alpha$ receptors (TNFR), TNFR1 and TNFR2, has been associated in pathways that can affect pain sensation (Sakuma et al., 2007; Constantin et al., 2008). After TNF- $\alpha$ stimulation, these TNFR have been shown to activate independent opposite mechanisms that ultimately result either in apoptosis for TNFR1 or in cell survival for TNFR2 (Aggarwal, 2003; Zhang et al., 2011). However, the participation of TNF- $\alpha$ and TNFR as modulators in the generation of pain is not 
fully understood. In addition, how these different intracellular pathways can influence the severity of pain in patients still remains unclear.

In a previous study from our group, we provided evidence that the presence of TNF- $\alpha$ in disc biopsies from LDH operated patients could predict the outcome after surgery (Andrade et al., 2011b). Furthermore, we reported that increased TNFR1 and decreased TNFR2 levels in these disc biopsies were associated with increased postoperative pain scores. The aims of the present study were; firstly, to assess if the expression of TNF- $\alpha$ and TNFR were correlated with the severity of pre- and postoperative leg pain in LDH patients that underwent single or multiple decompressive discectomies. Finally, to investigate if TNF- $\alpha$ and TNFR expression are associated with surgery resistant cases in regard to chronic pain sensation.

\section{Patients and Methods}

The ethics committee of Maastricht University Medical Center approved all human procedures included in this study, and informed written consent was obtained from each individual.

\section{1. Patients and biopsy collection}

Patients were diagnosed with LDH after neurological examination and confirmation of the affected disc level seen on magnetic resonance imaging (MRI). Patients had leg pain for at least 3 months before undergoing elective spinal surgery. In total, 20 patients with a single-level disc herniation were included in this study. Two different groups were composed; group 1 consisted of twelve patients who underwent surgery for $L D H$ for the first time (fLDH), and group 2 consisted of eight patients who underwent surgery for LDH for a second time at the same disc level, recurrent cases (rLDH). The latter were considered as recurrent herniation since an unsatisfactory pain outcome was present over 1 year after the previous operation. Patients who had previously undergone spinal surgery for another indication were excluded. Patients had anti-inflammatory medications suspended for at least on week before the surgery. From each patient intervertebral disc (IVD) biopsies were collected during surgery, and were immediately frozen in liquid nitrogen and then stored at $-80^{\circ} \mathrm{C}$. 
TNF-alpha levels in recurrent LDH patients correlate with chronic pain

\section{2. Pain score}

Patients were evaluated using Visual Analogue Scale (VAS) scores $(0=$ no pain; $10=$ worst pain ever experienced) in order to measure their level of leg pain at different pre- and postoperative time points. The scores were recorded one day before the operation, and six weeks and six months after the surgery. In all patients, the leg pain was considered as secondary to a single-disc herniation involvement.

\section{3. $q P C R$}

All tissue biopsies were cut in two equal parts, one for Western blot analysis (see below) and one for qPCR. A detailed description of the qPCR technique has been reported previously (Andrade et al., 2011b). In brief, total RNA was extracted using the reagent TRIzol (Invitrogen, CA) according to the manufacturer's protocol. $1 \mu \mathrm{g}$ total RNA was reversely transcribed to cDNA using a First Strand cDNA Synthesis Kit (Fermentas International Inc., Ontario, Canada). TNF- $\alpha$, TNFR1 and TNFR2 mRNA expression were measured using a LightCycler 480 Real-Time PCR System adding SYBR Green LightCycler 480 SYBR Green I Master (Roche Diagnostics) for 50 cycles in a fixed sequence of $94{ }^{\circ} \mathrm{C}$ for $30 \mathrm{~s}, 63^{\circ} \mathrm{C}$ for $15 \mathrm{~s}$, and $72^{\circ} \mathrm{C}$ for $15 \mathrm{~s}$. The following primer sets were used: TNF-a-forward (5'-TTCCTGATCGTGGCAGGC-3') and reverse (5'GCTGATTAGAGAGAGGTCCCTG-3'), ACCAAGTGCCACAAAGGAAC-3') CTGCAATTGAAGCACTGGAA-3'), TTCGCTCTTCCAGTTGGACT-3') TNFR1-forward and TNFR2-forward CACCAGGGGAAGAATCTGAG-3'). Amplicons for the reference gene glyceraldehyde 3-phosphate dehydrogenase (GAPDH) were generated using the following primer sets: forward (5'-ACAGTCAGCCGCATCTTC-3') and reverse (5'-CCAATACGACCAAATCCGTTG-3'). Quantification was performed with the delta CT method using the mentioned reference gene.

\section{4. Western blot}

A detailed description of the western blot technique has been reported previously (Andrade et al., 2011b). In brief, tissue was homogenized in ice-cold lysis buffer. The same amount of protein $(20 \mu \mathrm{g})$ was loaded in each lane, then 
separated by SDS-10\% polyacrylamide gel electrophoresis for 90 min at $100 \mathrm{~V}$ and then transferred to a pure nitrocellulose membrane (Bio-Rad Laboratories, $\mathrm{CA}$ ) for $1 \mathrm{~h}$ at $100 \mathrm{~V}$. The membranes were rinsed with PBS and blocked in Odyssey blocking buffer (LI-COR Biosciences, NE) 1:2 diluted in PBS for $1 \mathrm{~h}$, and were incubated overnight at $4^{\circ} \mathrm{C}$ with monoclonal mouse-anti human TNF- $\alpha$ (1:200; 52B83, Santa Cruz Biotechnology, CA), polyclonal rabbit-anti human TNF-R1 (1:500; H-271 Santa Cruz Biotechnology), and polyclonal rabbit-anti human TNF-R2 (1:500; H-202, Santa Cruz Biotechnology). Monoclonal mouseanti human $\beta$-Actin (1:500; ACTBD11B7, Santa Cruz Biotechnology) was used as a loading protein control. Subsequently, membranes were rinsed with PBS and incubated for $1 \mathrm{~h}$ with 1:10.000 Alexa Fluor 800 goat anti-rabbit IgG (Molecular Probes, OR) and 1:10.000 Alexa Fluor 680 donkey anti-mouse IgG (Molecular Probes, OR). Finally, membranes were rinsed with PBS, dried and visualized by enhanced chemiluminescence detection with an Odyssey Infrared Imaging System (LI-COR Biosciences). Immunoreactive bands were quantified by calculating the optical density ratio of the band of interest over that of $\beta$ Actin, using ImageJ 1.42q software (National Institute of Health, MD).

\section{5. Statistical analysis}

All data in this study were expressed as mean \pm SEM (standard error of the mean) and analyzed using GraphPad Prism 5 software with p-values $<0.05$ considered as significant. Differences in TNFR mRNA and protein expression between groups were evaluated by a Student's t-test. In order to estimate the correlation between TNFR expression and pain, data were analyzed using a linear regression analysis, where western blot values were correlated with VAS scores.

\section{Results}

\section{1. Patient characteristics and pain assessment}

Clinical data and demographic characteristics from all LDH operated patients are presented in Table 1; last section of the thesis. Twenty subjects (mean age $49.8 \pm 3.1$ years, 7 females) were included in this analysis. No significant differences in age were found between the groups of first time $(n=12)$ and recurrent $(n=8) \mathrm{LDH}$ patients. Clinical findings and imaging studies showed a 
TNF-alpha levels in recurrent LDH patients correlate with chronic pain

distribution of the LDH on the right side in $45 \%$ of the cases and in the left side in $55 \%$ of the cases. The most affected disc level was L5-S1 in $50 \%$ of the cases, followed by L4-L5 in 40\% of the cases, and finally L3-L4 in 10\% of the cases. Pain assessment using the VAS showed a baseline score 1 day before discectomy that ranged from 5.5 to 9.4 points (median 7.8 points). After 6 weeks follow-up, VAS scores ranged from 1.1 to 4.6 points (median 3.45 points), showing an improvement compared to baseline measurements that ranged from 3.0 to 7.8 points (mean 4.9 points). After 6 months follow-up, VAS scores ranged from 0.0 to 6.8 points (median 3.7 points), showing an improvement compared to baseline measurements that ranged from 1.0 to 9.3 points (mean 4.3 points).

Expressed in percentage, the recovery after 6 weeks follow-up ranged from $40 \%$ to $85 \%$ (mean $61 \%$ ), and the recovery after 6 months follow-up ranged from $13 \%$ to $100 \%$ (mean $54 \%)$. If data from fLDH $(n=12)$ and rLDH $(n=8)$ patients were analyzed separately, the recovery in VAS scores after 6 weeks follow-up ranged from 3.0 to 7.8 points (mean 5.0 points) for the fLDH group, and from 3.1 and 6.4 points (mean 4.8 points) for the rLDH group. After 6 months follow-up, VAS scores ranged from 2.2 and 7.7 points (mean 4.5 points) for the fLDH group, and from 1.4 and 8.3 points (mean 4.1 points) for the rLDH group.

\section{2. TNF- $\alpha$ and TNFR mRNA expression}

After normalizing samples to GAPDH expression, TNF- $\alpha$ mRNA in samples from rLDH patients was 2-fold increased compared to those of fLDH patients (Fig. 1A). Similarly, TNFR1 mRNA levels in samples from rLDH patients showed a significant $50 \%$ increase on the relative expression compared to those of fLDH patients (Fig. 1B). Whereas TNFR2 mRNA levels in biopsies were comparable between the two study groups and no significant differences were found (Fig. 1C). To investigate if the leg pain was accompanied by an altered mRNA expression, we compared the individual relative expression of $f+r L D H$ patients (both groups together) with a successful outcome (VAS score < 3.5 points; $n=10$ for 6 weeks, and $n=10$ for 6 months) with that of $f+r L D H$ patients with an unsuccessful outcome (VAS score $\geq 3.5$ points; $n=10$ for 6 weeks, and $\mathrm{n}=10$ for 6 months). When patients were grouped based on their VAS score at 6-week (data not shown) and 6-month follow-up, TNF- $\alpha$ and 
Chapter 6

TNFR1 mRNA expression was increased and TNFR2 was decreased in samples from patients with VAS scores $\geq 3.5$ points (Fig. 2).

A
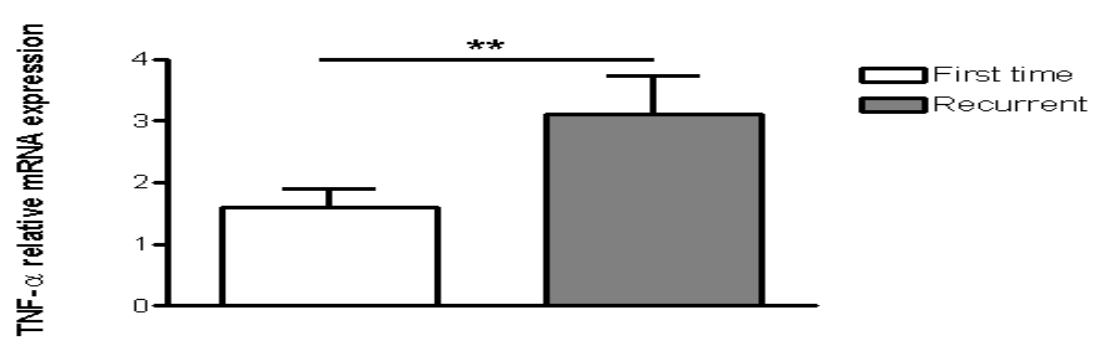

$\mathbf{B}$
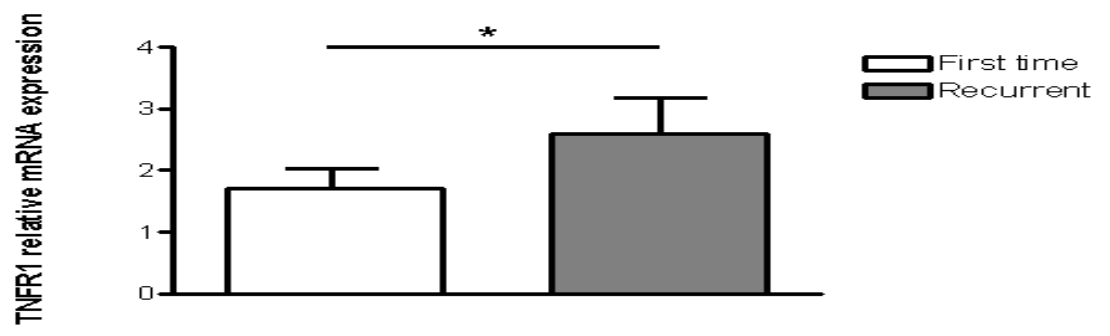

$c$
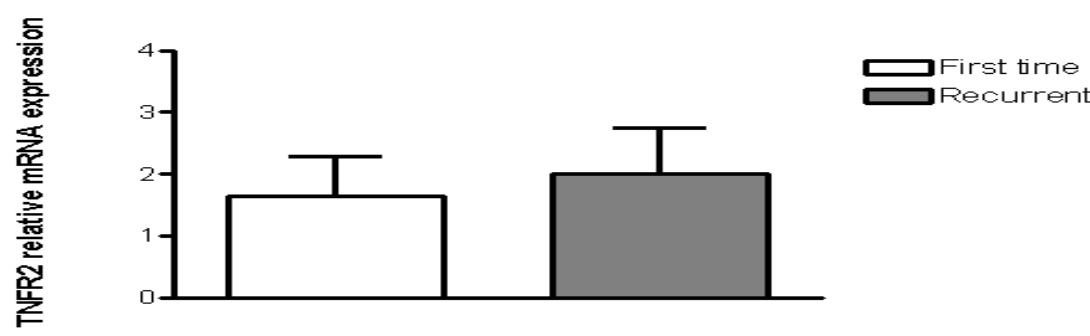
Recurrent

Figure 1. Relative TNF- $\alpha$ and TNFR mRNA expression in first time (white bars) and recurrent (grey bars) LDH operated patients. Relative TNF- $\alpha$ and TNFR1 mRNA expression in IVD samples were significantly increased in recurrent LDH patients, compared to those of first time-operated patients $(A, B)$. The mRNA expression is graphed as time-fold increase after normalizing samples to GAPDH expression. Results are expressed as mean ( \pm SEM) from 10 patients per group. ${ }^{*} p<0.05,{ }^{* *} p<0.01$. 
TNF-alpha levels in recurrent LDH patients correlate with chronic pain

\section{3. TNF- $\alpha$ and TNFR protein expression}

To corroborate the findings obtained through qPCR, Western Blot was performed on the same tissue samples obtained during surgery. Transmembrane TNF- $\alpha$ (mTNF- $\alpha$ ), TNFR1 and TNFR2 were clearly detectable in all samples from both study groups included in this study. Similar to mRNA data, differential TNF- $\alpha$ and TNFR expression was observed between the two groups (Fig. 3). Optical density quantifications showed that samples from rLDH patients expressed significant higher TNF- $\alpha$ and TNFR1 levels compared to those of fLDH patients. Whereas TNFR2 quantification showed that the highest expression was present in samples from fLDH. To investigate whether pain sensation was related to inflammation in the IVD, we analyzed the correlation between baseline VAS scores 1 day before surgery and TNF- $\alpha$ and TNFR protein expression in the samples. This analysis learned that TNF- $\alpha$ and TNFR levels did not correlate with the preoperative VAS scores in $f+r L D H$ patients (Fig. 3C, E, G). In order to determine if a local inflammation in the IVD would have a predictive value on the discectomy recovery in terms of pain sensation, we analyzed the correlation between TNF- $\alpha$ and TNFR protein expression in the samples and postsurgical VAS scores at 6-week and 6-month follow-up. This analysis learned that TNF- $\alpha$ and TNFR levels moderately correlated with VAS scores at 6 months in $\mathrm{f}+\mathrm{rLDH}$ patients (Fig. 3D, F, H), but not at 6 weeks (not shown in the figure). Elevated levels of TNF- $\alpha$ and TNFR1 correlated with high VAS scores at 6-month follow-up ( $r=0.52$ and $r=0.50$, respectively; $p<0.05$ for both). Moreover, elevated levels of TNFR2 were correlated with low VAS scores at 6-month follow-up $(r=-0.54 ; p<0.01)$.

\section{4. Differential TNFR expression levels}

To investigate if leg pain was accompanied by a distinctive or similar modified TNFR expression, we analyzed the TNFR1 / TNFR2 mRNA and protein ratio signature of $f+r L D H$ patients when they were grouped according to their VAS score. At 6 months after surgery the samples showed an approximately 4-fold higher mRNA expression of TNFR1 over TNFR2 (Fig. 4A), and an approximate 2 -fold increase in the protein expression of the former over the latter (Fig. 4B). Similarly, if data from fLDH and rLDH were analyzed separately, TNFR1 / TNFR2 ratios in samples from rLDH patients showed a significant increase of approximate $60 \%$ in the protein expression in comparison to samples from fLDH patients (Fig. 4D). However, this same analysis showed no statistical 
differences between groups in the case of TNFR1 / TNFR2 ratios for mRNA expression (Fig. 4D).

$\mathbf{A}$
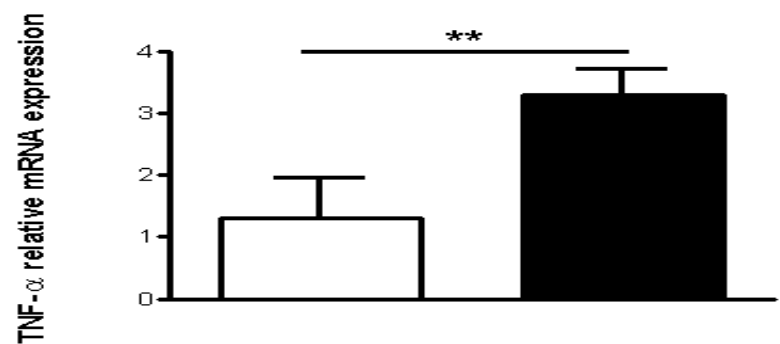

己 $23.5 \vee$ AS score $\geq 3.5$ VAS score

$\mathbf{B}$
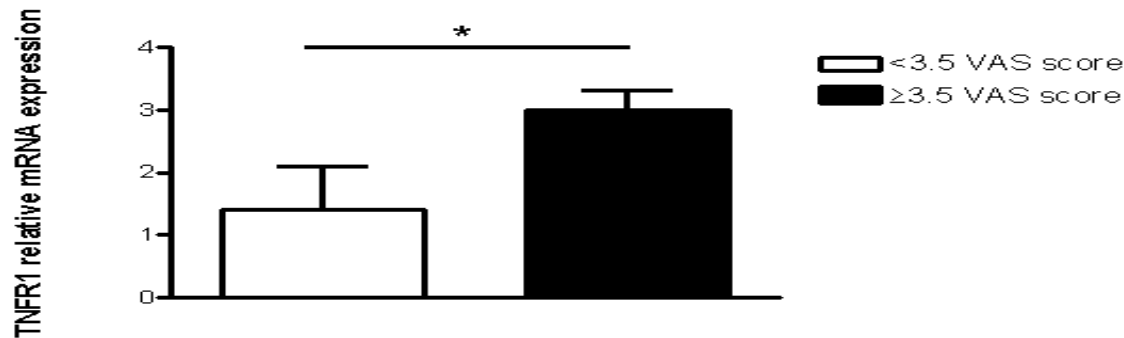
$\geq 3.5$ VAS score

C

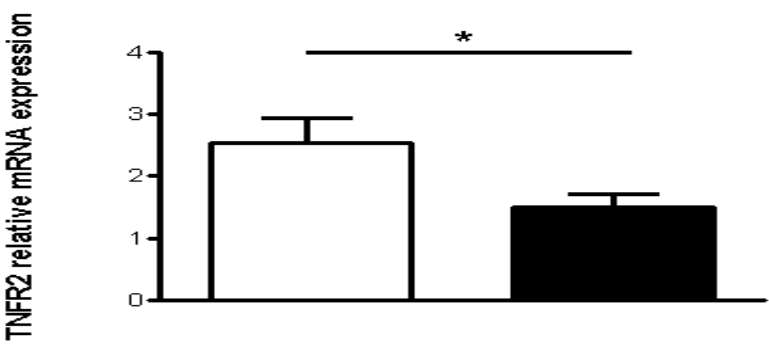

ए3.5 $<$ AS score $\geq 3.5 \vee A S$ score

Figure 2. TNF- $\alpha$ and TNFR mRNA expression in LDH patients with VAS scores $<3.5$ points (white bars) and with VAS scores $\geq 3.5$ points (black bars) at 6 months. Relative TNF- $\alpha$ and TNFR1 mRNA expression in IVD samples were significantly increased in patients with VAS scores $\geq 3.5$ points, compared to those of patients with VAS scores $<3.5$ points $(A, B)$. In contrast, TNFR2 mRNA expression was significantly increased in the samples of patients with VAS scores < 3.5 points compared with the samples of patients with VAS scores $\geq$ 3.5 points $(C)$. The mRNA expression is graphed as time-fold increase after 
TNF-alpha levels in recurrent LDH patients correlate with chronic pain

normalizing samples to GAPDH expression. Results are expressed as mean $( \pm$ SEM) from 10 patients per group. ${ }^{*} p<0.05,{ }^{* *} p<0.01$.

\section{Discussion}

Consistent with previous reports (Genevay et al., 2008; Burke et al., 2002; Kang et al., 1996), the present study indicates that there is an association between the levels of inflammation and the severity of leg pain. Our data show that high levels of the proinflammatory cytokine TNF- $\alpha$ and its receptor TNFR1 in IVD biopsy samples from $\mathrm{f}+\mathrm{rLDH}$ patients are correlated with increased postoperative pain at the long-term outcome. These findings are line with a previous study from our group in which we observed this correlation until one year after surgery in biopsies from annulus fibrosus and nucleus pulposus from LDH patients who underwent spinal surgery for the first time (Andrade et al., 2011b). Furthermore, the present data also corroborates our previous findings in respect to the expression of TNFR2 and its association with low intensity or absence of pain sensation after surgery. Interestingly, in both reports, the preoperative pain assessment scores from LDH patients were not correlated with these cytokine levels. A possible explanation for this observation is that all patients reported considerable high intensity of pain one day before surgery. The resulting lack of variation in preoperative VAS scores impeded easy statistical analysis in contrast to the much more dispersed postsurgical VAS scores.

Previous preclinical studies demonstrated that central sensitization could be enhanced and maintained by the action of TNF- $\alpha$ and its receptors (Zhang et al., 2011; Xu et al., 2006; Bianchi et al., 2007; Covey et al., 2002). In the present study, we observed a lower intensity of pain at 6-week and 6-month follow-up in $f+r L D H$ patients whose IVD samples expressed low TNF- $\alpha$ and TNFR1. Furthermore, our data show that $\mathrm{rLDH}$ patients have significantly increased TNF- $\alpha$ and TNFR1 levels in IVD samples compared to those from $\mathrm{fLDH}$ patients. This is in line with the observations that rLDH patients were the group that reported higher VAS scores at every pain assessment period. One possible explanation for this increase is that the cellular damage to the nerve roots produced by the inflammatory process modulated by TNF- $\alpha$ could determine the absence of pain recovery after creating a permanent injury. It is known that rLDH patients have the capacity to generate ubiquitous scar tissue at the site of a previous discectomy procedure. Although this fibrotic tissue does 


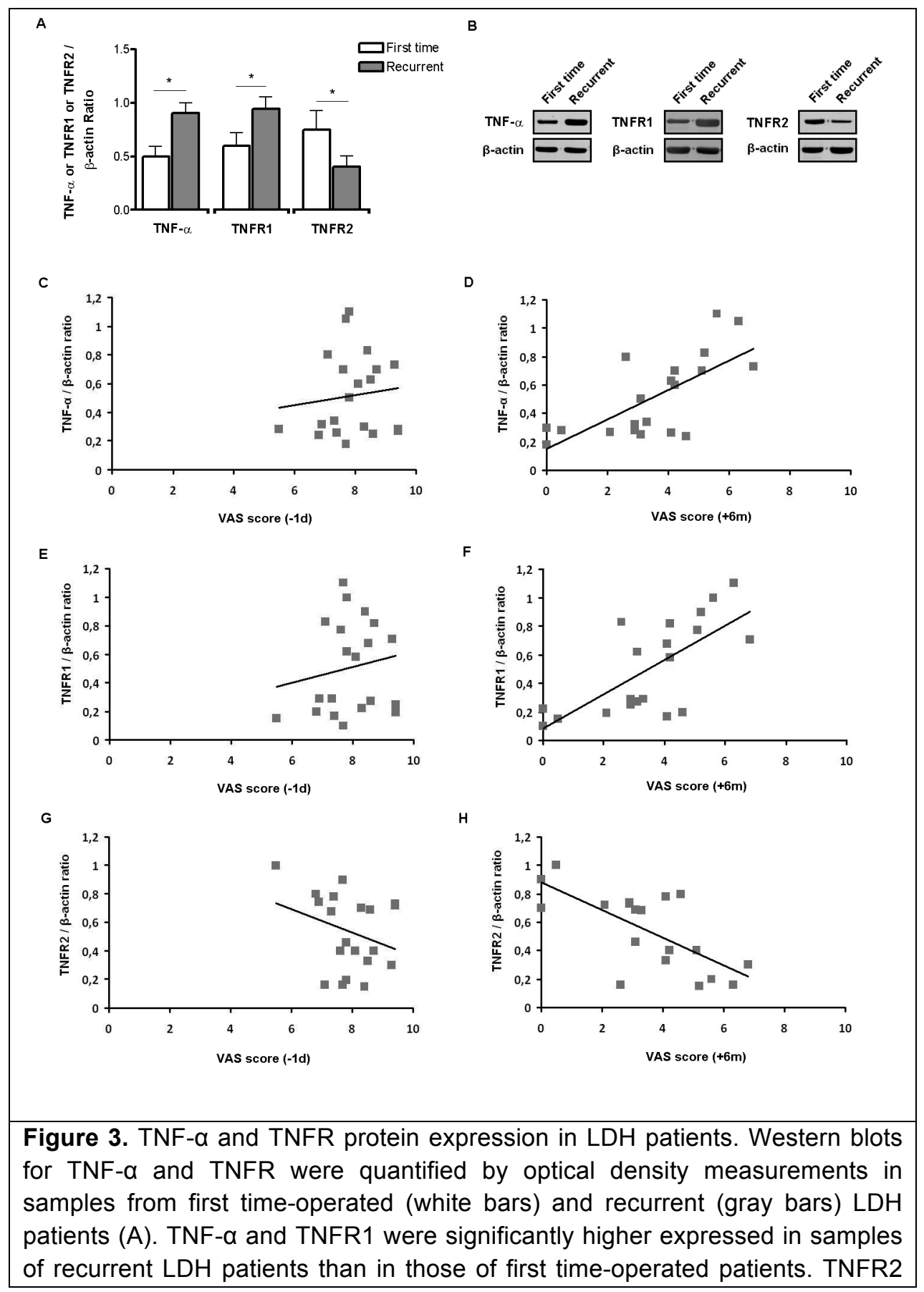


immunoreactive bands were less expressed, but enough to be significantly higher in samples from first time-operated patients compared with the samples of recurrent LDH patients. Results are expressed as mean ( \pm SEM) from 10 patients per group. ${ }^{*} p<0.05$. Representative Western blots showing TNF- $\alpha$ (26 $\mathrm{kDa})$, TNFR1 (55 kDa), TNFR2 (75 kDa) and $\beta$-actin (45kD) expression in IVD samples from first time-operated and recurrent LDH patients (B). Correlation between VAS scores at 1 day before surgery $(C, E, G)$ and at 6 -month postoperative follow-up $(D, F, H)$, and TNF- $\alpha$ and TNFR protein expression in IVD samples. For TNF- $\alpha$ and TNFR1, a positive correlation was found at 6 months after surgery ( $r=0.52$ and $r=0.50$, respectively) and not at 1 day before the procedure $(r=0.01$ and $r=0.02$, respectively). In contrast, TNFR2 protein expression in IVD samples correlated negatively with pain at the 6-month follow-up ( $r=-0.54)$, whereas at 1 day before the surgery there was no correlation $(r=-0.08)$. $-1 d=1$ day before surgery; $+6 m=6$ months after surgery.

not contain nerve endings and normally it is not large enough to compress the roots, it has been largely associated with postoperative pain (Fritsch et al., 1996; Almeida et al., 2008). It has been shown that different cells that surround the site of a LDH (eg, histiocytes, fibroblast, endothelial cells and chondrocytes) have the capacity to produce diverse proinflammatory cytokines, including TNFa (Takahashi et al., 1996). In addition, inflammatory cells that are not originally from the IVD (eg, macrophages and mast cells) have been found to infiltrate herniated discs (Virri et al., 1994; Habtemariam et al., 1999). Therefore, it is possible that the chronic exposition to an enhanced inflammatory process created by the fibrotic tissue in rLDH patients is responsible for the increased leg pain intensity at the long-term. Thus, the clinical outcome of these patients may be limited after multiple consecutive discectomies. Consequently, the surgical prognosis should be elaborated accordingly, in order to select the best candidates for this procedure and use different therapeutic approaches for resistant cases where a high inflammatory profile has been detected.

TNFR have been shown to induce different signaling mechanisms by following distinct intracellular pathways. Although they can have intracellular crosstalk, TNFR1 has been described as a mediator for cell death, whereas TNFR2 has been implicated in cell survival (Yang et al., 2002; Andrade et al., 2011a). In the present study, the increased expression of TNFR1 in rLDH patients was associated with a poor pain recovery after the surgery. In contrast, patients with lower pain scoring (mainly fLDH patients) showed significantly decreased 


\section{Chapter 6}

TNFR1 levels in IVD biopsies. Moreover, high TNFR2 levels correlated with low postsurgical pain scores, suggesting a protective role in the mediation of inflammatory pain. In addition, the TNFR1/TNFR2 signature ratio corroborated that after an individual analysis in cases where TNFR1 expression was elevated over TNFR2, LDH patients from both groups showed high pain scores after the surgery. Nonetheless it is important to remark that this tendency was higher in the rLDH-operated patient group. This is significant because this predisposition to an imbalanced expression between receptors could be in part responsible for failed back surgery syndrome. These observations are consistent with the fact that TNFR1 stimulation has been largely associated with pain generation, while the selective activation of TNFR2 did not reproduce these effects (Lee et al., 2004; Holmes et al., 2004; Sakuma et al., 2007; Schäfers et al., 2008). However, it is still unclear why certain patients are likely to express one receptor over the other, and it would certainly be interesting to investigate the mechanisms behind these tendencies. Subsequent studies will need to address the question of this shift on the receptor expression. Our findings also suggest that despite the intracellular crosstalk between TNFR, eventually the ultimate tissue expression levels in IVD samples maintain a close correlation with the prototypical effect of each receptor to induce pain or not. This means that, under normal conditions the stimulation of TNFR1 in the nerve roots will culminate in the majority of cases in an increase on the pain scores, and on the contrary, TNFR2 activation will result in lower pain scores.

Taken together, the present study indicate that LDH patients who underwent multiple spinal surgeries do not only tend to report higher pain scores, but also that these scores are correlated with increased levels of inflammation and probably to cell death. These findings provide more evidence that local IVD inflammation (TNF- $\alpha$ and TNFR1) in LDH patients is closely involved in postsurgical leg pain. Additional research is needed to establish the apparent protective role of TNFR2 on chronic pain. However, according to our observations, this receptor can be associated with low pain scores or even the absence of pain in certain cases. Finally, the distinct role of TNFR in relation to predict the outcome after disc surgery might have therapeutic implications in the modulation of pain. It is important to remark that, although this study focused on the key inflammatory agents TNF- $\alpha$ and its two receptors, our findings cannot exclude the involvement of other proinflammatory cytokines on the generation of leg pain in LDH patients. 
TNF-alpha levels in recurrent LDH patients correlate with chronic pain

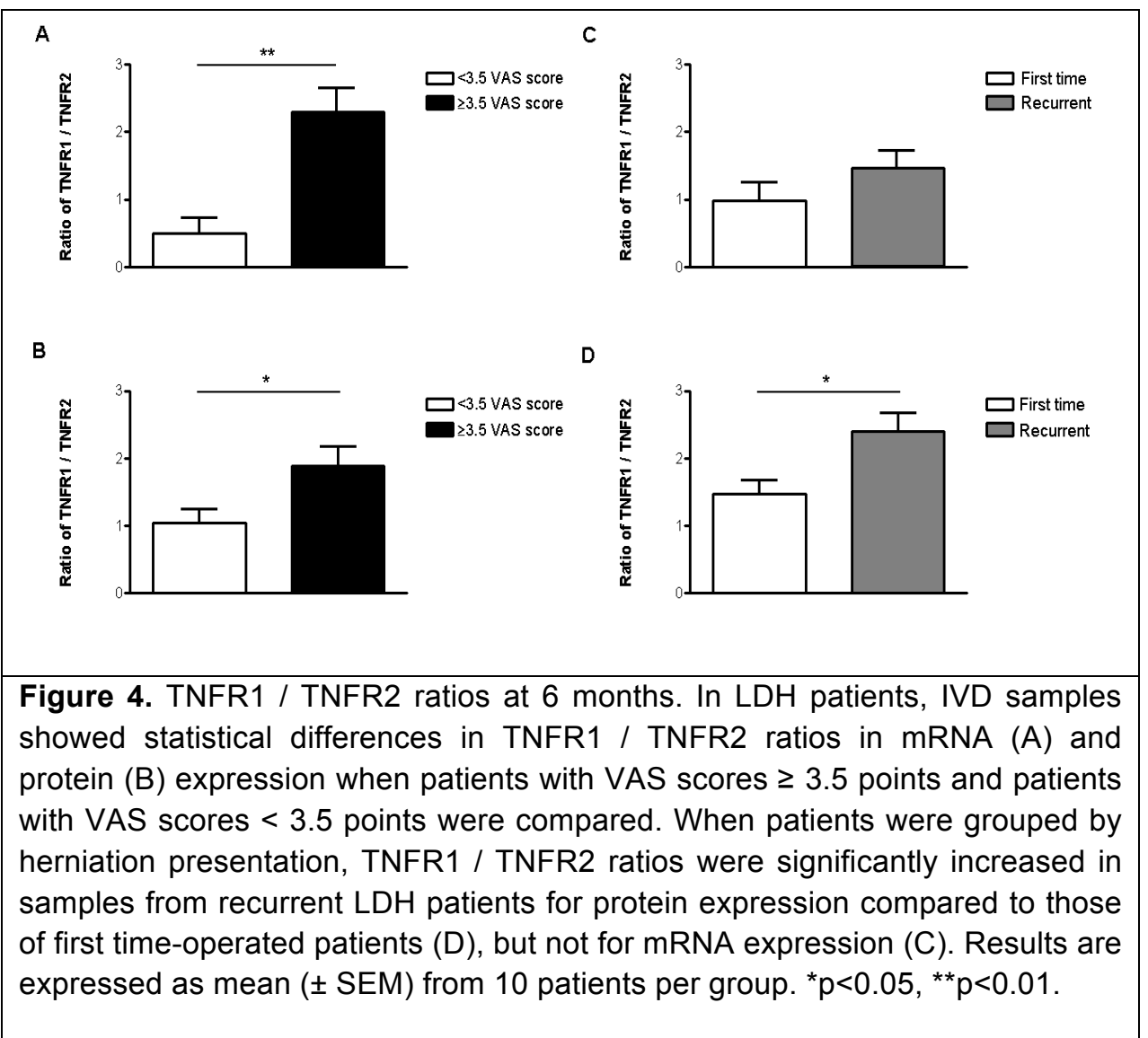

\section{References}

1. Aggarwal BB. Signalling pathways of the TNF superfamily: a doubleedged sword. Nat Rev Immunol 2003;3:745-56.

2. Almeida $\mathrm{DB}$, et al. Outcome following lumbar disc surgery: the role of fibrosis. Acta Neurochir (Wien) 2008;150:1167-76.

3. Andrade $P$, et al. The thalidomide analgesic effect is associated with differential TNF- $\alpha$ receptor expression in the dorsal horn of the spinal cord as studied in a rat model of neuropathic pain. Brain Res 2012;1450:24-32.

4. Andrade $P$, et al. Role of TNF-alpha during central sensitization in preclinical studies. Neurol Sci 2011a;32:757-771. 
5. Andrade $P$, et al. Tumor necrosis factor-alpha levels correlate with postoperative pain severity in lumbar disc hernia patients: Opposite clinical effects between tumor necrosis factor receptor 1 and 2. Pain 2011b;152:2645-2652.

6. Bianchi $M$, et al. Increased tumor necrosis factor-alpha and prostaglandin E2 concentrations in the cerebrospinal fluid of rats with inflammatory hyperalgesia: the effects of analgesic drugs. Anesth Analg 2007;104:949-54.

7. Burke JG, et al. Intervertebral discs which cause low back pain secrete high levels of proinflammatory mediators. J Bone Joint Surg $\mathrm{Br}$ 2002;84:196-201.

8. Carragee EJ, et al. Activity restrictions after posterior lumbar discectomy. A prospective study of outcomes in 152 cases with no postoperative restrictions. Spine 1999;24:2346-51.

9. Constantin $\mathrm{CE}$, et al. Endogenous tumor necrosis factor alpha (TNFalpha) requires TNF receptor type 2 to generate heat hyperalgesia in a mouse cancer model. J Neurosci 2008;28:5072-5081.

10. Covey WC, et al. Expression of neuron-associated tumor necrosis factor alpha in the brain is increased during persistent pain. Reg Anesth Pain Med 2002;27:357-66.

11. DeLeo JA, et al. Cytokine and growth factor immunohistochemical spinal profiles in two animal models of mononeuropathy. Brain Res 1997;759:50-7.

12. DeLeo JA, et al. Transgenic expression of TNF by astrocytes increases mechanical allodynia in a mouse neuropathy model. Neuroreport 2000;11:599-602.

13. Fritsch EW, et al. The failed back surgery syndrome: reasons, intraoperative findings and long-term results. A report of 182 operative treatments. Spine 1996; 21:626-633.

14. Frymoyer, JW. Back Pain and Sciatica. N Engl J Med 1988;318:291300.

15. Genevay S, et al. Elevated levels of tumor necrosis factor-alpha in periradicular fat tissue in patients with radiculopathy from herniated disc. Spine 2008;33:2041-6.

16. George A, et al. Serial determination of tumor necrosis factor-alpha content in rat sciatic nerve after chronic constriction injury. Exp Neurol 1999;160:124-132.

17. Habtemariam $A$, et al. The role of mast cells in disc herniation inflammation. Spine 1999;24:1516-20. 
TNF-alpha levels in recurrent LDH patients correlate with chronic pain

18. Holmes GM, et al. Immunocytochemical localization of TNF type 1 and type 2 receptors in the rat spinal cord. Brain Res 2004;1025:210-9.

19. Kang JD, et al. Herniated lumbar intervertebral discs spontaneously produce matrix metalloproteinases, nitric oxide, interleukin-6, and prostaglandin E2. Spine 1996;21:271-7.

20. Koch A, et al. Nitric oxide and pro-inflammatory cytokines correlate with pain intensity in chronic pain patients. Inflamm Res 2007;56:32-7.

21. Lee HL, et al. Temporal expression of cytokines and their receptors mRNAs in a neuropathic pain model. Neuroreport 2004;15:2807-11.

22. Peul WC, et al. Surgery versus prolonged conservative treatment for sciatica. N Engl J Med 2007;356:2245-56.

23. Sacerdote $P$, et al. Transient early expression of TNF-alpha in sciatic nerve and dorsal root ganglia in a mouse model of painful peripheral neuropathy. Neurosci Lett 2008;436:210-3.

24. Sakuma $Y$, et al. Up-regulation of p55 TNF alpha-receptor in dorsal root ganglia neurons following lumbar facet joint injury in rats. Eur Spine J 2007; $16: 1273-1278$.

25. Schäfers $M$, et al. Selective stimulation of either tumor necrosis factor receptor differentially induces pain behavior in vivo and ectopic activity in sensory neurons in vitro. Neuroscience 2008;157:414-23.

26. Schäfers $M$, et al. Intramuscular injection of tumor necrosis factor-alpha induces muscle hyperalgesia in rats. Pain 2003a;104:579-88.

27. Schäfers $M$, et al. Tumor necrosis factor-alpha induces mechanical allodynia after spinal nerve ligation by activation of p38 MAPK in primary sensory neurons. J Neurosci 2003b;23:2517-21.

28. Stafford MA, et al. Sciatica: a review of history, epidemiology, pathogenesis, and the role of epidural steroid injection in management. Br J Anaesth 2007;99:461-73.

29. Takahashi $\mathrm{H}$, et al. Inflammatory cytokines in the herniated disc of the lumbar spine. Spine 1996;21:218-24.

30. Üçeyler $\mathrm{N}$, et al. Differential expression of cytokines in painful and painless neuropathies. Neurology 2007;69:42-49.

31. Valat JP, et al. Sciatica. Best Pract Res Clin Rheumatol 2010;24:24152.

32. Virri J, et al. Concomitant immunocytochemical study of macrophage cells and blood vessels in disc herniation tissue. Eur Spine $\mathrm{J}$ 1994;3:336-41. 
33. Xu JT, et al. The role of tumor necrosis factor-alpha in the neuropathic pain induced by Lumbar 5 ventral root transection in rat. Pain 2006;123:306-21.

34. Yang $L$, et al. Target depletion of distinct tumor necrosis factor receptor subtypes reveals hippocampal neuron death and survival through different signal transduction pathways. J Neurosci 2002;22:3025-32.

35. Youn $\mathrm{DH}$, et al. Exogenous tumor necrosis factor-alpha rapidly alters synaptic and sensory transmission in the adult rat spinal cord dorsal horn. J Neurosci Res 2008;86:2867-75.

36. Zhang L, et al. TNF-alpha contributes to spinal cord synaptic plasticity and inflammatory pain: Distinct role of TNF receptor subtypes 1 and 2 . Pain 2011;152:419-427. 


\section{Chapter}

The thalidomide analgesic effect is associated with differential TNF- $\alpha$ receptor expression in the dorsal horn of the spinal cord as studied in a rat model of neuropathic pain

Pablo Andrade, Veerle Visser-Vandewalle, John S. Del Rosario,

Marc A. Daemen, Wim A. Buurman, Harry W.M. Steinbusch, Govert Hoogland 


\section{Chapter 7}

\section{Abstract}

The proinflammatory cytokine tumor necrosis factor- $\alpha$ (TNF- $\alpha$ ) is well recognized as key player in nociceptive signaling. Yet, therapeutic capitalization of this knowledge requires a better understanding of how TNF receptors (TNFR) contribute to pain. To address this question, we studied TNFR expression in the chronic sciatic nerve constriction ( $\mathrm{CCl}$ ) model of neuropathic pain. $\mathrm{CCl}$ and sham operated rats received two subcutaneous injections (one immediately after surgery, the other on postoperative day 5) containing either saline, GABA-reuptake inhibitor (NO-711), insulin-like growth factor-1 (IGF-1), ZVAD or thalidomide. Mechanical (using von Frey filaments) and thermal hypersensitivity (Hargreaves test) were assessed preoperatively and weekly during the first four postoperative weeks. Spinal cord dorsal horn samples were collected from animals that were sacrificed at two weeks and four weeks after surgery, and analyzed for TNFR1 and TNFR2 mRNA levels by qPCR and protein levels by Western blot. Compared to saline, all applied drug treatments resulted in a faster recovery from mechanical and thermal hypersensitivity, yet in a potency order of thalidomide $>Z V A D=\mid G F-1>N O-711 . \mathrm{CCl}$ resulted in increased TNFR1 and TNFR2 mRNA and protein levels in the ipsilateral dorsal horn. Thalidomide was the only treatment that attenuated these increases. Finally, animals that showed a poor behavioral recovery were characterized by a significantly higher TNFR1/TNFR2 mRNA ratio. These data show that differential expression of TNFR in the dorsal horn is associated with recovery from pain in this model and suggest that the analgesic effects of thalidomide may act via this mechanism. 


\section{Introduction}

Neuropathic pain (NP) is an assortment of heterogeneous chronic conditions that are characterized by hyperpathia, hyperalgesia and allodynia. These disorders can be caused by a lesion or dysfunction of one or various segments in the somatosensory nervous system resulting in maladaptive signaling that is finally interpreted as pain (Baron et al., 2010; Jensen et al., 2011). Clinically, they manifest as focal, multifocal or generalized lesions in the peripheral and/or central nervous system (Baron, 2006). From a pathophysiological standpoint, the mechanism that underlies the chronicity of the disease and the location in terms of being related to the peripheral or the central nervous systems is still controversial. However, several lines of clinical and experimental evidence indicate that neuroinflammation is an important factor in the process of central sensitization (DeLeo et al., 1997; Koch et al., 2007; Schäfers et al., 2003; Üçeyler et al., 2007). Particularly the expression of proinflammatory cytokines in the dorsal root ganglion and dorsal horn $(\mathrm{DH})$ of the spinal cord has been largely shown to be implicated in the pathogenesis of NP. In this respect, the proinflammatory, immunoregulatory and nociceptive properties (neural hyperexcitability in the spinal cord) of tumor necrosis factor- $\alpha$ (TNF- $\alpha$ ) have received much attention in contrast to its receptors. The involvement of TNF- $\alpha$ in several inflammatory and NP models as a key player in the generation of nociceptive signals has been well documented (DeLeo et al., 2000; George et al., 1999; Sacerdote et al., 2008; Schafers et al., 2003; Xu et al., 2006; Youn et al., 2008). However, the participation of its receptors in regulating inflammation and NP is not yet fully understood.

TNF- $\alpha$ elicits its effects through its receptors type 1 (TNFR1) and type 2 (TNFR2). TNFR1 is expressed in almost all cells and is activated by membranebound and soluble trimeric forms of TNF, whereas TNFR2 is found in restricted cell types and respond to the membrane-bound form of the TNF homotrimer (Grell et al., 1995). Upon activation TNFR1 bind to tumor necrosis factor receptor type 1-associated DEATH domain and Fas-associated protein with death domain, ultimately resulting in apoptosis, while activated TNFR2 mostly activate tumor necrosis factor receptor-associated factor 2 and nuclear factor$\mathrm{KB}$, and ultimately result in cell survival, proliferation, and an inflammatory response (for review see (Aggarwal, 2003; Andrade et al., 2011a; Faustman and Davis, 2010)). Recent findings suggest that both receptors play different roles in the development of NP (Constantin et al., 2008; Schäfers et al., 2008). Nevertheless, it remains unclear how the different intracellular responses to TNFR1 and 2 activation are affected by anti-inflammatory therapies for NP in 


\section{Chapter 7}

general, and anti-TNF therapies in particular. In order to differentiate the involvement of TNF- $\alpha$ and its receptors in preventing nociception, we addressed three different systems that have been implicated in pain modulation. Firstly, inhibiting the inflammatory process generated by TNF- $\alpha$ with thalidomide (Sampaio et al., 1991). Secondly, approaching the viability and survival of the cell exposed to inflammation through broad inhibition of apoptosis with the anti-caspase agent ZVAD (Scholz et al., 2005), and through general proliferation induction with IGF-1 (Zhang et al., 2006). Finally, a noninflammatory mechanism of antinociception modulated through spinal cord inhibition with the GABA reuptake inhibitor NO-711 (Daemen et al., 2008). The purpose of this study was to investigate if the analgesic effect of antiinflammatory drugs is accompanied by changes in the spinal cord TNFR levels.

\section{Experimental Procedure}

\subsection{Animals and experimental groups}

All experiments were approved by the Animal Experiments and Ethics Committee of Maastricht University and followed the ethical guidelines established by the International Association of the Study of Pain (IASP) for animal research (Zimmermann, 1983). Adult male Lewis rats (Charles River Laboratories, France) weighing 200-250g were used in all experiments. Animals were housed individually in standard cages using sawdust bedding with free access to food and water in a humidity and temperature controlled room with lights on from 7:00 till 19:00hrs and a radio. The animals were allowed to habituate to the housing environment 1 week prior to surgery. Rats were randomly assigned to undergo a sham $(n=20)$ or $\mathrm{CCl}$ procedure $(n=48)$, and to one of the following therapy groups: $0.9 \% \mathrm{NaCl}$ (saline control), NO-711 (1-[2[[(diphenylmethylene)imino]oxy]ethyl]-1,2,5,6-tetrahydro-3-pyridinecarboxylic acid hydrochloride), IGF-1 (insulin-like growth factor 1), ZVAD ((carbobenzoxyvalyl-alanyl-aspartyl-[O-methyl]- fluoromethylketone)) or thalidomide. The animals were allowed to acclimatize to the housing environment 1 week previous to the surgery. 


\subsection{Surgical procedures}

Animals were anesthetized using xylazine $(10 \mathrm{mg} / \mathrm{kg}$, s.c.) and ketamine (90 $\mathrm{mg} / \mathrm{kg}$, i.p.). After verifying the absence of nociceptive reflexes, the left hind limb was shaved and disinfected with povidone-iodine. A superficial skin incision and blunt dissection of the musculature was performed and the left sciatic nerve was exposed at the level of the mid-thigh. After this, in $\mathrm{CCl}$ cases, a microscope was used to tie four 3-0 chromic gut (SMI sutures, St. Vith, Belgium) ligatures loosely around the nerve leaving blood supply intact (Bennett and Xie, 1988). For the sham cases, the nerve was exposed in its trajectory without further manipulation. For both types of procedures, the muscular layers and skin were sutured using 3-0 Vycril (Ethicon, New Brunswick, NJ).

\subsection{Drug administration}

All drugs were obtained from Sigma-Aldrich (St. Louis, MO), dissolved in sterile $0.9 \% \mathrm{NaCl}$ to $0.5 \mathrm{ml}$ final volume, and administered by s.c. injection. Each animal received the same drug twice, i.e., once immediately after the surgical procedure (in the contralateral $\operatorname{limb}$ ) and once on postoperative day 5 (in the neck). Each injection contained (per kg bodyweight): $60 \mathrm{mg}$ NO-711 (Daemen et al., 2008), 0.1 mg IGF-1 (Bitar et al., 1996; Bluthe et al., 2006), 15 mg ZVAD (Li et al., 2000) or $50 \mathrm{mg}$ thalidomide (Choi et al., 2010).

\subsection{Behavioral testing}

Mechanical and thermal hypersensitivity were assessed one day before surgery and weekly during the first four postoperative weeks by an observer that was blind to the surgical procedure and treatment. Mechanical hypersensitivity was assessed using the von Frey filaments test by measuring the hindpaw withdrawal in response to stimuli. The animals were tested on both hindpaws using 14 filaments with gradual increasing stiffness $(0.16,0.4,0.6,1,1.4,2,4$, $6,8,10,15,26,60$ and $100 \mathrm{~g}$ ) (Stoelting Co, Wood Dale, IL). Animals were allowed 30 minutes of acclimatization to the wire mesh floor before the filaments were pushed against the mid-plantar surface until a mild curvature of the filament or a paw withdrawal response was observed. The filaments were tested in an increasing weight force order and each filament was tested 5 times before the next filament was applied. The withdrawal threshold was defined as 
the smallest filament to which a rat responded by lifting its paw in three out of five trials. Thermal hypersensitivity was measured using the Hargreaves test on both hind paws (Hargreaves et al., 1988). Following a 10 minute acclimatization period on the glass surface, the mid-plantar surface was exposed to an infrared heat source and the time to withdraw the limb was recorded (known as withdrawal latency). To avoid tissue damage, the heating source was programmed to stop after 20 seconds, and the maneuver was applied only once on the testing day.

\subsection{Tissue processing}

From each experimental group half of the number of animals was sacrificed at 2 weeks after surgery, and the other half at 4 weeks after surgery. Two hours after behavioral testing, rats were sedated by pentobarbital $(150 \mathrm{mg} / \mathrm{kg}$, i.p.) followed by a transcardial perfusion with $50 \mathrm{ml}$ ice-cold $0.9 \% \mathrm{NaCl}$. The spinal cord was obtained by flushing the spinal canal with $5 \mathrm{ml}$ ice-cold $0.9 \% \mathrm{NaCl}$. Next, the DH was collected by removing the meninges and dissecting the spinal cord at the L4-L6 level, axially dividing it in an ipsilateral and contralateral side and removing the ventral horn. Upon collection, samples were immediately frozen in liquid nitrogen and then stored at $-80^{\circ} \mathrm{C}$ until analysis by qPCR or Western blotting.

\subsection{Real-time qPCR}

RNA was isolated using TRIzol (Invitrogen, CA). Next, cDNA was generated from $1 \mu \mathrm{g}$ total RNA using a First Strand cDNA Synthesis Kit (Fermentas International Inc., Ontario, Canada) according to the manufacturer protocol. TNF- $\alpha$, TNFR1 and TNFR2 mRNA expression was measured by qPCR in a LightCycler 480 Real-Time PCR System using SYBR Green LightCycler 480 SYBR Green I Master (Roche Diagnostics) for 50 cycles of $94^{\circ} \mathrm{C} 30 \mathrm{~s} / 63^{\circ} \mathrm{C} 15 \mathrm{~s}$ $172^{\circ} \mathrm{C} 15 \mathrm{~s}$. Amplicons were generated using the following primer sets: TNFR1forward 5'- CTC TTG GTG ACC GGG AGA AG -3' and reverse 5'- GGT TCC TTT GTG GCA CTT GGT -3', and TNFR2-forward 5'- CAT CCC TGT GTC CTT GGG -3' and reverse 5'- CCC GTG ATG CTT GGT TCA -3'. Glyceraldehyde 3phosphate dehydrogenase (GAPDH) was used as reference gene generated by forward primer 5'- GCA AGA GAG AGG CCC TCA G -3' and reverse 5'- TGT GAG GGA GAT GCT CAG TG - 3 '. Melting curve analysis and $2 \%$ agarose gel 
were performed to verify product specificity. Quantification was performed with the delta CT method and normalized to the reference gene. In each animal the value of the ipsilateral $\mathrm{DH}$ was expressed as fold increase over the value of the contralateral $\mathrm{DH}$.

\subsection{Western blot}

Samples were homogenized in ice-cold lysis buffer (phosphate buffered saline (PBS) containing 1\% Igepal, 0.1\% Triton X-100, 1 mM EDTA, $1 \mathrm{mM}$ EGTA, and COMPLETE protease inhibitor cocktail (Roche Diagnostics, IN)). Proteins $(20 \mu \mathrm{g})$ were separated by SDS-10\% polyacrylamide gel electrophoresis for $1.5 \mathrm{~h}$ at $100 \mathrm{~V}$ and then transferred to a pure nitrocellulose membrane (Bio-Rad Laboratories, CA) for $1 \mathrm{~h}$ at $100 \mathrm{~V}$. Next, membranes were rinsed with PBS, blocked in Odyssey blocking buffer (LI-COR Biosciences, NE) 1:2 diluted in PBS for 1 hour and overnight incubated at $4^{\circ} \mathrm{C}$ with primary antibody (diluted in 1:1 PBS:blocking buffer). Primary antibodies used included: monoclonal mouseanti rat TNF- $\alpha$ (1:500; 31Y-2, sc-80383, Santa Cruz Biotechnology, CA), polyclonal rabbit-anti rat TNF-R1 (1:500; H-271, sc-7895, Santa Cruz Biotechnology), polyclonal rabbit-anti rat TNF-R2 (1:500; H-202, sc-7862, Santa Cruz Biotechnology), and monoclonal mouse-anti rat $\beta$-Actin (1:500; ACTBD11B7, sc-81178, Santa Cruz Biotechnology). Subsequently, membranes were rinsed in PBS, incubated for $1 \mathrm{~h}$ with 1:10.000 Alexa Fluor 680 donkey anti-mouse IgG or 1:10.000 Alexa Fluor 800 goat anti-rabbit IgG (Molecular Probes, OR), rinsed with PBS, and visualized by an enhanced chemiluminescence detection Odyssey Infrared Imaging System (LI-COR Biosciences). Immunoreactive bands were quantified by calculating the optical density ratio of the band of interest over that of $\beta$-Actin using Image $1.42 q$ software (National Institute of Health, MD).

\subsection{Statistical analysis}

Group data were expressed as mean \pm SEM and analyzed by one-way analysis of variance (ANOVA) using GraphPad Prism 4 software (San Diego, CA) for $\mathrm{CCl}$ animals to analyze differences between drug treatments. A post-hoc Bonferroni test for multiple comparisons was used to investigate differences with the $\mathrm{NaCl}$ injected group. Comparison between two groups was analyzed by a Student's $t$-test. P-values $<0.05$ were considered significant. 


\section{Chapter 7}

\section{Results}

\subsection{Behavioral Testing}

Presurgically, animals withdrew both hind limbs from the mechanical stimuli when a stiffness force of about $100 \mathrm{~g}$ was applied and had a withdrawal latency to heat of about 14s. The contralateral hind paw of sham and $\mathrm{CCl}$ operated rats reacted similarly during the four postsurgical weeks. Mechanical hypersensitivity was observed in the ipsilateral hind paw during the four postoperative weeks in $\mathrm{CCl}$, but not in sham operated animals (fig. 1A). Yet, two weeks after $\mathrm{CCl}$ surgery, thalidomide treated rats show a significantly higher withdrawal threshold $(55.2 \pm 4.8 \mathrm{~g})$ than saline treated rats $(25.6 \pm 0.8 \mathrm{~g})$. At three weeks after surgery this threshold had further increased $(63.6 \pm 3.6 \mathrm{~g})$ and also ZVAD and IGF-1 treated animals showed a significantly improved withdrawal threshold (57.3 $\pm 3.7 \mathrm{~g}$ and $54.1 \pm 3.2 \mathrm{~g}$, respectively) than saline treated rats that still showed a similar withdrawal latency $(25.8 \pm 0.5 \mathrm{~g})$. Finally, at four weeks after surgery, saline treated $\mathrm{CCl}$ animals start to recover and displayed a withdrawal threshold of $40.3 \pm 1.4 \mathrm{~g}$ ). At this time point, thalidomide treated rats still showed a significantly improved withdrawal threshold of $72.8 \pm$ $5.2 \mathrm{~g}$.

Thermal hypersensitivity was observed in the ipsilateral hind paw during the four postoperative weeks in $\mathrm{CCl}$, but not in sham operated animals (fig. 1B). The effect of the different treatments on recovery from thermal hypersensitivity was similar to that on mechanical hypersensitivity. In other words, at one week after $\mathrm{CCl}$ surgery thalidomide treated rats showed a significantly longer withdrawal latency than saline treated rats. At two weeks after surgery this latency further increased and also ZVAD treated animals showed a significantly longer withdrawal latency than saline treated rats. At three weeks after surgery these latencies had increased even more and also IGF-1 treated animals showed a significantly longer withdrawal latency. Finally, at four weeks after surgery thalidomide, ZVAD and IGF-1 still showed a significantly better recovery to thermal hypersensitivity than saline treated rats. NO-711 treatment had no effect on recovery from $\mathrm{CCl}$-induced mechanical or thermal hypersensitivity. 
A

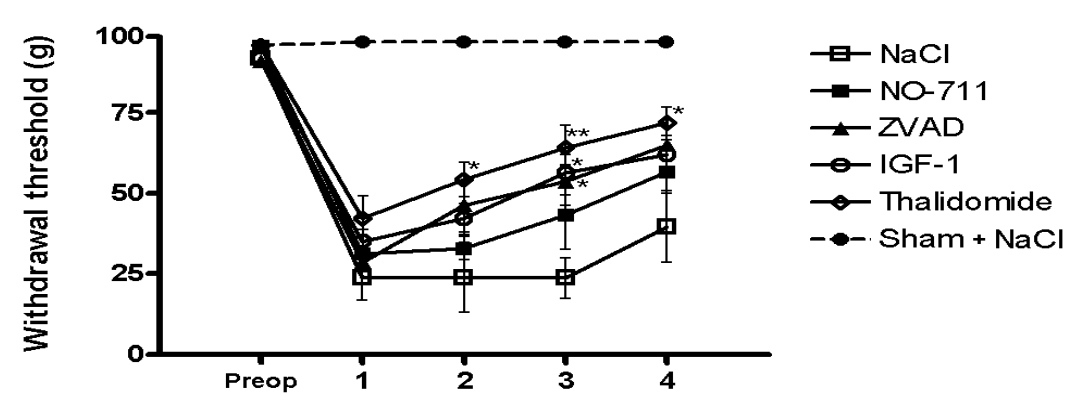

Postoperative time (w)

B

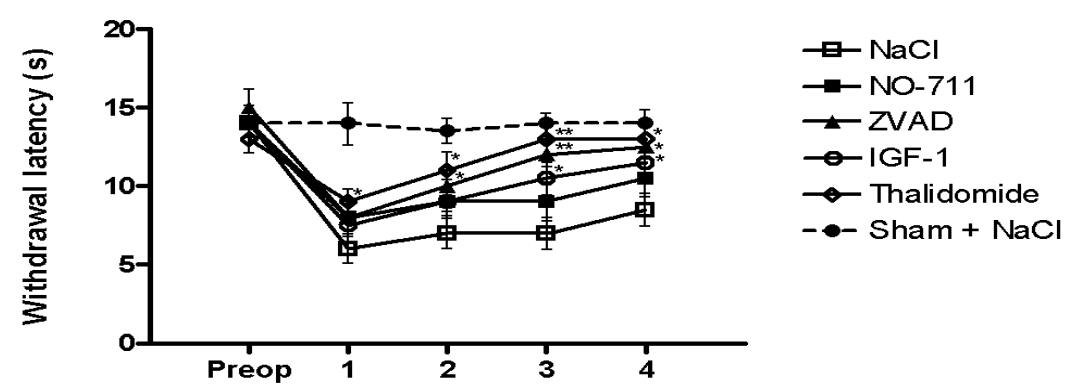

Postoperative time (w)

Figure 1. Thalidomide potently accelerates recovery from mechanical and thermal hypersensitivity. (A) Mechanical hypersensitivity was assessed preoperatively and weekly during the first 4 weeks following $\mathrm{CCl}$ by applying von Frey filaments. (B) Thermal hypersensitivity was assessed preoperatively and weekly during the first 4 weeks following $\mathrm{CCl}$ by applying an infrared heat source to the plantar surface of the ipsilateral hind paw (Hargreaves test). Only ipsilateral hind paw data of $\mathrm{CCl}$ rats are shown with the continuous lines pattern. Ipsilateral hind paw data of saline treated sham animals is represented with the dotted line pattern. ANOVA test analysis showed statistical differences between $\mathrm{CCl}$ operated animals from postoperative week 2 to week 4 for mechanical hypersensitivity $(A)$ and from postoperative week 1 to week 4 for thermal hyperalgesia $(B)$; sham operated animals were not included for this analysis. Data are expressed as mean \pm SEM of observations from 4-10 animals. ${ }^{*} p<0.05$ and ${ }^{* *} p<0.01$ compared to $\mathrm{NaCl}$ injected rats (post-hoc Bonferroni test). $g=$ grams, $w=$ week, $s=$ seconds. 


\subsection{TNF- $\alpha$ receptors mRNA expression}

TNFR1 and TNFR2 mRNA were undetectable in dorsal horn samples from sham animals. TNFR1 mRNA expression at 2 and 4 weeks after CCl surgery in saline controls was comparable between both time points (fig. $2 A$ ). In these animals the ipsilateral DH expressed 5-6 times more TNFR1 mRNA than the contralateral DH. Whereas NO-711, ZVAD and IGF-1 treated rats showed similar TNFR1 mRNA levels as saline controls, and thalidomide treated rats expressed significantly less TNFR1 mRNA.

TNFR2 mRNA expression at 2 and 4 weeks after $\mathrm{CCl}$ surgery in saline controls was comparable between both time points (fig. 2B). In these animals the ipsilateral DH expressed 3-4 times more TNFR2 mRNA than the contralateral side. A treatment effect was observed only for thalidomide, i.e., at 2 weeks after surgery there was a significantly reduced TNFR2 expression compared to saline controls. Compared to this reduced expression at 2 weeks, samples collected at 4 weeks showed a significantly increased TNFR2 level.

To investigate if mechanical or thermal hypersensitivity were accompanied by an individual altered TNFR expression, we compared the TNFR1 / TNFR2 mRNA ratio of poor recovering (i.e. a mechanical withdrawal threshold $<50 \%$ of the presurgical value or a heat withdrawal latency $<50 \%$ of the presurgical value) with that of good recovering (i.e. a mechanical withdrawal threshold $\geq$ $50 \%$ of the presurgical value or a heat withdrawal latency $\geq 50 \%$ of the presurgical value) $\mathrm{CCl}$ animals. The percentage of recovery was estimated by setting the behavioral test baseline value of each rat as the maximum score $(100 \%)$, after that the values obtained in further evaluations ( 2 and 4 weeks postoperatively) were calculated according the individual initial maximum score as a percentage value. This analysis learned that animals that poorly recover from mechanical hypersensitivity are characterized by a significantly increased TNFR1 / TNFR2 ratio at 2 weeks postoperatively (fig. 2C) and that poor recovery from thermal hypersensitivity is accompanied by increased TNFR1 / TNFR2 ratio's at 2 and 4 weeks postoperatively (fig. 2D). Melting curve and $2 \%$ agarose gel analysis verified the specificity of all products tested for mRNA expression. 


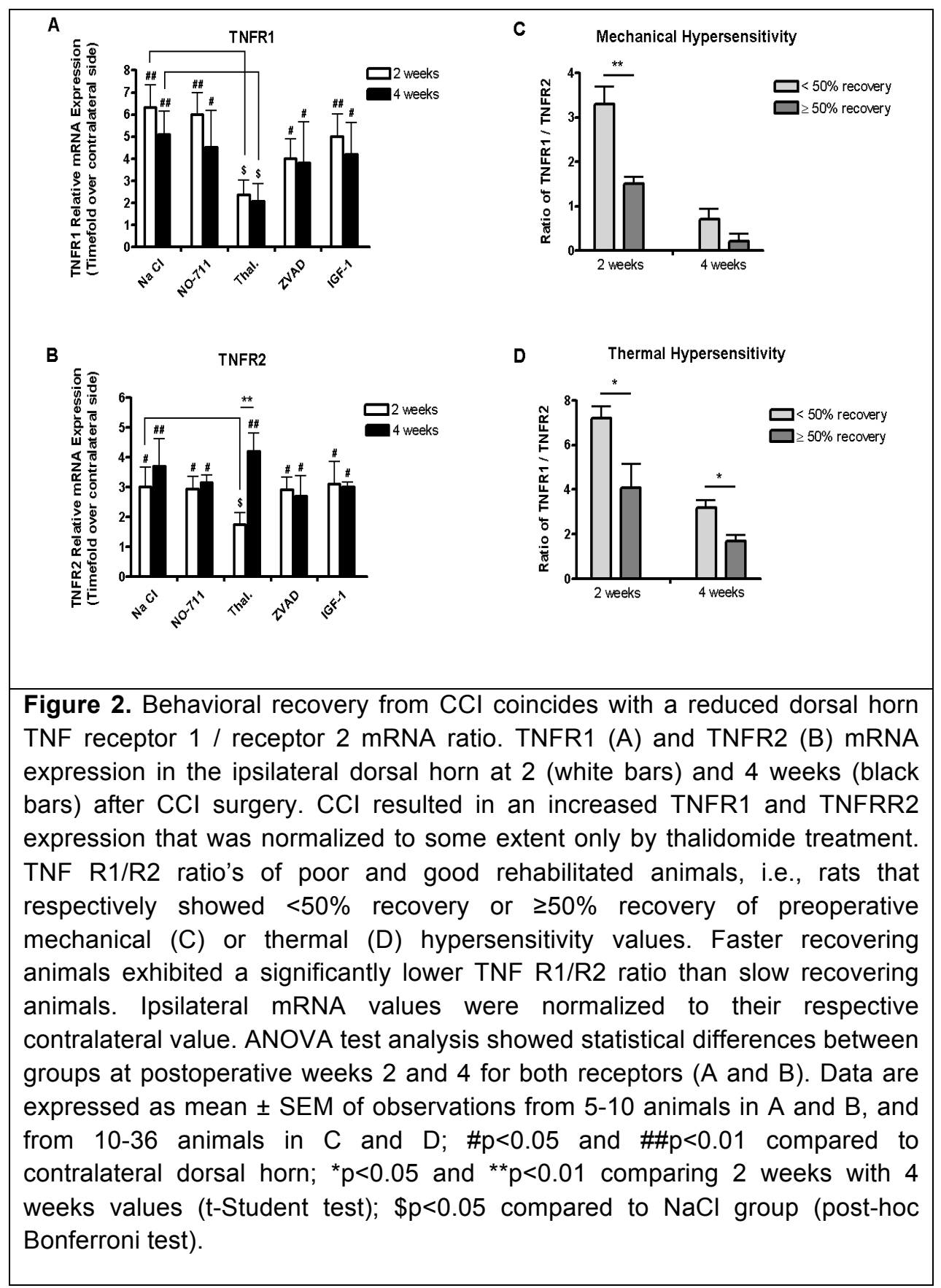




\subsection{TNF- $\alpha$ and TNF- $\alpha$ receptors protein expression}

By Western blotting DH samples from sham animals, we only detected TNFR1. Also contralateral $\mathrm{DH}$ samples from $\mathrm{CCl}$ animals expressed only TNFR1 up to a comparable level as that in sham animals (data not shown). In contrast, ipsilateral $\mathrm{DH}$ samples from $\mathrm{CCl}$ animals collected at 2 and 4 weeks after surgery expressed TNFR1 (55 kD; fig. 3A), TNFR2 (75 kD; fig. 3B) and membranous TNF- $\alpha$ (mTNF- $\alpha$; $26 \mathrm{kD}$; fig. $3 \mathrm{C}$ ). Only thalidomide treated rats expressed altered protein levels, i.e., compared to saline controls was TNFR1 reduced by about $50 \%$ at 2 weeks (fig. 3D), was TNFR2 unchanged (fig. 3E), and was mTNF- $\alpha$ reduced by about $40 \%$ at 2 and 4 weeks (fig. 3F). Finally, within the $\mathrm{CCl}$ thalidomide treatment group at 4 weeks, postoperative samples expressed significantly higher TNFR2 levels than 2 weeks postoperative samples (fig. 3E).

\section{Discussion}

The present data corroborate the statement that thalidomide is a potent inhibitor of TNF- $\alpha$ of early and late stages of inflammation secondary to peripheral nerve injury (Choi et al., 2010; Gu et al., 2010). Thalidomide administration also strongly blocked TNFR1 mRNA and protein expression on both early and late stages, whereas TNFR2 levels were somewhat downregulated in the early stage and upregulated in the late stage. Furthermore, western blot analysis showed that only thalidomide treated $\mathrm{CCl}$ rats showed significant reduction of TNF- $\alpha$ and TNFR1 levels. In addition, we observed an increased TNFR1 / TNFR2 mRNA ratio in animals with poor behavioral recovery. These findings suggest that thalidomide reduces mechanical and thermal hypersensitivity primarily through downregulation of mTNF- $\alpha$ and TNFR1 in the spinal cord, and to a lesser extent by changes in TNFR2 expression.

Previous preclinical studies demonstrated an association between elevated TNF- $\alpha$ levels and pain behavior (Bianchi et al., 2007; Covey et al., 2002; DeLeo et al., 2000; Schäfers et al., 2003). In line with these findings, we found that increased pain behavior after $\mathrm{CCl}$ coincided with an increased TNF- $\alpha$ and TNFR1 expression. Interestingly, thalidomide treatment reduced pain behavior and TNF- $\alpha$ and TNFR1 expression. In addition, no TNF- $\alpha$ and low levels of TNFR1 were detected in the contralateral dorsal horn of $\mathrm{CCl}$ rats, where no painful reactions were elicited after paw stimulation. These findings are in line with studies that performed a surgical intervention without sciatic nerve ligation 
and also were unable to detect TNF- $\alpha$ in nervous tissue (Arandjelovic et al., 2007; Costa et al., 2007; Covey et al., 2002; George et al., 1999). However, in contrast with these results, some reports suggest that bilateral hypersensitivity and TNF- $\alpha$ expression can be present in equivalent lesion models, mainly in the early phases (Dubový et al., 2006; Hatashita et al., 2008). The latter reports data could be attributed to the extent of the lesion that induces a generalized inflammatory response, or to the crossing of fibers inside the spinal cord.

With respect to changes in TNF- $\alpha$ receptors, our data suggest that recovery from pain depends on the balance between TNFR1 and TNFR2. Whereas increased TNFR1 levels were associated with elevated pain intensity, animals with considerable recovery levels showed higher TNFR2 expression and more balanced ratios between the receptors. Similarly, other studies have shown that over expression, exogenous administration or selective stimulation of TNFR1 results in increased neuronal excitation and pain (Andrade et al., 2011b; Schäfers et al., 2008; Zhang et al., 2011). However, it is important to note that in all these cases TNFR2 was also required to elicit pain behavior, particularly in the early stage of the inflammatory process (Zhang et al., 2011). Moreover, the combination of both receptors has been shown to produce more and higher pain responses in rodents than when only TNFR1 was stimulated (Schäfers et al., 2008). Interestingly, TNFR2 protein was not detectable in the ipsilateral DH of sham controls. As noted previously, TNFR2 is only expressed in nervous and connective tissue after peripheral injury (Andrade et al., 2011b; Holmes et al., 2004), suggesting that expression of this receptor requires an inducer (Holmes et al., 2004; Vandenabeele et al., 1995). However, here we observed very low TNFR2 mRNA levels in the contralateral $\mathrm{DH}$ of $\mathrm{CCl}$ animals, and in the contralateral and ipsilateral DH of sham animals. Yet, TNFR2 protein was not detected in any of these samples. These findings suggest that the protein expression of TNFR requires a certain threshold of mRNA expression. Alternatively, TNFR2 protein degradation seems unlikely since it was clearly detectable in the ipsilateral $\mathrm{DH}$ of $\mathrm{CCl}$ animals. In addition, we never observed lower molecular weight breakdown products in the blots. These observations may have further implications for pain treatment by mRNA-targeting. 


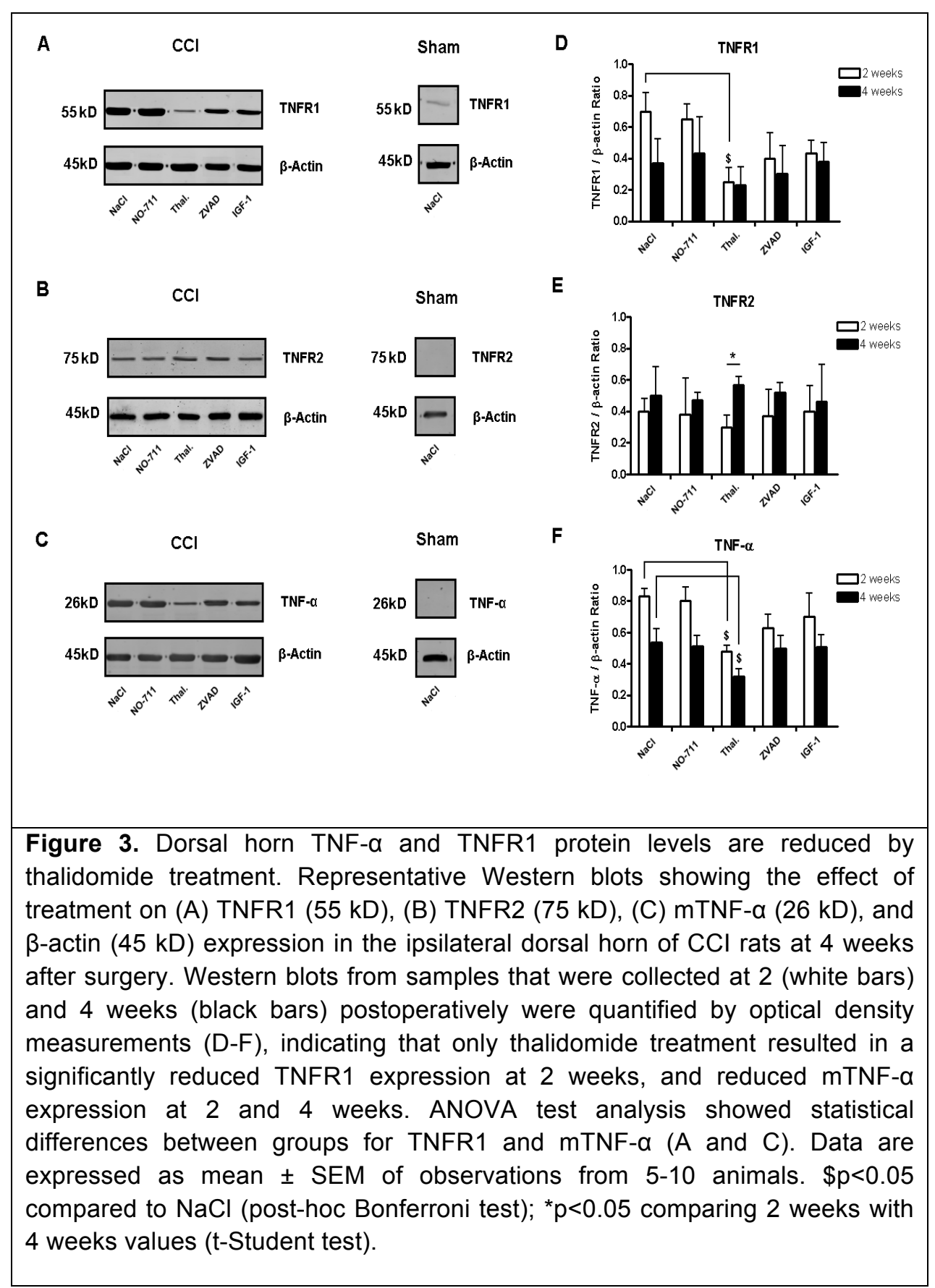


The antialgesic effects of thalidomide was also shown in other models of peripheral nervous system lesion, inflammatory pain and bone cancer pain (Choi et al., 2010; Gu et al., 2010; He et al., 2010; Ribeiro et al., 2000; Sommer et al., 1998). So far, the suggested mechanism for this effect is based on the selective capacity of thalidomide to inhibit the synthesis of TNF- $\alpha$ (Moreira et al., 1993; Sampaio et al., 1991). Nonetheless, it is not completely clear if thalidomide also affects the expression of TNF- $\alpha$ receptors or if it does it indirectly by reducing the levels of TNF- $\alpha$. This may be relevant for its therapeutic application since in recent years these receptors have been identified as key players in nociception (George et al., 2005; Holmes et al., 2004; Lee et al., 2004; Sakuma et al., 2007). Based on our data, from a spatial point of view, it is hard to claim exactly were the effects of the drugs are taking place. However, cellularly we know which mechanism is the most involved to generate chronic pain. Because we used different mechanisms of action by applying different drugs, we know that the most important action to reduce pain is by blocking the anterograde transport of TNF- $\alpha$ (Schäfers et al., 2002). In the current study, an observation that supports a correlation between TNF- $\alpha$ and TNF- $\alpha$ receptors expression and pain severity is the lack of efficacy to reduce pain scores showed by NO-711, ZVAD and IGF-1. These findings are remarkable since some of them affect the inflammatory cascade at different levels, but not selectively for TNF- $\alpha$, suggesting the critical role of this cytokine and its receptors in the generation of pain. It is important to mention that ZVAD is a potent general caspase inhibitor, therefore its action can be considered to take place downstream from TNF- $\alpha$ receptor stimulation (Scholz et al., 2005). Also, ZVAD does not have intrinsic analgesic properties; however it has proven to reduce pain hypersensitivity (Scholz et al., 2005). Thus, inhibition of caspases may reduce apoptosis and perhaps consequently reduce pain sensitivity. However, we found that upregulated TNFR1 expression is enough to increase pain sensitivity. This study is not the first report that suggests that caspase inhibition is not sufficient to prevent cytotoxicity (Davies et al., 2010), even when marked caspase activation has been reported in these models ( $\mathrm{Di}$ Cesare Mannelli et al., 2009). The mild effect of IGF-1 on pain sensitivity is also noteworthy, because it functions mainly as a stimulator of proliferation and an apoptosis inhibitor (Gao et al., 1999). Specially because it has been previously described as an antinociceptive agent when it was administered intrathecally or directly into the ventricles, and as an effective blocker of TNFR1 (Bitar et al., 1996; Bluthe et al., 2006; Palin et al., 2007). The lack of results from this drug possibly suggests that the viability of the cell is more dependant on the inhibition of apoptotic pathways, and/or that peripheral administration was not 


\section{Chapter 7}

enough to block TNF- $\alpha$ action, even when one previous study reported that intraperitoneal administration was sufficient (Johnson et al., 2005). With respect to NO-711, this drug functions as an inhibitor of the GABA transporter-1 and consequently increases the GABAergic activity in the dorsal horn (Daemen et al., 2008). Therefore, the reduced action of this drug in our study could suggest that increasing GABAergic transmission in the spinal cord is not enough to counteract glutamate excitotoxicity after peripheral nerve injury, particularly after an increased inflammatory response. These data are in line with previous findings showing that no significant quantitative GABAergic changes were found in the spinal cord after peripheral lesion (Lee et al., 2010); however, in that study the behavioral tests showed an improvement. In summary, the modulation of inflammation via TNF- $\alpha$ (thalidomide) have shown better antinociceptive effects than those obtained with apoptosis inhibition (ZVAD), proliferation induction (IGF-1) or interneuron spinal inhibition (NO-711).

Taken together, the current study provides evidence that inflammation (mTNF- $\alpha$ and TNFR1) is closely involved in pain-related behavior. In fact, it may appear that the modulation of TNF- $\alpha$ receptors could have a larger role in preventing nociception than through general inhibition of apoptosis, spinal inhibition or broad proliferation induction. Thalidomide provided statistically significant reductions in pain behavior, most probably due to the inhibition of mTNF- $\alpha$ and the downregulation of one of its receptors, TNFR1.

In conclusion, the results of the present study indicate that thalidomide reduces mechanical and thermal hypersensitivity through the downregulation of mTNF- $\alpha$ and TNFR1, but not TNFR2, in the spinal cord. The increased ratio of TNFR1 over TNFR2 in rats with poor outcome may give us insights on the pathogenesis of chronic pain secondary to peripheral damage. Therapeutic TNFR2 upregulation or TNFR1 inhibition after mTNF- $\alpha$ release could decrease nociceptive signaling after established nervous lesions.

\section{References}

1. Aggarwal BB, et al. Signalling pathways of the TNF superfamily: a double-edged sword. Nat Rev Immunol 2003;3:745-56.

2. Andrade $P$, et al. Role of TNF-alpha during central sensitization in preclinical studies. Neurol Sci 2011a;32:757-771.

3. Andrade $P$, et al. Tumor necrosis factor-alpha levels correlate with postoperative pain severity in lumbar disc hernia patients: Opposite 
clinical effects between tumor necrosis factor receptor 1 and 2. Pain 2011b;152:2645-2652.

4. Arandjelovic $S$, et al. A derivative of the plasma protease inhibitor alpha(2)-macroglobulin regulates the response to peripheral nerve injury. J Neurochem 2007;103:694-705.

5. Baron R, Mechanisms of disease: neuropathic pain--a clinical perspective. Nat Clin Pract Neurol 2006;2:95-106.

6. Baron R, et al. Neuropathic pain: diagnosis, pathophysiological mechanisms, and treatment. Lancet Neurol 2010;9:807-19.

7. Bennett GJ, Xie YK. A peripheral mononeuropathy in rat that produces disorders of pain sensation like those seen in man. Pain 1988;33:87107.

8. Bianchi $M$, et al. Increased tumor necrosis factor-alpha and prostaglandin E2 concentrations in the cerebrospinal fluid of rats with inflammatory hyperalgesia: the effects of analgesic drugs. Anesth Analg 2007;104:949-54.

9. Bitar MS, et al. Antinociceptive action of intrathecally administered IGF-I and the expression of its receptor in rat spinal cord. Brain Res 1996;737:292-4.

10. Bluthe RM, et al. Effects of insulin-like growth factor-I on cytokineinduced sickness behavior in mice. Brain Behav Immun 2006;20:57-63.

11. Choi Jl, et al. Antiallodynic Effect of Thalidomide and Morphine on Rat Spinal Nerve Ligation-induced Neuropathic Pain. Korean J Pain 2010;23:172-8.

12. Constantin CE, et al. Endogenous tumor necrosis factor alpha (TNFalpha) requires TNF receptor type 2 to generate heat hyperalgesia in a mouse cancer model. J Neurosci 2008;28:5072-81.

13. Costa B, et al. The non-psychoactive cannabis constituent cannabidiol is an orally effective therapeutic agent in rat chronic inflammatory and neuropathic pain. Eur J Pharmacol 2007;556:75-83.

14. Covey WC, et al. Expression of neuron-associated tumor necrosis factor alpha in the brain is increased during persistent pain. Reg Anesth Pain Med 2002;27:357-66.

15. Daemen MA, et al. Upregulation of the GABA-transporter GAT-1 in the spinal cord contributes to pain behaviour in experimental neuropathy. Neurosci Lett 2008;444:112-5.

16. Davies JW, et al. Pharmacology of capsaicin-, anandamide-, and Narachidonoyl-dopamine-evoked cell death in a homogeneous transient 
receptor potential vanilloid subtype 1 receptor population. $\mathrm{Br} \mathrm{J}$ Anaesth 2010;104:596-602.

17. DeLeo JA, et al. Cytokine and growth factor immunohistochemical spinal profiles in two animal models of mononeuropathy. Brain Res 1997;759:50-7.

18. DeLeo JA, et al. Transgenic expression of TNF by astrocytes increases mechanical allodynia in a mouse neuropathy model. Neuroreport 2000;11:599-602.

19. Di Cesare Mannelli L, et al. Neuropathy-induced apoptosis: protective effect of physostigmine. J Neurosci Res 2009;87:1871-6.

20. Dubový $P$, et al. Intra- and extraneuronal changes of immunofluorescence staining for TNF-alpha and TNFR1 in the dorsal root ganglia of rat peripheral neuropathic pain models. Cell Mol Neurobiol 2006;26:1205-17.

21. Faustman D, Davis M. TNF receptor 2 pathway: drug target for autoimmune diseases. Nat Rev Drug Discov 2010;9:482-93.

22. Gao WQ, et al. IGF-I deficient mice show reduced peripheral nerve conduction velocities and decreased axonal diameters and respond to exogenous IGF-I treatment. J Neurobiol 1999;39:142-52.

23. George A, et al. Tumor necrosis factor receptor 1 and 2 proteins are differentially regulated during Wallerian degeneration of mouse sciatic nerve. Exp Neurol 2005;192:163-6.

24. George A, et al. Serial determination of tumor necrosis factor-alpha content in rat sciatic nerve after chronic constriction injury. Exp Neurol 1999;160:124-32.

25. Grell M. The transmembrane form of tumor necrosis factor is the prime activating ligand of the $80 \mathrm{kDa}$ tumor necrosis factor receptor. Cell 1995;83:793-802.

26. $\mathrm{Gu} \mathrm{X}$, et al. Intraperitoneal injection of thalidomide attenuates bone cancer pain and decreases spinal tumor necrosis factor-alpha expression in a mouse model. Mol Pain 2010;6:64.

27. Hargreaves $K$, et al. A new and sensitive method for measuring thermal nociception in cutaneous hyperalgesia. Pain 1988;32:77-88.

28. Hatashita $S$, et al. Contralateral neuropathic pain and neuropathology in dorsal root ganglion and spinal cord following hemilateral nerve injury in rats. Spine 2008;33:1344-51.

29. He $\mathrm{XH}$, et al. TNF-alpha contributes to up-regulation of Nav1.3 and Nav1.8 in DRG neurons following motor fiber injury. Pain 2010;151:266-79. 
30. Holmes GM, et al. Immunocytochemical localization of TNF type 1 and type 2 receptors in the rat spinal cord. Brain Res 2004;1025:210-9.

31. Jensen TS, et al. A new definition of neuropathic pain. Pain 2011;152:2204-2205.

32. Johnson DR, et al. Inhibition of vagally mediated immune-to-brain signaling by vanadyl sulfate speeds recovery from sickness. Proc Natl Acad Sci USA 2005;102:15184-9.

33. Koch A, et al. Nitric oxide and pro-inflammatory cytokines correlate with pain intensity in chronic pain patients. Inflamm Res 2007;56:32-7.

34. Lee HL, et al. Temporal expression of cytokines and their receptors mRNAs in a neuropathic pain model. Neuroreport 2004;15:2807-11.

35. Lee J, et al. Are spinal GABAergic elements related to the manifestation of neuropathic pain in rat? Korean J Physiol Pharmacol 2010;14:59-69.

36. Li M, et al. Functional role of caspase-1 and caspase-3 in an ALS transgenic mouse model. Science 2000;288:335-9.

37. Moreira $A L$, et al. Thalidomide exerts its inhibitory action on tumor necrosis factor alpha by enhancing mRNA degradation. J Exp Med 1993;177:1675-80.

38. Palin $\mathrm{K}$, et al. TNFalpha-induced sickness behavior in mice with functional $55 \mathrm{kD}$ TNF receptors is blocked by central IGF-I. J Neuroimmunol 2007;187:55-60.

39. Ribeiro RA, et al. Analgesic effect of thalidomide on inflammatory pain. Eur J Pharmacol 2000;391:97-103.

40. Sacerdote $P$, et al. Transient early expression of TNF-alpha in sciatic nerve and dorsal root ganglia in a mouse model of painful peripheral neuropathy. Neurosci Lett 2008;436:210-3.

41. Sakuma $Y$, et al. Up-regulation of p55 TNF alpha-receptor in dorsal root ganglia neurons following lumbar facet joint injury in rats. Eur Spine J 2007;16:1273-8.

42. Sampaio EP, et al. Thalidomide selectively inhibits tumor necrosis factor alpha production by stimulated human monocytes. J Exp Med 1991;173:699-703.

43. Schäfers $M$, et al. Anterograde transport of tumor necrosis factor-alpha in the intact and injured rat sciatic nerve. J Neurosci 2002;22:536-45.

44. Schäfers $M$, et al. Selective stimulation of either tumor necrosis factor receptor differentially induces pain behavior in vivo and ectopic activity in sensory neurons in vitro. Neuroscience 2008;157:414-23.

45. Schäfers M, et al. Intramuscular injection of tumor necrosis factor-alpha induces muscle hyperalgesia in rats. Pain 2003;104:579-88. 
46. Schafers $M$, et al. Tumor necrosis factor-alpha induces mechanical allodynia after spinal nerve ligation by activation of p38 MAPK in primary sensory neurons. J Neurosci 2003;23:2517-21.

47. Scholz J, et al. Blocking caspase activity prevents transsynaptic neuronal apoptosis and the loss of inhibition in lamina II of the dorsal horn after peripheral nerve injury. J Neurosci 2005;25:7317-23.

48. Sommer $\mathrm{C}$, et al. The effect of thalidomide treatment on vascular pathology and hyperalgesia caused by chronic constriction injury of rat nerve. Pain 1998;74:83-91.

49. Üçeyler $\mathrm{N}$, et al. Differential expression of cytokines in painful and painless neuropathies. Neurology 2007;69:42-9.

50. Vandenabeele $P$, et al. Two tumour necrosis factor receptors: structure and function. Trends Cell Biol 1995;5:392-9.

51. Xu JT, et al. The role of tumor necrosis factor-alpha in the neuropathic pain induced by Lumbar 5 ventral root transection in rat. Pain 2006;123:306-21.

52. Youn $\mathrm{DH}$, et al. Exogenous tumor necrosis factor-alpha rapidly alters synaptic and sensory transmission in the adult rat spinal cord dorsal horn. J Neurosci Res 2008;86:2867-75.

53. Zhang L, et al. TNF-alpha contributes to spinal cord synaptic plasticity and inflammatory pain: Distinct role of TNF receptor subtypes 1 and 2 . Pain 2011;152:419-27.

54. Zhang R, et al. Effects of TGF-beta1 and IGF-1 on proliferation of human nucleus pulposus cells in medium with different serum concentrations. J Orthop Surg Res 2006;1:9.

55. Zimmermann M. Ethical guidelines for investigations of experimental pain in conscious animals. Pain 1983;16:109-10. 


\section{Chapter}

TNF- $\alpha$ inhibitors alleviate experimentallyinduced neuropathic pain by downregulation of TNF receptors

Pablo Andrade, Veerle Visser-Vandewalle, John S. Del Rosario, Harry W.M. Steinbusch, Marc A. Daemen, Govert Hoogland 


\section{Chapter 8}

\section{Abstract}

Lesion of the sciatic nerve has been shown to induce a robust inflammatory response that is responsible for pain behavior. Among the agents involved in these events, TNF- $\alpha$ and its receptors (TNFRs) are considered as crucial participants in the development of pain. The present study examined TNFRs expression in the dorsal root ganglion (DRG) and dorsal horn (DH) after antiTNF- $\alpha$ administration in a rat chronic constriction injury $(\mathrm{CCl})$ model of painful peripheral neuropathy. The experiment design was the following: (1) the administration of saline solution, etanercept or infliximab was performed in $\mathrm{CCl}$ and sham operated animals. (2) Behavioral tests were carried out preoperatively and during the four consecutive postoperative weeks to assess the effects of TNF-a inhibition on pain recovery. (3) DRG and DH samples were collected to measure TNFRs levels in order to evaluate the effects of anti-TNFa treatment on receptor expression. (4) A correlation analysis between TNFRs expression and pain recovery was built to examine the impact of inflammation on behavior. The behavioral analysis showed that etanercept and infliximab treated animals displayed a greater and more accelerated recovery than controls. The latter was more efficient because it showed significant improvement on earlier stages and it was more consistent at the long-term testing. mRNA and protein analyses showed increased TNFRs levels in ipsilateral samples of $\mathrm{CCl}$ rats, but not in sham. Both treatments reduced significantly these levels, although infliximab showed a stronger effect to diminish the enhanced TNFRs expression. Meanwhile, the correlation between TNFRs expression and pain recovery evidenced that a deficient behavioral recovery is associated with an increased ratio of TNFR1 over TNFR2. Our results demonstrate that the attenuation of increased TNFRs levels after nerve injury is necessary to promote an earlier and successful recovery, and that infliximab may contribute to this effect by blocking TNFR1 action. 
TNF-alpha inhibitors reduce neuropathic pain

\section{Introduction}

It has been demonstrated that peripheral nerve injury and inflammation induce changes in the central nervous system that are responsible for central sensitization (Woolf, 1983; Woolf and Thompson, 1991; DeLeo and Yezierski, 2001; Ji et al., 2003;). This inadequate adaptation of the somatosensory nervous system can result in the reorganization of nociceptive processing; therefore, in the development of hypersensitivity in response to non-noxious and noxious stimuli (for review see Latremoliere and Woolf, 2009). Clinically, a wide variety of disorders that involve nerve damage and chronic pain have been shown to be associated with an inflammatory process (Üçeyler et al., 2007; Genevay et al., 2008; Alexander et al., 2012). In line with these observations, animal studies have demonstrated that peripheral inflammation after nerve lesion is a crucial participant for the initiation and maintenance of pain (Schäfers et al., 2003a, 2003b; Youn et al., 2008). In addition, it has been reported in injury-induced models that proinflammatory cytokine expression in the spinal cord correlate with pain behavior (DeLeo et al., 2000; Hatashita et al., 2008).

Tumor necrosis factor- $\alpha$ (TNF- $\alpha$ ) has been extensively implicated in many animal and human studies as an important proinflammatory agent that modulates pain (Covey et al, 2002; Empl et al., 2001). Following peripheral nerve injury, upregulation of TNF- $\alpha$ expression has been documented in several neuropathic pain-induced models. An increase expression of both forms of this cytokine, the membrane-bound (mTNF- $\alpha$ ) and the soluble trimeric forms (sTNF$\alpha$ ), have been described after nerve lesions in rats (Hao et al., 2007; George et al., 1999). The effects of TNF- $\alpha$ in these studies have been mainly associated with apoptotic modulation that eventually leads to cell death. Recently, several studies suggest that the definitive effects of TNF- $\alpha$ on inflammation and consequently on pain might be regulated by its receptors (TNFRs) (George et al., 2005; Sakuma et al., 2007; Constantin et al., 2008). This may relate to the observation that the two types of TNFRs, TNFR1 and TNFR2, follow different intracellular pathways and consequently induce distinct outcomes to the cell (for review see Andrade et al., 2011a). Likewise, TNFR1 and TNFR2 play different roles in the regulation of neuropathic and inflammatory pain (Schäfers et al., 2008; Zhang et al., 2011). Activation of TNFR1 results in apoptosis and has been associated with increased pain states (Dubový et al., 2006; Lee et al., 2009), whereas activated TNFR2 induces cell survival and proliferation, and it is linked to early-phase pain or diminished pain behavior (Zhang et al., 2011; Andrade et al., 2012). However, it remains unclear whether the differential modulation of pain behavior from the TNFRs is equally affected by activation 
with either the membrane or the soluble forms of TNF- $\alpha$, and how these mechanisms are affected by TNF- $\alpha$ inhibition. The aims of the present study were; first, to compare pain behavior with TNFRs expression in the dorsal root ganglion (DRG) and the dorsal horn (DH) of the spinal cord following a peripheral sciatic injury. Secondly, to investigate if the analgesic effect elicited by peripheral administration of different TNF- $\alpha$ inhibitors is followed by spinal changes in TNFRs levels. Finally, to investigate if pain behavior is differentially affected after blockage of sTNF- $\alpha$ or mTNF- $\alpha$.

\section{Experimental procedures}

All the experiments were carried out in compliance with the guidelines of the Experimental Animal Facility and Ethics Committee of Maastricht University and followed the ethical guidelines established by the International Association of the Study of Pain (IASP) for animal research (Zimmermann, 1983). All the efforts were made to minimize the amount of animals included in this study and to maximally reduce their suffering during the experiments.

\subsection{Animals and experimental groups}

Adult male Lewis rats (Charles River Laboratories, France) with an initial weight of 200-250g were used in all experiments. Animals were housed individually in standard cages with free access to food and water in a humidity and temperature-controlled room. The animals were allowed to habituate for 1 week to the housing environment before the surgery. Rats were randomly assigned to undergo a sham $(n=18)$ or $\mathrm{CCl}$ procedure $(n=30)$, and to receive one of the following therapies: $0.9 \% \mathrm{NaCl}$ (saline control), etanercept or infliximab. All drugs were dissolved in sterile $0.9 \% \mathrm{NaCl}$ to $0.5 \mathrm{ml}$ final volume and injected subcutaneously. Each animal received the same drug at two different occasions, i.e., immediately after the surgical procedure finished (in the contralateral limb) and on day 5 after the surgery (in the neck). Each injection contained (per kg bodyweight): $3 \mathrm{mg}$ etanercept (Wyeth, Münster, Germany) (Zanella et al., 2008) or $10 \mathrm{mg}$ infliximab (Centocor, Horsham PA, USA) (Murata et al., 2005). A schematic representation of the experimental timeline is shown in fig. 1. 


\subsection{Surgical procedures}

A detailed description of the surgical procedure has been reported previously (Andrade et al., 2012). In brief, the left sciatic nerve was exposed at the level of the mid-thigh under xylazine $(10 \mathrm{mg} / \mathrm{kg}$, s.c.) and ketamine $(90 \mathrm{mg} / \mathrm{kg}$, i.p.) anesthesia. After this, in sham cases the nerve did not receive additional manipulation. For the $\mathrm{CCl}$ cases, four loose ligatures (3-0 chromic gut) were tied around the nerve leaving $1 \mathrm{~mm}$ of distance between ligatures and allowing a normal blood supply (Bennett and Xie, 1988).

\subsection{Behavioral tests}

The withdrawal thresholds to tactile stimulation and the withdrawal latencies to heat were assessed one day before surgery and up to 4 weeks after the procedure at regular time points (postoperative weeks 1, 2, 3 and 4). An observer that was blinded to the surgical procedure and treatment group performed all tests. The mechanical hypersensitivity was assessed using the von Frey filaments test (Chaplan et al., 1994). Animals were tested on both hind paws using monofilaments filaments with gradual increasing stiffness starting from $1.4 \mathrm{~g}$ up to $100 \mathrm{~g}$ (Stoelting Co, Wood Dale, IL). The animals were allowed to acclimatize for 30 minutes to the plexiglass cages and the wire mesh before tactile stimulation in the mid-plantar surface. Each filament was tested 5 times before the next one was applied or until the withdrawal threshold was reached. This response was defined as the thinnest filament used to obtain a paw withdrawal in three out of five trials. The thermal hypersensitivity was assessed using the Hargreaves test (Hargreaves et al., 1988). The animals were allowed to acclimatize for 10 minutes to the plexiglass cages and the glass surface before infrared heat stimulation in the mid-plantar surface. The elapsed time to withdraw the limb from the heating source was defined as the withdrawal latency. To avoid tissue damage the time limit for the heating source was 20 seconds. Also, each maneuver was applied only once in each hind paw in one day.

\section{4. Tissue preparation for Western blot and qRT-PCR}

Half of the animals from each group were sacrificed at postoperative week 2 and the other half at postoperative week 4 . After the behavioral test, the 
animals were sedated using a pentobarbital injection (150 mg/kg, i.p.) followed by an ice-cold transcardial perfusion with saline solution. After flushing the spinal canal with ice-cold saline solution to obtain the spinal cord, the ipsi- and contralateral DRG and the ipsi- and contralateral DH of the spinal cord at the L4-L6 level were dissected. After tissue collection, samples were quickly frozen with liquid nitrogen and then stored at $-80^{\circ} \mathrm{C}$ before QRT-PCR or Western blot analysis.

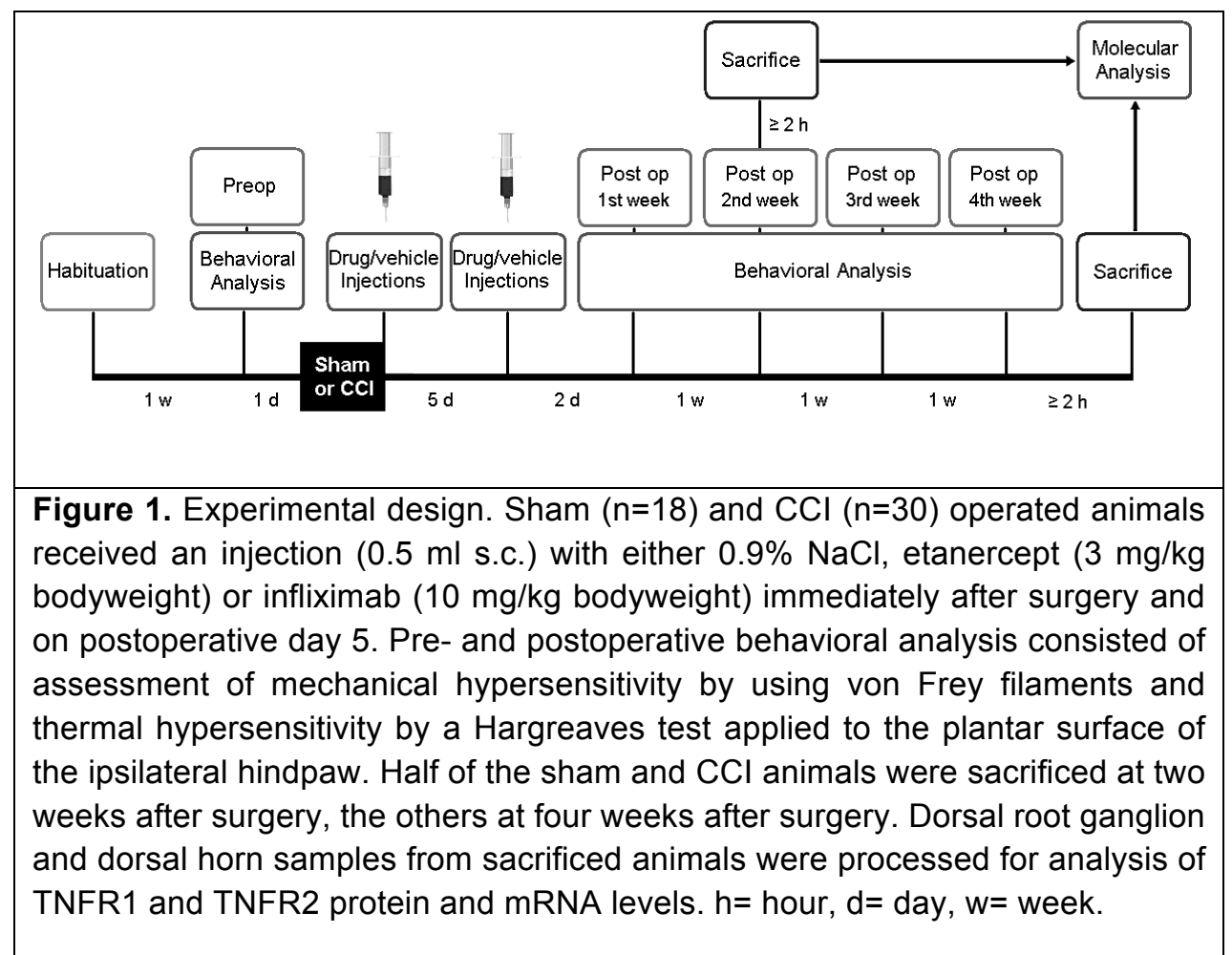

\section{5.Western blot}

The tissue was homogenized in ice-cold lysis buffer (phosphate buffered saline (PBS) containing 1\% Igepal, 0.1\% Triton X-100, 1 mM EDTA, 1 mM EGTA, and protease inhibitor cocktail (Roche Diagnostics, IN)). The same amount of protein $(20 \mu \mathrm{g})$ was loaded in each lane, separated by SDS- $10 \%$ polyacrylamide gel electrophoresis for $90 \mathrm{~min}$ at $100 \mathrm{~V}$ and then transferred to a pure 
nitrocellulose membrane (Bio-Rad Laboratories, CA) for $1 \mathrm{~h}$ at $100 \mathrm{~V}$. The membranes were rinsed with PBS and blocked in Odyssey blocking buffer (LICOR Biosciences, NE) 1:2 diluted in PBS for $1 \mathrm{~h}$, and were incubated overnight at $4^{\circ} \mathrm{C}$ with polyclonal rabbit-anti rat TNF-R1 $(1: 500 ; \mathrm{H}-271$, sc-7895, Santa Cruz Biotechnology) or polyclonal rabbit-anti rat TNF-R2 (1:500; H-202, sc7862, Santa Cruz Biotechnology). Monoclonal mouse-anti rat $\beta$-Actin (1:500; ACTBD11B7, sc-81178, Santa Cruz Biotechnology) was used as a loading control for both receptors. Next, membranes were rinsed with PBS and incubated for $1 \mathrm{~h}$ with 1:10.000 Alexa Fluor 800 goat anti-rabbit IgG (Molecular Probes, OR) for the receptors and 1:10.000 Alexa Fluor 680 donkey anti-mouse IgG (Molecular Probes, OR) for $\beta$-Actin. Subsequently, membranes were rinsed with PBS, and finally dried and visualized by enhanced chemiluminescence detection with an Odyssey Infrared Imaging System (LI-COR Biosciences). The relative density of immunoreactive bands was quantified by calculating the optical density ratio of the band of interest over that of $\beta$-Actin using ImageJ $1.42 q$ software (National Institute of Health, MD).

\section{6. $q R T-P C R$}

The total RNA was extracted using the reagent TRIzol (Invitrogen, CA) according to the manufacturer protocol. $1 \mu \mathrm{g}$ total RNA was reversely transcribed to cDNA using a First Strand cDNA Synthesis Kit (Fermentas International Inc., Ontario, Canada). The relative mRNA expression of TNFR1 and TNFR2 was quantified by qRT-PCR using a LightCycler 480 Real-Time PCR System (Roche Diagnostics). The following primer sets were used for the TNFRs: TNFR1-forward 5'- CTC TTG GTG ACC GGG AGA AG -3' and reverse 5'- GGT TCC TTT GTG GCA CTT GGT -3', and TNFR2-forward 5'- CAT CCC TGT GTC CTT GGG $-3^{\prime}$ and reverse $5^{\prime}$ - CCC GTG ATG CTT GGT TCA - 3 '. Amplicons for the reference gene glyceraldehyde 3-phosphate dehydrogenase (GAPDH) were generated using the following primer sets: forward primer 5'GCA AGA GAG AGG CCC TCA G $-3^{\prime}$ and reverse 5'- TGT GAG GGA GAT GCT CAG TG - $3^{\prime}$. The initial denaturation step was set at $94^{\circ} \mathrm{C}$ for $30 \mathrm{~s}$, and it was followed for 50 cycles by reaction steps of $63^{\circ} \mathrm{C}$ for $15 \mathrm{~s}$ and $72^{\circ} \mathrm{C}$ for $15 \mathrm{~s}$. In order to verify product specificity, we performed melting curve analysis and $2 \%$ agarose gel on the reaction samples. Data were analyzed by the delta CT method and normalized to the reference gene. 


\section{7. Statistical analysis}

All data in this study were expressed as mean \pm SEM. For statistical analysis GraphPad Prism 5 software was used. Since the behavioral and molecular data did not show a normal distribution, a Kruskal-Wallis test was used in $\mathrm{CCl}$ animals to analyze differences between drug treatments. A Mann-Whitney $U$ test was used for comparison between two groups. A Bonferroni correction was used to investigate differences with the $\mathrm{NaCl}$ injected group. $\mathrm{P}$ values $<0.05$ were considered significant.

\section{Results}

\section{1. TNF- $\alpha$ inhibition reduced mechanical and thermal hypersensitivity on $\mathrm{CCl}$ rats}

Behavioral testing showed that before the surgical procedure animals withdrew ipsilateral and contralateral hind limbs similarly from mechanical stimulation when von Frey filaments of about $100 \mathrm{~g}$ were used, and a withdrawal latency threshold of about $14.5 \mathrm{~s}$ when the laser-heating source was applied. Postsurgically, sham and $\mathrm{CCl}$ operated animals showed similar behavior on the contralateral hind paw for the following four weeks (fig. 2 A, B). During the four postsurgical weeks, mechanical allodynia was observed in the ipsilateral hind paw of $\mathrm{CCl}$ operated animals, but not in sham animals (fig. 2A). However, etanercept and infliximab treated rats showed significantly higher withdrawal thresholds to mechanical stimuli than saline treated rats during the four weeks of postsurgical evaluation. At one week after surgery, the infliximab treated rats was the only group to show a significantly higher threshold when compared to saline controls $(p<0.05)$. At 2 and 3 weeks after $\mathrm{CCl}$ surgery this threshold had further increased $(p<0.01)$ and also etanercept treated animals showed significantly higher withdrawal thresholds $(p<0.05)$ to mechanical stimuli when compared to saline controls. Finally, at 4 weeks after surgery, infliximab treated animals still showed a significantly higher withdrawal threshold $(p<0.05)$, but not etanercept treated animals. This may be linked to a mild increase on the withdrawal threshold showed by saline controls. 
A

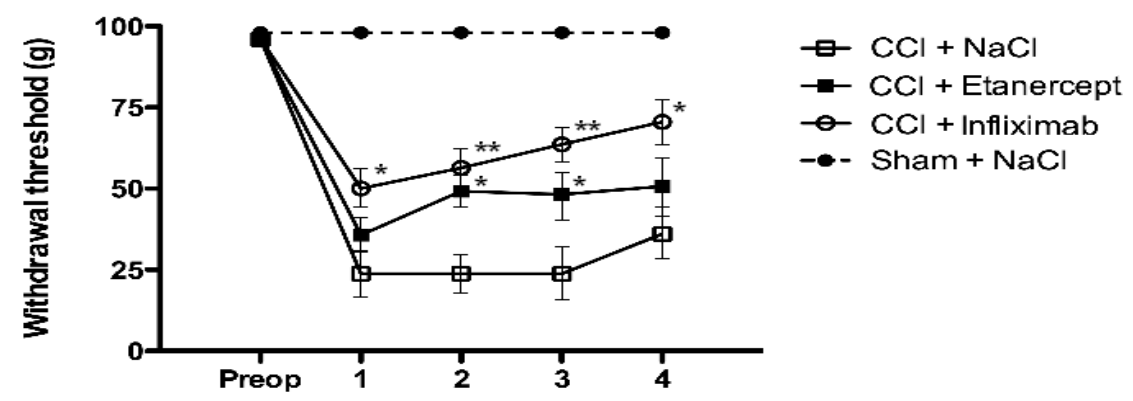

Postoperative time (weeks)

B

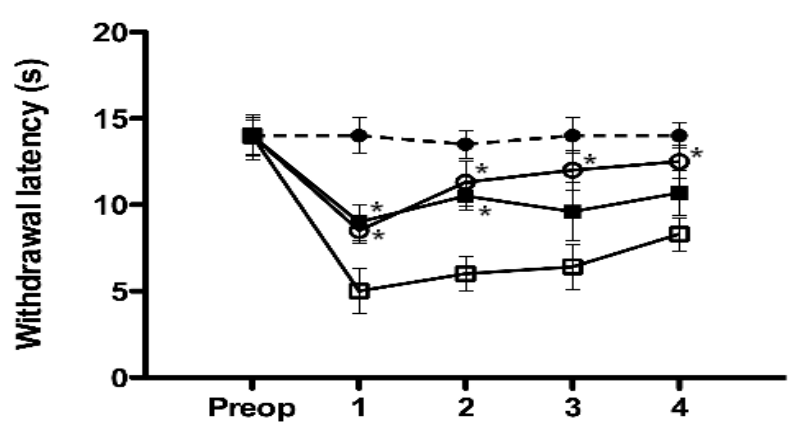

Postoperative time (weeks)

Figure 2. Etanercept and infliximab facilitate recovery of mechanical $(A)$ and thermal (B) hypersensitivity after $\mathrm{CCl}$. Only infliximab was able to maintain a significant recovery in both tests throughout the 4 postoperative weeks. Data are expressed as mean \pm SEM of ipsilateral hind paw observations from 5 to 10 animals. ${ }^{*} p<0.05$ and ${ }^{* *} p<0.01$ compared to $\mathrm{CCl}+\mathrm{NaCl}$ injected rats. $g=$ grams, $\mathrm{s}=$ seconds.

Similar to mechanical stimulation, during the four postsurgical weeks, thermal hyperalgesia was observed in the ipsilateral hind paw of $\mathrm{CCl}$ operated animals, but not in sham animals (fig. 2B). As well, etanercept and infliximab treated animals showed a similar recovery after thermal stimulation from that one 
observed on the mechanical hypersensitivity evaluation. At one and two weeks after $\mathrm{CCl}$ surgery etanercept and infliximab treated rats showed significantly longer withdrawal latencies $(p<0.05)$ than saline treated rats. At three and four weeks after surgery this latency further increased only in the case of infliximab treated animals which still showed a significantly longer withdrawal latency $(p<0.05)$ when compared to saline treated rats. At the same period of three and four weeks after surgery, the withdrawal latencies of etanercept treated rats decreased and increased erratically. Even though these latencies showed an improvement tendency they were not significant when they were compared to saline controls. This may also be associated to a mild gradual recovery from $\mathrm{CCl}$-induced thermal hypersensitivity showed by the saline treated animals throughout the four postsurgical weeks.

\section{2. Increased expression of TNFR protein levels after $\mathrm{CCl}$ is reduced with etanercept and infliximab administration}

TNFR1 (55 kD) was observed in homogenized DRG and DH samples collected at 2 and 4 weeks after surgery from sham operated animals, but not TNFR2 (75 $\mathrm{kD}$ ). Similarly, in the case of $\mathrm{CCl}$ operated animals the contralateral DRG and DH samples collected at 2 and 4 weeks after surgery expressed only TNFR1. The level of TNFR1 expression in the $\mathrm{CCl}$ groups was not significantly different from the level in the sham group (data not shown in the figures). On the contrary, homogenized ipsilateral DRG and DH samples from $\mathrm{CCl}$ animals collected at 2 and 4 weeks after surgery expressed both, TNFR1 and TNFR2 (fig. 3). At 2 weeks after surgery, etanercept and infliximab treated rats expressed significantly reduced TNFR1 proteins levels in DRG and DH samples from $\mathrm{CCl}$ rats compared from those of saline controls (fig. 3A, B). At 4 weeks after surgery, the expression of TNFR1 protein levels in DRG and DH samples from $\mathrm{CCl}$ rats was significantly reduced only in the infliximab group. For TNFR2, the expression of protein levels was reduced by about $40 \%$ at 2 weeks after surgery in DRG and $\mathrm{DH}$ samples from $\mathrm{CCl}$ rats treated with infliximab (fig. $3 \mathrm{C}$, D). At 4 weeks after surgery, the expression of TNFR2 protein levels were significantly reduced in the in the $\mathrm{DH}$ samples of $\mathrm{CCl}$ rats treated with etanercept and infliximab, but not in the DRG samples. 


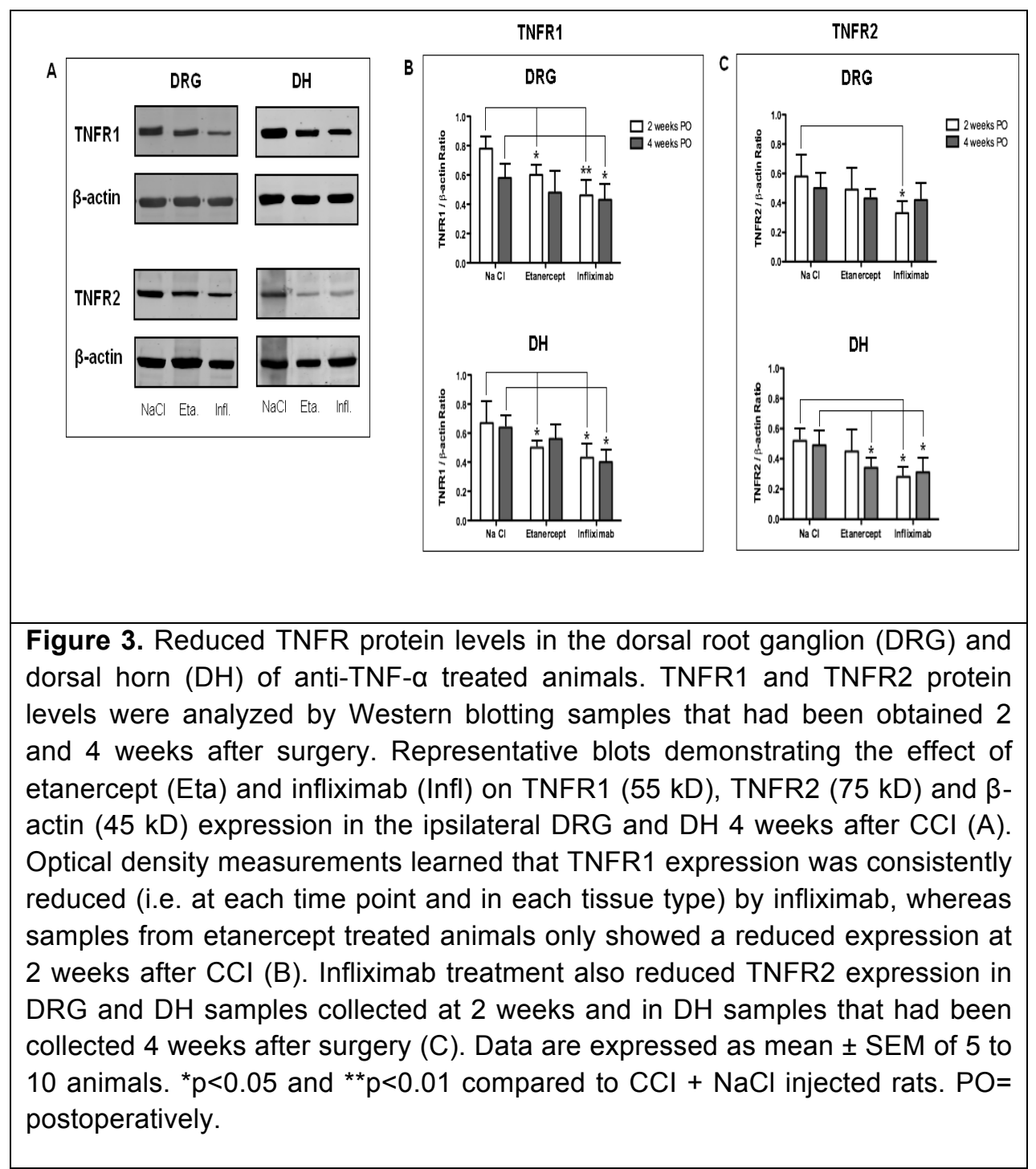

\section{3. Diminished mRNA TNFR levels in anti-TNF- $\alpha$ treated animals correlate with decreased pain behavior}

TNFR2 mRNA was undetectable in DRG and DH samples from sham operated animals. TNFR1 mRNA expression in DRG and DH samples from sham operated animals was low, and it was comparable to TNFR1 mRNA levels in contralateral DRG and $\mathrm{DH}$ samples from $\mathrm{CCl}$ animals (data not shown in 
figures). At 2 and 4 weeks after $\mathrm{CCl}$ surgery, TNFR1 and TNFR2 mRNA expression in DRG and DH samples from saline treated animals was similar between the two time points (fig. 4). In this group the ipsilateral samples expressed 6 to 7 times more TNFR1 mRNA copies than the contralateral DRG and DH samples (fig. 4A, B). Whereas for TNFR2 levels, ipsilateral samples expressed about 4 times more mRNA copies than the contralateral DRG and DH samples (fig. 4C, D).

Etanercept and infliximab treated animals expressed significantly lower TNFR1 mRNA levels in DRG and DH samples compared to saline controls. This effect was observed for etanercept at 2 weeks after surgery in DRG samples where (fig. 4A). While for infliximab the treatment effect was evident at 2 and 4 weeks after surgery in both DRG and DH samples (fig. 4A, B). Comparatively, TNFR2 mRNA expression at 2 and 4 weeks showed a significant treatment effect in infliximab treated rats in DRG and DH samples (fig. 4C, D). Whereas in etanercept treated rats there was a significantly reduced TNFR2 expression compared to saline controls at 4 weeks after surgery only in $\mathrm{DH}$ samples (fig. 4D).

To investigate if an imbalance between TNFR1 and TNFR2 was associated with TNF- $\alpha$ inhibitors administration, we compared the TNFR1 / TNFR2 mRNA ratio from saline controls with the ratio from etanercept and infliximab treated rats (fig. 5A). In addition, to examine if pain behavior was accompanied by altered TNFR expression, we compared the TNFR1 / TNFR2 mRNA ratio of poor recovery $(<50 \%$ recovery from the presurgical value of the von Frey filament withdrawal threshold or the heating-source withdrawal latency) with that of good recovery $(\geq 50 \%$ recovery from the presurgical value of the von Frey filament withdrawal threshold or the heating-source withdrawal latency) in $\mathrm{CCl}$ animals (fig. $5 \mathrm{~B}, \mathrm{C}$ ). The degree of recovery was calculated by establishing individually the presurgical value from each behavioral test as a score of $100 \%$. A significantly increased TNFR1 / TNFR2 mRNA ratio was observed in animals that exhibited a poor recovery. This analysis showed that rats with a poor recovery from mechanical hypersensitivity have significantly higher TNFR1 levels over TNFR2 in DH samples (fig. 5B). Whereas animals with a poor recovery from thermal hypersensitivity have significantly higher TNFR1 levels over TNFR2 in DRG and DH samples (fig. 5C). 


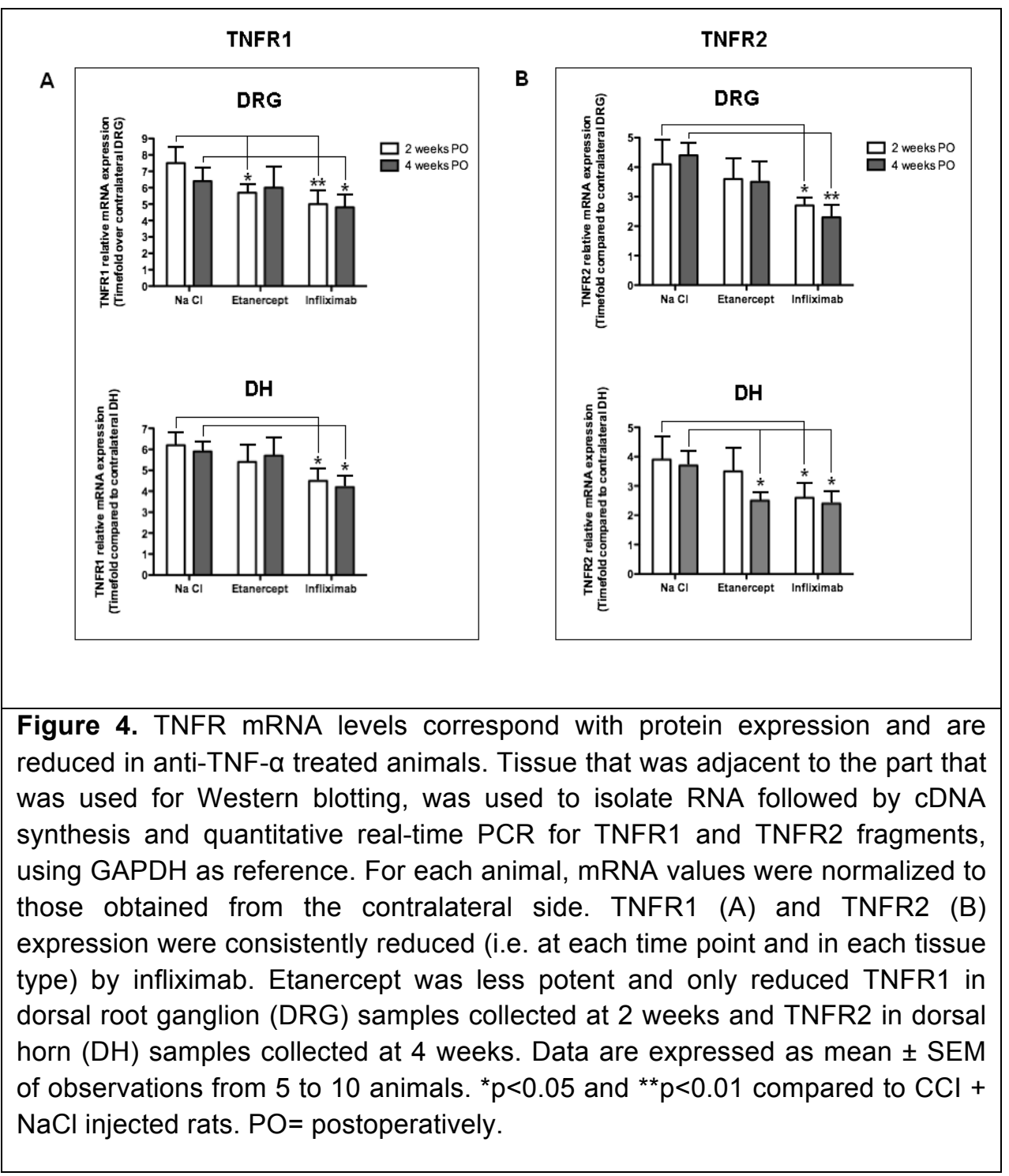

\section{Discussion}

The present data indicate that etanercept and infliximab can indirectly inhibit TNFR expression after blocking TNF- $\alpha$ production secondary to a nerve lesion. These treatments can significantly reduce the mRNA and protein expression of TNFR1 and TNFR2 in peripheral (DRG) and central (DH) nervous system after 


\section{Chapter 8}

$\mathrm{CCl}$ of the sciatic nerve. TNF- $\alpha$ inhibition induced substantial changes in the expression pattern of TNFR by suppressing the anterograde transport of TNF- $\alpha$ from the periphery to the spinal cord. In addition, the reduction on TNFR levels was correlated with higher withdrawal thresholds and an accelerated recovery after periphery nerve injury. Moreover, the severity of pain behavior in animals with a poor outcome was associated with an increased TNF R1 / R2 ratio. These findings suggest that the decrease of $\mathrm{CCl}$-induced mechanical and thermal hypersensitivity generated by etanercept and infliximab is regulated by changes in TNFR expression on the periphery and the spinal cord.

An increased TNFR expression in the nervous system has been associated with enhanced pain states in animal and human studies (Dubový et al., 2006; Zhang et al., 2011; Andrade et al., 2011b). These findings suggest a direct correlation between high levels of TNFR and an augmentation in the severity of pain. In our study, we found that $\mathrm{CCl}$-induced inflammation was accompanied by increased pain behavior and TNFR expression in saline controls. This enhanced hypersensitivity to mechanical and thermal stimuli secondary to peripheral nerve lesion was reduced when the animals where injected with etanercept or infliximab. Furthermore, the levels of TNFR expression in the $D R G$ and DH after nerve lesion were significantly lower in etanercept and infliximab treated animals. It is important to remark that the enhanced mechanical and thermal hypersensitivity and the increased TNFR expression of $\mathrm{CCl}$ rats remained ipsilateral to the nerve lesion. These findings were not present in the contralateral hindpaw or the contralateral DRG and DH samples, respectively. These results are in line with previous data from our group, where we showed that the blockage of TNF- $\alpha$ production reduces TNFR expression in the spinal cord and ultimately promotes pain recovery (Andrade et al., 2012). Interestingly in the current study, the effects of etanercept and infliximab differed temporally and quantitatively on pain behavior, even when they share similar mechanisms of action. At every measurement, infliximab proved to be a more effective treatment than etanercept against inflammatory induced chronic pain. The former was the only drug that significantly increased the withdrawal thresholds and latencies from the early to the late phase of the inflammatory process, and the latter was inconsistent on pain recovery especially at the late phase. In the same way, for mRNA and protein expression, infliximab was the only constant treatment that maintained significantly lower levels of TNFR expression at 2 and 4 weeks after surgery. These differences could be explained because of their different capacity to bind the two circulating forms of TNF- $\alpha$, sTNF- $\alpha$ and mTNF- $\alpha$. It is known that etanercept has a very high affinity for sTNF- $\alpha$, especially for the trimeric form (Bhatia and Kast, 2007). Whereas 
infliximab has a higher affinity for mTNF- $\alpha$ than for sTNF- $\alpha$ (Scallon et al., 2002), although its binding to STNF- $\alpha$ is more stable than the complexes created by etanercept (Mpofu et al., 2005). This may be relevant for TNFR expression and pain behavior since sTNF- $\alpha$ binds in its majority to TNFR1 (Bhatia and Kast, 2007), while mTNF- $\alpha$ exerts its effects preponderantly through TNFR2 activation (Faustman and Davis, 2010). Some lines of evidence even suggest the possibility that TNFR2 activation can only be achieved through mTNF-a binding (Grell et al., 1995; Krippner-Heidenreich A et al., 2002; Faustman and Davis, 2010).

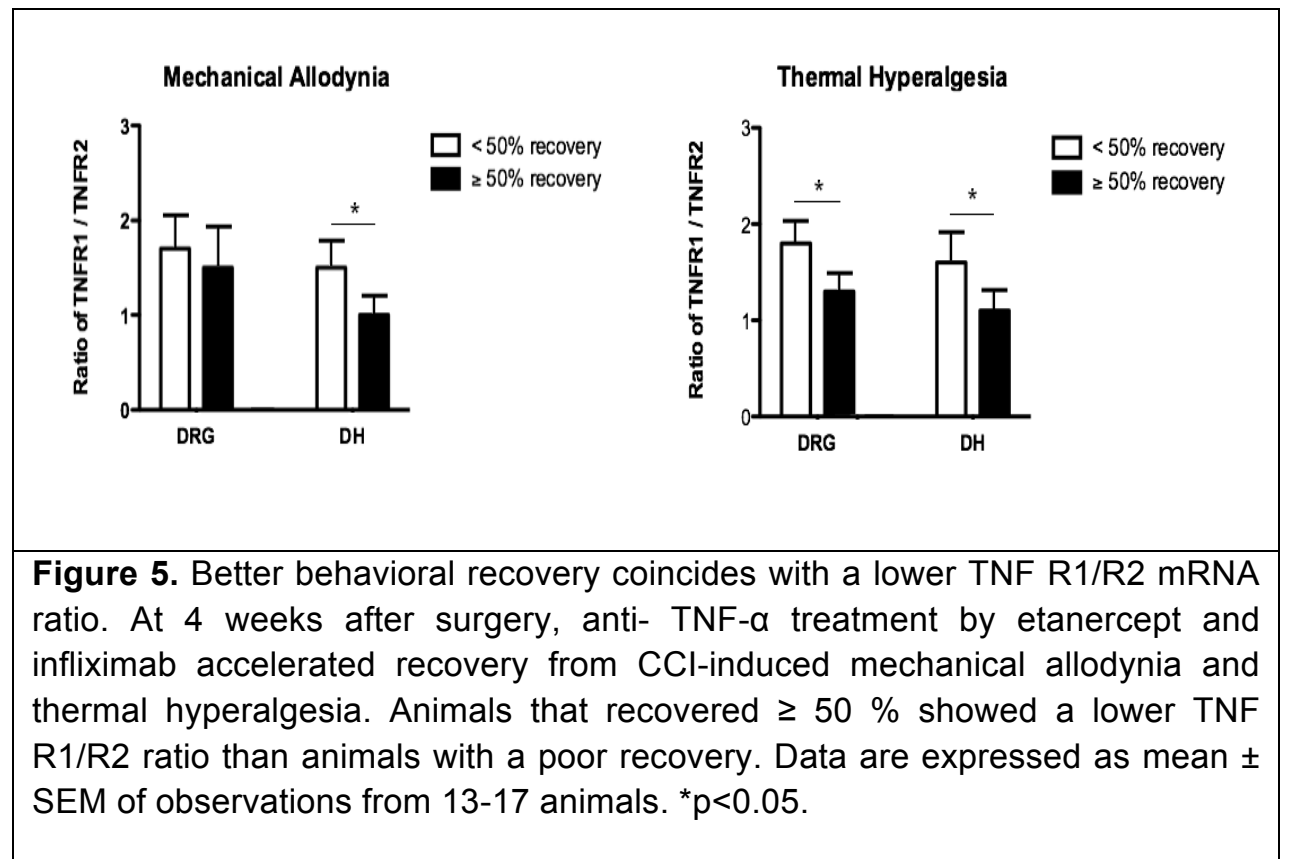

Previous preclinical studies have demonstrated the analgesic effects of antiTNF- $\alpha$ drugs in animal models of inflammatory pain (Murata et al., 2005; Zanella et al., 2008). However, their efficacy in human trials has not been reproduced convincingly for many chronic pain states (Autio et al., 2006; Korhonen et al., 2005; Cohen et al., 2007). Moreover, in some rheumatic disorders their use has been associated with the worsening of symptoms, suggesting that side effects may result from positive reinforcement for TNF- $\alpha$ synthesis (Bhatia and Kast, 2007). Notwithstanding these observations, the analgesic effects of TNF- $\alpha$ inhibitors like etanercept, infliximab and adalimumab 


\section{Chapter 8}

are largely considered positive and are still promising for further indications in pain disorders (Genevay et al, 2012; Mpofu et al., 2005). It is feasible that one of the explanations for this restricted effect is associated with the selective inhibition performed by these drugs. As we previously mentioned, these inhibitors possess different affinities and binding stabilities to the distinct TNF- $\alpha$ forms that could affect the blockage of the cytokine. Thus, an incomplete inhibition could take place if one of these treatments is used. In addition, the suggested mechanism of these drugs is derived from their capacity to bind and competitively block circulating TNF- $\alpha$. This means that the synthesis and release of TNF- $\alpha$ is not antagonized, and therefore it may have the opportunity to exert its effects over other agents and cytokines dependent of its release, i.e. IL-6 (Charles et al., 1999). In this respect, drugs like thalidomide may offer an interesting complementary effect in order to suppress the production of TNF- $\alpha$ from the cells (Peterson et al., 1995; Sommer et al., 1998). It may be possible that a combination of these anti-inflammatory treatments may provide better results with fewer side effects. By doing this, we could prevent the release of TNF- $\alpha$ in early phases and inhibit its effects at the late stage, i.e. if we use thalidomide right after a nerve lesion to prevent the synthesis and etanercept or infliximab weeks after the damage to inhibit the circulating TNF- $\alpha$. Furthermore, in case that both therapies are administered at the same time, it could be possible to reduce their doses in order to avoid side effects. In the future, these insights may help us to design new anti-inflammatory therapy schemes with drugs that are currently available.

The anterograde transport of TNF- $\alpha$ from the periphery into the spinal cord has been shown in other studies of $\mathrm{CCl}$ (Shubayev and Myers, 2001; Schäfers et al., 2002). Here, we show that the TNFR expression pattern after peripheral nerve lesion does not follow the same tendency as the cytokine. Interestingly, the receptor expression did not show a clear delay between DRG and DH samples from the same animal at the same time point. In other words, the receptor expression was similar between two different anatomical sites at the same time, in samples from the same animal. These findings suggest that at the moment when the insult is detected and recognized by its receptors in the periphery, the activation of further TNFR occur in the spinal cord in a short period of time. However, when the molecular data is matched with the behavior, the most determining factor to affect the outcome was the increased ratio of TNFR1 over TNFR2. This last observation corroborate previous data from our group where we show that high TNFR1 levels are associated with increased hypersensitivity, whereas high TNFR2 levels are present in animals with a 
better recovery (Andrade et al., 2012). All these findings taken together may have further implications for pain treatment by specific targeting of TNFR.

\section{Conclusion}

Data from the present study indicate that etanercept and infliximab diminish pain behavior by reducing the expression of TNFR in the peripheral and central nervous system, after TNF- $\alpha$ inhibition. Infliximab was the most successful treatment to treat pain, which may be secondary to its higher capacity to block mTNF- $\alpha$ action. TNFR expression after peripheral nerve injury induces simultaneous expression of its receptors in the spinal cord. The most determining factor responsible for pain secondary to $\mathrm{CCl}$ of the sciatic nerve was an unbalanced increase of TNFR1 levels over TNFR2.

\section{References}

1. Alexander GM, et al. Changes in plasma cytokines and their soluble receptors in complex regional pain syndrome. J Pain 2012;13:10-20.

2. Andrade $P$, et al. The thalidomide analgesic effect is associated with differential TNF- $\alpha$ receptor expression in the dorsal horn of the spinal cord as studied in a rat model of neuropathic pain. Brain Res 2012;1450:24-32.

3. Andrade $P$, et al. Role of TNF-alpha during central sensitization in preclinical studies. Neurol Sci 2011a;32:757-771.

4. Andrade $P$, et al. Tumor necrosis factor-alpha levels correlate with postoperative pain severity in lumbar disc hernia patients: Opposite clinical effects between tumor necrosis factor receptor 1 and 2. Pain 2011b;152:2645-2652.

5. Autio RA, et al. The effect of infliximab, a monoclonal antibody against TNF-alpha, on disc herniation resorption: a randomized controlled study. Spine 2006;31:2641-5.

6. Bennett GJ, Xie YK. A peripheral mononeuropathy in rat that produces disorders of pain sensation like those seen in man. Pain 1988;33:87107.

7. Bhatia A, Kast RE. Tumor necrosis factor (TNF) can paradoxically increase on etanercept treatment, occasionally contributing to TNFmediated disease. J Rheumatol 2007;34:447-9. 
8. Chaplan SR, et al. Quantitative assessment of tactile allodynia in the rat paw. J Neurosci Methods 1994;53:55-63.

9. Charles $P$, et al. Regulation of cytokines, cytokine inhibitors, and acutephase proteins following anti-TNF-alpha therapy in rheumatoid arthritis. J Immunol 1999;163:1521-8.

10. Cohen SP, et al. A double-blind, placebo-controlled, dose-response pilot study evaluating intradiscal etanercept in patients with chronic discogenic low back pain or lumbosacral radiculopathy. Anesthesiology 2007;107:99-105.

11. Constantin CE, et al. Endogenous tumor necrosis factor alpha (TNFalpha) requires TNF receptor type 2 to generate heat hyperalgesia in a mouse cancer model. J Neurosci 2008;28:5072-5081.

12. Covey WC, et al. Expression of neuron-associated tumor necrosis factor alpha in the brain is increased during persistent pain. Reg Anesth Pain Med 2002;27:357-66.

13. DeLeo JA, et al. Transgenic expression of TNF by astrocytes increases mechanical allodynia in a mouse neuropathy model. Neuroreport 2000;11:599-602.

14. DeLeo JA, Yezierski RP. The role of neuroinflammation and neuroimmune activation in persistent pain. Pain 2001;90:1-6.

15. Dubový $P$, et al. Intra- and extraneuronal changes of immunofluorescence staining for TNF-alpha and TNFR1 in the dorsal root ganglia of rat peripheral neuropathic pain models. Cell Mol Neurobiol 2006;26:1205-1217.

16. Empl M, et al. TNF-alpha expression in painful and nonpainful neuropathies. Neurology 2001;56:1371-1377.

17. Faustman D, Davis M. TNF receptor 2 pathway: drug target for autoimmune diseases. Nat Rev Drug Discov 2010;9:482-93.

18. Genevay S, et al. Elevated levels of tumor necrosis factor-alpha in periradicular fat tissue in patients with radiculopathy from herniated disc. Spine 2008;33:2041-2046.

19. Genevay $S$, et al. Adalimumab in acute sciatica reduces the long-term need for surgery: a 3-year follow-up of a randomised double-blind placebo-controlled trial. Ann Rheum Dis 2012;71:560-2.

20. George A, et al. Tumor necrosis factor receptor 1 and 2 proteins are differentially regulated during Wallerian degeneration of mouse sciatic nerve. Exp Neurol 2005;192:163-166. 
21. George A, et al. Serial determination of tumor necrosis factor-alpha content in rat sciatic nerve after chronic constriction injury. Exp Neurol 1999;160:124-132.

22. Grell $M$, et al. The transmembrane form of tumor necrosis factor is the prime activating ligand of the $80 \mathrm{kDa}$ tumor necrosis factor receptor. Cell 1995;83:793-802.

23. Hao $S$, et al. Gene transfer to interfere with TNFalpha signaling in neuropathic pain. Gene Ther 2007;14:1010-1016.

24. Hargreaves $\mathrm{K}$, et al. A new and sensitive method for measuring thermal nociception in cutaneous hyperalgesia. Pain 1988;32:77-88.

25. Hatashita $S$, et al. Contralateral neuropathic pain and neuropathology in dorsal root ganglion and spinal cord following hemilateral nerve injury in rats. Spine 2008;33:1344-1351.

26. Ji RR, et al. Central sensitization and LTP: Do pain and memory share similar mechanisms? Trends Neurosci 2003;26:696-705.

27. Korhonen $\mathrm{T}$, et al. The treatment of disc herniation-induced sciatica with infliximab: results of a randomized, controlled, 3-month follow-up study. Spine 2005;30:2724-8.

28. Krippner-Heidenreich $A$, et al. Control of receptor-induced signaling complex formation by the kinetics of ligand/receptor interaction. J Biol Chem 2002;277:44155-63.

29. Latremoliere A, Woolf CJ. Central sensitization: a generator of pain hypersensitivity by central neural plasticity. J Pain 2009;10:895-926.

30. Lee KM, et al. Tumor necrosis factor receptor 1 induces interleukin-6 upregulation through NF-kappaB in a rat neuropathic pain model. Eur $\mathrm{J}$ Pain 2009;13:794-806.

31. Mpofu S, et al. Anti-TNF-alpha therapies: they are all the same (aren't they?). Rheumatology (Oxford) 2005;44:271-3.

32. Murata $Y$, et al. Effects of selective tumor necrosis factor-alpha inhibition to pain-behavioral changes caused by nucleus pulposusinduced damage to the spinal nerve in rats. Neurosci Lett 2005;382:148-152.

33. Peterson PK, et al.Thalidomide Inhibits Tumor Necrosis Factor- $\alpha$ Production by Lipopolysaccharide-and Lipoarabinomannan-Stimulated Human Microglial Cells. J Infect Dis 1995;172:1137-1140.

34. Scallon B, et al. Binding and functional comparisons of two types of tumor necrosis factor antagonists. J Pharmacol Exp Ther 2002;301:418-26. 
35. Schäfers M, et al. Anterograde transport of tumor necrosis factor-alpha in the intact and injured rat sciatic nerve. J Neurosci 2002;22:536-45.

36. Schäfers $M$, et al. Selective stimulation of either tumor necrosis factor receptor differentially induces pain behavior in vivo and ectopic activity in sensory neurons in vitro. Neuroscience 2008;157:414-423.

37. Schäfers $M$, et al. Intramuscular injection of tumor necrosis factor-alpha induces muscle hyperalgesia in rats. Pain 2003a;104:579-588.

38. Schäfers $M$, et al. Tumor necrosis factor-alpha induces mechanical allodynia after spinal nerve ligation by activation of p38 MAPK in primary sensory neurons. J Neurosci 2003b;23:2517-2521.

39. Shubayev VI, Myers RR. Axonal transport of TNF-alpha in painful neuropathy: distribution of ligand tracer and TNF receptors. J Neuroimmunol 2001;114:48-56.

40. Sommer C, et al.The effect of thalidomide treatment on vascular pathology and hyperalgesia caused by chronic constriction injury of rat nerve. Pain 1998;74:83-91.

41. Üçeyler $\mathrm{N}$, et al. Differential expression of cytokines in painful and painless neuropathies. Neurology 2007;69:42-49.

42. Woolf CJ. Evidence for a central component of post-injury pain hypersensitivity. Nature 1983;306:686-688.

43. Woolf CJ, Thompson SW. The induction and maintenance of central sensitization is dependent on N-methyl-D-aspartic acid receptor activation; implications for the treatment of post-injury pain hypersensitivity states. Pain 1991;44:293-299.

44. Sakuma Y, et al. Up-regulation of p55 TNF alpha-receptor in dorsal root ganglia neurons following lumbar facet joint injury in rats. Eur Spine J 2007; $16: 1273-1278$.

45. Youn $\mathrm{DH}$, et al. Exogenous tumor necrosis factor-alpha rapidly alters synaptic and sensory transmission in the adult rat spinal cord dorsal horn. J Neurosci Res 2008;86:2867-2875.

46. Zanella JM, et al. Effect of etanercept, a tumor necrosis factor-alpha inhibitor, on neuropathic pain in the rat chronic constriction injury model. Spine 2008;33:227-234.

47. Zhang $L$, et al. TNF-alpha contributes to spinal cord synaptic plasticity and inflammatory pain: Distinct role of TNF receptor subtypes 1 and 2 . Pain 2011;152:419-427.

48.Zimmermann M. Ethical guidelines for investigations of experimental pain in conscious animals. Pain 1983;16:109-110. 


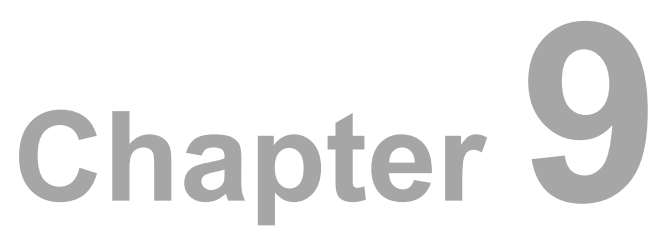

Summary and conclusion

Pablo Andrade 


\section{Chapter 9}

\section{Summary and conclusion}

In the present thesis, we have studied the correlation between TNF- $\alpha$ and its receptors and the severity of postoperative radicular leg pain in LDH patients after discectomy. In addition, we examined the opposite clinical effects between TNFR1 and TNFR2 in the modulation of postoperative pain, the former being related to elevated pain and the latter to a lower intensity or absence of pain. Following this, we have investigated if the expression of these markers was associated with an unsuccessful long-term outcome in terms of pain sensation and the necessity of performing multiple surgeries in LDH patients. Finally, animal experiments were performed to gain insights into the mechanisms of the analgesic effects produced by TNF- $\alpha$ inhibition. Furthermore, we have investigated if peripheral blockage of TNF- $\alpha$ could hinder the expression of TNFR in the central nervous system of rats and therefore the development of pain behavior. The summarized results reported in this thesis are presented as follows;

In chapter 2 of this thesis we have summarized the current available knowledge from lesional, transgenic and pharmacological preclinical models that evidences the role of TNF- $\alpha$ as a key modulator in pain. This is done from a behavioral standpoint, where the pain behavior is observed as a direct result from TNF- $\alpha$ stimulation. This literature analysis showed that early and late changes of TNF- $\alpha$ levels in nervous and non-nervous tissue have a temporal and simultaneous effect on pain behavior. In addition, in this overview we delineate one of our research questions in relation to the relevance of the TNFR1 / TNFR2 expression ratio as an important factor in the generation of pain.

In chapter 3, we provided an extensive overview of the correlation between inflammation and chronic pain in humans. Here, we summarized the current state of knowledge regarding cytokine expression in human samples in direct association with clinical pain assessment. From this revision we can conclude that inflammation is a constant factor present in a wide range of chronic disorders related to pain. There is enough scientific evidence to suggest that an unbalance favoring proinflammatory cytokines is related to pain. In contrast, a decreased anti-inflammatory cytokines expression may reduce pain.

In our initial human studies, as described in chapter 4 , we therefore analyzed TNF- $\alpha$ and TNFR levels in annulus fibrosus (AF), nucleus pulposus (NP), ligamentum flavum (LF) and paravertebral muscle biopsies that were collected 
in the course of a discectomy in LDH patients. The expression of these molecules in the NP correlate to the severity of postoperative radicular leg pain, indicating that these biomarkers may be employed to predict the clinical outcome of a discectomy. These observations furthermore suggest that in LDH patients with high TNF- $\alpha$ levels, anti-TNF- $\alpha$ therapy may be more effective to relief pain than a discectomy. In this respect it is interesting to note that increased TNFR1 levels correlated to increased pain, whereas TNFR2 was decreasedly expressed in patients with more pain. This differential TNFR expression may proof useful for future rationale drug design that is directed towards specific TNFR subtypes in order to reduce side effects.

Additional experiments described in chapter 5 show that the LDH biopsies also contain IL-1 $\beta$ and IL-6, indicating that these tissues are characterized by a general proinflammatory state. The cytokine levels, however, did not correlate with pre- or postoperative leg pain, suggesting that anti-TNF- $\alpha$ therapies may be more successful in pain management than anti-inflammatory ones. These observations reinforce the idea that once an inflammatory process is established, TNF- $\alpha$ may be one of the central players in the chronification of pain.

To further investigate their role in chronic pain, we analyzed TNF- $\alpha$ and TNFR expression in spinal biopsies from patients with recurrent LDH as described chapter 6. In line with our previous findings, patients that had undergone multiple spinal surgeries reported higher pain scores and expressed higher TNF- $\alpha$ and TNFR1 levels then patients that had undergone one LDH surgery. Together, these findings provide more evidence that local disc inflammation in $\mathrm{LDH}$ patients is closely involved in persistent postoperative leg pain and may be linked to failed surgery cases.

The observations in human samples prompted us to gain insight into the potential of TNF- $\alpha$ inhibitors to induce analgesic effects, and the mechanisms of action leading to these effects. To this end, we administered several drugs to the sciatic nerve $\mathrm{CCl}$ rat model and analyzed their effects on pain behavior, and TNF- $\alpha$ and TNFR expression in dorsal horn (DH) and dorsal root ganglion (DRG) samples. Chapter 7 describes a positive correlation between hypersensitivity behavior and TNF- $\alpha$ expression in the $\mathrm{DH}$ of $\mathrm{CCl}$ rats. A poor behavioral recovery also coincided with an increased TNFR1/TNFR2 expression ratio. Thalidomide treated $\mathrm{CCl}$ animals showed a significantly faster recovery from pain behavior and an attenuated TNF- $\alpha$ level and TNFR1/TNFR2 ratio. Because these effects were unmet by treatments with NO-711, IGF-1 or 


\section{Chapter 9}

ZVAD, we performed a follow-up study with TNF- $\alpha$ inhibitors that had different affinities for its membranous and soluble form. In chapter $\mathbf{8}$ we describe results obtained from $\mathrm{CCl}$ animals that had been treated with etanercept or infliximab. Though both drugs were able to diminished pain behavior and to reduce TNFR expression in DH and DRG, these effects were strongest in infliximab treated animals. This suggests that pain management can be achieved most effectively by inhibition of membranous TNF- $\alpha$.

Altogether, these findings provide new insights into the physiopathological mechanisms of inflammation in chronic neuropathic pain. We suggest that an unbalance between TNFR1 and TNFR2 is associated with the degree of pain experienced by patients. These findings stress the potential of anti-TNF- $\alpha$ treatment in a subset of LDH patients. In addition, they hint towards the possibility to use TNF- $\alpha$ or its receptors to predict the outcome of LDH surgery. They therefore suggest that the selection criteria for discectomy may be improved. Future studies are required to further identify if these inflammatory profiles are expressed individually in patients, and to unravel the factors that generate these differences. 
Acknowledgements 


\section{Acknowledgements}

"Only one excess is advisable in the world: the excess of gratitude"

- Jean de la Bruyère

It is a real pleasure to thank all the people that made the completion of this thesis possible. First and foremost, I would like to express my gratitude to my team of supervisors and co-supervisors, without whose support and encouragement I would not have been able to reach this objective.

Prof. Visser-Vandewalle, dear Veerle (I finally dared to call you by your first name... or at least to write it down), I would like to thank you for your persistence, understanding and kindness during this period of my academic formation. From the very first meeting that we had, you showed me your resolute commitment to this project. I will always be grateful for your guidance and support during these years. And as if that were not enough, you were always attentive to my personal and professional ambitions. You have helped me so much in order to achieve these goals that it is difficult to overstate my gratitude for you. I am looking forward to continue working with you in the future.

Dr. Hoogland, dear Govert, I will always be indebted to your unfailing and generous support as my co-supervisor. Your constant guidance and dedication to this project stimulated me to work harder in every aspect of our research. Thank you for your patience (especially at the beginning) while you teached me all the ins and outs of molecular techniques... coming from the clinic, it was not an easy task. I would also like to thank you for being a genuine daily supervisor, you always made me feel comfortable to knock on your door at any time in order to discuss literature or plan new experiments. I consider myself immensely fortunate to have worked under your supervision and learn from your resolute dedication towards excellence. I am confident that your critical attitude about science will remain in my way to do research in the future.

Prof. Steinbusch, dear Harry, I would like to thank you for giving me the opportunity to come to this department to perform this project. From the very 
beginning until the end, you were always mindful of my professional and personal development. I will always be grateful for your unconditional support during my time as a PhD student in Maastricht, and for your interest on my future career in science.

Dr. Daemen, dear Marc, thank you for trusting me with this project and for allowing me to incorporate and develop my own ideas into it. Your enthusiasm on the surgical field and your feedback were always greatly received. I will be forever grateful for the confidence invested in me to continue your work.

I offer my sincerest gratitude to the members of the assessment committee and corona for your time and for the critical analysis on my work. I would like to extend this acknowledgement to Prof. Sommer for her support and hospitality during my time in Würzburg. Thank you for the opportunity to visit your lab and learn from you and the members of your team. I enjoyed my time over there working on such a great scientific atmosphere.

A mis mentores en México; Dr. Jiménez, Dr. Carrillo, Dr. Velasco y todo el equipo del Hospital General de México, siempre será un orgullo haber formado parte de su equipo y de su trabajo. Es un honor y un gusto recordar que fueron ustedes quienes me introdujeron al mundo de las neurociencias. Por esto y más, les estaré agradecido por siempre.

I wish to thank all the people from the departments of Neuroscience and Neurosurgery who have helped me in so many ways during this time. To all the co-authors on this thesis; thank you for your invaluable comments and for helping me to improve this project. To the technical team; Marjan, Denise and Helen, thank you for support and help during my experiments, and for your patience with my ongoing learning curve. To the secretary service team; Lisa, Marie-José, Nicole, Anouk and Marie-Thérèse, thank you very much for all your help and for making our life easier. To my colleagues and friends; Fabien, Sonny, Ali, Alejandro, Ehsan, Marlien, Nienke, Mark, Anthony, Youssef, Thibaut, Bart, Rinske, Jo, Frank, Ivona, Jochen, Maria, Dagmar, Sarah, Gerard, 
Chiara, Caroline, Sven, Eva B, Eva vD, Marijke, Tim, Nick, Shahed, Rianne, Kathleen, Jana, Julie, Pierre, Natalia, and many others, thank you for mantaining such an enjoyable scientific environment and for all the fun times inside and outside the lab. To all the students that directly contributed to this thesis; Miguel, John, Caroline, Emiel and Mathias, thank you for your motivation, commitment and interest in our work. To all the physicians and residents from neurosurgery, thank you for your support and cooperation in Heerlen and the aZM during my time in the ORs, and for your interest and critical analysis on my research. To the people from the MAASTRO Clinic; thank you for your friendship and for all the great moments with your group. To all my roommates; Evi, Evelien, Marisela, Annerieke, Michelle and Kimberly, I am more than indebted with each and every one of you. Thank you for all your help and advises in every topic of science and life. You really made the room feel like a second home... maybe a little bit less tidy. And, last but not least, Leonidas; Professor, what can I say, one simply could not wish for a better partner in crime. You were my first friend in this town and you helped me to mantain the perfect balance between work and fun during all these years. I will miss our discussions about life during dinner at the hospital or a traditional protocol ceremony. I wish you all the best in your new job, with the last sprint of your thesis and in your future career. Efharisto poli!

To my paranymphen, Evi and Emmanuel, thank you again for helping me during this process. Evi; geachte Evi (I think I have never learned the right/nonformal way to write it down), I really appreciate everything you have done for me during my stay in Maastricht. Thank you for being always available, from simple language aid, to serious help with my experiments or data analysis. Even now, with the closing of your own project you accepted to assist me on this one. For all your support and your friendship...Heel erg bedankt! Emmanuel; dicen que las palabras sobran, pero en este caso creo que hacen falta para agradecerte lo suficiente. Aunque haría falta otro libro completo para escribir tantas aventuras, intentaré ser breve con este agradecimiento. Siempre dijimos que disfrutar Maastricht dependía de la gente con la que la compartías, pues puedo asegurarte que fue una experiencia excelente. Gracias por ser un excelente amigo y por acompañarme en este proceso una vez más. Y mientras llega la siguiente... Youf! 
A mis hermanos; Fer y Vicky, creo que jamás encontraré la forma de agradecer su apoyo, comprensión y confianza. Al igual que todo lo que tengo en esta vida, quiero compartir este triunfo por siempre con ustedes. Les pido una disculpa por todas mis ausencias. Sepan que siempre estuvieron conmigo y que todo esto fue para hacerlos sentirse orgullosos de esta persona que tanto los ama.

A mis padres; Ma y $\mathrm{Pa}$, gracias por todo el cariño que siempre he recibido de ustedes. Desde el principio me han otorgado mucho más de lo que he necesitado. Gracias a su apoyo constante es que he podido culminar otra etapa en mi educación, esta será por siempre la mejor de todas las herencias. Sólo espero que comprendan que mis ideales, esfuerzos y logros han sido también suyos e inspirados en ustedes. Con amor, admiración y respeto infinito... muchas gracias.

Enfin, Lauriane; ma chère PPLP, ces remerciements ne seraient pas complets sans te mentionner. Rien de tout cela n'aurait été possible sans ton soutien quotidien au cours de ces longues années d'études et sans lesquels je n'en serai pas là aujourd'hui. Merci pour ta patience pendant cette période, en particulier dans les jours difficiles de ce projet. C'est grâce à ton amour et ton soutien indéfectible que nous pouvons maintenant partager ce moment. Parce que comme toujours, cette victoire appartient à nous deux. Gracias... 



\section{Curriculum Vitae}

Pablo Andrade was born on April $10^{\text {th }} 1983$ in Tlalnepantla de Baz, Mexico. He grew up in Naucalpan de Juárez where he graduated from high school at 'Centro Escolar del Tepeyac' in 2001. The following year, he started his medical studies at Anáhuac University, School of Medicine in Mexico City (2002-2008). During this period, he performed his medical and surgical internships at the Jackson Memorial Hospital, University of Miami (USA). After receiving his medical doctor degree, he worked for one year at the Laboratory of Experimental Neurology and the Functional Neurosurgery Clinic at Mexico General Hospital focusing on deep brain stimulation for psychiatric disorders. Later that year, he received the 'New Investigator Competition' award from the International Neuromodulation Society (INS) for his work on an experimental model of compulsive behavior. In August 2009, he started as a PhD student at the departments of Neuroscience and Neurosurgery at Maastricht University Medical Center (Netherlands) under the supervision of Prof. Dr. Veerle VisserVandewalle, Prof. Dr. Harry Steinbusch and Dr. Govert Hoogland. During this 3year period, he investigated the role of TNF-alpha and its receptors in the pathogenesis of chronic pain. In early 2012, he was awarded an 'InEurope Short-Stay' Fellowship from the International Brain Organization (IBRO), which gave him the opportunity to visit the Pain Research Laboratory at the University of Würzburg (Germany) where he worked on different aspects related to neuroinflammation. The results of his $\mathrm{PhD}$ project are described in this thesis. 

List of Publications 


\section{Journal Articles}

1. Andrade $\mathbf{P}$, Carrillo-Ruiz JD, Jiménez F. A systematic review of the efficacy of globus pallidus stimulation in the treatment of Parkinson's disease. J Clin Neurosci 2009; 16: 877-881.

2. Andrade $\mathbf{P}$, Fernández-Guasti $A$, Carrillo-Ruiz JD, Ulloa RE, Ramírez $Y$, Reyes $R$, Jiménez $F$. Effects of bilateral lesions in thalamic reticular nucleus and orbitofrontal cortex in a T-maze perseverative model produced by 8-OH-DPAT in rats. Behav Brain Res 2009; 203: 108-112.

3. Carrillo-Ruiz JD*, Andrade P*, Silva F, Vargas G, Maciel-Navarro MM, Jiménez-Botello LC. Olfactory bulb implantation and methylprednisolone administration in the treatment of spinal cord injury in rats. Neurosci Lett 2009; 462: 39-44. ( ${ }^{*}$ Contributed equally to this work)

4. Andrade $\mathbf{P}$, Carrillo-Ruiz JD, Ramírez $Y$, Jiménez F. Effects of thalamic reticular nucleus electrical stimulation in rats in a T-maze perseverative behavior model induced by 8-OH-DPAT. Neuromodulation 2010; 13 : 29.

5. Carrillo-Ruiz JD, Andrade P, Godínez-Cubillos N, Montes-Castillo ML, Jiménez F, Velasco AL, Castro G, Velasco F. Coupled obturator neurotomies and lidocaine intrathecal infusion to treat bilateral adductors spasticity and drug-refractory pain. J Neurosurg 2010; 113: 528-531.

6. Andrade P, Noblesse LH, Temel Y, Ackermans L, Lim LW, Steinbusch $\mathrm{HW}$, Visser-Vandewalle V. Neurostimulatory and ablative treatment options in major depressive disorder. A systematic review. Acta Neurochirur (Wien) 2010; 152: 565-577.

7. Andrade $\mathbf{P}$, Visser-Vandewalle V, Hoffmann C, Steinbusch HW, Daemen MA, Hoogland G. Role of TNF-alpha during central sensitization in preclinical studies. Neurol Sci 2011; 32: 757-771.

8. Andrade $\mathbf{P}$, Visser-Vandewalle $V$, Philippens $M$, Daemen MA, Steinbusch HW, Buurman WA, Hoogland G. TNF-alpha levels correlate with postoperative pain severity in lumbar disc hernia patients: Opposite clinical effects between TNF receptor 1 and 2. Pain 2011; 152: 2645-2652.

9. Andrade $\mathbf{P}$, Visser-Vandewalle V, Del Rosario JS, Daemen MA, Buurman WA, Steinbusch HW, Hoogland G. The thalidomide analgesic effect is associated with differential TNF- $\alpha$ receptor expression in the 
dorsal horn of the spinal cord as studied in a rat model of neuropathic pain. Brain Res 2012; 1450: 24-32.

10. Jiménez $F$, Soto JE, Velasco $F$, Andrade $P$, Bustamante JJ, Gómez $P$, Ramírez Y, Carrillo-Ruiz JD. Bilateral cingulotomy and anterior capsulotomy applied to patients with aggressiveness. Stereotact Funct Neurosurg 2012; 90: 151-160.

11. Andrade P, Hoogland G, Daemen MA, Garcia MA, Steinbusch HW, Visser-Vandewalle V. Elevated levels of IL-1beta and IL-6 in patients with sciatic pain related to lumbar herniated disc. Revision submitted.

\section{Abstracts}

1. Poster Presentation. "TNF-alpha levels correlate with postoperative pain severity in lumbar disc hernia patients" 9th Dutch Endo-NeuroPsycho Meeting. Lunteren, The Netherlands (2011).

2. Oral Presentation. "Deep brain stimulation in the Inferior Thalamic Peduncle (ITP) as Treatment of Obsessive Compulsive Disorder" 10th International Neuromodulation Society World Congress. London, United Kingdom (2011).

3. Poster Presentation. "Neuropathic pain mechanisms produced by TNF$\alpha$ in disc hernia patients" XL Annual Meeting Society for Neuroscience. San Diego, USA (2010).

4. Poster Presentation. "Clinical evaluation of molecular mechanisms involved in degenerative disc related pain" FENS-IBRO European Pain School. Siena, Italy (2010).

5. Oral Presentation. "Beneficial Results from Lidocaine Infusion Pump Implantation and Obturators Neurotomies for Intractable Pain and Adductors Spasticity in One Patient after 36 Months' Follow-Up" 9th International Neuromodulation Society World Congress. Seoul, South Korea (2009).

6. Poster Presentation. "Effects of thalamic reticular nucleus electrical stimulation in rats in a T-maze perseverative behavior model". 9th International Neuromodulation Society World Congress. Seoul, South Korea (2009).

7. Poster Presentation. "Efficacy of globus pallidus internus stimulation in treatment of Parkinson's disease: a meta-analysis". XVIII Congress of the European Society for Stereotactic and Functional Neurosurgery. Rimini, Italy (2008). 
8. Oral Presentation. "Effect of Stereotactic Lesion of Reticular Thalamic Nucleus in a T-Maze Perseverative model in Wistar rats". XVIII Congress of the European Society for Stereotactic and Functional Neurosurgery. Rimini, Italy (2008).

9. Poster Presentation. "Correlation Study Between Motor Outcome and Stimulation Parameters of Globus Pallidus in Patients with Parkinson's Disease". 8th International Neuromodulation Society World Congress. 11th North American Neuromodulation Society Annual Meeting. Acapulco, Mexico (2007). 


$$
\begin{array}{r}
\text { List of } \\
\text { Abbreviations }
\end{array}
$$


$\mathrm{AF}=$ annulus fibrosus; $\mathrm{BDNF}=$ Brain-derived neurotrophic factor; $\mathrm{C} 1 \mathrm{q}=$ Complement component-1, q-subcomponent; C3= Complement component-3; $\mathrm{CANP}=$ Non-inflammatory chronic axonal neuropathy; $\mathrm{CCl}=$ Chronic constriction injury; $C F S / F M S=$ Chronic fatigue syndrome/ fibromyalgia syndrome; $\quad \mathrm{CGRP}=$ Calcitonin gene-related peptide; $\mathrm{CIDP}=$ Chronic inflammatory demyelinating neuropathy; $\mathrm{CLBP}=$ Chronic low back pain; $\mathrm{CP}=$ Chronic Pain; CRPS = Complex regional pain syndrome; CWP = Chronic widespread pain; $\mathrm{DH}=$ Dorsal horn; $\mathrm{DPPN}=$ Distal painful non-diabetic polyneuropathy; $\mathrm{DRG}=$ Dorsal root ganglion; ELISA $=$ Enzyme-linked immunosorbent assay; ET-1= Endothelin-1; fLDH= First time lumbar disc hernia; $f+r L D H=$ First time and recurrent lumbar disc hernia; GABA= Gammaaminobutyric acid; GCPS = Graded chronic pain scale; GFAP = Glial fibrillary acidic protein; Glu= Glutamate; Gly= Glycine; GM-CSF= Granulocyte macrophage-colony stimulating factor; IFN- $\alpha=$ Interferon-alpha; IL-10= Interleukin-10; IL-12= Interleukin-12; IL-13= Interleukin-13; IL-17= Interleukin17; IL-1R= Interleukin-1 receptor; IL-1ra= Interleukin-1 receptor antagonist; IL$1 ß=$ Interleukin-1 beta; IL-2= Interleukin-2; IL-4= Interleukin-4; IL-6= Interleukin6; IL-6R= Interleukin-6 receptor. IL-8= Interleukin-8; IP-10= Interferon-inducible protein 10; IVD= Intervertebral disc; JOAS= Japanese orthopedic association score; $L D H=$ Lumbar disc hernia; $L F=$ Ligamentum flavum; LSCS= Lumbar spinal canal stenosis; LSS= Lumbar spinal stenosis; $L T P=$ Long-term potentiation; $\mathrm{MAPK}=$ Mitogen-activated protein kinase; $\mathrm{MBL}=$ Monoclonal $\mathrm{B}$-cell lymphocytosis; MCP-1= Monocyte chemotactic protein-1; MIG= monokine induced by gamma interferon; MIP-1 $1=$ Macrophage inflammatory protein- $1 ß$; MIP-1 $\alpha=$ Macrophage inflammatory protein $-1 \alpha ; \quad M P Q=$ McGill Pain Questionnaire; NDS= Neurological disability score; NO= Nitric oxide; NP= Nucleus pulposus/ Neuropathic pain; $N P Q=$ Neuropathic pain questionnaire; NPS $=$ Neuropathic pain scale; NPSD $=$ German version of the neuropathic pain scale; NPSI= Neuropathic pain symptom inventory; NPY= Neuropeptide Y; NRS= Numeric rating scale; NSS= Neuropathy symptom score; $\mathrm{PBMC}=$ Peripheral blood mononuclear cells; $\mathrm{PGE} 2=$ Prostaglandin E2; $\mathrm{PKA}=$ Protein kinase $\mathrm{A} . \mathrm{PKC}=$ Protein kinase $\mathrm{C} ; \mathrm{POEMS}=$ Polyneuropathy, organomegaly, endocrinopathy, monoclonal gammopathy and skin changes; PTN= Posttraumatic neuralgia; $C S F=$ Cerebrospinal fluid; $q P C R=$ Quantitative polymerase chain reaction; QST= Quantitative sensory testing; RANTES= Regulated upon activation, normal $\mathrm{T}$-cell expressed and secreted; $\mathrm{rLDH}=$ Recurrent lumbar disc hernia; $\mathrm{RMQ}=$ Roland and Morris questionnaire; $\mathrm{SCl}=$ Spinal cord injury; sICAM-1 = Soluble intercellular adhesion molecule-1; $\mathrm{slL}-6 \mathrm{R}=$ Soluble interleukin-6 receptor; SP= Scoliosis patients; sTNFR1= Soluble tumor necrosis 
factor (TNF) receptor-1; sTNFR2= Soluble tumor necrosis factor (TNF) receptor-2; Sub $\mathrm{P}=$ Substance $\mathrm{P}$; TGF- $\mathrm{B} 1=$ Transforming growth factor-1; TNF$\alpha=$ Tumor necrosis factor-alpha; Toll-R= Toll-like receptor; VANP $=$ Vasculitic neuropathy; VAS $=$ Visual analogue scale; VPT= Vibration perception threshold. 



$$
\text { Tables }
$$




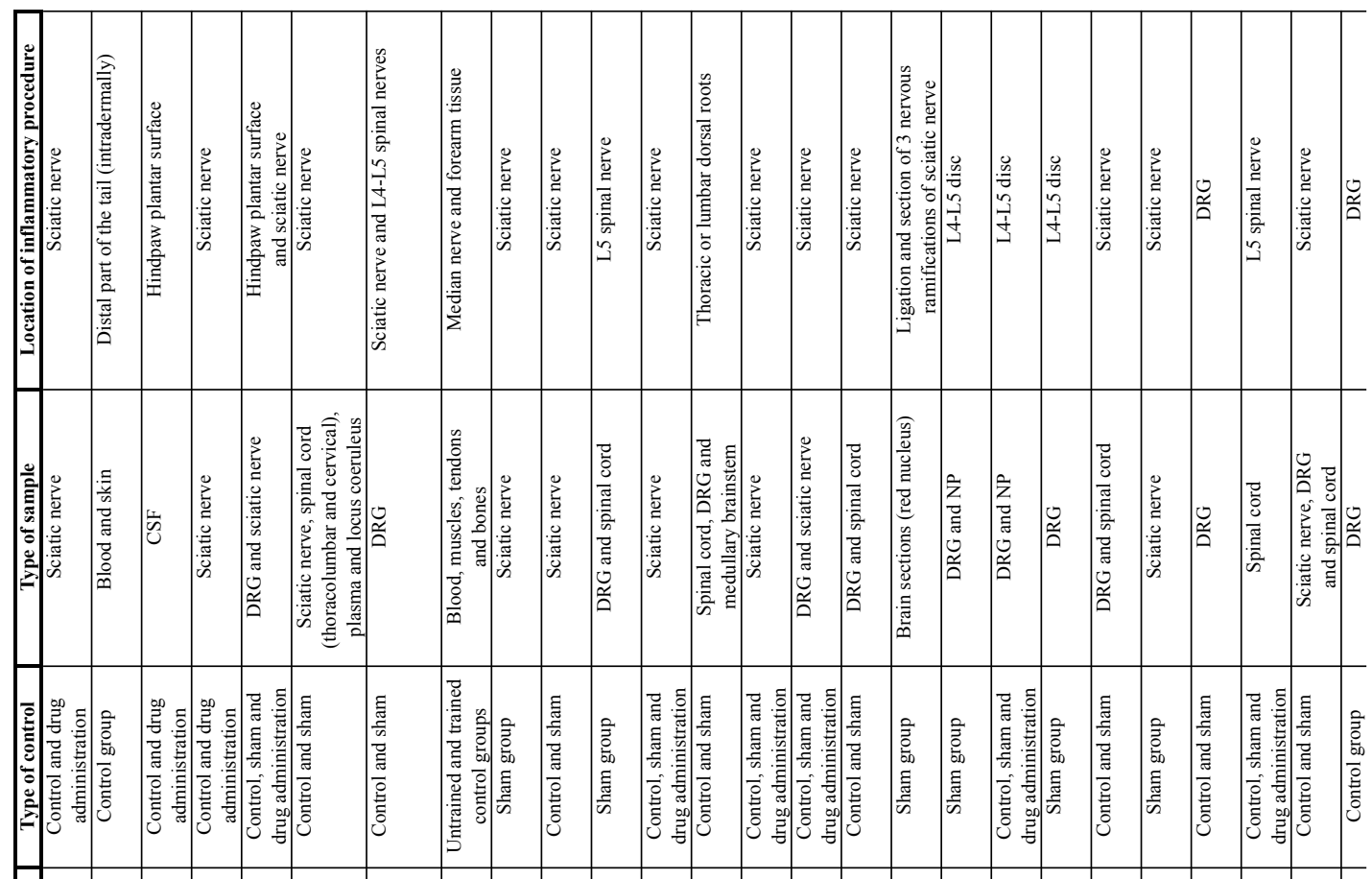

言

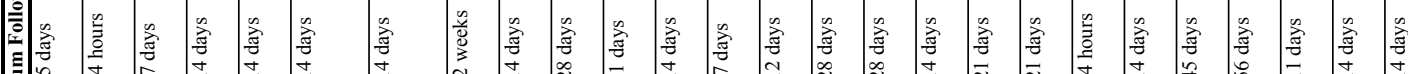

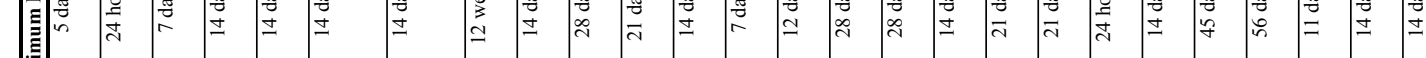

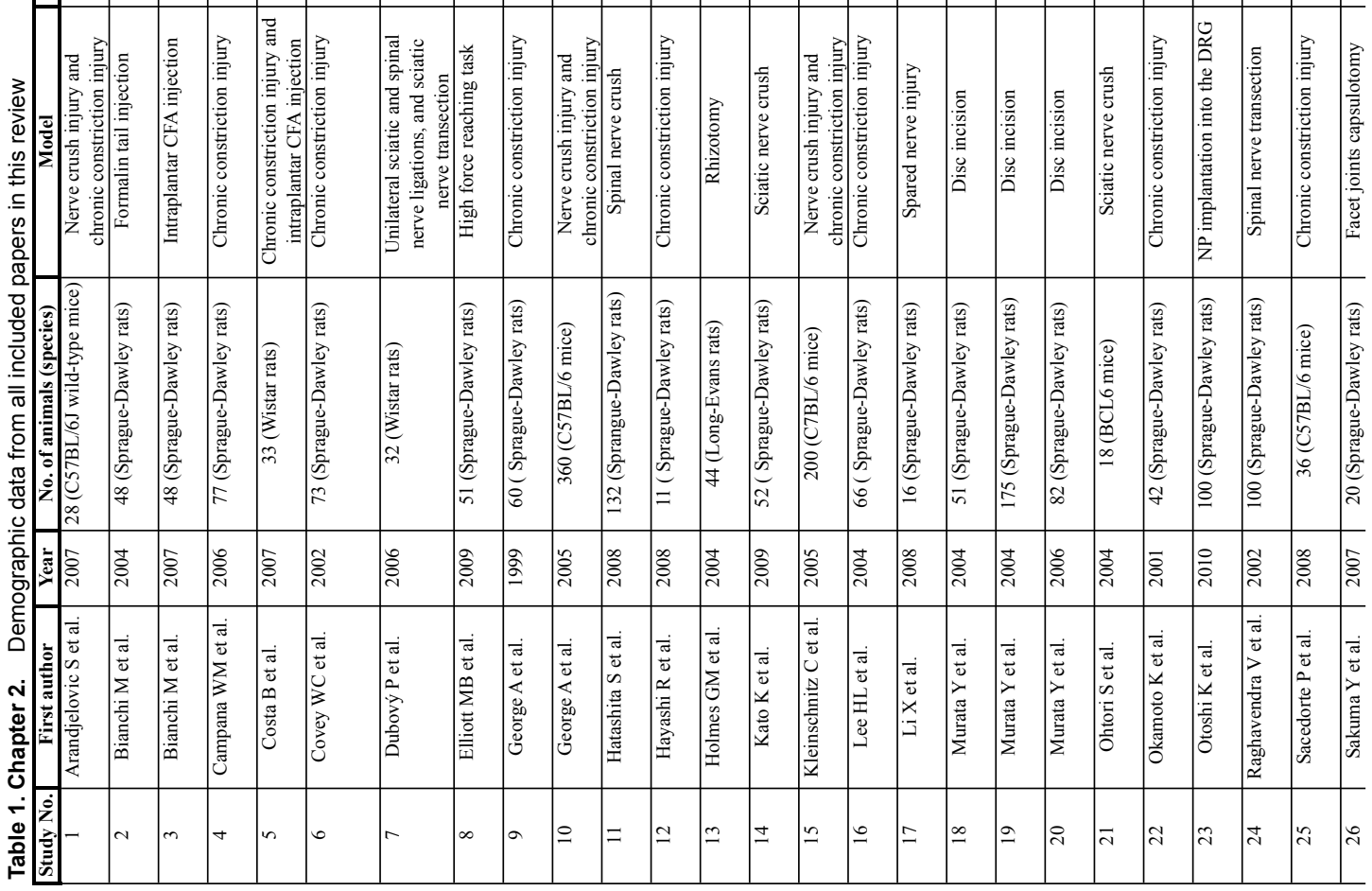




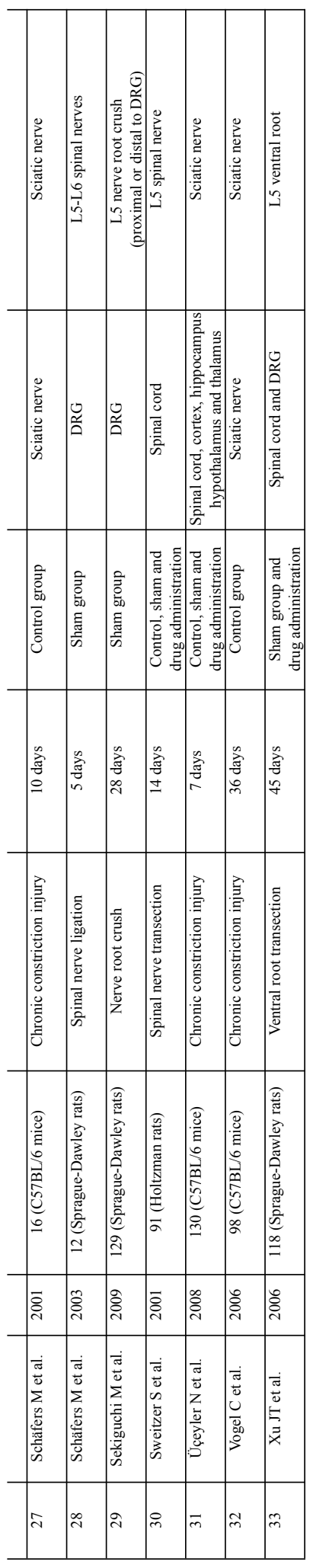




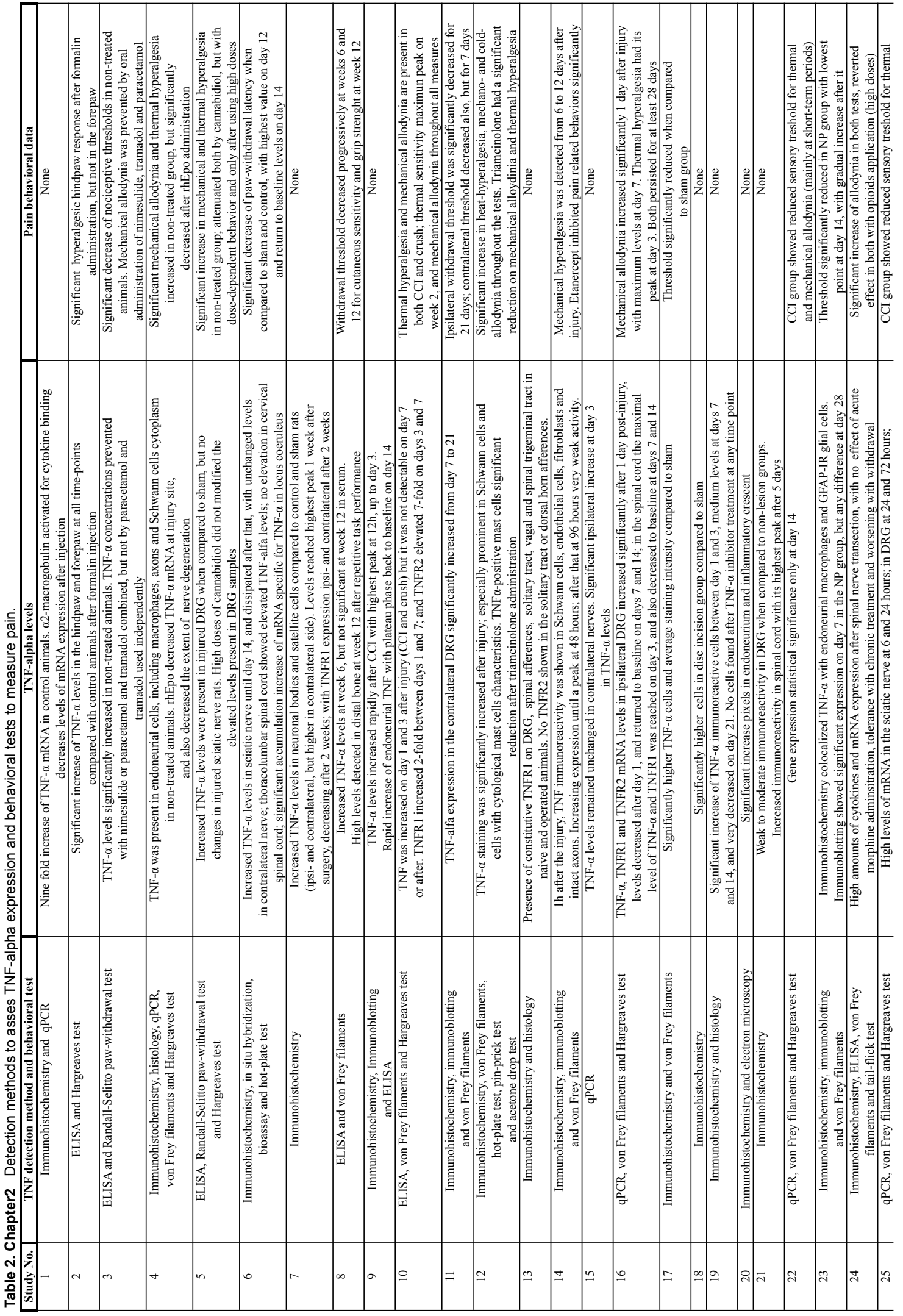




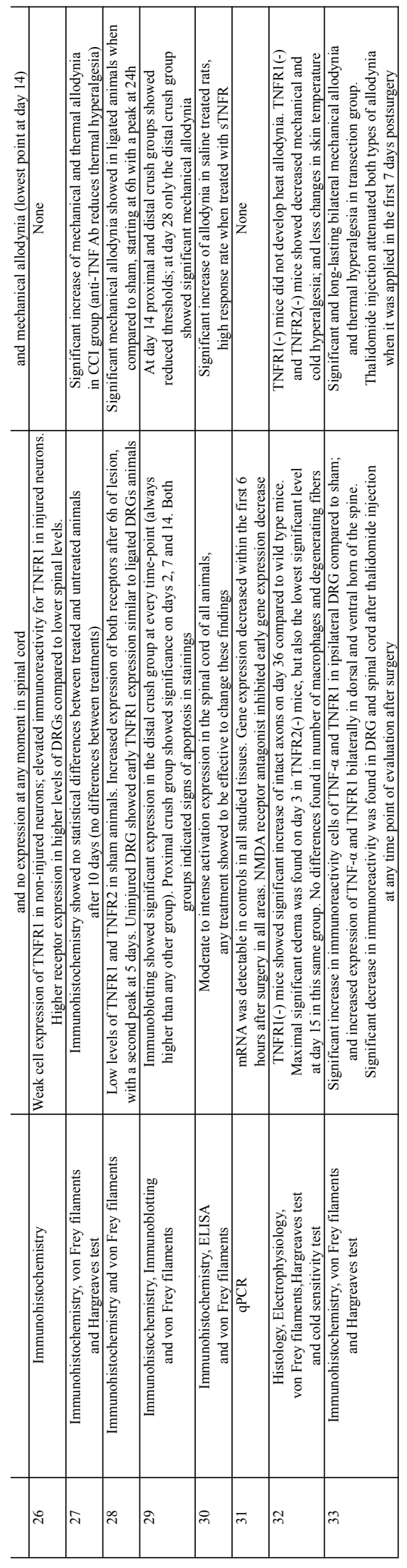




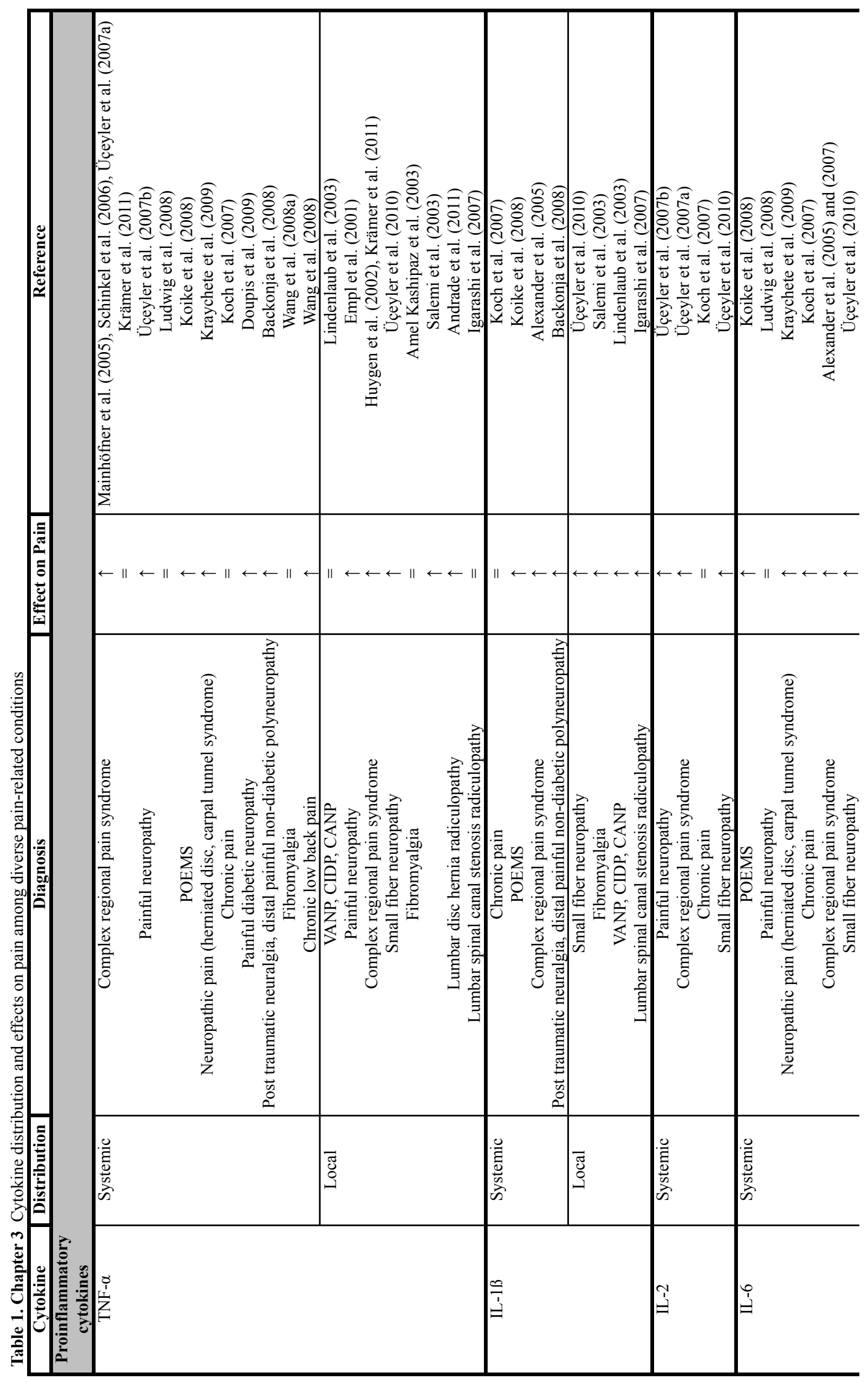




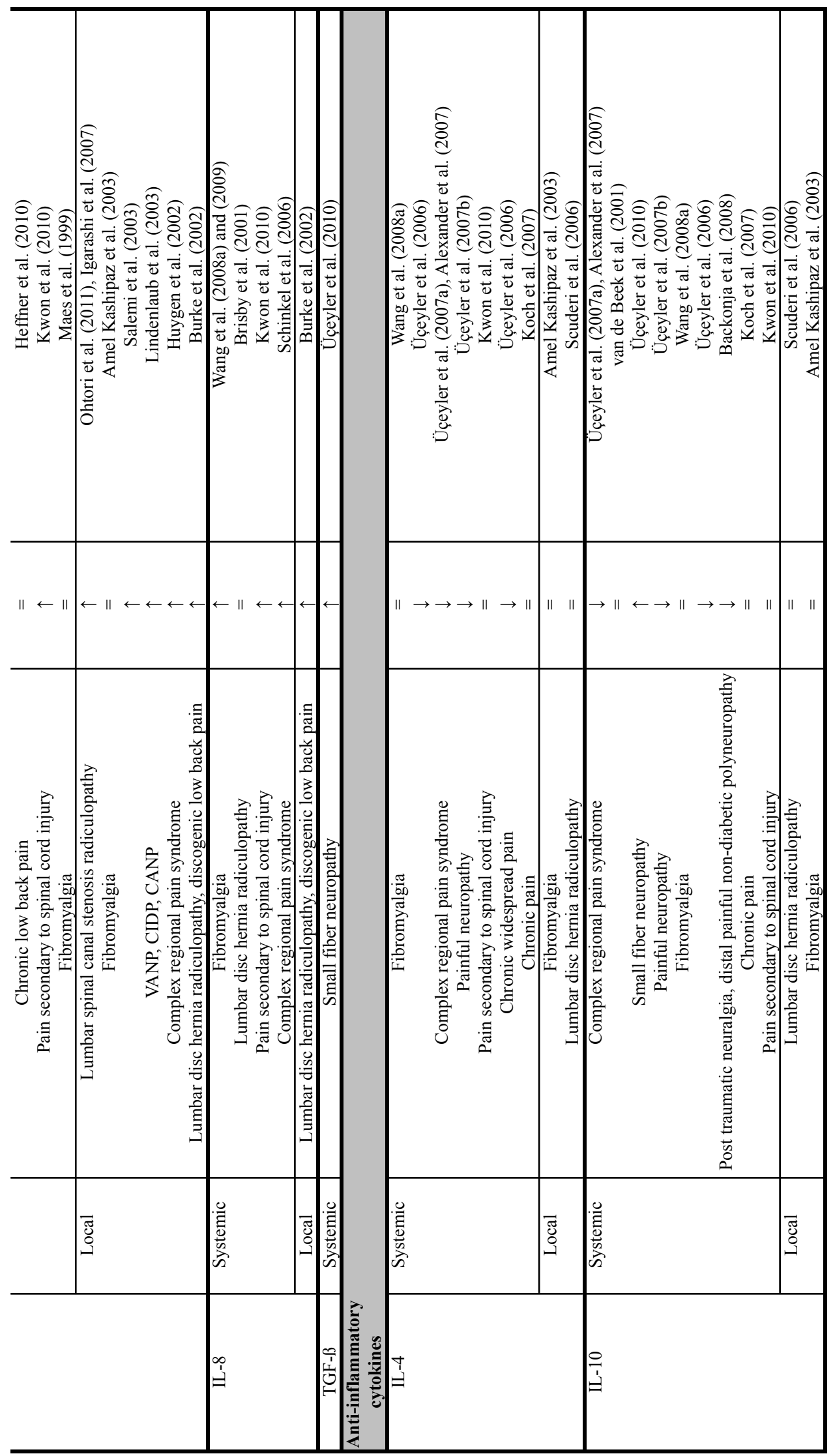




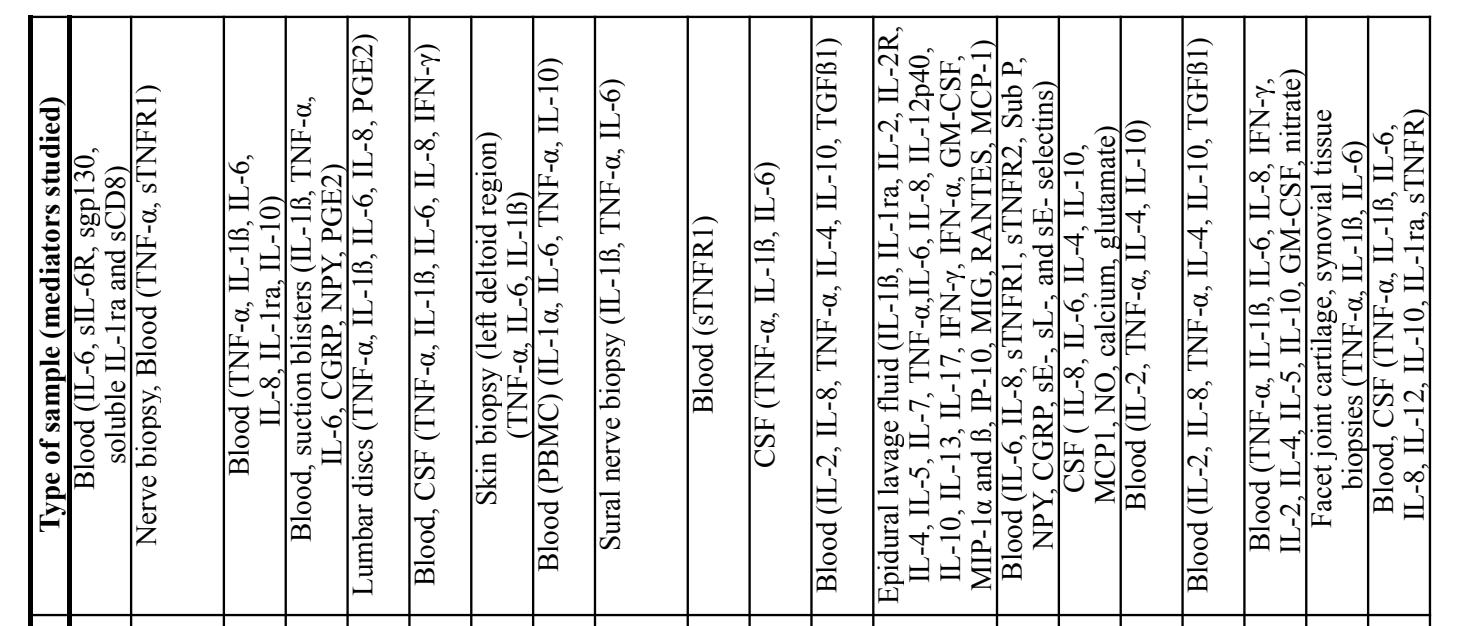

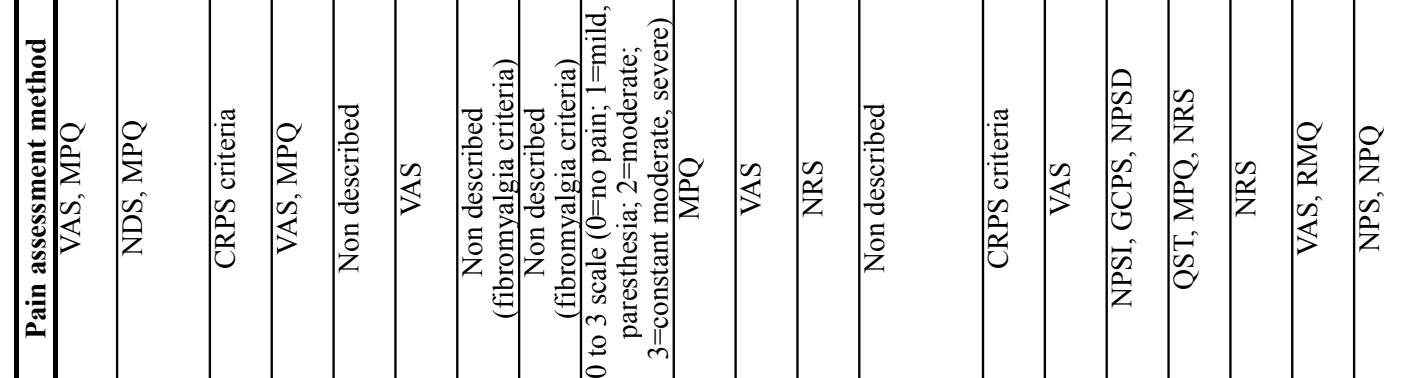

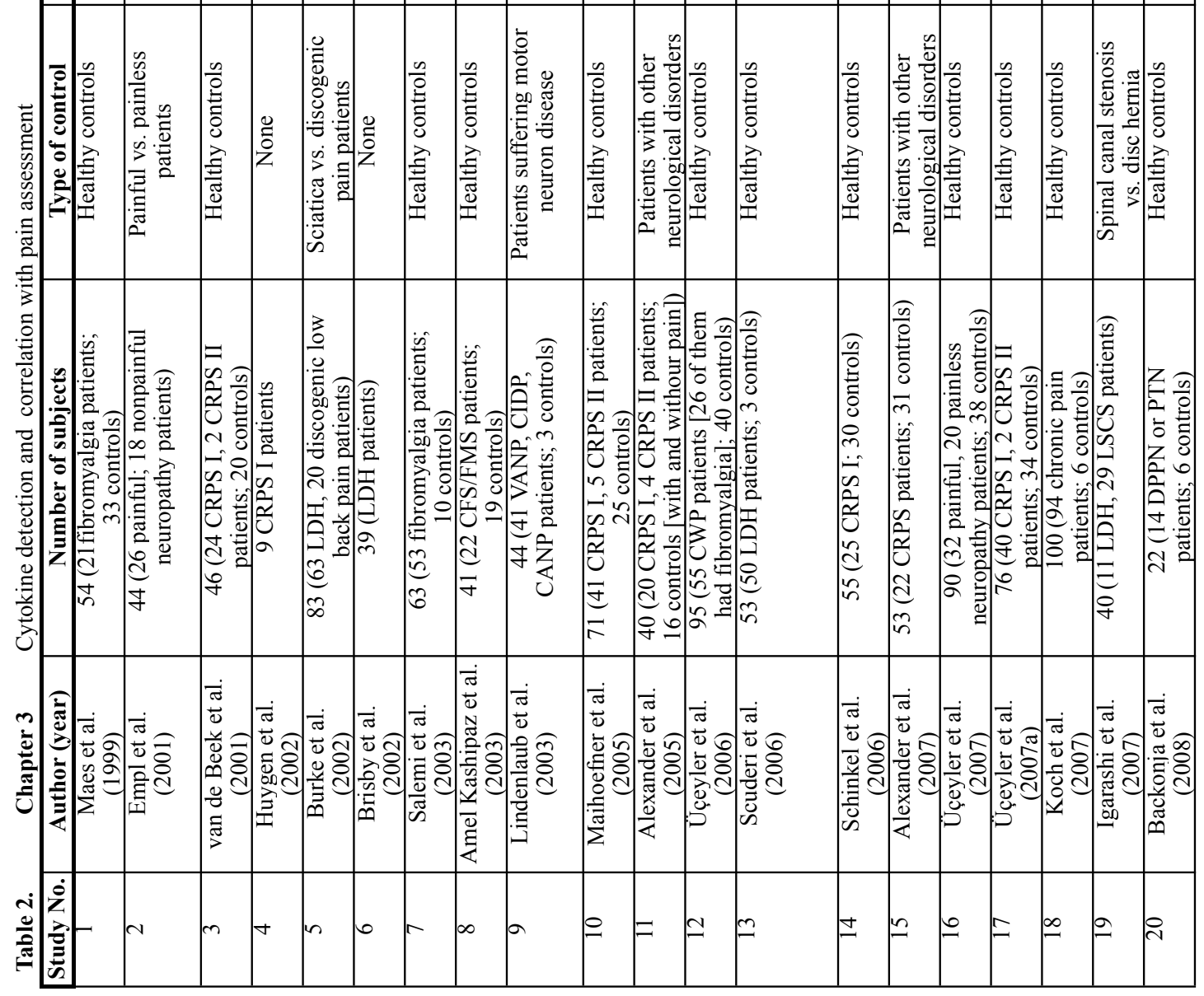




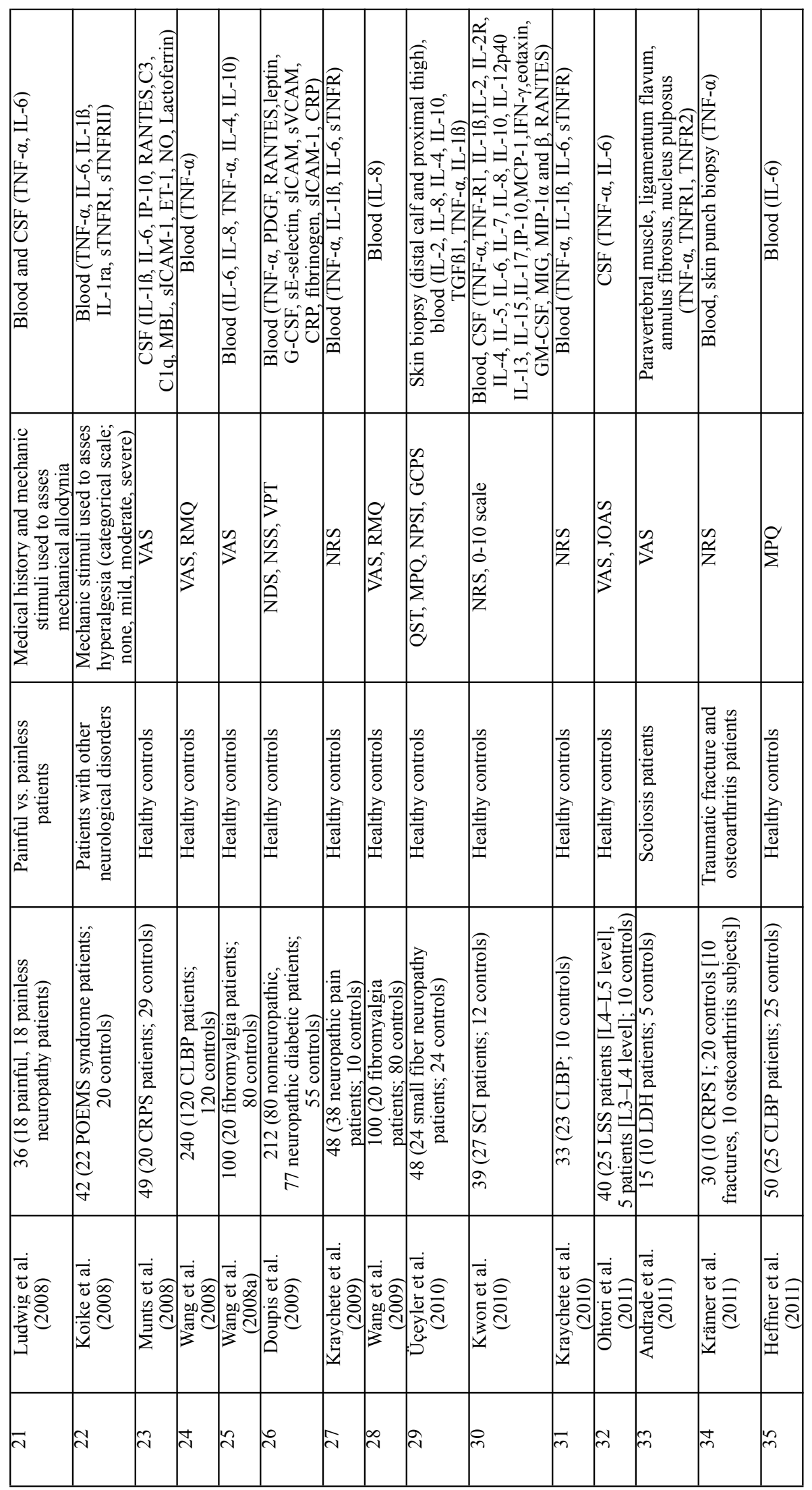




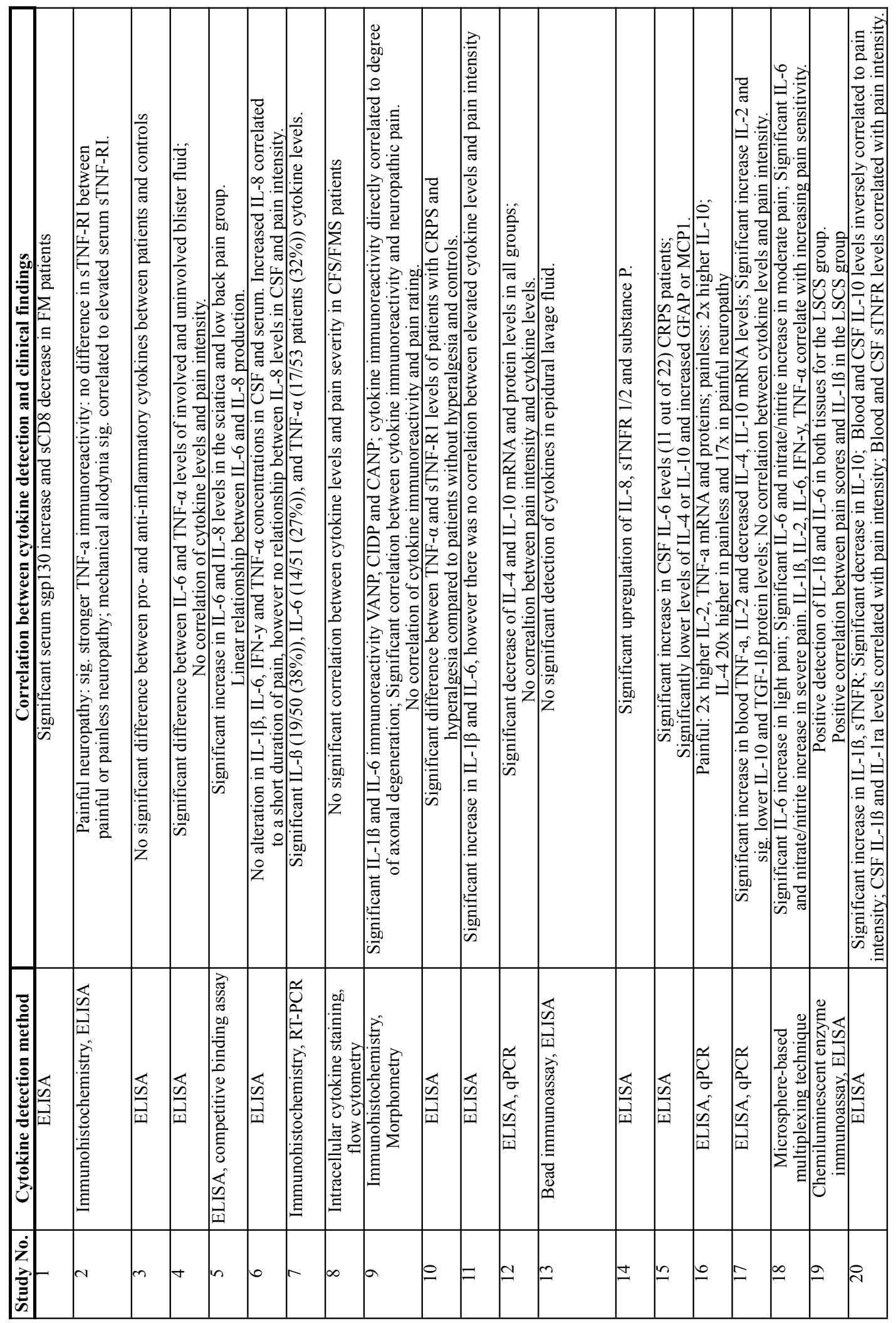




\begin{tabular}{|c|c|c|c|c|c|c|c|c|c|c|c|c|c|c|}
\hline 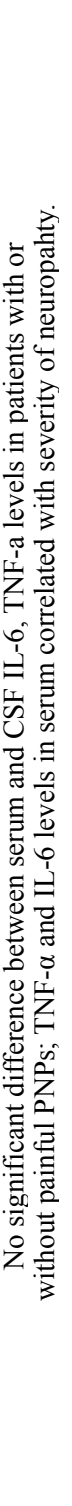 & 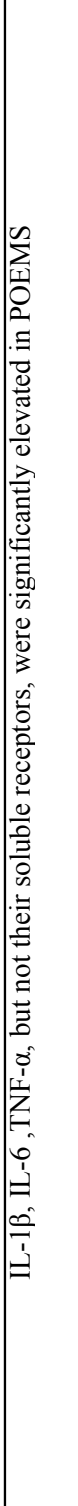 & 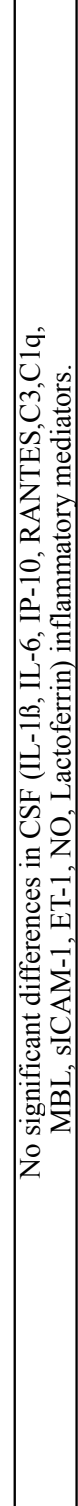 & 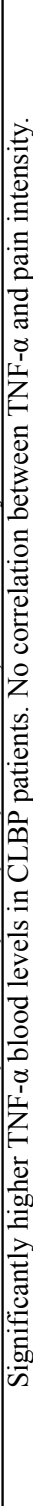 & 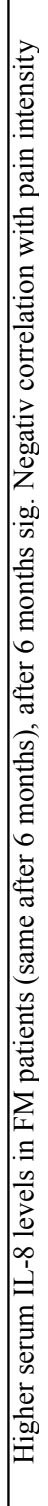 & 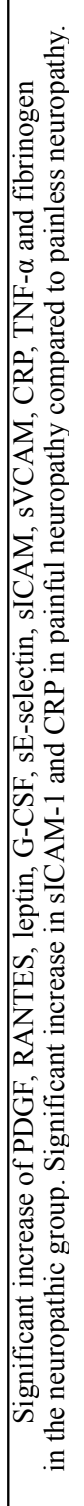 & 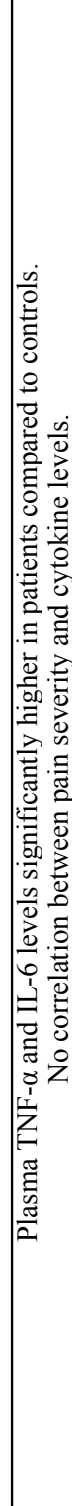 & 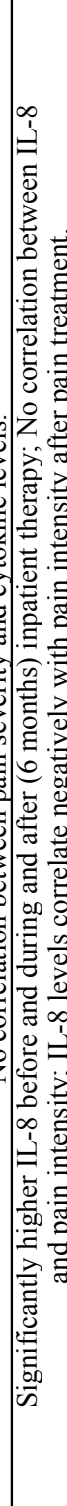 & 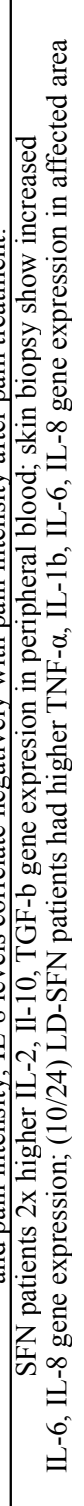 & 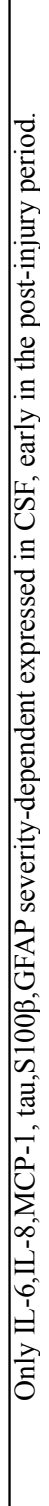 & 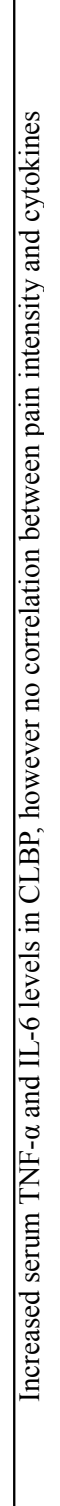 & 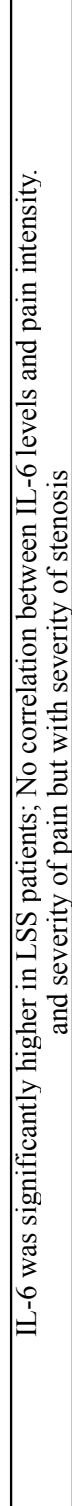 & 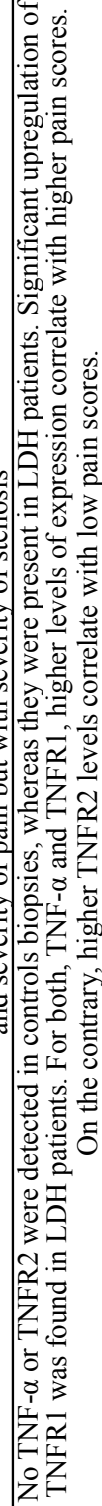 & 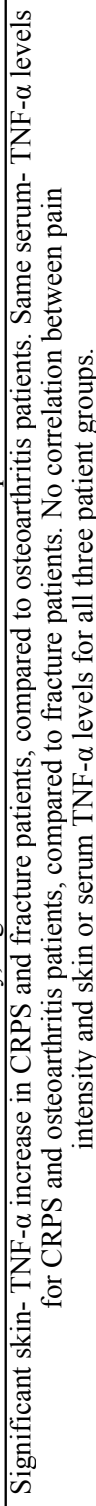 & 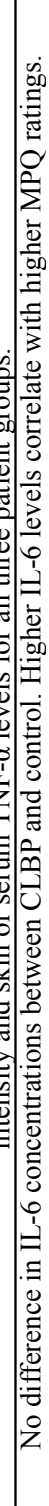 \\
\hline 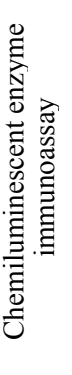 & 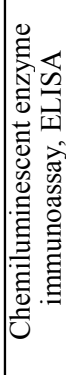 & 兏 & 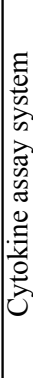 & 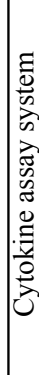 & 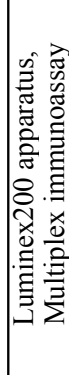 & 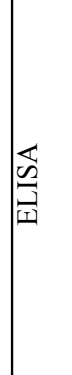 & 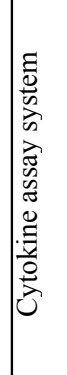 & 促 & 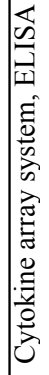 & 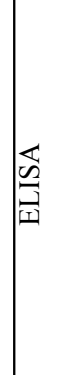 & 虑 & 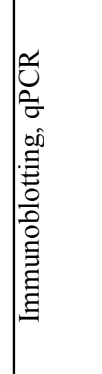 & 岃 & 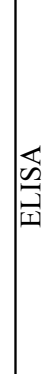 \\
\hline$\vec{\sim}$ & $\sqrt{2}$ & $\therefore$ & 汶 & $\sim$ & $\sim$ & $\sim$ & $\sim$ & $\vec{\imath}$ & $m$ & $m$ & m & $\hat{m}$ & m & $m$ \\
\hline
\end{tabular}




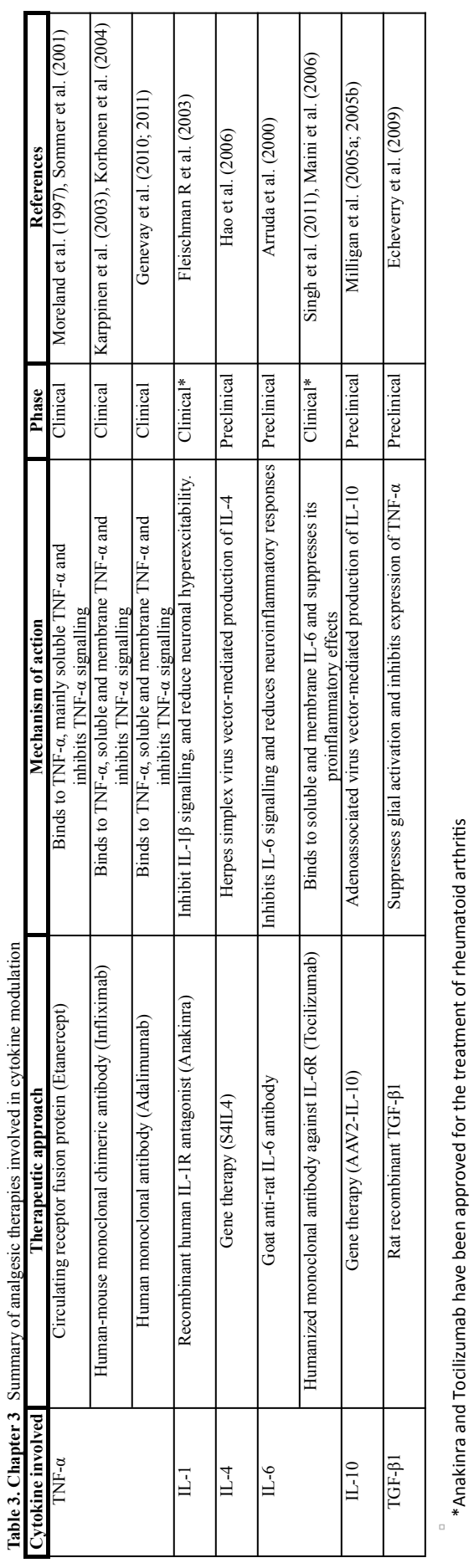




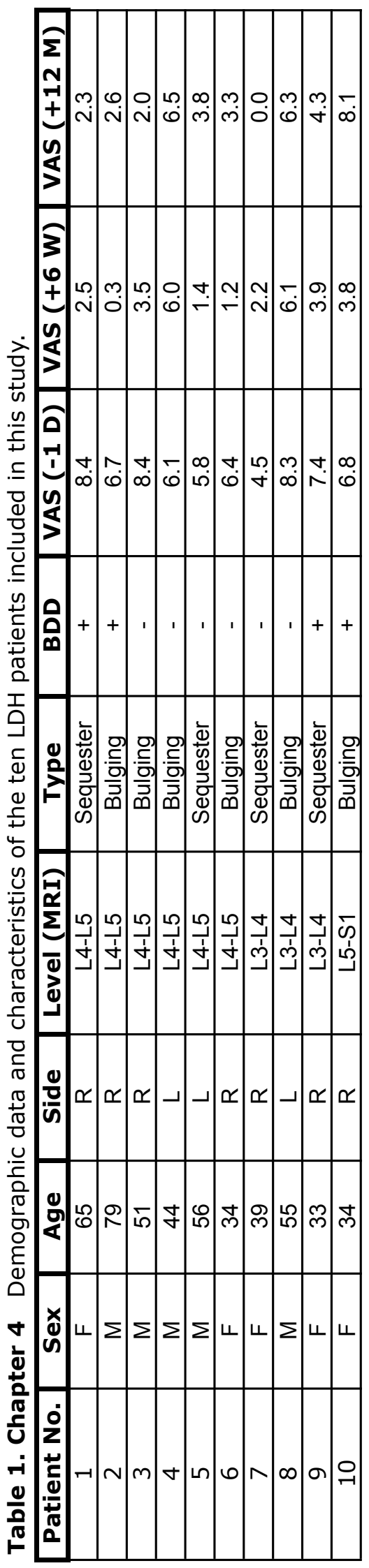




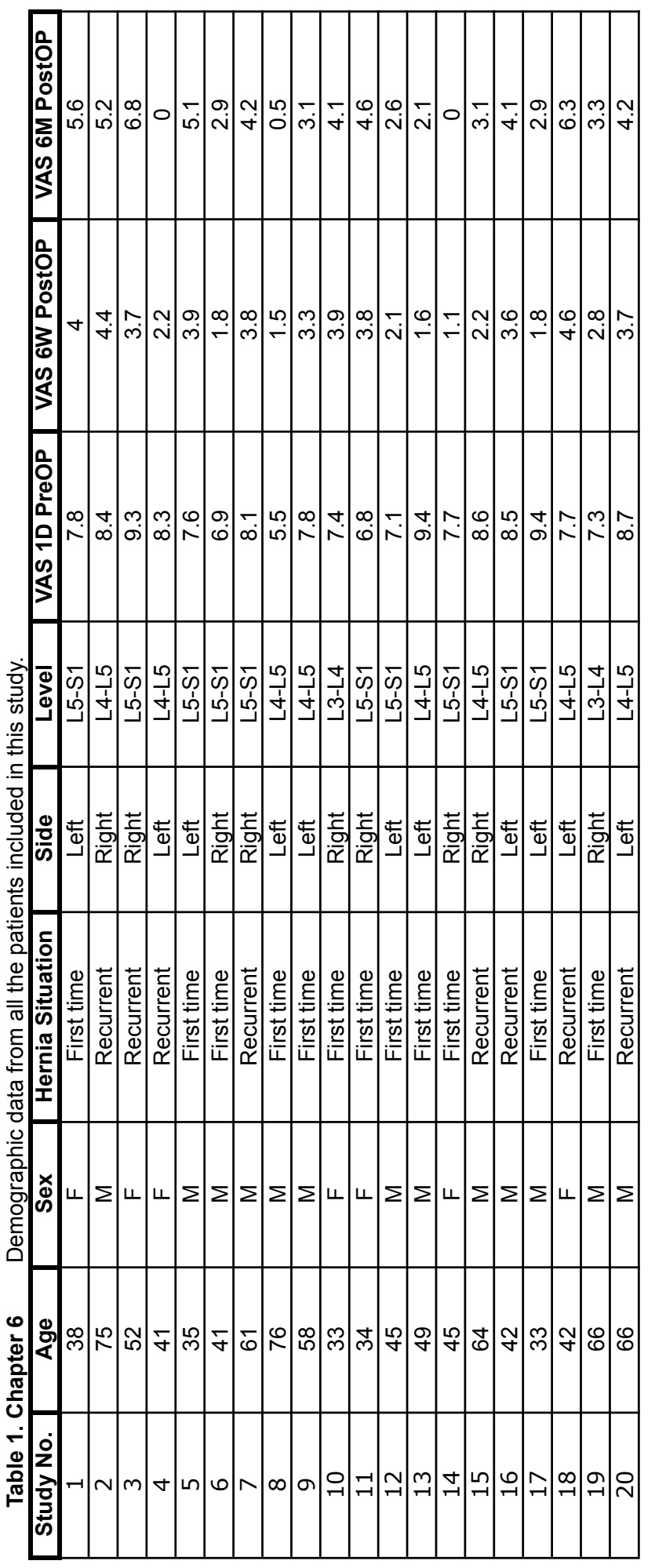



